\title{
REFERENCE MEASUREMENT SYSTEMS IN GENETIC TESTING: DEVELOPMENT OF DNA-BASED REFERENCE MATERIALS
}

PhD thesis

János Márki-Zay MD

\author{
Supervisors: \\ Christoph L. Klein PhD MBA \\ Prof. László Dux MD PhD DSc
}

Reference Materials Unit, Institute for Reference Materials and Measurements European Commission, Joint Research Centre, Geel, Belgium

\author{
and \\ Department of Biochemistry, Faculty of Medicine \\ University of Szeged, Hungary
}


This thesis is dedicated to my wife Mónika 


\section{LIST OF PUBLICATIONS RELATED TO THE THESIS}

I. Klein CL, Márki-Zay J, Corbisier P, Gancberg D, Cooper S, Gemmati D, Halbmayer WM, Kitchen S, Melegh B, Neumaier M, Oldenburg J, Oppliger Leibundgut E, Reitsma PH, Rieger S, Schimmel HG, Spannagl HG, Tordai A, Tosetto A, Visvikis S, Zadro R, Mannhalter C. Reference Materials (RMs) for the analysis of the human factor II (prothrombin) gene G20210A mutation. Clin Chem Lab Med 43:862-68. (2005)

II. Gancberg D, Márki-Zay J, Corbisier P, Klein C, Schimmel H, Emons H. Certification of a Reference Material consisting of purified plasmid DNA containing a fragment from the human prothrombin gene (wildtype) - Certified Reference Material IRMM/IFCC-490. Report EUR 22169 EN (2006)

III. Gancberg D, Márki-Zay J, Corbisier P, Klein C, Schimmel H, Emons H. Certification of a Reference Material consisting of purified plasmid DNA containing a fragment from the human prothrombin gene (G20210A mutant) - Certified Reference Material IRMM/IFCC491. Report EUR 22170 EN (2006)

IV. Gancberg D, Márki-Zay J, Corbisier P, Klein C, Schimmel H, Emons H. Certification of a Reference Material consisting of purified plasmid DNA containing a fragment from the human prothrombin gene (heterozygous G20210 wildtype/G20210A mutant) - Certified Reference Material IRMM/IFCC-492. Report EUR 22167 EN (2006)

V. Gancberg D, Corbisier P, Meeus N, Márki-Zay J, Mannhalter C, Schimmel H. Certification of reference materials for detection of the human prothrombin gene G20210A sequence variant. Clin Chem Lab Med 46:463-9. (2008)

VI. Márki-Zay J, Klein CL, Gancberg D, Schimmel HG, Dux L. European External Quality Control Study on the Competence of Laboratories to Recognize Rare Sequence Variants Resulting in Unusual Genotyping Results. Clin Chem 55:739-47. (2009) 


\section{PRESENTATIONS DIRECTLY RELATED TO THE THESIS}

VII. Márki-Zay J, Klein CL, Corbisier P, Gancberg D, Schimmel H, Mannhalter C. How to prepare stable reference materials for genetic testing. European Human Genetics Conference 2004, 1215. June 2004, Munich, Germany

Abstract: Eur J Hum Genet 12;Suppl 1:235-6[P697] (2004)

VIII. Klein CL, Rieger S, Gancberg D, Márki-Zay J, Corbisier P, Schimmel H, Mannhalter C. Production of Certified Reference Materials (CRMs) for the analysis of the human factor II (prothrombin) gene G20210A mutation. European Human Genetics Conference 2004, 12-15. June 2004, Munich, Germany

Abstract: Eur J Hum Genet 12;Suppl 1:236[P698] (2004)

IX. Barton D, Donohoe EM, Márki-Zay J, Klein CL. Preparation of certified reference materials for hereditary haemochromatosis. European Human Genetics Conference 2004, 12-15. June 2004, Munich, Germany

Abstract: Eur J Hum Genet 12;Suppl 1:257[P803] (2004)

X. Barton DE, Duke S, Donohoe E, Márki-Zay J, Klein CL and the CRMGEN Consortium. The CRMGEN Project: Preparation of Certified Reference Materials for Hereditary Haemochromatosis Testing. $10^{\text {th }}$ Annual Meeting of the Association for Molecular Pathology, 10-13. Nov 2004, Los Angeles, USA

Abstract: J Mol Diagn 6;4:413[G35] (2004)

XI. Gancberg D, Corbisier P, Klein C, Mannhalter C, Márki-Zay J, Schimmel H. Production of Certified Reference Materials (CRMs) for the analysis of the human Factor II (prothrombin) gene G20210A mutation. European Human Genetics Conference 6-9 May 2006, Amsterdam, The Netherlands

Abstract: Eur J Hum Genet 14;Suppl 1:375[P1256] (2006)

XII. Márki-Zay J, Gancberg D, Klein C, Schimmel H, Dux L. European proficiency testing study on the competence of laboratories to recognise rare mutations resulting in unexpected genotyping results. European Human Genetics Conference 6-9 May 2006, Amsterdam, The Netherlands

Abstract: Eur J Hum Genet 14;Suppl 1:379[P1276] (2006) 


\section{ACKNOWLEDGEMENTS}

These studies were supported by a ‘Training through Research’ Fellowship of the European Union.

I would like to thank my supervisors Dr. Christoph L. Klein and Prof. Dr. László Dux for giving me the topic and opportunity to perform this study and for all their help and support during these years.

I am very grateful to my former colleagues at the Institute of Reference Materials and Measurements, particularly to Dr. Heinz Schimmel, Dr. Thomas Linsinger, Dr. David Gancberg, Dr. Philipe Corbisier and Prof. Dr. Hendrik Emons, for their support and advices.

I wish to acknowledge our collaborators Prof. Dr. Christine Mannhalter and Dr. Sandra Rieger (Molecular Biology Division, Department of Clinical Chemistry and Laboratory Medicine, Medical University Vienna, Vienna, Austria), Prof. Dr. David Barton and Elizabeth Donohoe (National Centre for Medical Genetics, Our Lady’s Hospital for Sick Children, Crumlin, Ireland), Prof. Dr. Jean-Jacques Cassiman and Dr. Els Dequeker (Center for Human Genetics, Katholieke Universiteit Leuven, Leuven, Belgium), Dr. Glyn Stacey and Ross Hawkins (National Institute for Biological Standards and Control, Hertfordshire, UK), Prof. Dr. Rob Elles and Dr. Emma Gillaspy (National Genetics Reference Laboratory Manchester, Manchester, UK), Prof. Dr. Egbert Bakker and Dr. Francesco Russo (Department of Human and Clinical Genetics, Leiden University Medical Center, Leiden, The Netherlands), Prof. Dr. Michael Neumaier and Dr. Rolf Kruse (Reference Institute for Bioanalytics of the German Society for Laboratory Medicine, Bonn, Germany) Prof. Dr. Hans Reinauer and Ralf Flörke (Instand e.V. Düsseldorf, Germany) and Dr. Erika Sárkány (QualiCont In Vitro Diagnostic Quality Control Nonprofit Public Utility Ltd., Szeged, Hungary).

Finally I thank my family for all their support, understanding and patience. 


\section{LIST OF ABBREVIATIONS}

A

$\mathrm{AD}$

ANOVA Analysis of Variance

APC

ARML Accredited Reference Measurement Laboratory

BIPM International Bureau of Weights and Measures

Bp $\quad$ Base pairs

BSA Bovine serum albumin

C Cytosine

CAL Calibrator

CE (mark) Conformance mark according to the IVD Directive

CEN European Committee for Standardization

CF Cystic Fibrosis

CFTR Cystic Fibrosis Transmembrane conductance Regulator (gene)

CGPM General Conference on Weights and Measures

CLSI Clinical Laboratory Standards Institute

C-MBT Scientific Committee of Molecular Biology Techniques in Clinical Chemistry

Cp / Ct Crossing point / Cycle threshold

CRM Certified Reference Material

CRMGEN Acronym of the FP5 project 'Certified Reference Materials for Genetic Testing'

DMD Duchenne Muscular Dystrophy

DNA Desoxyribonucleic Acid

DTPA Diethylene Triamine Pentaacetic Acid

dNTP deoxy Nucleotide Triphosphate

EBV Epstein-Barr virus

EC $\quad$ European Commission;

EDTA Ethylenediaminetetraacetic acid

EMQN European Molecular Genetics Quality Network

EN European Norm 


\begin{tabular}{|c|c|}
\hline EQA & External Quality Assessment \\
\hline EQAS & External Quality Assessment Schemes \\
\hline ESTO & European Science and Technology Observatory Network \\
\hline EU & European Union \\
\hline FD & Freeze-dried \\
\hline FII & Human coagulation Factor II (prothrombin) \\
\hline FV & Human coagulation Factor V (proaccelerin) \\
\hline FVa & Activated human coagulation Factor V \\
\hline FMR1 & Fragile X mental retardation 1 (gene) \\
\hline FP & Research Framework Programme \\
\hline FraX & Fragile X \\
\hline FRET & Fluorescence Resonance Energy Transfer \\
\hline G & Guanine \\
\hline gDNA & Genomic DNA \\
\hline GMO & Genetically modified organism \\
\hline GUM & ISO Guide to the Expression of Uncertainty in Measurement, ISO, Geneva 1995 \\
\hline HFE & Human Hemochromatosis protein (gene) \\
\hline $\mathrm{HH}$ & Hereditary Hemochromatosis \\
\hline IEC & International Electrotechnical Commission \\
\hline IFCC & International Federation of Clinical Chemistry and Laboratory Medicine \\
\hline ILAC & International Laboratory Accreditation Cooperation \\
\hline IMEP & International Measurement Evaluation Programme \\
\hline IPR & Intellectual Property Right \\
\hline IPTS & Institute for Prospective Technological Studies (EC JRC) \\
\hline IRMM & Institute for Reference Materials and Measurements (EC JRC) \\
\hline ISO & International Organization for Standardization \\
\hline IVD & in vitro diagnostic \\
\hline JCTLM & Joint Committee for Traceability in Laboratory Medicine \\
\hline JRC & Joint Research Centre \\
\hline $\mathrm{LC}$ & LightCycler \\
\hline
\end{tabular}




\begin{tabular}{|c|c|}
\hline LDT & Laboratory Developed Test \\
\hline LOD & Limit of Detection \\
\hline LUMC & Leiden University Medical Centre \\
\hline LTSS & Long-term Stability Study \\
\hline ML & Manufacturer’s Laboratory \\
\hline NFD & Not freeze-dried \\
\hline NGRL & National Genetics Reference Laboratory \\
\hline NIBSC & National Institute for Biological Standards and Control \\
\hline NIST & National Institute of Standards and Technology (USA) \\
\hline NMI & National Metrology Institute \\
\hline NoE & Network of Excellence \\
\hline OECD & Organization for Economic Cooperation and Development \\
\hline PAGE & Polyacrylamide Gel Electrophoresis \\
\hline PCR & Polymerase Chain Reaction \\
\hline PEG & Polyethylene glycol \\
\hline Pfu & Pyrococcus furiosus (DNA polymerase) \\
\hline PT & Proficiency Testing \\
\hline QA & Quality Assurance \\
\hline QC & Quality Control \\
\hline QCM & Quality Control Material \\
\hline rDNA & Recombinant DNA \\
\hline RELA & IFCC EQA scheme for Reference Laboratories in Laboratory Medicine \\
\hline REMCO & ISO Committee on Reference Materials \\
\hline RfB & Reference Institute for Bioanalytics \\
\hline RFLP & Restriction Fragment Length Polymorphism \\
\hline RM & Reference Material \\
\hline RMP & Reference Measurement Procedure \\
\hline RMS & Reference Measurement System \\
\hline sDNA & Synthetic DNA \\
\hline SCA & Shared Cost Action (project type in the European framework programme) \\
\hline
\end{tabular}


SDS

Sodium Dodecyl Sulfate

SI

International System of Units

SNP

Single Nucleotide Polymorphism

SRM Standard Reference Material

SS DNA Salmon sperm DNA

STSS Short-term Stability Study

$\mathrm{T}$

Thymine

Taq

Thermus aquaticus (DNA polymerase)

TE

Tris-EDTA

$\operatorname{Tm}$

Melting temperature

Tris

Tris(hydroxymethyl)aminomethane

UK

United Kingdom

USA United States of America

VIM International Vocabulary of Metrology (ISO/IEC Guide 99:2007 corrected in 2010)

VTE Venous thromboembolism

WHO World Health Organization

wt

Wild-type 


\section{TERMS AND DEFINITIONS}

Unless indicated otherwise, metrological terms and definitions correspond to the ISO/IEC Guide 99:2007 International Vocabulary of Metrology - Basic and General Concepts and Associated Terms (corrected in 2010).

\section{Accuracy of measurement}

Closeness of agreement between a measured quantity value and a true quantity value of a measurand

\section{Analyte}

Component represented in the name of a measurable quantity (EN ISO 17511:2003).

\section{Allele}

One of the alternative forms of a gene that may occupy a given locus (CLSI document MM1-A2)

\section{Amplification}

The enzymatic replication in vitro of a target nucleic acid (CLSI document MM1-A2)

\section{Allele-specific oligonucleotide (ASO)}

A nucleic acid probe of short length, exactly complementary to either the normal or one of the mutant sequences of a target gene region, most often used for the detection of point mutations. (CLSI document MM1-A2)

\section{Assigned value}

Value attributed to a particular quantity and accepted, sometimes by convention, as having an uncertainty appropriate for a given purpose (EN 14136:2004)

\section{Calibrator}

Measurement standard used in calibration; Note: The term "calibrator” is only used in certain fields.

\section{Calibration}

Operation that, under specified conditions, in a first step establishes a relation between the quantity values with measurement uncertainties provided by measurement standards and corresponding indications with associated measurement uncertainties and, in a second step, uses this information to establish a relation for obtaining a measurement result from an indication.

\section{(Reference Material) Certificates and Supporting Reports}

Document accompanying a certified reference material stating one or more property values and their uncertainties, and confirming that the necessary procedures have been carried out to ensure their validity and traceability (ISO Guide 30:1992) 


\section{Certified Reference Material (CRM)}

Reference material characterized by a metrologically valid procedure for one or more specified properties, accompanied by a certificate that provides the value of the specified property, its associated uncertainty, and a statement of metrological traceability (ISO Guide 34:2009)

Note 1) The concept of value includes qualitative attributes, such as identity or sequence. Uncertainties for such attributes may be expressed as probabilities.

Note 2) Metrologically valid procedures for the production and certification of reference materials are given in, among others, ISO Guides 34 and 35.

Note 3) ISO Guide 31 gives guidance on the contents of certificates.

Note 4) VIM has an analogous definition (ISO/IEC Guide 99:2007, 5.14).

\section{Cloning}

Replication of a transferred gene or DNA segments in a host cell culture (CLSI document MM3-A2)

\section{Commutability of a reference material}

Property of a given RM demonstrated by the closeness of agreement between the relation among the measurement results, for a stated quantity in this material, obtained according to two given measurement procedures, and the relation obtained among the measurement results for other specified materials.

Note 1) The reference material in question is usually a calibrator and the other specified materials are usually routine samples.

Note 2) The measurement procedures referred to in the definition are the one preceding and the one following the reference material (calibrator) in question in a calibration hierarchy (see ISO 17511).

Note 3) The stability of commutable reference materials should be monitored regularly.

Working definition: interassay properties of a reference material, calibrator material, or quality control material that are comparable with those demonstrated by authentic clinical specimens (CLSI document C54-A)

\section{Complementary}

Describing the property of two strands of nucleic acid that can hybridize by specific base pairing between the nucleotides (CLSI document MM1-A2)

\section{Crossing point (Cp) / Cycle threshold (Ct)}

The Cp / Ct value is defined as the crossing point threshold and defines the threshold above which an amplification signal can be detected.

\section{External Quality Assessment (EQA) / Proficiency Testing}

Determination of individual and collective laboratory performance, and performance characteristics of examination procedures by means of interlaboratory comparison (EN 14136:2004)

Note: The primary objectives of EQA are educational, and can be supported by additional elements. 


\section{Genetic testing}

Genetic testing is testing for variations in germ line DNA sequences or products arising directly from changes in heritable genomic sequences that predict effects on the health, or influence the health management, of an individual. (OECD Guidelines for quality assurance in molecular genetic testing. OECD, 2007)

\section{Genotype}

The genetic makeup of an organism, or group of organism, with reference to a single trait, set of traits, or an entire complex of traits. (CLSI document MM1-A2)

\section{Heterozygous}

Having the two genes at corresponding loci on homologous chromosomes different for one or more loci (CLSI document MM17-A)

\section{Homozygous}

Having the two genes at corresponding loci on homologous chromosomes identical for one or more loci (CLSI document MM17-A)

\section{Hybridization}

Base pairing of complementary strands of nucleic acid by hydrogen bond formation, the binding of probe to specific nucleic acid sequences, or amplification products (CLSI document MM1-A2)

\section{Limit of Detection (LOD)}

Measured quantity value, obtained by a given measurement procedure, for which the probability of falsely claiming the absence of a component in a material is $\beta$, given a probability $\alpha$ of falsely claiming its presence.

\section{Measurand}

Quantity intended to be measured.

In genetic testing, it could be interpreted as the presence of a particular DNA sequence in the patient's blood. However, due to the various steps required during the analysis of the DNA (starting from the DNA extraction procedure from blood to further analysis such as PCR, RFLP or sequencing), the target actually measured at the final stage of analysis might differ from the one intended to be measured at the beginning as byproducts or impurities might have been added/removed from the original blood sample during the analytical procedure.

\section{Melting temperature (Tm)}

The melting temperature is the temperature at which $50 \%$ of the oligonucleotides are in doublestranded conformation and 50\% are single stranded.

\section{Mismatch}

Hybridization of two DNA or RNA strands that are less than $100 \%$ complementary (CLSI document MM1-A2) 


\section{Mutant}

A structural change in DNA sequence within a gene or chromosome resulting in the modification of an existing trait or creation of a new character or trait not found in the parental type (CLSI document MM17-A)

\section{Nominal property}

Property of a phenomenon, body, or substance, where the property has no magnitude

Example 1) Sex of a human being, Example 2) Colour of a paint sample, Example 3) Colour of a spot test in chemistry, Example 4) ISO two-letter country code, Example 5) Sequence of amino acids in a polypeptide.

Note 1) A nominal property has a value, which can be expressed in words, by alphanumerical codes, or by other means.

Note 2) 'Nominal property value' is not to be confused with nominal quantity value.

\section{Plasmid}

Small, ring-shaped DNA molecules that occur in bacteria and yeast outside the chromosome. Plasmids posses a so-called replicon, i.e. a DNA section which is required for the independent replication of the plasmid, and often carry an artificially inserted gene which renders the host bacterium resistant to certain antibiotics. (CLSI document MM3-A)

\section{Polymerase chain reaction (PCR)}

A common method of DNA amplification, utilizing pairs of oligonucleotide primers as start sites for repetitive rounds of DNA polymerase-catalyzed replication alternating with denaturation in successive heating-cooling cycles (CLSI document MM1-A2)

\section{Probe}

Defined piece of single stranded nucleic acid used to identify specific DNA OR RNA molecules bearing the complementary sequence; Note: The probe often carries a label (radioactive or chemical) so the probe can later be detected. (CLSI document MM1-A2)

\section{Production of a reference material}

All necessary activities and tasks leading to a reference material (certified or non-certified) supplied to customers (ISO Guide 34:2009)

Note 1) Production of a reference material includes production planning, production control, material handling and storage, material processing, assessment of homogeneity and stability, issue of statements and post-distribution service of the reference materials. It can include characterization, assignment of property values and their uncertainties, authorization and issue of certificates for certified reference materials.

Note 2) Production is not restricted to the manufacturing or preparation of the candidate materials. 


\section{Quantity}

Property of a phenomenon, body, or substance, where the property has a magnitude that can be expressed as a number and a reference. Note: A reference can be a measurement unit, a measurement procedure, a reference material, or a combination of such.

\section{Reference Material (RM)}

Material, sufficiently homogeneous and stable with respect to one or more specified properties, which has been established to be fit for its intended use in a measurement process

Note 1) RM is a generic term.

Note 2) Properties can be quantitative or qualitative, e.g., identity of substances or species. Note 3) Uses may include the calibration of a measurement system, assessment of a measurement procedure, assigning values to other materials, and quality control.

Note 4) A single RM cannot be used for both calibration and validation of results in the same measurement procedure.

Note 5) VIM has an analogous definition (ISO/IEC Guide 99:2007, 5.13), but restricts the term “'measurement'” to apply to quantitative values and not to qualitative properties. However, Note 3 of ISO/IEC Guide 99:2007, 5.13, specifically includes the concept of qualitative attributes, called “nominal properties”’. (ISO Guide 34:2009)

\section{Reference material producer}

Technically competent body (organization or company, public or private) that is fully responsible for project planning and management, assignment of and decision on property values, authorization of property values and issue of the certificate or other statements for the reference materials it produces (ISO Guide 34:2009)

\section{Reference measurement procedure}

Measurement procedure accepted as providing measurement results fit for their intended use in assessing measurement trueness of measured quantity values obtained from other measurement procedures for quantities of the same kind, in calibration, or in characterizing reference materials

\section{Reference method}

A thoroughly investigated method, clearly and exactly describing the necessary conditions and procedures, for the measurement of one or more property values that has been shown to have accuracy and precision commensurate with its intended use and that can therefore be used to assess the accuracy of other methods for the same measurement, particularly in allowing the characterisation of a reference material. (ISO Guide 30:1992)

\section{Repeatability}

Measurement precision under a set of repeatability conditions of measurement 


\section{Repeatability condition}

Condition of measurement, out of a set of conditions that includes the same measurement procedure, same operators, same measuring system, same operating conditions and same location, and replicate measurements on the same or similar objects over a short period of time

\section{Recombinant DNA (rDNA)}

DNA artificially constructed by combining genes from different organisms or by cloning chemically altered DNA, usually for the purpose of genetic manipulation. (Unified Medical Language System (UMLS): http://www.nlm.nih.gov/research/umls/)

\section{Restriction enzyme}

Restriction endonuclease; any of a large number of bacterial enzymes that cleave double-stranded DNA at specific nucleotide sequences (CLSI document MM1-A2)

\section{(Analytical) Specificity}

Ability of a measurement procedure to measure solely the measurand (EN ISO 17511:2003)

\section{(Analytical) Sensitivity}

Quotient of the change in an indication of a measuring system and the corresponding change in a value of a quantity being measured

\section{Single nucleotide polymorphisms (SNPs)}

A sequence variant involving a change in a single nucleotide; Note: The term is often used broadly to encompass any single-nucleotide change, including point mutations. (CLSI document MM1-A2)

\section{Stability}

Property of a measuring instrument, whereby its metrological properties remain constant in time

\section{Standard Reference Material (SRM)}

Certified reference materials issued by the National Institute of Standards and Technology (NIST);

\section{(Metrological) Traceability}

Property of a measurement result whereby the result can be related to a stated metrological reference through a documented unbroken chain of calibrations, each contributing to the measurement uncertainty

Note 1) For this definition, a 'stated metrological reference' can be a definition of a measurement unit through its practical realization, or a measurement procedure including the measurement unit for a non-ordinal quantity, or a measurement standard.

Note 2) Metrological traceability requires an established calibration hierarchy.

Note 3) Specification of the reference must include the time at which this reference was used in establishing the calibration hierarchy, along with any other relevant metrological information about the reference, such as when the first calibration in the calibration hierarchy was performed. 
Note 4) For measurements with more than one input quantity to the measurement model, each of the input quantities should itself be metrologically traceable and the calibration hierarchy involved may form a branched structure or a network. The effort involved in establishing metrological traceability for each input quantity value should be commensurate with its relative contribution to the measurement result.

Note 5) Metrological traceability of a measurement result does not ensure that the measurement uncertainty is adequate for a given purpose or that there is an absence of mistakes.

Note 6) A comparison between two measurement standards may be viewed as a calibration if the comparison is used to check and, if necessary, correct the quantity value and measurement uncertainty attributed to one of the measurement standards.

Note 7) The ILAC considers the elements for confirming metrological traceability to be an unbroken metrological traceability chain to an international measurement standard or a national measurement standard, a documented measurement uncertainty, a documented measurement procedure, accredited technical competence, metrological traceability to the SI, and calibration intervals (see ILAC P-10:2002).

Note 8) The abbreviated term ‘traceability' is sometimes used to mean 'metrological traceability' as well as for other concepts, such as ‘sample traceability' or 'document traceability' or 'instrument traceability' or 'material traceability', where the history ('trace') of an item is meant. Therefore, the full term of 'metrological traceability' is preferred if there is risk of confusion.

\section{Traceability chain}

Sequence of measurement standards and calibrations that is used to relate a measurement result to a reference

\section{Trueness}

Closeness of agreement between the average value obtained from a large series of test results and an accepted reference value.

\section{(Measurement) Uncertainty}

Non-negative parameter characterizing the dispersion of the quantity values being attributed to a measurand, based on the information used

Note 1) Measurement uncertainty includes components arising from systematic effects, such as components associated with corrections and the assigned quantity values of measurement standards, as well as the definitional uncertainty. Sometimes estimated systematic effects are not corrected for but, instead, associated measurement uncertainty components are incorporated.

Note 2) The parameter may be, for example, a standard deviation called standard measurement uncertainty (or a specified multiple of it), or the half-width of an interval, having a stated coverage probability. 
Note 3) Measurement uncertainty comprises, in general, many components. Some of these may be evaluated by Type A evaluation of measurement uncertainty from the statistical distribution of the quantity values from series of measurements and can be characterized by standard deviations. The other components, which may be evaluated by Type B evaluation of measurement uncertainty, can also be characterized by standard deviations, evaluated from probability density functions based on experience or other information.

Note 4) In general, for a given set of information, it is understood that the measurement uncertainty is associated with a stated quantity value attributed to the measurand. A modification of this value results in a modification of the associated uncertainty.

\section{Validation}

Verification, where the specified requirements are adequate for an intended use.

Example: A measurement procedure, ordinarily used for the measurement of mass concentration of nitrogen in water, may be validated also for measurement in human serum.

\section{Verification}

Provision of objective evidence that a given item fulfils specified requirements.

Example 1) Confirmation that a given reference material as claimed is homogeneous for the quantity value and measurement procedure concerned, down to a measurement portion having a mass of $10 \mathrm{mg}$.

Example 2) Confirmation that performance properties or legal requirements of a measuring system are achieved.

Example 3) Confirmation that a target measurement uncertainty can be met.

Note 1) When applicable, measurement uncertainty should be taken into consideration.

Note 2) The item may be, e.g. a process, measurement procedure, material, compound, or measuring system.

Note 3) The specified requirements may be, e.g.; that a manufacturer's specifications are met.

Note 4) Verification in legal metrology, as defined in VIM, and in conformity assessment in general, pertains to the examination and marking and/or issuing of a verification certificate for a measuring system.

Note 5) Verification should not be confused with calibration. Not any verification is a validation.

Note 6) In chemistry, verification of identity of entity involved, or of activity, requires a description of the structure or properties of that entity or activity.

\section{Wild-type}

The normal, as opposed to the mutant, gene or allele (CLSI document MM17-A) 


\section{TABLE OF CONTENTS}

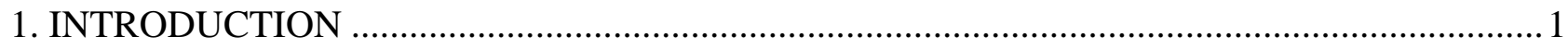

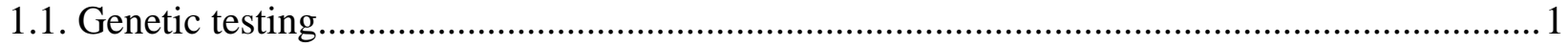

1.2. Reference measurement systems in the laboratory medicine ........................................... 3

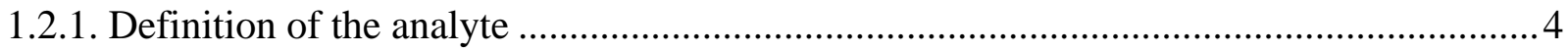

1.2.2. Reference Measurement Procedure …......................................................................

1.2.3. Reference materials, certified reference materials ....................................................6

1.2.4. Reference laboratories (reference measurement services) ........................................... 10

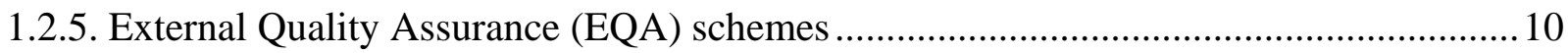

1.3. Regulation of genetic testing - Role of EU/EC with the IVD Directive ............................11

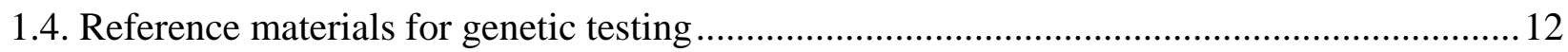

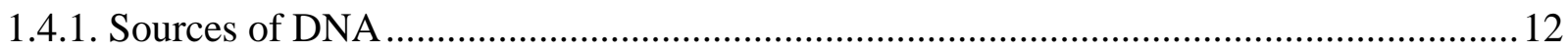

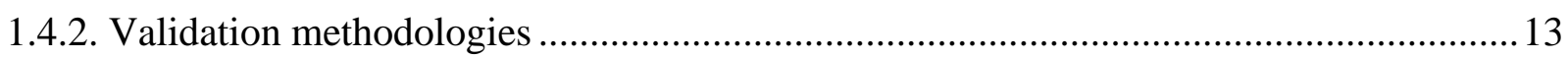

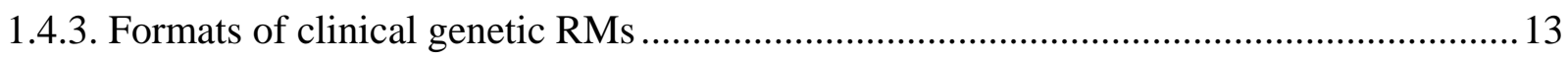

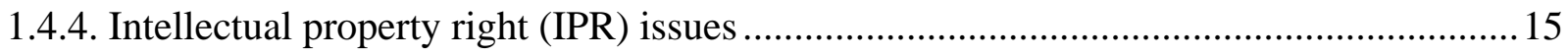

1.4.5. Priorities in the development of CRMs for molecular genetic testing............................ 15

1.5. Initiatives for the development of (Certified) Reference materials for genetic testing ..........17

1.5.1. The Certified Reference Materials for Molecular Genetic Testing (CRMGEN) project 17

1.5.2. Development of CRMs for the identification of the human Factor II G20210A variant

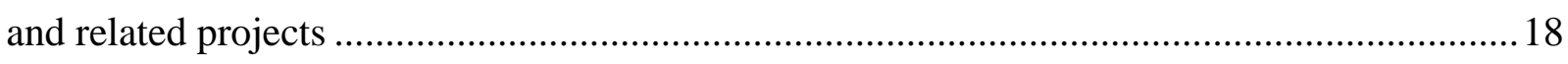

1.5.3. The EuroGenTest Network of Excellence project ...................................................... 18

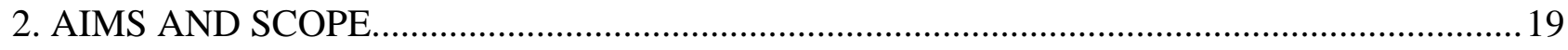

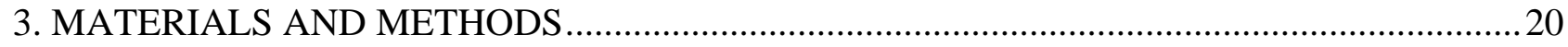

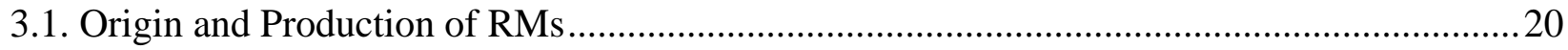

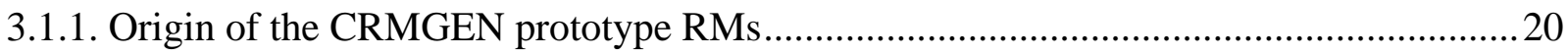

3.1.2. Origin of the NIBSC-WHO candidate Reference Panel samples ................................... 22

3.1.3. Preparation of prototype RMs for the feasibility study to optimise DNA preservation ..22

3.1.4. Production of the candidate CRMs IRMM/IFCC-490, -491 and -492 and the proficiency testing samples for the analysis of the prothrombin G20210A variant....................................23

3.1.5. Freeze-drying protocol developed for the lyophilisation of DNA-based RMs................224

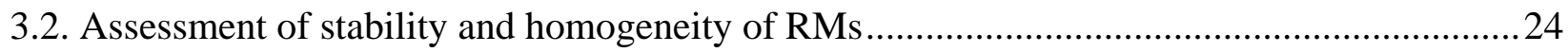

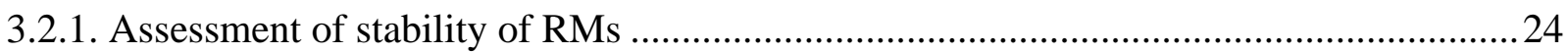

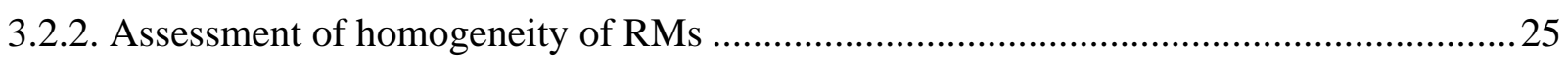

3.2.3. Assessment of the freeze-thaw tolerance of prototype RMs .........................................25 


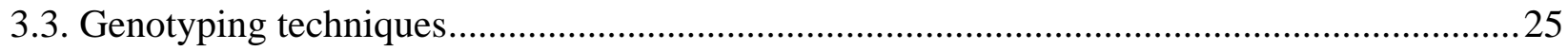

3.3.1. Sample preparation and DNA quantitation by PicoGreen .............................................25

3.3.2. Analysis of the C282Y and H63D mutations in the human HFE gene ..........................26

3.3.3. Genotyping of the FMR1 locus in the CRMGEN-FraX prototype RMs........................27

3.3.4. Analysis of the human coagulation Factor V G1691 “Leiden” mutation ........................27

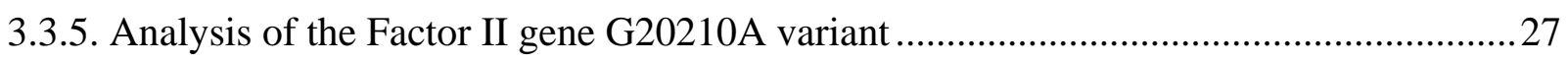

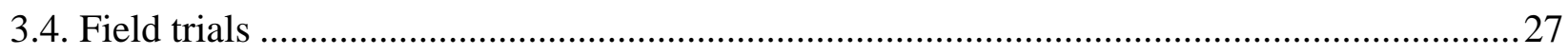

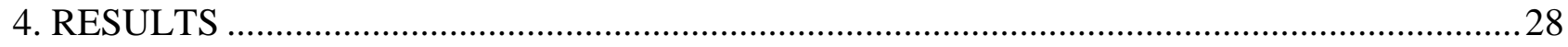

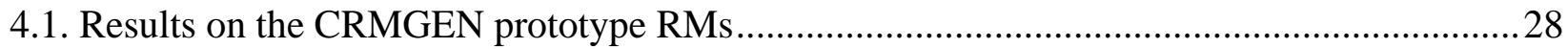

4.1.1. CRMGEN-HH - PCR product based prototype RMs .................................................28

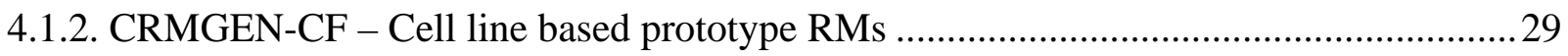

4.1.3. CRMGEN-FraX - Genomic DNA based prototype RMs ..........................................29

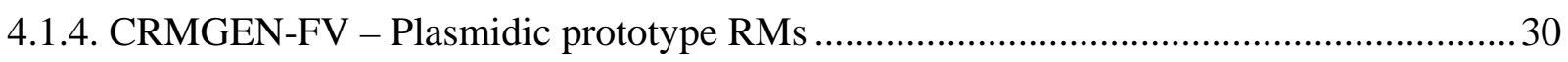

4.2. Analysis of the NIBSC-WHO candidate Reference Panel samples .................................... 32

4.3. Feasibility study on the preservation of plasmidic DNA …...................................................33

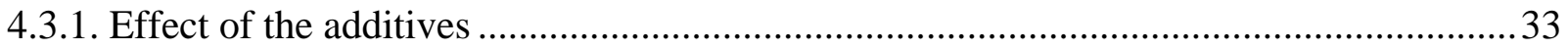

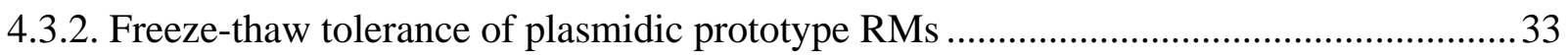

4.4. Production and certification of the candidate CRMs IRMM/IFCC-490, -491 and $-492 \ldots . . . .33$

4.4.1. Construction and characterisation of the reference plasmids.........................................33

4.4.2. Evaluation of the $1^{\text {st }}$ batch of the candidate FII CRMs................................................3

4.4.3. Characterisation of the new lot of the CRMs IRMM/IFCC-490, -491 and $-492 \ldots . . . . . . . . .35$

4.5. Proficiency testing study using QCMs containing rare sequence variants ............................38

4.5.1. Preparation of the samples for the proficiency testing exercise .....................................38

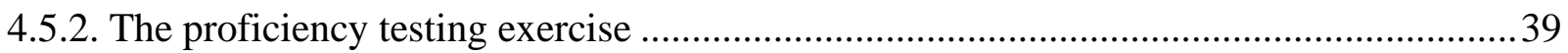

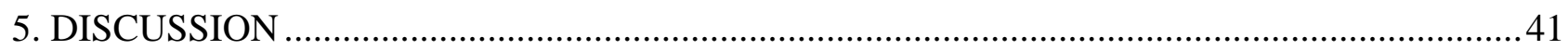

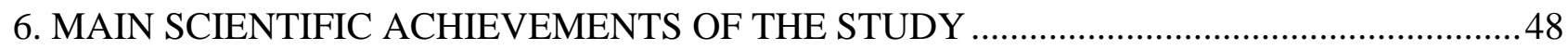

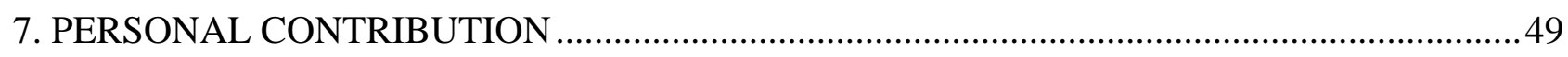

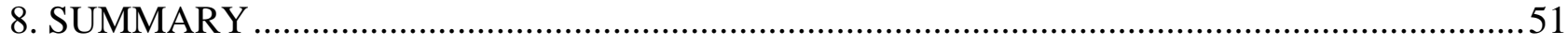

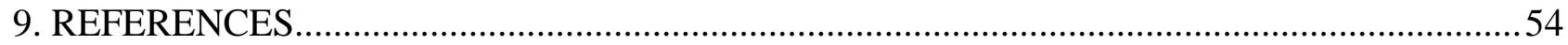




\section{INTRODUCTION}

Recent scientific and technological advances, such as the invention of the polymerase chain reaction (PCR) technique and the deciphering of the human genome, revealed the genetic background of medical conditions and led to an enormous progress in our knowledge on the association between specific sequence variants and particular disease entities. On the other hand, many of the molecular biology techniques developed and applied originally for scientific purposes meet the requirements for routine diagnostic utilization regarding their robustness and performance characteristics. Such molecular diagnostic assays are able to detect genetic alterations resulting in inheritable disorders, higher risk to develop diseases or altered drug metabolism and play an increasingly important role in the diagnosis, in risk stratification and even more also in the choice of the right treatment for the patients. These tests are highly specific and thousands of different tests are already in routine use. DNA test results can be predictive for future onset of disorders prior to the manifestation of clinical symptoms, and thus have significant implications for the individual patient as well as for his/her relatives.

Although public perception holds that genetic tests are highly accurate and represent a state-of-the-art technology, the development of systems to ensure the quality of methods and testing services has lagged behind the spread of application of DNA-based tests in medicine. Concerns over the accuracy of genetic test results were further supported by several studies $[1,2,3,4,5]$ and publications of international organisations [6,7] reporting high error rates and lack of standardisation and available certified reference materials (CRMs) for clinical genetic testing. Quality issues in genetic testing are given added importance because individuals are usually only tested once in their lifetime, and an incorrect genotyping result may remain hidden for many years with dramatic consequences for the tested person. These facts combine to make standardisation and improvement of quality in molecular genetic testing a matter of utmost importance.

\subsection{Genetic testing}

There exist slightly different definitions of "genetic testing”, regarding the comprehensiveness of what is subsumed under this term [8]; however, the working definition used by OECD [9] is widely accepted and fits into the scope of the present study as well: "Genetic testing is testing for variations in germ line DNA sequences or products arising directly from changes in heritable genomic sequences that predict effects on the health, or influence the health management, of an individual." However, the current thesis targeted only molecular testing of germline DNA sequences, excluding other types of diagnostic tests, such as cytogenetics or biochemical testing. Molecular genetic tests as defined above can be employed for the following purposes in the laboratory medicine: 
- Diagnostic testing: to confirm, refine or exclude a clinical diagnosis.

- Predictive testing: to estimate the risk to a person with no symptoms of developing a genetic (presymptomatic testing), or a multifactorial disorder (predisposition testing) in the future; or increasingly to predict drug response (pharmacogenetics).

- Carrier testing: to clarify the presence of a gene mutation for a recessively inherited or Xlinked disorder, this might be important for reproductive decisions.

- Prenatal testing and preimplantation genetic diagnosis: to clarify whether the foetus or embryo at high risk for a hereditary disease carries the particular mutations or alterations responsible the disease.

- Genetic screening: Diagnostic testing, predictive testing, prenatal testing and carrier testing offered systematically at the population level or for sub-populations defined on the basis of their risk.

Another widespread clinical application of DNA-based testing is carried out for disease sub-typing in oncology to characterize different types of cancer; however, in these cases somatic mutations are investigated, which arise in a specific tissue, causing a clone of cells to proliferate.

Although a few genetic tests are quantitative, such as the measurement of the number of copies of a gene or part of a gene (to determine the relative copy number of a gene or the numerical changes in tandem repeats), most molecular genetic tests are qualitative in nature - their purpose is to determine a particular DNA sequence in a patient's sample. This approach differs fundamentally from other, so called qualitative tests used in the laboratory medicine, which have the ability to distinguish positive and negative sample populations by applying a cut off value or by setting up the measurement procedure in a way that it gives at a certain concentration level of the analyte a detectable signal can be best described by the limit of detection, which is a quantity value.

In some cases, genotyping techniques are designed for accurate and rapid detection of known variants (mutation testing) while in other cases - typically in monogenic disorders - the sample is screened for any deviation from the normal sequence (mutation scanning). There are a large number of approaches for both mutation testing and mutation scanning. The situation is further complicated by the existence of many different types of sequence variants, (single nucleotide polymorphisms (SNPs), deletions, gene rearrangements, duplications, triplet repeat expansions, etc.) each of which may require different testing or scanning technologies. There is no reference or 'gold standard' testing or scanning method which will detect all possible mutation types.

Genetic alterations and their association to pathological conditions are usually discovered by research laboratories. When these relationships have been clearly identified and confirmed, and the indications of testing and therapeutic consequences of the results have been clarified, the scientific evidence can be utilised also in the patient care $[7,9,10]$. 
In contrast to the thousands of molecular genetic markers identified, the number of commercial kits available for genetic testing is rather limited and the vast majority of genotyping assays is carried out using individual reagents according to an individual procedure adapted from the literature or developed in-house by the individual laboratory. These tests are usually referred as 'home-brew' or 'laboratory/in-house developed tests'. The performance (measured in terms of specificity and robustness) of such laboratory developed tests (LDTs) varies from laboratory to laboratory [11], because they are not always subjected to the same quality standards as the commercial kits and do not come under the market vigilance systems of the competent authorities. If the number of the performed tests exceeds a certain limit, also commercial kits appear on the market, which are usually more rigorously investigated for their performance and quality.

Recommendations and guidelines to be followed for the clinical use (e.g. indications, therapeutic consequences, counselling) and for the diagnostic testing (recommended methods, assay conditions, quality control, reporting, etc.) can be drafted just after several year of clinical application and many hundred thousand of performed tests, if the clinical and diagnostic experiences reach a critical limit. These shortcomings are partially caused by the lack of appropriate reference materials (e.g. positive and negative controls), which are required for the proper validation and quality assurance (QA) of the molecular genetic tests. However, except of polymorphisms, sequence variants are infrequent; therefore thousands of tests might be carried out without ever encountering a positive result.

Many molecular genetic tests are performed in specialist or clinical research laboratories with substantial expertise in the field [7,9]. These laboratories play a valuable role in the development and validation of new tests as well as in the characterisation of mutations in rare diseases; however, they usually do not measure up to the same regulations and norms relating to in vitro diagnostic centres $[9,10]$. On the other hand, common variants are now increasingly being tested in routine clinical laboratories, which are more familiar with the quality assurance of measurement procedures.

\subsection{Reference measurement systems in the laboratory medicine}

In order to achieve and ensure the highest level of accuracy in the clinical laboratory measurements, a reference measurement system (RMS) have to be established [12,13,14,15,16,17]. Reference measurement systems are needed for producing useful and reliable measurement results in the routine diagnostic services as well as in science and technology, which are comparable among laboratories and ultimately traceable to measurement standards of the highest metrological level [18].

Traceability is defined according to the International Vocabulary of Basic and General Terms in Metrology [19] as a property of the result of a measurement or the value of a standard, whereby it 
can be related to higher-order reference materials or reference methods, through an unbroken and well-characterised chain of comparisons all having stated uncertainties as presented in the standard EN ISO 17511:2003 [15] (Figure 1.). In order to ensure this unbroken traceability chain, analytical specificity of the measurement procedures and commutability of the calibrators are essential.

Figure 1: Calibration hierarchy according to one scheme given in EN ISO 17511:2003 [15]

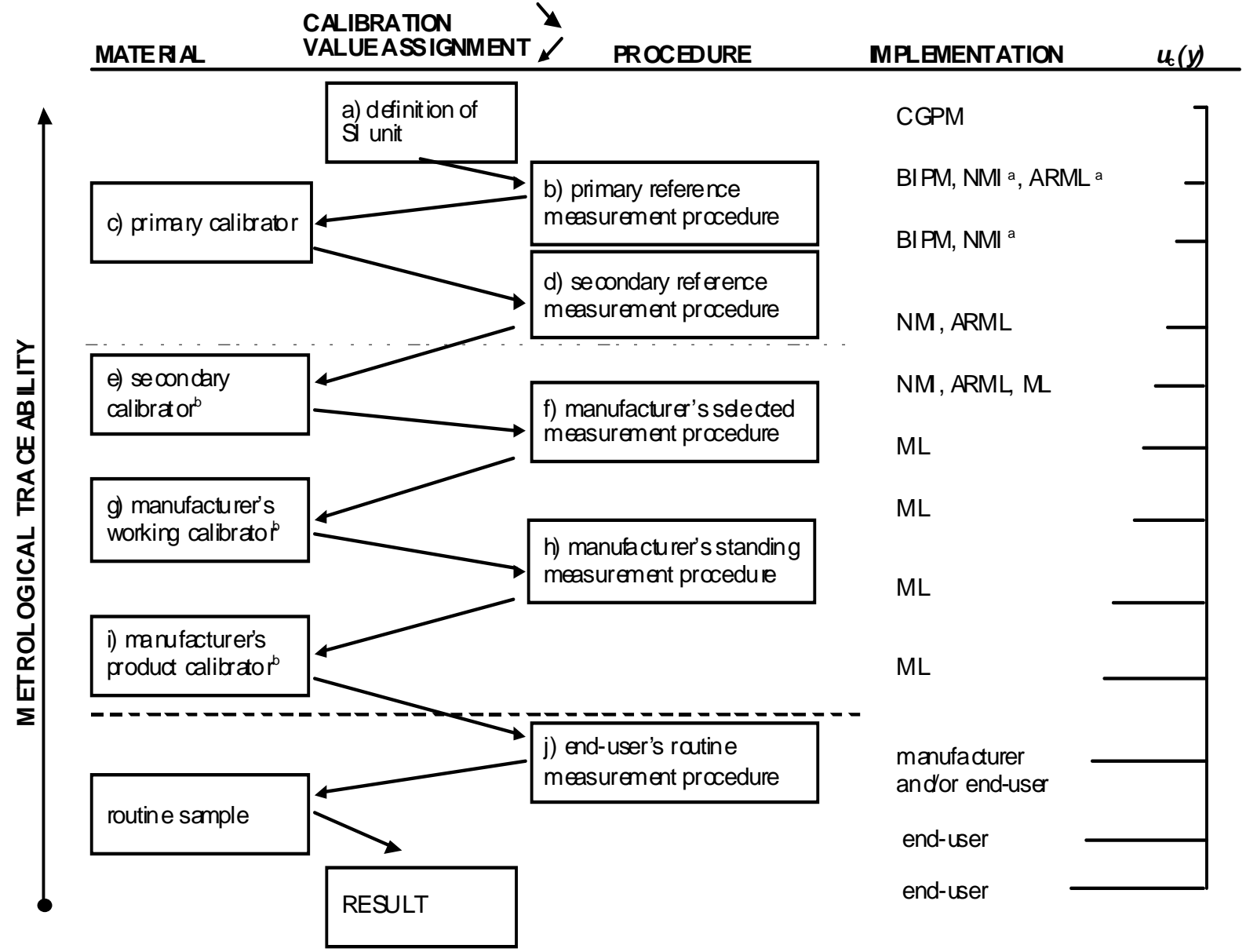

The three elements of RMSs are internationally accepted and commonly agreed reference measurement procedures (RMP), certified reference materials (CRM) and reference laboratories possibly collaborating in a network. The standard EN ISO 17511:2003 [15] also refers to the RMS and requires for measurement of quantities in biological samples: (i) definition of the analyte to be measured in human samples with regard to the intended use of the measurement results, (ii) RMP for the selected quantity in the samples, and (iii) suitable reference materials for the selected quantity, e.g. primary calibrators and secondary matrix-based calibrators that are commutable. Successful implementation of such systems can be further enhanced by External Quality Assurance (EQA) programmes [16,20] .

\subsubsection{Definition of the analyte}

Clinical laboratory testing typically involves the determination of a known analyte in a complex matrix. The vast majority of clinically relevant analytes are biological macromolecules and in many 
cases complex mixtures of related isoforms which are present at low concentration levels. It is of pivotal importance to understand the biomedical functionality to select and address the clinically relevant macromolecules/isoforms [21,22]. A clear definition of the analyte with regard to the intended clinical use of the measurement results is a necessary prerequisite for performing such determinations as well as to set up of a RMS [21,22]. Consequently the measurement procedure chosen and the properties of the reference materials used for calibration have a pronounced effect on the degree of standardisation, which can be achieved.

Molecular genetic testing investigates the identity or property of specific nucleotide sequence(s) using analytical methods, which require already an exact description of the DNA sequence of interest. However, in a few cases, medical diagnosis needs additional characterisation of the target sequence (e.g. the methylation status in fragile $\mathrm{X}$ syndrome).

\subsubsection{Reference Measurement Procedure}

A reference measurement procedure (RMP) is a well characterised and extensively evaluated procedure, which specifically measures the analyte as defined [17,19]. The RMP consists of a reference method and national/international guidelines and recommendations. The key characteristic expected from a reference method is that the method should be robust and not affected by small changes in the temperature, $\mathrm{pH}$, concentrations of reagents, etc. In principle, a measurement procedure shall have been validated by inter-laboratory studies before final acceptance as a measurement procedure $[17,18]$.

In the RMS, the RMP is applied to assign a certified value to a given reference material and to assess the performance characteristics of different routine measurement systems $[17,18]$ comprising measuring instruments, auxiliary equipment and reagents. Furthermore, RMP can be used for the evaluation of functional interchangeability of different routine measurement procedures purporting to measure the same quantity, and for the recognition of analytical interferences in patient samples. In genetic testing, several national/international best practice guidelines have been developed for particular diseases as well as for molecular genetic testing in general [23,24,25]; but reference method has not yet been established and installed in the area [26,27]. If no reference method has been approved, a designated comparison method can be employed. For instance, sequencing is a quite robust technique in SNP genotyping [26,27,28] and bi-directional sequencing analysis is widely accepted as a 'gold standard' method in case of dubious results [26,27].

On the other hand, to become approved as a reference method for SNPs, sequencing should be validated according to the internationally accepted requirements for reference methods. Except for preimplantation genetic diagnosis, the analytical sensitivity and detection limits of the methods used in genetic testing do not cause major issues. However, the specificity and the accuracy of the method are of pivotal importance and have to be evaluated by inter-laboratory studies: Uniform 
DNA samples would have to be dispatched to many laboratories for sequencing, and the results should be similar using different platforms and chemistries on different days. In addition, to assess the probability of sequencing failures under the requirement of matching forward and backward sequencing, a huge number of sequencing assays should be carried out to detect the expected low number of matching misreading [26].

\subsubsection{Reference materials, certified reference materials}

(Certified) Reference Materials are generally recognised as an excellent mean to check analytical accuracy and create crucial reference points in the process of the development of comprehensive measurement systems [13]. Applications of RMs may include the development and validation of a measurement procedure (in particular evaluation of trueness and estimation of uncertainty), the calibration of a measurement system, the assignment of traceable values to other materials, and quality control [15]. Choosing a qualified material like a CRM offers the practical advantage that it dispenses the user from having to establish crucial QA material characteristics himself. Moreover, the traceable assigned values allow together with the uncertainty statement an assessment of trueness - and consequently accuracy - of analytical results. In addition, issuing of a certificate can be seen as signalling a commitment to highest production quality and therefore increases confidence in the stated CRM values [21,29]. In vitro diagnostic (IVD) manufacturers use also CRMs to assure the traceability of values assigned to their calibrators and quality control materials and for the evaluation of their routine measurement procedures. Thus, the availability and use of matrix-based CRMs is generally seen as a critical step in the standardisation process of any clinical analyte $[13,21]$.

The type of RM which is required for a measurement procedure depends on the analytical problem. An analysis which is directed to a qualitative property, such as the nucleotide sequence, needs a RM which allows comparison of this qualitative property in the sample and in the RM during the application of the same testing procedure [26]. On the other hand, quantitative measurements have to be calibrated in order to establish the relation between the targeted quantity in the sample and the measurement signal, which requires calibration materials with known property values [26]. Finally, reliability of the measurement results can only be assured if the performance of the whole analytical process is verified using appropriate quality control materials. Therefore, so-called matrix RMs are developed which mimic as closely as possible the real clinical samples (e.g. blood, urine, cerebrospinal fluid) and permit the quality assurance also of the sample preparation, not only the quantification step.

In 2005, a new definition [30] of the term 'reference material' has been accepted by the Committee on Reference Materials (REMCO), which was slightly revised in 2009 [31]. This definition states that an RM is a "Material, sufficiently homogeneous and stable with respect to one or more 
specified properties, which has been established to be fit for its intended use in a measurement process. Note 1: RM is a generic term. Note 2: Properties can be quantitative or qualitative, e.g., identity of substances or species. Note 3: Uses may include the calibration of a measurement system, assessment of a measurement procedure, assigning values to other materials, and quality control. Note 4: A single RM cannot be used for both calibration and validation of results in the same measurement procedure. Note 5: VIM has an analogous definition (ISO/IEC Guide 99:2007, 5.13), but restricts the term "measurement" to apply to quantitative values and not to qualitative properties. However, Note 3 of ISO/IEC Guide 99:2007, 5.13, specifically includes the concept of qualitative attributes, called "nominal properties" [31].

Figure 2. The 'RM family’

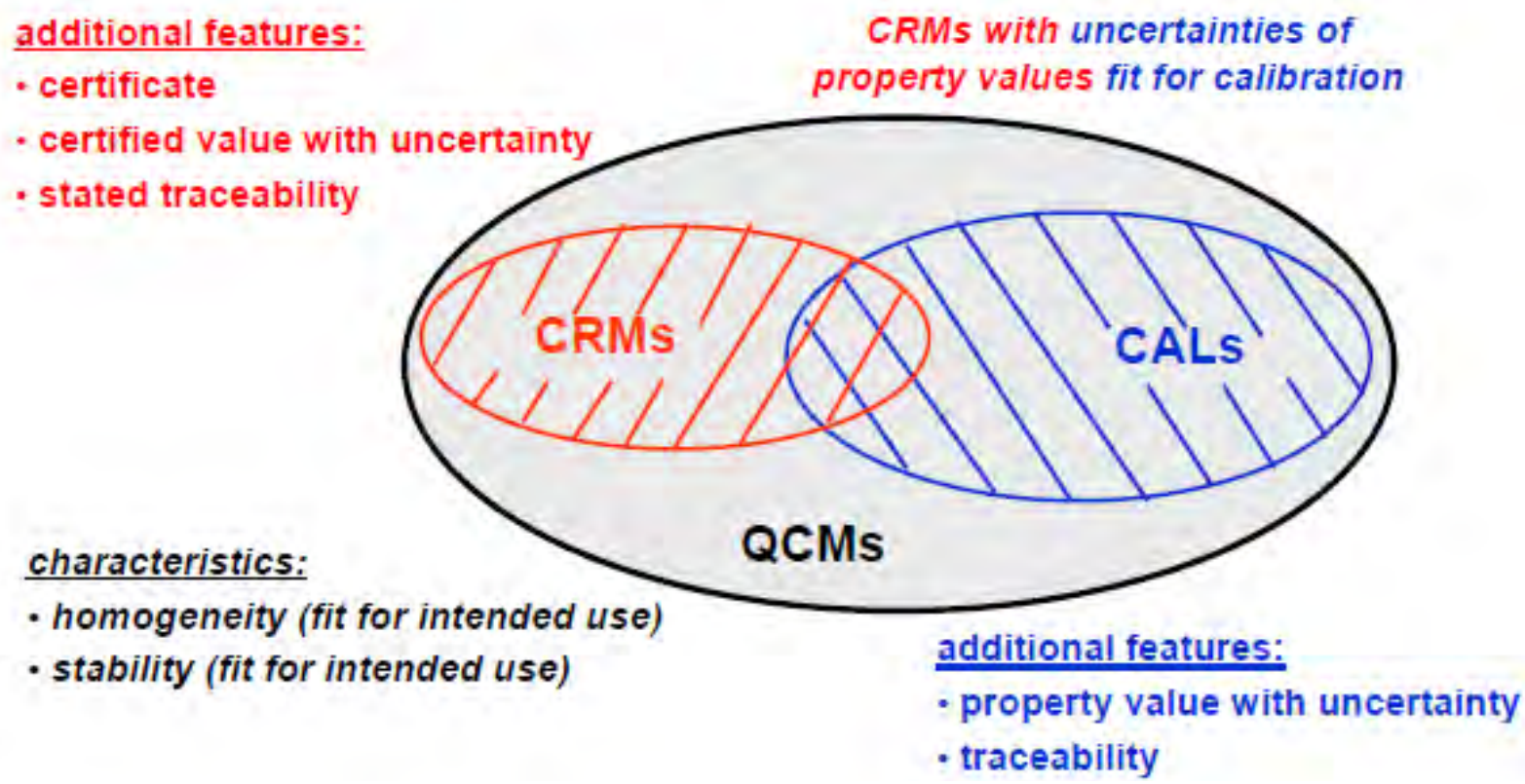

The first note emphasizes the fact that the term 'reference material' should be understood as a family name covering different types of RMs [32], such as Quality Control Materials (QCMs), Calibrators and CRMs (Figure 2.). QCMs do not need additional features beyond the fundamental requirements for any type of RMs. Calibrators represent a subgroup of RMs, which can be used for the calibration of test systems through the assigned property value with uncertainty and traceability to available reference measurement procedures and/or available reference materials of a higher order. Certified reference materials is a subgroup of RMs as well, which is now defined as "Reference material, characterized by a metrologically valid procedure for one or more specified properties, accompanied by a certificate that provides the value of the specified property, its associated uncertainty, and a statement of metrological traceability. Note 1: The concept of value includes qualitative attributes such as identity or sequence. Uncertainties for such attributes may be expressed as probabilities. Note 2: Metrologically valid procedures for the production and certification of reference materials are given in, among others, ISO Guides 34 and 35. Note 3: ISO 
Guide 31 gives guidance on the contents of certificates. Note 4: VIM has an analogous definition (ISO/IEC Guide 99:2007, 5.14).” [31]

Main steps in the production of CRM include the design of a RM depending on its intended use, the selection of an appropriate starting material, a feasibility study for processing and characterising the material, the preparation of the candidate RM, a homogeneity study, short- and long-term stability studies, the characterisation of the candidate RM with respect to the target properties and evaluation of performance by the intended users (e.g. clinical laboratories) in situations where most likely will be used, the assignment of the certified values and their uncertainties, and finally, the issuing of a certificate [29,31] that states the value of the specified property, its associated uncertainty and a statement of metrological traceability [30,32]. The content of the certificate is defined in the ISO Guide 31 [33]. The general procedure of CRM production is presented in Figure 3., while the Figure 4. shows the scheme of a typical certification campaign.

Figure 3. General Procedure for CRM Production

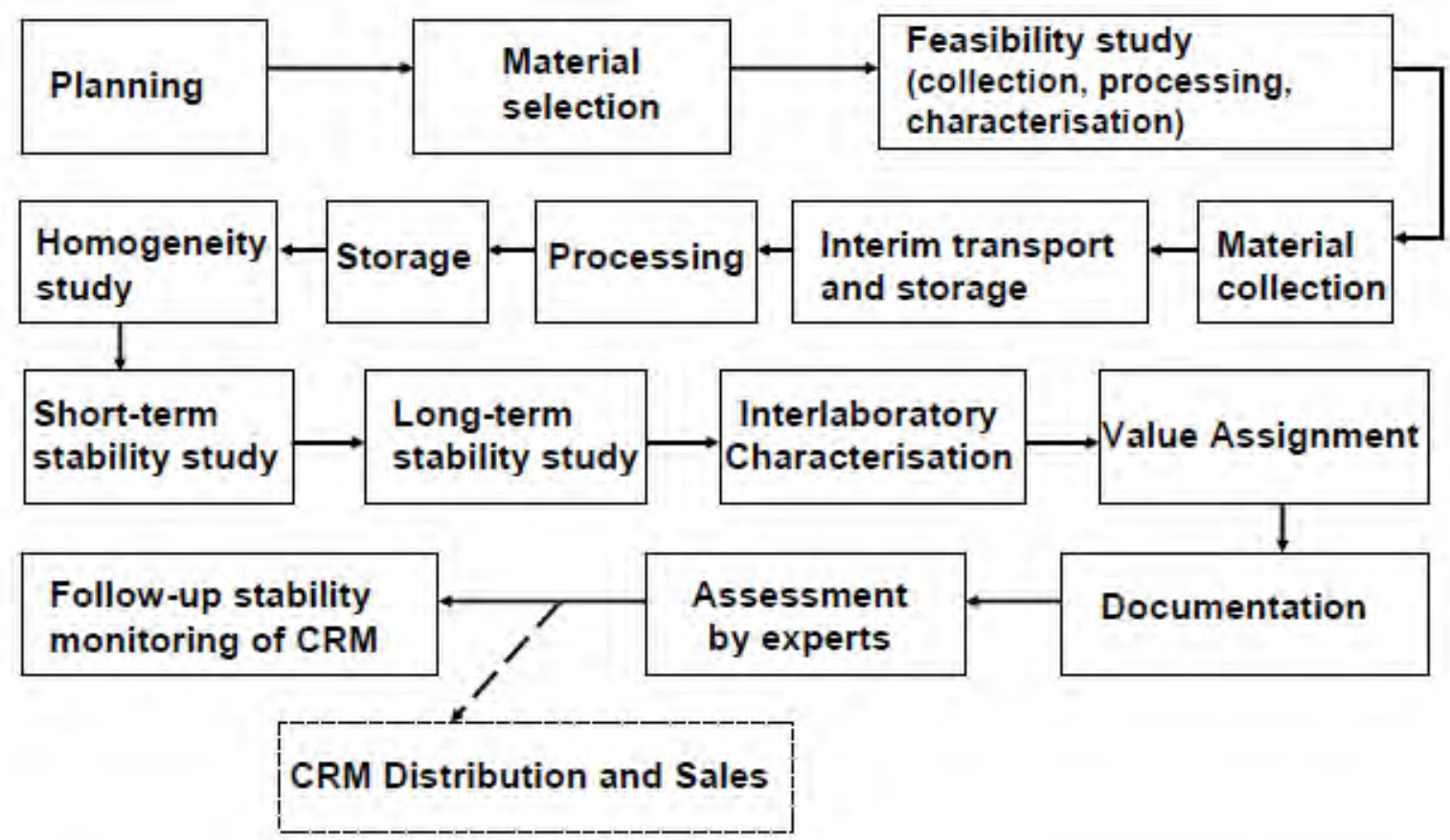

RMs require by definition extensive studies to guarantee their homogeneity, stability and fitness for purpose [34]. The homogeneity of a RM refers to both the homogeneity of the material within one unit and the homogeneity between units. Within-unit homogeneity testing determines the minimum sample intake, for which the reported uncertainty is still valid and between-unit homogeneity testing deals with the differences among samples, which contribute to the uncertainty of the certified value. Ideally, stability studies on RMs should follow a so called isochronous scheme so that all samples are measured under repeatability conditions [35]. The idea of this real-time scheme is that stability status of a material can be "frozen" at so called 'reference conditions' and the measurements of complete study can be carried out at the same time. While a short-term stability study (STSS) aims 
at determining the optimum transport conditions of the materials, long-term stability is a crucial parameter for estimating the shelf-life of a (C)RM under certain storage conditions. Stability of CRMs has to be followed up during their whole life time (so called post-certification monitoring).

Figure 4. Typical certification campaign scheme

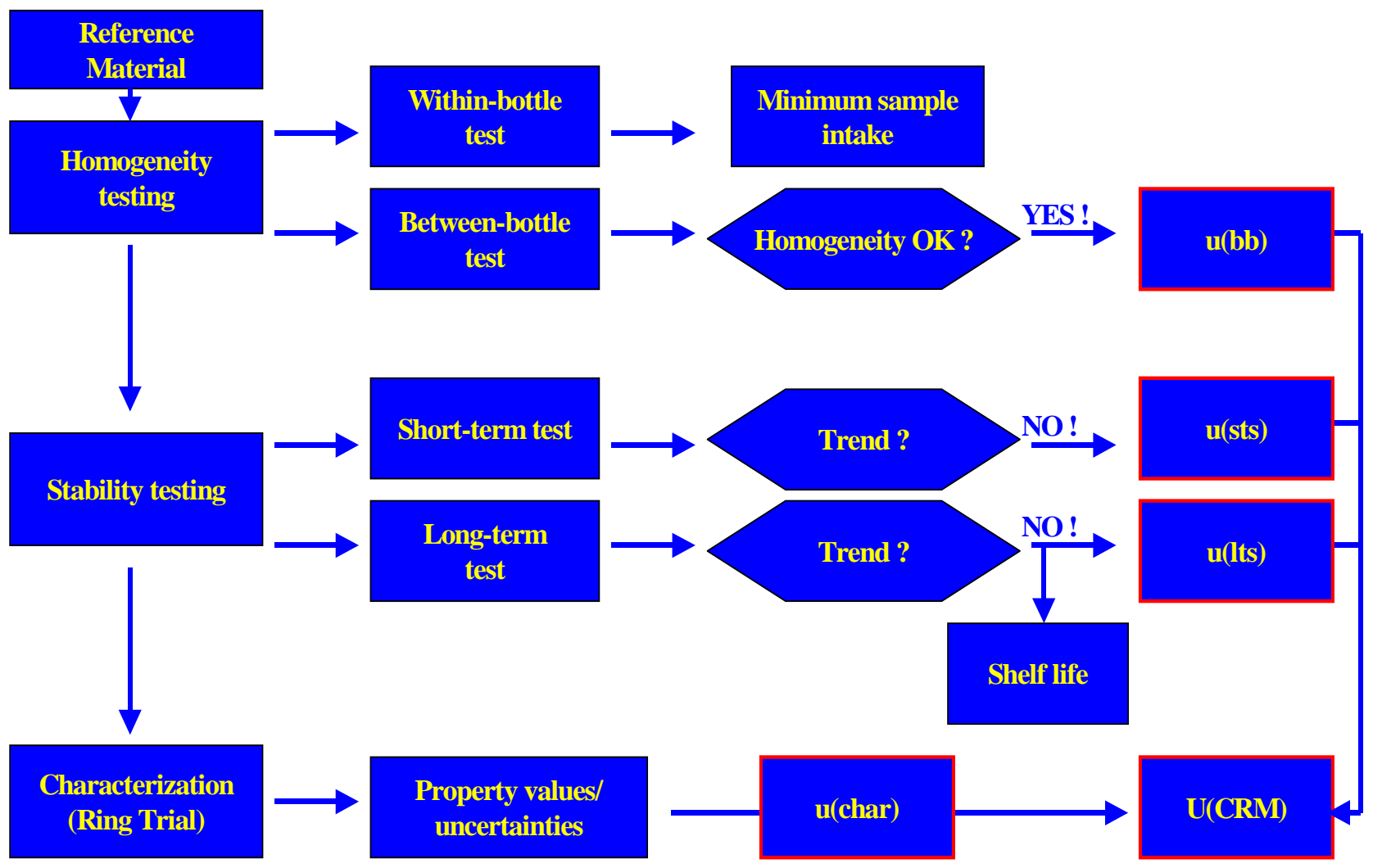

Further characterisation of the (C)RM is usually performed in a ring trial, where its fitness for purpose including the commutability can be evaluated with a rich repertoire of methodologies. Commutability is the property of a (C)RM that it must functionally behave the same as a real clinical specimen during the measurement by a routine procedure [19,36,37]. When a (C)RM is not commutable regarding one or some of routine measurement procedures, it might break the traceability chain and limits its applicability for quality control or proficiency testing. Therefore, the commutability testing of new (C)RMs against all routine measurement procedures in which it is expected to be used is essential. Obviously, the present definition of 'commutability' may not sufficiently address these aspects of qualitative analysis and new concepts are required.

Molecular genetic testing requires the highest level of data reliability based on proper QA of diagnostic laboratories as well as of their measurement and testing procedures. Thus, the need for appropriate reference materials which play a key role in the proof of method performance and proficiency testing became increasingly urgent. Aspects concerning the development, processing and expected characteristics of CRMs for genetic testing will be further outlined in the section 1.4. 


\subsubsection{Reference laboratories (reference measurement services)}

Reference laboratories are accredited analytical centres of excellence for the measurement of certain analytes that may collaborate in a network. These expert laboratories provide reference measurement services performing measurements with the greatestcompetence using reference measurement procedures and certified reference materials (CRMs). A further requirement for these laboratories is the participation in a specific collaborative survey (i.e. proficiency testing) under the auspices of metrological institutes (e.g. IRMM, NIST, etc.) or international organisations, such as the International Federation of Clinical Chemistry and Laboratory Medicine (IFCC) in laboratory medicine $[15,38]$.

Reference laboratories play an integral role in the implementation of measurement traceability through the assignment of target values with a reasonable low level of uncertainty to RMs (including calibrators and QC materials) $[38,39,40]$ and evaluating the analytical performance of measurement procedures $[38,39,41]$. The development and commutability testing of CRMs are carried out by inter-laboratory studies in a network of such referral laboratories [18]. In addition, reference laboratories act as consultants to the Commission, governments, industry, and organisations conducting external quality assessment schemes as well as to specialised individual laboratories. Requirements and specific aspects of reference laboratories are referred also in the European standard EN ISO 15195:2003 [42].

\subsubsection{External Quality Assurance (EQA) schemes}

External Quality Assurance schemes (also named proficiency testing, external quality control, or ring trial) are systems to verify laboratory competence for particular fields of testing. The principles of such programs are simple: an EQA scheme organizer distributes samples to participants for analysis, the participants report the results to the organizer for evaluation and get a feedback on their performance [21]. Today, EQA is the key mechanism to provide an objective external assessment of the overall performance of diagnostic medical laboratories and the efficiency of their analytical procedures (testing method, reagents, instrument and calibration). Therefore, EQA schemes are increasingly used for regulatory and post-market vigilance purposes in the EU as well as in many other countries. Ring trials also serve as educational tools and training for the participants to sustain improvements in the quality of services [21].

In addition, EQA schemes distributing proficiency testing materials with target values traceable to a RMS are able to detect standardisation, specificity and interference problems, as primary sources of inter-method and inter-laboratory performance data [21,41,43]. Furthermore, proficiency testing exercises are essential tools in the networking of reference laboratories [39,42], and can be used to evaluate the commutability of RMs [37,44]. These features of the EQA schemes are vital for the operation of RMSs. 
During the past years, both the numbers of molecular genetic EQA schemes available and the levels of participation have been increased significantly; however, the number of DNA targets remains limited to relatively few applications. EQA programs in molecular genetic testing are organised at different scales (national, regional, multinational, or at a large international level) and for different genetic disorders or risk factors [45]. Most of the schemes are mutation/disease specific; however, there are some technique-specific ring trials as well. The successive proficiency testing exercises have resulted in a gradual reduction in the rate of genotyping errors; however, for some types of molecular genetic testing still persist unacceptably high failure rates [46,47,48,VI].

At present, the lack of RMS in molecular genetic testing means that there are limitations in benchmarking against which a laboratory can judge the performance of its assays. Therefore, the development of CRMs and the recommendation of highly robust reference methods are needed in cases where the measurements are not reliable enough. On the other hand, current definitions of some essential metrological terms, such as the 'commutability' or the estimation and expression of uncertainty in measurements [49] do not cover the aspects of qualitative analysis sufficiently and new concepts are required.

\subsection{Regulation of genetic testing - Role of EU/EC with the IVD Directive}

In the EU, in vitro diagnostics, including genetic tests are regulated through the Directive 98/79/EC on in vitro diagnostic medical devices (IVD directive) [12] and its national transpositions (in Hungary, the order 8/2003. (III.13.) of the Ministry of Health and Social Affairs), which cover the commercial in vitro diagnostic tests. The main goal of the IVD directive is to introduce harmonised controls on the IVD medical devices (IVDs) throughout the EU. It is fully in force since December 2003 and deals with all aspects of safety and performance. Adherence to the directive regulations is mandatory and only IVDs bearing the CE-mark can be sold and used in the EU for human laboratory diagnostic purposes. For proof of compliance with the legal requirements, conformity must be demonstrated, when the manufacturer can refer to the harmonised European (EN) standards, which are technical documents for concretisation of legal requirements.

Furthermore, IVD manufacturers are obliged to assure the traceability of values assigned to their calibrators and control materials through available reference measurement procedures and/or available reference materials of a higher order [12 (Annex 1, section 3)]. The harmonised standard EN ISO 17511:2003 [15], which describes the importance of the metrological principles, takes an even stronger position on commitment to the cause of continuous improvement in reference methods and materials stating that: "It is the aim of metrology in laboratory medicine to improve metrological traceability ... by providing the missing reference measurement procedures and reference materials, based on international consensus.” These statements have particular relevance 
for molecular genetic tests, because the current lack of CRMs for molecular genetic tests leads to assay results that are not traceable to an accepted common standard.

Genetic tests are in vitro diagnostic tests and as such are covered by the legislation. All commercial kits that are sold for this purpose will carry the CE mark and come under the vigilance system of the governments of each country. These regulations do not cover reagents manufactured and used only within the same health institution (i.e. LDTs); however, there are some ambiguities in the interpretation of the directive concerning these 'in-house' reagents and assays, particularly where a laboratory is providing a testing service for other institutions as well as its own.

In addition, the Directive explicitly covers materials transferred between laboratories, which have particular relevance for molecular genetic tests: Laboratories need to validate any genetic test intended for patient testing by analyzing positive and negative samples for the genotype to be detected, in order to establish or verify sensitivity, specificity, and other performance characteristics of the test. For routine testing, well-characterised positive and negative samples need to be included in each run of patient specimens. For most genetic variants, such positive or negative specimens are not available commercially and the sole source of previously genotyped samples was the exchange between testing laboratories, which could be used also for external quality control purposes for variants not covered by EQA schemes. Thus, the demand for RMs for genetic testing is driven by a combination of regulatory requirements and the wish of testing laboratories to be assured that their results are correct.

\subsection{Reference materials for genetic testing}

Many of the parameters which may be critical for RMs in other areas (concentration, homogeneity, etc.) do not apply or are not so important in RMs for qualitative genetic tests. This is because most molecular genetic tests work efficiently over a range of input concentrations, and because the test will usually fail to give a result rather than giving an incorrect result if the input DNA concentration is outside this range [50]. On the other hand, several issues must be taken into consideration, which are specific in the development, preparation and certification of (C)RMs for genetic testing:

\subsubsection{Sources of DNA}

DNA can be synthesised or isolated from patients with characterised mutations or alleles known to cause the disorder of interest; however, the need of human specimen is closely linked to ethical questions and corresponding legal issues: Ethical approval and informed consent from the patient are prerequisites of the extraction of human DNA for genetic (C)RMs. Documentation and banking of the materials have to fulfil the legal requirements and an anonymisation of the material is needed as soon as possible in the chain. In case of common genetic variations, cell lines with confirmed genotypes can be the preferred source of control DNA [51,52]. 


\subsubsection{Validation methodologies}

In the absence of any reference or designated comparison method for genetic testing, reference materials must be certified by a consensus approach [26]. The range of methodological approaches is determined by the nature of the genetic change of interest. For example, large triplet repeat expansions (e.g. Fragile X syndrome, myotonic dystrophy) are not amenable to standard PCR amplification, and have to be analysed by Southern blotting or a modified PCR method, while SNPs are not readily detected by Southern blot, but can be identified by a wide range of other methods. In each case, the widest range of methods compatible with the particular RM should be applied in multiple replicates at different laboratories. Wherever possible, the RM has to be verified also by the direct determination of the nucleotide sequence, using multiple replicates in different centres. In addition, the interlaboratory variation in all parameters relating to testing equipment, environment, staff skills and training is immense. It will be vital, therefore, to test the fitness for purpose of RMs in as wide a variety of laboratories as possible in the frame of a ring trial [26].

\subsubsection{Formats of clinical genetic RMs}

There are many possible formats of genetic RMs, including CRMs: cell lines, genomic DNA (gDNA), recombinant DNA (rDNA), synthetic DNA (sDNA) or PCR products, all differing from typical clinical material for investigation. These possible types of molecular genetic RMs and their suitability under the headings discussed are tabulated below (Table 1.).

Table 1. Choosing a reference material formulation/presentation:

\begin{tabular}{|l|c|c|c|c|c|c|}
\hline \multicolumn{1}{|c|}{ Type } & $\begin{array}{c}\text { Similar to } \\
\text { usual samples }\end{array}$ & Versatile & Stable & $\begin{array}{c}\text { Economical } \\
\text { to produce }\end{array}$ & $\begin{array}{c}\text { Storage } \\
\text { Cost }\end{array}$ & $\begin{array}{c}\text { Ethical } \\
\text { issues }\end{array}$ \\
\hline Cell Line & +++ & +++ & - & + & - & + \\
\hline Genomic DNA & ++ & ++ & + & ++ & ++ & + \\
\hline Recombinant DNA & + & ++ & ++ & +++ & ++ & ++ \\
\hline PCR Product & + & ++ & ++ & +++ & ++ & ++ \\
\hline Synthetic DNA & - & - & ++ & ++ & ++ & +++ \\
\hline
\end{tabular}

Key: +++, excellent; ++, very favourable; +, moderate; -, less favourable

Every type has advantages and drawbacks and the choice of the right format mainly depends on the nature of the genetic alteration. Parameters to be considered are:

How similar is the reference material to the material usually received for testing?

Genetic testing laboratories extract DNA from various clinical specimens (e.g. blood, mouthwash, chorionic villi or indeed any tissue containing nucleated cells) by one of a large variety of methods. However, RMs cannot be prepared using a patient's sample directly, as sufficient material would not be available for a useful batch. Thus, the practical material most similar to the usual samples will be cultured cells or immortalised cell lines, which can be used also to assess the quality of 
DNA extraction procedures. As most testing requires purification of genomic DNA as a first step, this format must be the next most suitable material in terms of similarity to the usual samples; however, its purity from contaminants, salt concentration, average fragment size and many other parameters might be different from the DNA routinely tested in most testing laboratories, which can interfere with the proper functioning of tests in some laboratories. Finally, cell lines and gDNA contain also certain sequences, which are similar to the target sequence and may interfere with some genotyping methods not specific sufficiently. Recombinant DNA, PCR products and synthetic DNA fragments lack of such sequences as well as other genomic sequences, which are sometimes tested in the same reaction or used to control the amplification.

How versatile is the material? (i.e. Is it technically suitable for use in all the possible testing methodologies? - Is the material likely to be commutable?)

RMs based on short DNA fragments, whether cloned, produced by PCR or chemically synthesised will not be useful RMs for tests which involve Southern blotting, genome analysis platforms or long-range PCR, but they may be excellent RMs for short-range techniques, if the methods use primers inside of the fragment sequence. The testing methodologies employed are tailored to the type of mutation under study: short-range techniques for point mutations and long-range techniques for large triplet repeat expansions, and major gene re-arrangements. On the other hand, copy number of well characterised short DNA fragments can be more precisely determined and/or validated by independent techniques, which is crucial for quantitative assays as well as for the assessment of the stability and homogeneity of candidate RMs. However, short DNA fragments are better templates than genomic sequences, which results in a higher amplification efficacy. Thus, the optimal type of an RM will depend on what type of mutation the RM is designed for.

How stable is the material likely to be?

The stability of the RMs is of central importance and has to be guaranteed by the producer [34,53]. Although DNA is quite stable when stored under the appropriate conditions [50], optimal conditions have not been determined and very little has been published on the stability of different forms of DNA on long-term storage. Intact cells are likely to be the least stable of all the forms of RMs under consideration, as they need to be preserved at least at $-70{ }^{\circ} \mathrm{C}$ or preferably in liquid nitrogen to ensure their integrity. Stability characteristics of purified DNA depend on the purification method and on the storage conditions, but the optimal conditions need to be determined experimentally.

How expensive or economical is it to produce large batches of the material?

In order to meet the requirements of a certified reference material, the material will need to be capable of being prepared in large batches suitable for many hundreds or even thousands of tests. 
This is possible for all of the RM types envisaged, but is likely to be much more economical in the cases of PCR products and cloned DNA fragments.

How expensive or economical is it to store and to transport the material?

The parameters contributing to the cost of storage and transport are mainly stability (discussed above) and hazard. Storage and transport costs will of course rise sharply with decreasing stability because of the requirement for refrigeration/freezing. Hazards associated with biological materials include infectious agents which may be harboured by cell lines, but are unlikely to be present in purified DNA.

Are there ethical or ownership issues associated with the material?

Apart from synthetic DNA, which is the least suitable material from other points of view, all types of RM are derived directly or indirectly from a human source, which might lead to ethical concerns and privacy issues. Furthermore, materials derived from human sources may be the subject of ownership claims when used for commercial purposes. The likelihood of such problems is decreasing, the further removed from a human source an RM is. Cloned DNA fragments are removed from their genomic context, but their derivation must be documented under Genetically Modified Organism (GMO) regulations. PCR products must be derived from a genomic DNA sample, which is traceable to an individual.

\subsubsection{Intellectual property right (IPR) issues}

The EU Directive 98/44/EC [54] aiming to harmonise national patent laws of the Member States concerning biotechnological inventions has given mandate to issue patents on isolated genes and nucleotide sequences. Although an absolute product protection for the isolated DNA molecules as such was seriously debated, both the European Patent Office and the United States Patent and Trademark Office granted patents for diagnostic or therapeutic use of gene sequences, covering any method of diagnosis a disease or predisposition for it using the normal sequence of the respective gene $[55,56]$.

Patents on human DNA sequences have strong influence on the genetic diagnostics and partly also on research and development. Such gene patents affect also the installation of appropriate reference measurement systems, because they may arrest the use of reference measurement procedures, the development and use of CRMs and the whole standardisation and harmonisation process of patented genes' testing [7,56].

\subsubsection{Priorities in the development of CRMs for molecular genetic testing}

There are thousands of different molecular genetic tests, each in principle requiring a separate RM, and novel tests become possible all the time due to the research efforts to discover new SNPs and elucidate their role in disease, predisposition and diagnosis. 
This rapid evolution of molecular genetic testing exceeds the capacity to generate RMs and CRMs, which cannot immediately cover all diseases, given the time and high cost of developing them. As an initial approach, ranking-criteria are needed to set for prioritising CRM to be developed. Key preferences may be e.g. (i) the number of tests performed annually and availability of commercial genetic testing kits; (ii) the frequency of the allelic variants; (iii) frequency and impact of an inaccurate test result, (iv) existing tools for quality assurance (alternative techniques, e.g. functional assays), (v) proven clinical significance, clear indications and therapeutic consequences, etc. Additionally, commercial or governmental funding may facilitate the development of (C)RMs and many QC RMs are developed for EQA schemes.

Diseases and risk factors selected as target sequences for the development of prototype RMs or candidate CRMs correspond to these general rules.

1. Hereditary haemochromatosis $(\mathrm{HH})$ [57] is an autosomal recessive disorder of iron metabolism. The C282Y and the H63D mutations in the HFE gene on chromosome 6 are the two most common mutations associated with $\mathrm{HH}$. The C282Y mutation arises from a single base pair substitution (G>A) at nucleotide 845, resulting in an amino acid change of cysteine to tyrosine at codon 282. The H63D mutation arises from a single base pair substitution $(\mathrm{C}>\mathrm{A})$ at nucleotide 187 , resulting in an amino acid change of histidine to aspartate at codon 63.

2. Cystic fibrosis (CF) [58] (or mucoviscidosis) is an autosomal recessive disorder caused by a functional mutation in the gene of the cystic fibrosis transmembrane conductance regulator (CFTR) protein. CFTR functions as a chloride channel and controls the regulation of other transport pathways. Cystic fibrosis is a serious life-shortening disease hallmarked by abnormal mucus viscosity, which leads to progressive damage of the respiratory system, chronic problems in the digestive tract as well as to chronic infections. Although nearly 1500 different CF alleles have been identified, 70\% of the CF population carries the most common $\Delta$ F508 mutation.

3. Fragile $\mathrm{X}$ syndrome [59] is an X-linked genetic disorder caused by a mutation in the FMR1 gene known as trinucleotide repeat expansion, which is characterised by moderate mental retardation in affected males and mild mental retardation in affected females. Average number of these (CGG) trinucleotide repeats is around 30 (range 6-50) in most individuals. In people with the fragile X syndrome, the FMR1 allele has over 200 CGG repeats. Such degree of expansion of CGG repeats results in methylation of that part of the DNA, which leads to transcriptional silencing of the FMR1 protein. The range of 59-200 repeats is generally referred to as premutation, which is unstable and has a high risk of expanding to a full mutation. Although premutation carriers are usually not affected by 
the fragile $\mathrm{X}$ syndrome, they carry the disorder and can transmit it to their children. Intermediate or "gray zone" alleles (50-58 repeats) represent the fourth state, which may or may not be unstable. Determination of the number of CGG repeats and their methylation status might be carried out using Southern blot analysis and methylation-sensitive restriction endonuclease digestion.

4. Leiden mutation [60] of the human coagulation Factor V (FV) is a G to A substitution at nucleotide position 1691, which results in the arginine at position 506 being replaced by a glutamine. Normally, activated Protein C (APC) inactivates FVa by an initial hydrolysis of the peptide bond at the carboxyl group of the arginine 506 followed by a cleavage at the arginine 306. The disruption of the primary cleavage site of FV by the Leiden mutation results in a resistance to the anticoagulant activity of APC. FV Leiden is the most common inherited risk factor for venous thromboembolism, present in $5 \%$ of the general population but is found in $20 \%$ of venous thromboembolism (VTE) cases.

5. G20210A mutation in the 3' untranslated region of the coagulation Factor II (prothrombin) gene was discovered in 1996 by Poort et al. [61] and leads to significantly higher mean plasma prothrombin levels and increased risk for VTE. The heterozygous mutation occurs with a frequency of approximately $2-5 \%$ in the Caucasian population. The clinical significance of this SNP, the indications for testing and also the therapeutic consequences have been elucidated [62], and the analysis of this SNP is one of the most frequently applied genetic tests in clinical laboratories, because no specific functional (e.g. clotting) tests exist.

\subsection{Initiatives for the development of (Certified) Reference materials for genetic testing}

The IPTS-ESTO report [7] provided objective information about the fragmentation and lack of standardisation and harmonisation in the field of genetic testing in the EU and described the need for substantial improvement of the quality of all aspects of the services, based on internationally established standards. In response to these shortcomings and controversies, the stakeholders of this field gave rise to several initiatives in order to improve quality, harmonisation and standardisation. The European Commission, Joint Research Centre, Institute of Reference Materials and Measurements (IRMM) joined to several of these initiatives and launched novel projects to develop (C)RMs for genetic testing as well as to improve the overall quality of testing services in the field.

\subsubsection{The Certified Reference Materials for Molecular Genetic Testing (CRMGEN) project}

The first major initiative, the Certified Reference Materials for Molecular Genetic Testing (CRMGEN) project started in November 2001. The FP5 CRMGEN [7] project focused on the development of candidate RMs for molecular genetic testing of inherited disorders, such as hereditary haemochromatosis (HH), fragile X syndrome (FraX) Duchenne muscular dystrophy (DMD), and 
cystic fibrosis (CF). In the frame of this shared cost action (SCA) project, different formats of clinical genetic RMs have been developed, produced and investigated for their suitability for RMs. Four basic types of molecular genetic RMs were examined: cultured cell lines, recombinant DNA (plasmids), enzymatically amplified DNA fragments (PCR products) and genomic DNA. The prototype RMs developed were characterised and subjected to extensive field trials in diagnostic molecular genetic testing laboratories across Europe. The knowledge gained in this feasibility study has been utilised in the development and production of (C)RMs for genetic testing [26].

\subsubsection{Development of CRMs for the identification of the human Factor II G20210A variant and} related projects

In line with the FP5 CRMGEN SCA, the Scientific Committee of Molecular Biology Techniques in Clinical Chemistry (C-MBT) of the IFCC has initiated a joint project with the IRMM to develop and produce a set of plasmidic CRMs for the analysis of the human coagulation Factor II (prothrombin) gene G20210A variant [I,II,III,IV,V]. The reference plasmids produced in this project were used also in an 'exploratory research project' “Feasibility study: Preservation of plasmidic DNA" to optimise the preservation of DNA- and particularly plasmid-based RMs.

Furthermore, the IRMM and the Department of Biochemistry of the University of Szeged agreed on a proficiency testing (PT) study in cooperation with 3 European external quality assessment (EQA) organisations [VI]. In the frame of this study, new mutations were introduced into the wild-type (wt) FII reference plasmid by site-directed mutagenesis and the QCMs containing the modified and/or reference plasmids were distributed to the participants to assess how clinical laboratories interpret unusual genotyping results and how many of these laboratories recognize interfering mutations and report them correctly. Additional aims of the study were to test identify weaknesses of genetic testing services, to develop QCMs for rare variants, and to gather more information about the requirements for DNA-based candidate RMs.

\subsubsection{The EuroGenTest Network of Excellence project}

The FP6 project EuroGenTest Network of Excellence (NoE) [63] intended to organize, harmonize and improve the overall quality of genetic testing services dealing with several further aspects of the field including legal, health policies and health economic impact, intellectual property right issues, ethical and social questions: confidentiality, informed consent, employment and insurance, etc. Concerning the analytical quality of the services, the FP6 EuroGenTest NoE worked on amelioration of the structure and harmonisation of EQA schemes, facilitated the development of guidelines and supported the development and validation of novel techniques as well as the accreditation/certification of the genetic testing services. 


\section{AIMS AND SCOPE}

The ultimate goals of this thesis were the development and production of DNA-based (C)RMs for clinical molecular genetic testing and to contribute to the quality improvement in genetic testing services.

In order to achieve these general objectives, the main aims of the present study were:

1) To process different types and presentation formats of DNA-based RMs, and to investigate their suitability for clinical genetic (C)RMs.

2) To optimize the preservation of DNA-, in particular plasmid-based RMs.

3) We aimed to elaborate the know-how for the development and production of (C)RMs for any genetic tests, including the optimisation of study protocols for the assessment of the stability and homogeneity of genetic RMs as well as to establish a model system for certification of DNA-based RMs.

4) We aimed to develop and produce a set of plasmidic CRMs for the quality assurance of PCRbased methods used for the detection of the G20210A variant in the human coagulation Factor II gene.

5) We intended to design, organize and carry out commutability studies on these candidate CRMs to demonstrate their fitness for purpose.

6) Utilizing the wild-type and mutant Factor II reference plasmids and the site-directed mutagenesis technique, we pursued to develop QCMs for rare SNPs.

7) We aimed to carry out a European proficiency testing exercise using the newly developed Factor II QCMs for the detection of G20210A and rare SNPs to identify the error sources in SNP genotyping as well as to assess the competence of clinical laboratories to recognize interfering sequence variants resulting in unusual genotyping results. 


\section{MATERIALS AND METHODS}

\subsection{Origin and Production of RMs}

\subsubsection{Origin of the CRMGEN prototype RMs}

In the frame of the CRMGEN project, four basic types of prototype RMs were processed and investigated as pilots for their stability and homogeneity: PCR products for hereditary haemochromatosis (CRMGEN-HH), genomic DNA for fragile X syndrome (CRMGEN-FraX), plasmid for the Leiden (G1691A) mutation in the coagulation Factor V (CRMGEN-FV) gene and cell pellets for cystic fibrosis (CRMGEN-CF). The development of these RMs was performed in clinical genetic centres in parallel with the development of RMs for other inherited disorders (e.g. Duchenne muscular dystrophy, Sickle cell anaemia, etc.). The study was carried out according to the local, national and European regulations and source materials were collected under informed consent from the patients. Lymphocytes or genomic DNA extracted from venous blood were anonymised. The prototype RMs specified below were designed and processed in close cooperation with the IRMM.

\section{CRMGEN-HH - PCR product based prototype RMs}

The prototype PCR product RMs for the analysis of the human HFE SNPs C282Y and H63D were developed in the National Centre for Medical Genetics, Our Lady’s Hospital for Sick Children (Crumlin, Ireland) using a long range nested PCR technique with Pfu DNA polymerase. The first PCR generated a 3353 bp master template (GenBank accession number Z92910, nucleotide position 4307 to 7660), which was diluted $1 / 10^{4}$ in Tris-EDTA (TE) buffer. The 2878 bp RM sequence (nt 4333 to 7211) was generated in the second round of PCR. This RM sequence encompasses both the C282Y (G6722A) and the H63D (C4762G) mutations (Figure 5.).

Figure 5. Schematic representation of $\mathrm{HH}$ mutations in HFE gene and primer positions (arrows) for development of CRMGEN-HH RMs.

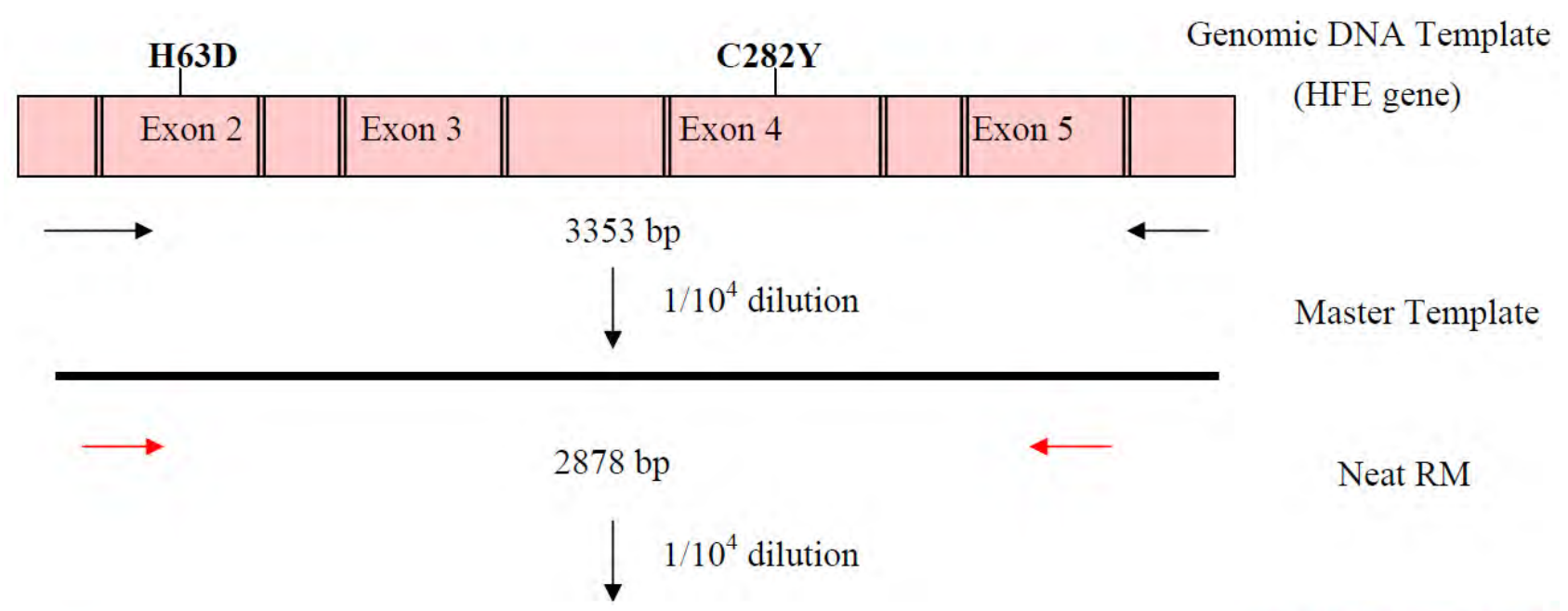


The PCR product RMs generated were diluted again $1 / 10^{4}$ in TE buffer without or with $100 \mu \mathrm{g} / \mathrm{ml}$ sonicated salmon sperm DNA (SS DNA) as carrier. Stocks of this prototype RMs were aliquoted into sterile, self standing, $1.5 \mathrm{ml}$ high recovery polypropylene tubes (Axygen, Ref: SCT-150-L-C-S, SS) at $100 \mu \mathrm{l} /$ vial and half of these samples were freeze-dried (FD) in a CHRIST LPC-16 EPS 2-65DS freeze-dryer at the IRMM. Finally, to examine different approaches for stabilisation of purified DNA, 8 CRMGEN-HH prototype RMs (genotype C282Y/H63D normal or compound heterozygous, with and without carrier SS DNA, freeze-dried (FD) or not (NFD) were produced.

CRMGEN-CF - Cell line based prototype RMs

Epstein-Barr virus (EBV) transformed lymphocytes were generated from blood that was obtained from individuals (CF patients and/or CF carriers) carrying CFTR mutations ( $\Delta \mathrm{F} 508 / \Delta \mathrm{F} 508$;

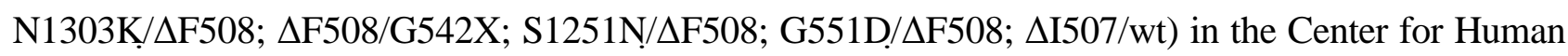
Genetics, Katholieke Universiteit Leuven (Leuven, Belgium). These immortalised CF cell lines were sent to the National Institute for Biological Standards and Control (NIBSC, Hertfordshire, UK) for large scale culturing. Cell lines were characterised, validated and checked for their integrity and tested for sterility including Mycoplasma testing. The cells were stored in liquid nitrogen and two cell-line based reference materials were transported to the IRMM for long-term backup storage.

CRMGEN-FraX - genomic DNA based prototype RMs

EBV immortalised cell lines have been established from 13 Fragile-X normal, premutation and full mutation individuals in the National Genetics Reference Laboratory (NGRL) Manchester (Manchester, UK) and transferred to the NIBSC for large scale culturing, testing and characterisation. Confirmation of the expected genotypes and assessment of the stability of the triplet repeats were carried out in Leuven and Manchester. The FMR1 alleles were genotyped in both the blood and cell line samples using PCR/PAGE Genescan analysis followed by Southern blotting to determine the methylation status of region surrounding the CGG repeat. Whole genomic DNA was extracted from a stable lymphoblastoid cell line using four different extraction methods, such as two different phenol/chloroform extraction protocols, a sodium perchlorate/chloroform and the Puregene 'salting out' methodology. Extracted gDNA was homogenised by constant mixing at different temperatures and forcing through increasingly smaller needles and diluted to $150 \pm 10 \mathrm{ng} / \mu \mathrm{l}$ concentration in TE buffer. The materials were aliquoted into sterile, self standing $1.5 \mathrm{ml}$ high recovery tubes (ca. $5 \mu \mathrm{g}$ DNA in a volume of $33 \mu \mathrm{l}$ ). The prototype RMs for testing different DNA extraction and stabilisation techniques were prepared using a cell line generated from a normal heterozygous female (Allele 1: (CGG) $)_{10}$, AGG, (CGG) ${ }_{11}$; Allele 2: (CGG) $)_{10}$, AGG, (CGG) , AGG, (CGG) $)_{10}$; except of the repeat region, FMR1 sequences corresponded to the GenBank accession No.: M67468). Half of the vials processed from each CRMGEN-FraX prototype RM, were lyophilised at the IRMM using a CHRIST LPC-16 EPS 2-65DS freeze-dryer. 


\section{CRMGEN-FV - Plasmidic prototype RMs}

The human coagulation Factor V (proaccelerin, FV) sequences were isolated from genomic DNAs extracted from venous blood, which was collected from a thrombophilic patient (homozygous for the "Leiden” mutation) and a healthy individual (homozygous wild-type) at the Leiden University Medical Center, (LUMC; Leiden, The Netherlands). DNA fragments containing the G1691A "Leiden” mutation or the wild-type (G1691) sequence were cloned through SacI/BamHI digestion in pUC19 vector at the Department of Human and Clinical Genetics. Except of the "Leiden" mutation, the 585 bp insert corresponded to the exon 10 sequence of Factor V gene (nt 3829638880, GenBank accession number: L09137; “Leiden” mutation: 38592G>A). Following amplification and purification according to the protocol described earlier [I], these plasmids were used for the production of the CRMGEN-FV prototype RMs mimicking the heterozygous genotype. The first series of CRMGEN-FV prototype RMs were dissolved in TE buffer solution at $\mathrm{pH} 7.5$ containing $2 \times 5 \times 10^{6}$ copies/ $\mu \mathrm{l}$ in $50 \mu \mathrm{l} /$ vial without additive or with different additives (10\% sucrose / 50\% glycerol / salmon sperm DNA crosslinked with a Psoralen/UVA treatment). The $2^{\text {nd }}$ batch of CRMGEN-FV prototype RMs were prepared in a 10-times concentrated TE buffer (10 mM : $1 \mathrm{mM}$, $\mathrm{pH}$ 8.0) containing $2 \times 5 \times 10^{6}$ plasmids/ $\mu \mathrm{l}$ in $50 \mu \mathrm{l} /$ vial. The second series consisted of 3 prototype RMs: without additive FD / not FD and with 10\% of sucrose FD. All CRMGEN-FV prototype RMs were filled in sterile, self-standing, screw cap high recovery polypropylene tubes. Lyophilisation was carried out at the IRMM using a CHRIST LPC-16 EPS 2-65DS freeze-dryer.

\subsubsection{Origin of the NIBSC-WHO candidate Reference Panel samples}

The National Institute for Biological Standards and Control (NIBSC) in cooperation with the World Health Organization (WHO) developed gDNA based reference materials for the Factor V Leiden Mutation and for Prothrombin Mutation G20210A. DNA samples for these sets of RMs were extracted using a 'salting out' method and suspended in TE buffer with $5 \mathrm{mg} / \mathrm{mL}$ Trehalose as excipient before freeze-drying. The candidate NIBSC-WHO Reference Panel for the Factor V Leiden Mutation [64] was subjected to a collaborative study (CS 246) [65] in 2004 and the candidate Reference Panel for Prothrombin Mutation G20210A [66] was tested in a similar field trial (CS 262) [67] beginning of 2005. IRMM was asked to participate in both of these NIBSCWHO collaborative studies. In each study, three sets of 6 coded samples had to be genotyped in each study on three separate days using the routine genotyping tests.

\subsubsection{Preparation of prototype RMs for the feasibility study to optimise DNA preservation}

The insufficient stability of the $1^{\text {st }}$ batch of the candidate prothrombin CRMs triggered an 'exploratory research project' on the preservation of DNA- and particularly plasmid-based RMs. In the frame of this systematic study, several prototype RMs were prepared using the wild-type FII 
reference plasmids at $1 \mathrm{ng} / 50 \mu \mathrm{L}$ with different additives listed in the Table 2 . freeze-dried or not. EDTA [Cat\# E-5134], Tris [Fluka, Cat\# 93350] and Tris-HCl [Cat\# E-2788] were purchased from Sigma-Aldrich, $\mathrm{NaOH}$ [Cat\# 1.06482.1000] used for $\mathrm{pH}$ adjustment was ordered from Merck.

These series of prototype FII RMs were tested after 12 weeks of storage.

Table 2. Additives used for the preparation of prototype FII G20210 RMs in the feasibility study

Additive(s) dissolved in Tris-EDTA buffer (1 mM : $0.1 \mathrm{mM})$, pH 8.0

No additive (Tris-EDTA buffer (1 mM : $0.1 \mathrm{mM}$, pH 8.0)

Tris-EDTA buffer (10 mM : $1 \mathrm{mM}, \mathrm{pH} 8.0)$

$1 \mathrm{mg} / \mathrm{mL}$ bovine serum albumin (BSA) [Sigma, Cat\# B-6917]

5\%, 10\%, 20\% of Sucrose [Merck, Cat\# 1.07687]

0.5\%, 5\%, 10\%, 20\% of D(+)Trehalose [Sigma, Cat\# T-0167]

$50 \%$ of Glycerol [Sigma, Cat\# G-7757]

$5 \%$ of D-Fructose [Merck, Cat\# 4007]

5\% of D-Mannitol [Merck, Cat\# 5987]

$5 \%$ of D-Sorbitol [Sigma, Cat\# S-3889]

1\% Tween 20 [Sigma, Cat\# P-9416]

1\% of PEG 8000 [Sigma, Cat\# P-5413]

$1 \%$ of Na-L(+)ascorbate [Merck, Cat\# 5.00076]

$0.01 \%$ of SDS [Bio-Rad (10\% solution) Cat\# 161-0416]

$200 \mu \mathrm{mol} / \mathrm{L}$ Diethylene Triamine Pentaacetic Acid (DTPA) [Merck, Cat\# 1.08426.0100]

$200 \mu \mathrm{mol} / \mathrm{L}$ desferrioxamine [Novartis, Cat\# 484075]

3.1.4. Production of the candidate CRMs IRMM/IFCC-490, -491 and -492 and the proficiency testing samples for the analysis of the prothrombin G20210A variant

Origin of extracted DNA and production of the $1^{\text {st }}$ batch of the candidate CRMs for the analysis of the G20210A variant in the human coagulation Factor II gene had been reported earlier [I]. Preparation of the $2^{\text {nd }}$ batch was published [V] and described in details in the certification reports [II,III,IV]. Development and processing of the external quality control samples have been published [VI] as well. Briefly, the prothrombin gene fragment was selected in the Molecular Biology Division, Department of Clinical Chemistry and Laboratory Medicine, Medical University Vienna (Vienna, Austria). This 609 bp fragment chosen (GenBank accession number M17262, nt 26302 to 26910) spans all primer annealing sites published until today. After initial expansion and characterisation in a PCR2.1 vector, the constructs were sent to the IRMM, where the wild-type and the G20210A mutant FII gene inserts were subcloned into the non-proprietary pUC18 plasmid. The C20209T mutation was introduced into the wild-type sequence using the site-directed mutagenesis technique in the Department of Biochemistry, Faculty of Medicine, University of Szeged (Szeged, Hungary). 
The first batches of the candidate CRMs were processed as lyophilised material containing $5 \times 10^{4}$ copies of plasmids in $100 \mu \mathrm{l}$ solution and $100 \mu \mathrm{g} / \mathrm{ml}$ bovine serum albumin (BSA) as additive. The second batches of the candidate CRMs and the QCMs were prepared in TE buffer (10 mM Tris : 1 mM EDTA, pH 8.0) at a concentration of ca. 20 and 10 pg/ $\mu \mathrm{L}$ DNA, respectively.

\subsubsection{Freeze-drying protocol developed for the lyophilisation of DNA-based RMs}

DNA-based RMs were lyophilised using a CHRIST LPC-16 EPS 2-65DS freeze-dryer following the protocol described in the Table 3. below:

Table 3. Lyophilisation protocol developed for the free-drying of DNA-based RMs

\begin{tabular}{llcccc}
\hline Section & Phase & Time $(\mathrm{h}: \mathrm{m})$ & Temp. $\left({ }^{\circ} \mathrm{C}\right)$ & Vacuum (mBar) & Saf.press. (mBar) \\
\hline 1 & Start values & $-:-$ & -40 & OFF & OFF \\
2 & Freezing & $01: 00$ & -40 & OFF & OFF \\
3 & Freezing & $02: 00$ & -40 & OFF & OFF \\
4 & Preparation & $01: 00$ & -40 & OFF & OFF \\
5 & Sublimation & $03: 00$ & -40 & 0.080 & 0.120 \\
6 & Sublimation & $03: 00$ & -35 & 0.080 & 0.200 \\
7 & Second. Drying & $03: 00$ & -30 & 0.080 & 0.200 \\
8 & Second. Drying & $03: 00$ & -25 & 0.080 & 0.200 \\
9 & Second. Drying & $06: 00$ & +30 & 0.080 & 0.200 \\
\hline
\end{tabular}

\subsection{Assessment of stability and homogeneity of RMs}

\subsubsection{Assessment of stability of RMs}

According to the ISO Guides 34 and 35 [31,34], both the prototype and the candidate RMs were subjected to stability studies following an isochronous scheme developed at the IRMM [35] and presented on the Figure 6.

The protocol consisted of a short- and long-term isochronous study for every RM. Samples for the short-time stability studies were stored for 2,4 and 8 weeks at $40^{\circ} \mathrm{C}, 18^{\circ} \mathrm{C}, 4^{\circ} \mathrm{C}$ and $-20^{\circ} \mathrm{C}$; however, the CRMGEN-HH prototype RMs were stored at $60^{\circ} \mathrm{C}$ instead of $40{ }^{\circ} \mathrm{C}$. Long-term stability of RMs were investigated by storing the RMs at $18{ }^{\circ} \mathrm{C}, 4^{\circ} \mathrm{C}$ and $-20{ }^{\circ} \mathrm{C}$ for 4 , 8, and 12 months. However, long-term stability the IRMM/IFCC candidate FII CRMs was tested in 6, 12 and 24-month stability studies. In both type of stability studies, the safety factor was 2 and the reference temperature was set at $-70^{\circ} \mathrm{C}$, corresponding to time point 0 . In order to reduce the uncertainty related to the measurement results, three units per time point were tested in 4 replicates using 4 dilutions (i.e. 1, 1:10, 1:100, and 1:1000) under repeatability conditions. Selection of samples for the stability studies was randomised from the whole batch of a RM. After analysis, the data were screened for suspicious values and a regression line was calculated for evaluation of statistically and technically significant trends ( $t$-test; $t 0.05,22$ ). A one-way analysis of variance (ANOVA) was used to estimate the uncertainty related to the stability of the RMs at least for the planned shelf-life [68]. 
Figure 6. Scheme of the isochronous stability studies (short-term)

Reference Temperature: $-70^{\circ} \mathrm{C}$
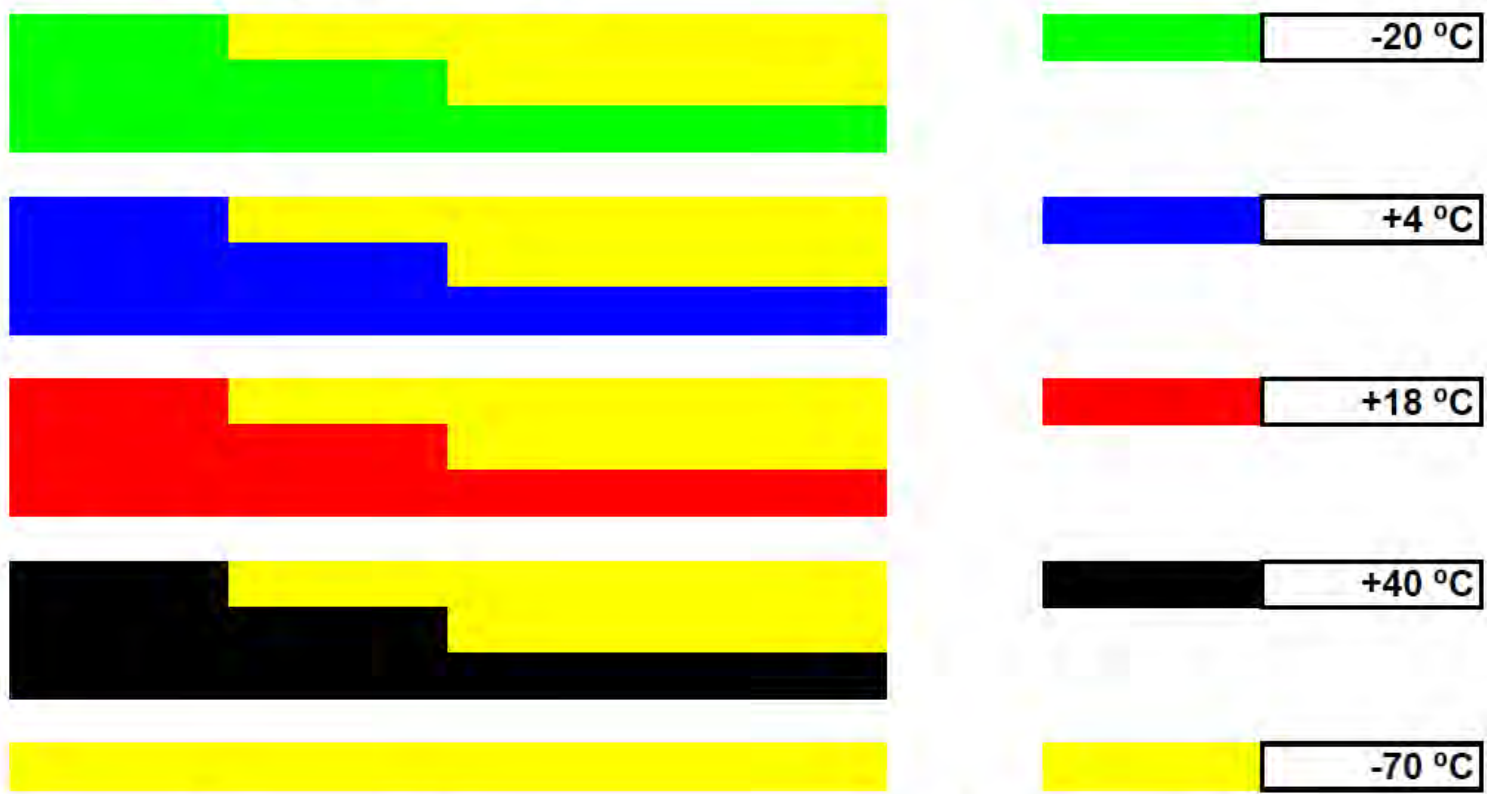

2

4

8 weeks

\subsubsection{Assessment of homogeneity of RMs}

Homogeneity of RMs could be deduced from the samples stored at $-70{ }^{\circ} \mathrm{C}$ and at $-20{ }^{\circ} \mathrm{C}$ (i.e. the usual storage temperature of the genetic RMs tested, $n=18$ ) in the short-term stability study. Between-bottle and within-bottle homogeneity of the candidate CRMs and some of the prototype RMs (e.g. CRMGEN-FV RMs) was assessed on the basis of the Cp values using the software Microsoft Office Excel single factor ANOVA (Microsoft Corporation, Silicon Valley Campus, CA, USA) [69]. Most of the prototype RMs were tested in 4 replicates at 4 dilutions (i.e. 1, 1:10, 1:100, and 1:1000) using qualitative PCR methods, which was sufficient for the feasibility study.

\subsubsection{Assessment of the freeze-thaw tolerance of prototype RMs}

Liquid plasmidic prototype RMs containing various additives (see 2.1.3.) were tested for any degradation after 10 freeze-thaw cycles within 6 days. Samples stored at $+4{ }^{\circ} \mathrm{C}$ served as control.

\subsection{Genotyping techniques}

\subsubsection{Sample preparation and DNA quantitation by PicoGreen}

Lyophilised RM samples were reconstituted in the required amount of nuclease-free water (Promega), which was used also for the preparation of the different dilution series.

DNA concentrations were determined using the PicoGreen double-stranded DNA quantitation kit (Molecular Probes), following the manufacturer's instructions. Copy number of target sequences in the stock solutions was calculated on the basis of the DNA concentration and the size of the DNA, assuming that $1 \mathrm{pg}$ of double-stranded DNA corresponds to $979 \mathrm{Mbp}$. 


\subsubsection{Analysis of the C282Y and H63D mutations in the human HFE gene}

Multiplex PCR-RFLP method: CRMGEN-HH prototype RMs were tested according to the method described by Stott et al. [70]. This multiplex PCR method amplifies the C282Y and H63D loci of the human HFE gene simultaneously in $25 \mu \mathrm{l}$ reaction volume using mutagenic primers (282-mut 5'-gTA CCC CCT ggg gAA gAg CAg AgA TAC A-3'; 282-rev 5'-CCA TCC CCT AAC AAA gAg CAg ATC CAC-3'; 63-fwd 5'-CAC ACT CTC TgC ACT ACC TCT T-3'; 63-mut 5'-ggC TCC ACA Cgg CgA CTC ACg T-3' ordered from Proligo (http://www.proligo.com/). The sample intake used was $1 \mu$ l. Nuclease free water was served also as no template control. Platinum Taq DNA Polymerase (the kit contains also 10× buffer and $50 \mathrm{mM}$ magnesium chloride), $100 \mathrm{mM}$ dNTP set, PCR grade and 100 bp DNA ladder were ordered from Invitrogen Life Technologies. The reaction was carried out using a GeneAmp 9700 PCR thermal cycler from Applied Biosystems (Applera Belgium). Following the overnight digestion with BbrPI restriction endonuclease (Roche Applied Science), $15 \mu$ l of PCR products were mixed with $1.5 \mu$ l of $10 \times$ Loading Buffer (Invitrogen) and run on a 2.5 \% ultrapure electrophoresis grade agarose gel (Gibco BRL, Invitrogen) in a Tris-Borate buffer containing $0.5 \mathrm{mg} / \mathrm{ml}$ ethidium bromide, at $150 \mathrm{~V} / 150 \mathrm{~mA}$ for $30 \mathrm{~min} .5 \mu \mathrm{l}$ of $100 \mathrm{bp}$ ladder and $5 \mu \mathrm{l}$ of undigested PCR product DNA were loaded as control. The gels were then visualised on a transilluminator and photographed using the GeneSnap program (SynGene, Westburg).

Multiplex real-time PCR method: In order to compare the copy number of target sequences more precisely in the various CRMGEN-HH prototype RMs after the stability studies, a multiplex realtime PCR method was adapted from the literature [71]. This method applies the LightCycler (LC) instrument (Roche Applied Science) with the fluorescent resonance energy transfer (FRET) technique for genotyping the $\mathrm{C} 282 \mathrm{Y}$ and H63D mutations in the human HFE gene. However, the original procedure was slightly modified to get clearer melting curves as well as to enable a relative quantitation of the samples tested.

PCR was performed in disposable LC capillaries (Roche Applied Science) using a reaction volume of $20 \mu \mathrm{L}$, containing $1 \mu \mathrm{L}$ of DNA (i.e. the respective dilution of the a CRMGEN-HH prototype RM), 0.2 mM each of the primers (C282Y-fw: 5'-Tgg CAA ggg TAA ACA gAT CC-3'; C282Yrev: 5'-TAC CTC CTC Agg CAC TCC TC-3'; H63D-fw: 5'-CAC ATg gTT AAg gCC TgT Tg-3'; H63D-rev: 5'-gAT CCC ACC CTT TCA gAC TC-3') $2 \mu \mathrm{L}$ of reaction buffer (LightCycler FastStart DNA Master Hybridization Probes 10× buffer; (Roche Applied Science), $0.8 \mu \mathrm{L}$ of $25 \mathrm{mM} \mathrm{MgCl} 2$ stock solution, and $0.1 \mathrm{mM}$ each of the probes (C282Y-det: 5'-AgA TAT ACg TAC CAg gTg gAg3'-Fluorescein; C282Y-anch: LCRed640-5'-CCC Agg CCT ggA TCA gCC CCT CAT TgT gAT CTg gg-3'-P; H63D-det: 5'-ACg gCg ACT CTC ATC ATC ATA gA-3'-Fluorescein; H63D-anch: LCRed705-5'-CAC gAA CAg CTg gTC ATC CAC gTA gCC CAA AgC TTC AA-3'-P). 
The PCR conditions were as follows: initial denaturation at $95{ }^{\circ} \mathrm{C}$ for $10 \mathrm{~min}$, followed by 40 cycles of denaturation $\left(95^{\circ} \mathrm{C}\right.$ for $\left.0 \mathrm{~s}, 20^{\circ} \mathrm{C} / \mathrm{s}\right)$, annealing $\left(58^{\circ} \mathrm{C}\right.$ for $\left.10 \mathrm{~s}, 20^{\circ} \mathrm{C} / \mathrm{s}\right)$, and extension $\left(72^{\circ} \mathrm{C}\right.$ for $12 \mathrm{~s}, 20^{\circ} \mathrm{C} / \mathrm{s}$ ). The melting curve analysis consisted of 1 cycle at $95^{\circ} \mathrm{C}$ for $10 \mathrm{~s}$ and $45^{\circ} \mathrm{C}$ for $5 \mathrm{~min}$, followed by an increase of the temperature to $80^{\circ} \mathrm{C}$ at $0.1^{\circ} \mathrm{C} / \mathrm{s}$.

\subsubsection{Genotyping of the FMR1 locus in the CRMGEN-FraX prototype RMs}

Analysis of the CRMGEN-FraX prototype RMs was originally planned at the IRMM; however, the experience from CRMGEN ring trials showed that this assay is notoriously difficult no matter what protocol was employed. Therefore, following the stability studies samples were sent back to Manchester for analysis according to the reference [72]. Briefly, after PCR of the FMR1 locus (using 7-deaza dGTP instead dGTP with the primers FMR1-fw: 5'-TCC ggT ggA ggg CCg CCT CTg AgC-3', FMR1-rev: 5'-TTC AgC CCT gCT AgC gCC ggg AgC-3', giving a theoretical fragment of $159 \mathrm{bp}$ in an individual with zero repeats), the products were subjected to 6\% polyacrylamide gel electrophoresis. The gels were then visualised using a silver staining technique and evaluated by the Genescan software. The Fragile X samples were then analyzed by Southern Blot analysis using a radiolabelled probe to resolve alleles with high repeat numbers and also their methylation status.

\subsubsection{Analysis of the human coagulation Factor V G1691 “Leiden” mutation}

The Factor V G1691A “Leiden” mutation was genotyped using The LC Factor V Leiden Mutation Detection Kit with a LightCycler 1.5 instrument (Roche Diagnostics Belgium, Vilvoorde, Belgium) according to the manufacturer's instructions. In addition, the protocol was modified for a relative quantitation of the samples analyzed.

\subsubsection{Analysis of the Factor II gene G20210A variant}

Techniques applied for the analysis of the Factor II (prothrombin) gene G20210A mutation and for the characterisation of the candidate CRMs have been described previously in the certification reports on the CRMs IRMM/IFCC-490, -491, and -492 [II,III,IV] and other publications [I,V,VI].

\subsection{Field trials}

Field trials on the CRMGEN prototype RMs were carried out within the European Molecular Genetics Quality Network (EMQN). These samples were sent to the participating laboratories along with feedback forms to be filled out.

Protocols of the collaborative studies CS 246 and CS 262 on the candidate NIBSC-WHO Reference Panel samples were published by the NIBSC [65,67].

Design, organisation and evaluation of field trials on the candidate CRMs IRMM/IFCC-490, -491, and -492 have been reported elsewhere [I,II,III,IV,V] as well as the proficiency testing exercise using the plasmidic QCMs for the analysis of the G20210A variant in the prothrombin gene [VI]. 


\section{RESULTS}

\subsection{Results on the CRMGEN prototype RMs}

\subsubsection{CRMGEN-HH - PCR product based prototype RMs}

PCR product based prototype RMs produced for the hereditary haemochromatosis mutations C282Y and H63D in the HFE gene are specified in the Table 4.

Table 4. Summary table on the CRMGEN-HH prototype RMs processed and tested

\begin{tabular}{|c|r|c|c|c|}
\hline Prototype RM & \multicolumn{1}{c|}{ Vials } & C282Y / H63D genotype & Additive & Lyophilisation \\
\hline CRMGEN-HH RM01 & $1-180$ & wild-type / wild-type & - & Freeze-dried \\
\hline CRMGEN-HH RM01 & $181-360$ & wild-type / wild-type & - & - \\
\hline CRMGEN-HH RM02 & $1-180$ & wild-type / wild-type & SS DNA & Freeze-dried \\
\hline CRMGEN-HH RM02 & $181-360$ & wild-type / wild-type & SS DNA & - \\
\hline CRMGEN-HH RM03 & $1-180$ & heterozygous / heterozygous & - & Freeze-dried \\
\hline CRMGEN-HH RM03 & $181-360$ & heterozygous / heterozygous & - & - \\
\hline CRMGEN-HH RM04 & $1-180$ & heterozygous / heterozygous & SS DNA & Freeze-dried \\
\hline CRMGEN-HH RM04 & $181-360$ & heterozygous / heterozygous & SS DNA & - \\
\hline
\end{tabular}

Homogeneity: Although prototype RMs containing SS DNA gave clearer results (i.e. sharper bands, see Figure 7.), no significant differences were shown between the homogeneity of the different prototypes and the minimal sample intake was established as $0.5 \mu \mathrm{l}$.

Figure 7. Freeze-dried and liquid (NFD) CRMGEN-HH RMs without (RM01) and with SS DNA (RM02)

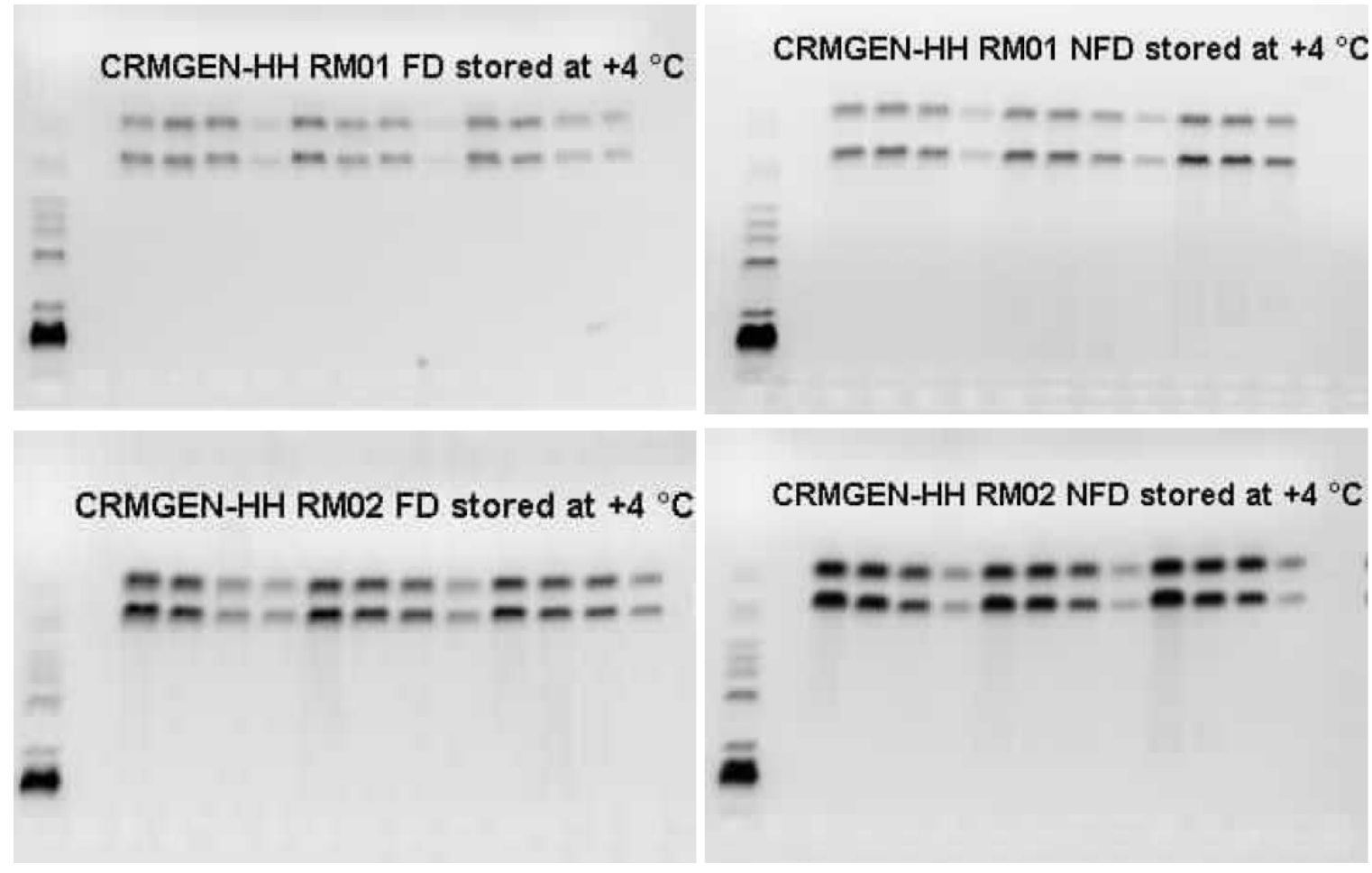


Field study: CRMGEN-HH prototype RMs were included in the EMQN EQA scheme (Aug 2003) for hereditary haemochromatosis including 45 labs across Europe. Thirty three labs returned results to Dublin. Although some participants of the trial reported clearer results from the RMs with carrier SS DNA, 9 of 33 labs (27\%) reported spurious results on the blank samples containing only SS DNA without human HFE sequences.

Stability: Target sequences with the expected genotypes were detected in each vial until the 1/1000 dilution even after 8 weeks of storage at $+18{ }^{\circ} \mathrm{C}$ or any other lower temperature. At these temperatures no differences were found in the stability of the different presentation formats (lyophilised or liquid, with or without salmon sperm DNA as additive). Lyophilised samples proved to be stable even at $+60{ }^{\circ} \mathrm{C}$ for 8 weeks, but all the liquid samples lost volume at this temperature and the detection of the target sequences were ambiguous after this period. In case of the stability study samples stored at $60{ }^{\circ} \mathrm{C}$, target sequences could not be detected at higher dilutions from the NFD prototype RMs containing SS DNA (data not shown).

One-year long-term stability study (LTSS) samples were analyzed using the multiplex real-time PCR method on the LightCycler. Comparing the different prototype RMs using two ways ANOVA with replication, no differences were found between the lyophilised and liquid RMs. On the other hand, the Cp values on the prototype RMs with salmon sperm DNA were significantly higher than the Cp values on prototypes without salmon sperm DNA (18.66 vs. $17.52 \mathrm{p}<0.05$ for the homozygous wild-type and 19.43 vs. 17.73 p<0.005 for the compound heterozygous RMs).

\subsubsection{CRMGEN-CF - Cell line based prototype RMs}

Master and working cell lines of EBV transformed lymphocytes with validated CF genotypes were stored in liquid $\mathrm{N}_{2}$ at IRMM, but one of the vials containing these cells was exploded in an incident. Although processing and stability testing of various cell line based prototype RMs were planned using different vials and lyoprotectants, the production of such cell pellet based materials were cancelled due to insurmountable regulatory barriers identified in handling, storage and transportation of such materials, which all require authorisation according to 'Biosafety Level 2’ [73].

\subsubsection{CRMGEN-FraX - Genomic DNA based prototype RMs}

Genomic DNA based prototype RMs produced for the fragile X syndrome representing a normal heterozygous female (Allele 1: (CGG) $)_{10}$, AGG, (CGG) ${ }_{11}$; Allele 2: (CGG) ${ }_{10}$, AGG, (CGG), AGG, (CGG) $)_{10}$ ) are listed in the Table 5.

Stability and homogeneity: All short- and long-term stability study samples tested by PCR/PAGE Genescan produced acceptable results regardless of extraction method, freeze drying or liquid medium and temperature/time stored. Southern blot testing showed that samples were stable at both $-20{ }^{\circ} \mathrm{C}$ and $4{ }^{\circ} \mathrm{C}$. However, phenol/chloroform extraction resulted in a slightly better stability and 
Puregene method in a superior purity of extracted DNA. The samples stored at $18{ }^{\circ} \mathrm{C}$ and $40{ }^{\circ} \mathrm{C}$ showed poor quality genotype test results in both series of Southern blot tests, thus stability is compromised at these temperatures. In addition, treatment at $65{ }^{\circ} \mathrm{C}$ for inactivation of nucleases resulted in DNA degradation in some phenol extracted samples.

Field trial: All prototype RMs performed well; however some centres preferred the phenol extracted DNA.

Table 5. Summary table on the CRMGEN-FraX prototype RMs processed and tested

\begin{tabular}{|c|r|l|c|}
\hline Prototype RM & \multicolumn{1}{c|}{ Vials } & \multicolumn{1}{c|}{ DNA extraction method } & Lyophilisation \\
\hline CRMGEN-FraX RM01 & $1-200$ & NIBSC phenol/chloroform extraction & Freeze-dried \\
\hline CRMGEN-FraX RM01 & $201-400$ & NIBSC phenol/chloroform extraction & - \\
\hline CRMGEN-FraX RM02 & $1-200$ & sodium perchlorate/chloroform extr. & Freeze-dried \\
\hline CRMGEN-FraX RM02 & $201-400$ & sodium perchlorate/chloroform extr. & - \\
\hline CRMGEN-FraX RM03 & $1-200$ & Mcr phenol/chloroform extraction & Freeze-dried \\
\hline CRMGEN-FraX RM03 & $201-400$ & Mcr phenol/chloroform extraction & - \\
\hline CRMGEN-FraX RM04 & $1-200$ & Mcr Puregene extraction & Freeze-dried \\
\hline CRMGEN-FraX RM04 & $201-400$ & Mcr Puregene extraction & - \\
\hline
\end{tabular}

\subsubsection{CRMGEN-FV - Plasmidic prototype RMs}

Plasmidic prototype RMs produced for the analysis of the human coagulation Factor V G1691A “Leiden” mutation are summarised in the Table 6.

Table 6. Summary table on the CRMGEN-FV plasmidic prototype RMs processed and tested

\begin{tabular}{|l|c|l|c|}
\hline \multicolumn{1}{|c|}{ Prototype RM } & \multicolumn{1}{c|}{ TE-Buffer } & \multicolumn{1}{c|}{ Additive } & Lyophilisation \\
\hline CRMGEN-FV RM01 & $1 \mathrm{mM}: 0.1 \mathrm{mM}, \mathrm{pH} 7.5$ & - & Freeze-dried \\
\hline CRMGEN-FV RM02 & $1 \mathrm{mM}: 0.1 \mathrm{mM}, \mathrm{pH} 7.5$ & - & - \\
\hline CRMGEN-FV RM03 & $1 \mathrm{mM}: 0.1 \mathrm{mM}, \mathrm{pH} 7.5$ & $10 \%$ of sucrose & Freeze-dried \\
\hline CRMGEN-FV RM04 & $1 \mathrm{mM}: 0.1 \mathrm{mM}, \mathrm{pH} 7.5$ & $50 \%$ of glycerol & - \\
\hline CRMGEN-FV RM05 & $1 \mathrm{mM}: 0.1 \mathrm{mM}, \mathrm{pH} 7.5$ & crosslinked SS DNA & Freeze-dried \\
\hline CRMGEN-FV RM01a & $10 \mathrm{mM}: 1 \mathrm{mM}, \mathrm{pH} 8.0$ & - & Freeze-dried \\
\hline CRMGEN-FV RM02a & $10 \mathrm{mM}: 1 \mathrm{mM}, \mathrm{pH} 8.0$ & - & - \\
\hline CRMGEN-FV RM03a & $10 \mathrm{mM}: 1 \mathrm{mM}, \mathrm{pH} 8.0$ & $10 \%$ of sucrose & - \\
\hline
\end{tabular}

In the case of CRMGEN-FV RM05 carrier SS DNA was treated using Psoralen/UVA-crosslinking to prevent amplification of salmon DNA sequences. Furthermore, concentration of the CRMGEN-FV prototype RMs was set to a relatively low copy number, in order to increase the sensitivity of the stability tests. 
Homogeneity and stability: Analysis of the $1^{\text {st }}$ batch of CRMGEN-FV prototype RMs prior to the lyophilisation revealed a significant degradation of target sequences during the transport and 4 months storage at $+4^{\circ} \mathrm{C}$. RM02 and RM03 with $10 \%$ of sucrose gave relative low signals, but still could be genotyped. On the other hand, the prototype RM04 and RM05 could not be reliably genotyped (often only one of the alleles was detected) due to the degradation of target sequences. In addition, melting peaks on the prototypes RM03 and RM04 (containing sucrose or glycerol, respectively) were shifted towards lower temperature. Therefore the new batches of CRMGEN-FV prototype RMs were prepared only in 3 formats. This $2^{\text {nd }}$ batch however was dissolved in a 10-times concentrated TE buffer (10 mM Tris : 1 mM EDTA) at pH 8.0, which performed better in the feasibility study.

Table 7. Stability and homogeneity of the CRMGEN-FV prototype RMs: Cp values on the undiluted samples after storage at various times and temperatures.

The homogeneity was determined from the results obtained on the samples stored at $-20{ }^{\circ} \mathrm{C}$ for various times and those stored at the reference temperature $\left(-70^{\circ} \mathrm{C}\right)$. [ND: not determined; NS: not significant]

\begin{tabular}{|c|c|c|c|c|c|}
\hline RM01a & \multicolumn{5}{|c|}{ Temperature } \\
\hline Time & $-70^{\circ} \mathrm{C}$ & $-20^{\circ} \mathrm{C}$ & $+4^{\circ} \mathrm{C}$ & $+18^{\circ} \mathrm{C}$ & $+40^{\circ} \mathrm{C}$ \\
\hline Homogeneity & \multicolumn{2}{|c|}{$34.33 \pm 1.80$} & & & \\
\hline 2 weeks & & $31.59 \pm 1.14$ & $35.09 \pm 0.37$ & $36.94 \pm 3.17$ & ND \\
\hline 4 weeks & & $34.82 \pm 0.71$ & $33.42 \pm 0.73$ & $36.33 \pm 1.13$ & ND \\
\hline 8 weeks & $34.90 \pm 0.96$ & $35.11 \pm 0.85$ & $35.53 \pm 1.25$ & $37.96 \pm 0.59$ & ND \\
\hline Evaluation & & NS & NS & $\mathbf{p}<\mathbf{0 . 0 1}$ & $! !$ \\
\hline RM02a & \multicolumn{5}{|c|}{ Temperature } \\
\hline Time & $-70^{\circ} \mathrm{C}$ & $-20^{\circ} \mathrm{C}$ & $+4^{\circ} \mathrm{C}$ & $+18{ }^{\circ} \mathrm{C}$ & $+40^{\circ} \mathrm{C}$ \\
\hline Homogeneity & \multicolumn{2}{|c|}{$31.24 \pm 1.45$} & & & \\
\hline 2 weeks & & $31.73 \pm 0.49$ & $29.69 \pm 0.85$ & $32.24 \pm 0.67$ & ND \\
\hline 4 weeks & & $30.72 \pm 1.54$ & $31.68 \pm 0.14$ & $32.80 \pm 0.88$ & ND \\
\hline 8 weeks & $30.34 \pm 0.32$ & $32.19 \pm 0.48$ & $32.08 \pm 1.11$ & $32.68 \pm 3.00$ & ND \\
\hline Evaluation & & NS & NS & NS & $! !$ \\
\hline RM03a & \multicolumn{5}{|c|}{ Temperature } \\
\hline Time & $-70^{\circ} \mathrm{C}$ & $-20^{\circ} \mathrm{C}$ & $+4^{\circ} \mathrm{C}$ & $+18^{\circ} \mathrm{C}$ & $+40^{\circ} \mathrm{C}$ \\
\hline Homogeneity & \multicolumn{2}{|c|}{$26.15 \pm 0.49$} & & & \\
\hline 2 weeks & & $26.81 \pm 0.35$ & $25.34 \pm 0.34$ & $26.16 \pm 0.12$ & $27.59 \pm 0.26$ \\
\hline 4 weeks & & $25.98 \pm 0.13$ & $26.28 \pm 0.25$ & $27.18 \pm 0.39$ & $27.70 \pm 0.39$ \\
\hline 8 weeks & $25.83 \pm 0.09$ & $25.99 \pm 0.33$ & $25.87 \pm 0.29$ & $27.35 \pm 0.29$ & $29.34 \pm 0.43$ \\
\hline Evaluation & & NS & NS & $p<0.0001$ & $p<0.00001$ \\
\hline
\end{tabular}

Homogeneity testing of the $2^{\text {nd }}$ batch of CRMGEN-FV prototype RMs resulted in significantly higher Cp values on the prototypes RM01a and RM02a (Table 7.), indicating a serious loss of the quantifiable target sequences in these prototypes during the processing. Comparison of these data to the results on the raw plasmid preparations $(\mathrm{Cp}=24.63 \pm 0.30)$ revealed that aliquotation of the material into small vials lead already to a dramatic increase of the Cp values. In case of RM03a, this 
difference between the samples stored at the reference temperature and the raw plasmid preparation received from Leiden was much lower.

Target sequences could not be amplified from the RM01a and RM02a samples stored at $+40{ }^{\circ} \mathrm{C}$. Moreover, in these prototypes often only one of the two alleles could be detected. Prototype RM03a showed always the expected heterozygous genotype and also its $\mathrm{Cp}$ value exhibited a smaller standard deviation. Following this initial increase in the Cp values, all of the $2^{\text {nd }}$ batch CRMGEN-FV prototype RMs were stable at $-20{ }^{\circ} \mathrm{C}$ and the RM03a also at $+4{ }^{\circ} \mathrm{C}$.

In the case of the $2^{\text {nd }}$ lots of CRMGEN-FV RMs, melting temperature (Tm) values on a given allele displayed only negligible variation (data not shown), which might be attributed to minor variations in the reaction mix components but do not affect the correct assignment of the genotype.

\subsection{Analysis of the NIBSC-WHO candidate Reference Panel samples}

Genotyping of the candidate Reference Panel samples for the respective sequence variant gave the expected genotypes from each vials; however, a significant shift in the melting temperature (Tm) towards lower temperatures was detected in both set of NIBSC-WHO samples using the LC Factor V Leiden Mutation Detection Kit and different lots of the LC Prothrombin G20210A Mutation Detection Kit with the LightCycler technique (Figure 8.) and an unusual Taqman allelic discrimination plot could be observed using the Taqman allelic discrimination assay on the ABI Prism 7700 (data not shown).

Figure 8. Melting peaks on the NIBSC-WHO Reference Panel Samples

A: Melting peaks on the NIBSC-WHO Reference Panel for the Factor V Leiden mutation: homozygous wild-type sample (-•-) compared to the LC kit control (---) and to a CRMGEN-FraX prototype RM sample (-) both carrying the homozygous wild-type genotype $\left[\Delta \mathrm{Tm}=\right.$ ca. $\left.-1.7^{\circ} \mathrm{C}\right]$

B: Melting peaks on the NIBSC-WHO Reference Panel for the Factor II G20210A mutation: heterozygous

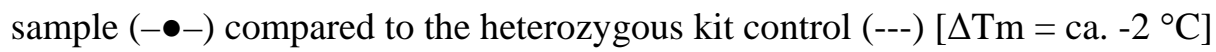
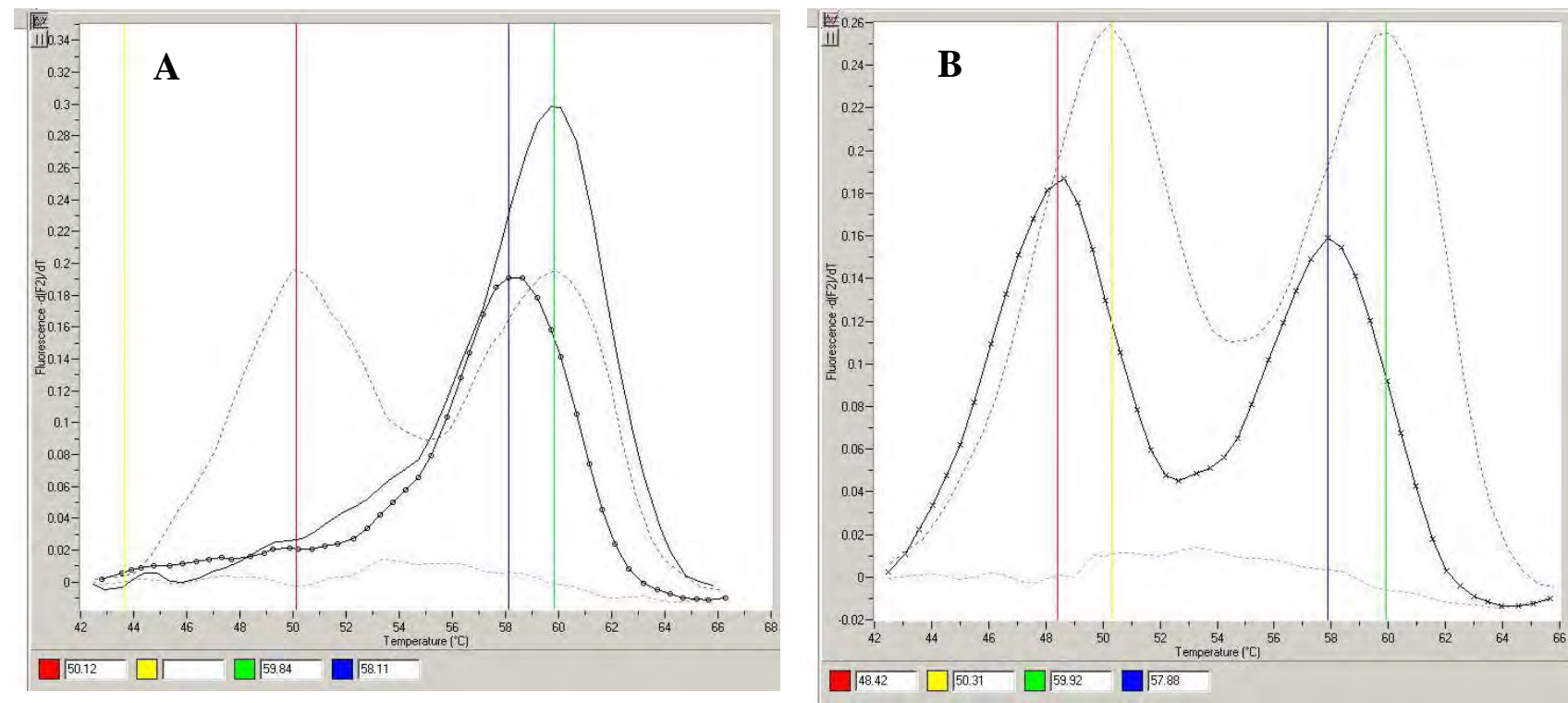
This shift of $\mathrm{Tm}$ is lower than the $\pm 2.5^{\circ} \mathrm{C}$ value specified in the pack insert as possible difference between samples and the control provided in the kit, but very much exceeds the total precision of the kit (median CV of $0.36 \%$ assessed according to NCCLS EP5-A guideline). The observed phenomenon was further investigated, in order to avoid similar issues. Subsequent experiments showed that (i) testing of these gDNA-based RMs for other polymorphisms, the extent of the Tm shift was similar; (ii) the Tm-shift is linked to the quantity of RM engaged in the PCR reaction (could be abolished by 1:10 dilution of the samples) and (iii) cannot be attributed to the trehalose, which was used as lyoprotectant in these RMs [64,66] (see section 4.3.1.!).

\subsection{Feasibility study on the preservation of plasmidic DNA}

\subsubsection{Effect of the additives}

Different concentrations of TE-Buffer (10 mM : $1 \mathrm{mM}$; pH 8.0 and $1 \mathrm{mM}: 0.1 \mathrm{mM}$; pH 8.0) and most of the tested compounds did not have any influence either on the amplification of target DNA sequence or on the Tm values.

Neither trehalose nor most of the other carbohydrates tested (fructose, mannitol, sucrose, sorbitol) had an effect on the melting peaks, when they were added to the reaction mix directly or once the materials had been freeze dried properly. However, glycerol led to a very significant decrease in the melting temperatures and consequently to seriously impaired amplification efficiency at the concentration, which should be applied for an effective cryoprotection. In addition, NFD or inadequately lyophilised (moisture content $>>1 \%$ ) prototype RMs containing sucrose showed a significant Tm-shift on the wild-type FII sequence (59.33 $\pm 0.20^{\circ} \mathrm{C}$ vs. $\left.60.20 \pm 0.06{ }^{\circ} \mathrm{C}\right)$, when they were tested after 12 weeks storage at $-20^{\circ} \mathrm{C}$ or $+4{ }^{\circ} \mathrm{C}$ ).

The ionic detergent sodium dodecyl sulphate (SDS) used for DNA preservation in some formulations at $1 \%$ completely inhibited the amplification at $0.01 \%$.

Interestingly, addition of $1 \% \mathrm{Na}$-ascorbate to the prototype $\mathrm{RM}$ resulted in a significant decrease of the $C p$ values $(C p=13.46 \pm 0.21$ vs. $C p=14.37 \pm 0.25$; $p<0.05)$ without affecting the Tm values.

\subsubsection{Freeze-thaw tolerance of plasmidic prototype RMs}

There was no significant difference between prototype RM samples passed 10 freeze-thaw cycles or stored at $4{ }^{\circ} \mathrm{C}$.

\subsection{Production and certification of the candidate CRMs IRMM/IFCC-490, -491 and -492}

\subsubsection{Construction and characterisation of the reference plasmids}

Construction, sequencing and characterisation of the reference plasmids pIRMM-0001 and pIRMM-0002 have been described in details previously [I,II,III,IV,V]. Map of the pUC18 reference plasmids pIRMM-0001 and pIRMM-0002 is shown on the Figure 9. 
Figure 9. Map of the reference plasmid pIRMM-0001 (3311 bp, wild-type). The Factor II mutation G20210A is located at position 943 in the mutant reference plasmid pIRMM-0002.

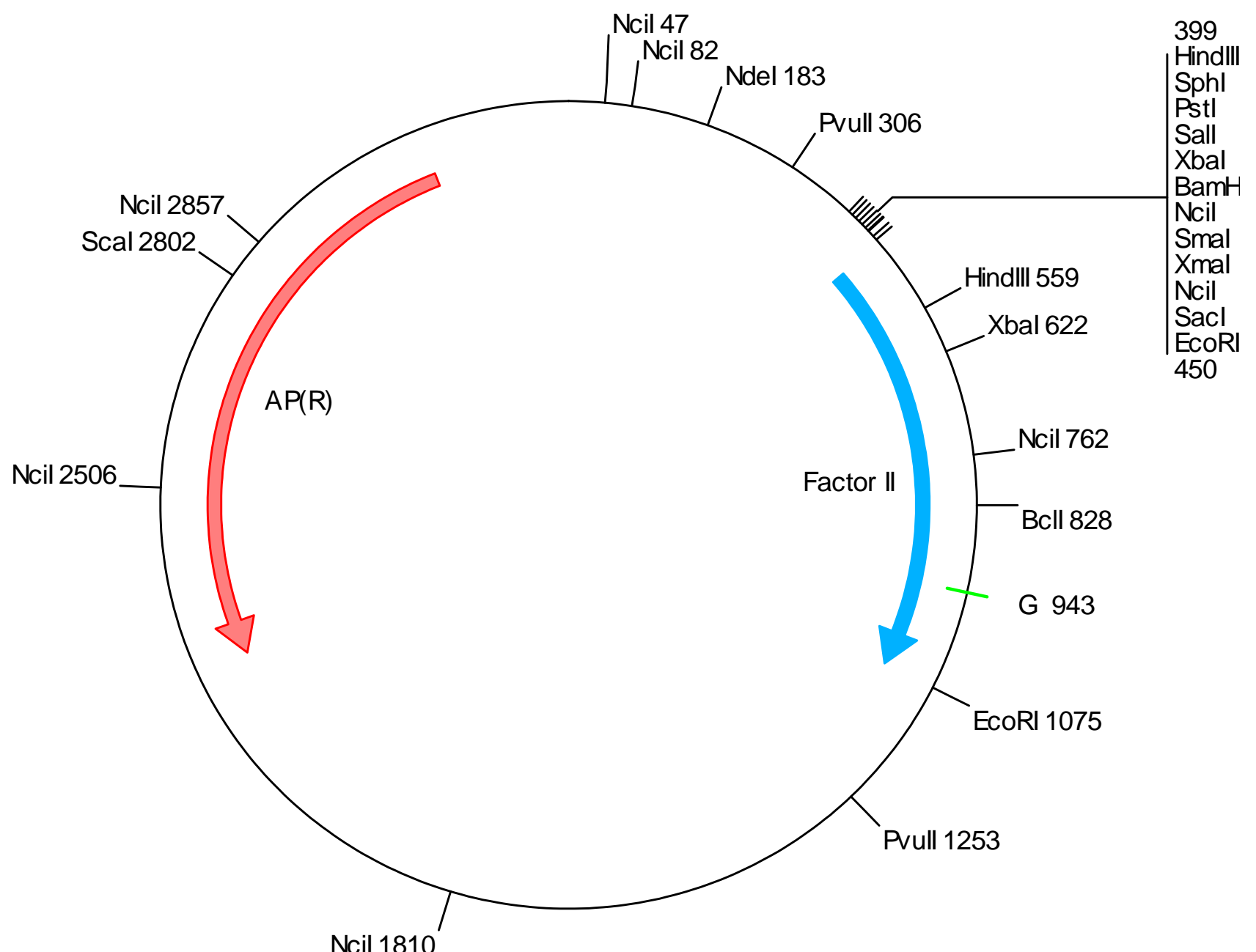

Sequencing: Sequence analysis (Figure 10.) of the selected fragments showed that the isolated 609 bp length sequences were identical to the GenBank reference sequence (accession number M17262, nt 26302 to 26910) except for the G20210A mutation (corresponding to the nt 26784 in the reference sequence) and an $A \rightarrow G$ point mutation at position 20054 (nt 26628 in the GenBank reference sequence). Interestingly, this second mutation was already present in the patient sample and was linked to the mutated sequence. The cloned wild-type fragment corresponded to $100 \%$ to the reported prothrombin gene sequence.

\subsubsection{Evaluation of the $1^{\text {st }}$ batch of the candidate FII CRMs}

Short-term stability: Analysis of the short-term stability study samples produced no or weak signals using the LightCycler platform and kit for the G20210A mutation detection (data not shown). This applied as well for samples stored at $-70{ }^{\circ} \mathrm{C}$ and $-20^{\circ} \mathrm{C}$. These results indicated that stability of the candidate CRMs was an issue and the presentation format had to be re-investigated towards the processing of a new lot of the CRMs IRMM/IFCC-490, -491 and -492. 
Figure 10. Identity analysis of the homology between the sequence of the 609 bp gene insert carrying the 20210A mutation (Query) with the GenBank reference sequence (Sbjct) of the prothrombin gene (accession number M17262, nt 26302 to 26910) on the website www.ncbi.nlm.nih.gov. Mutations are highlighted in bold.

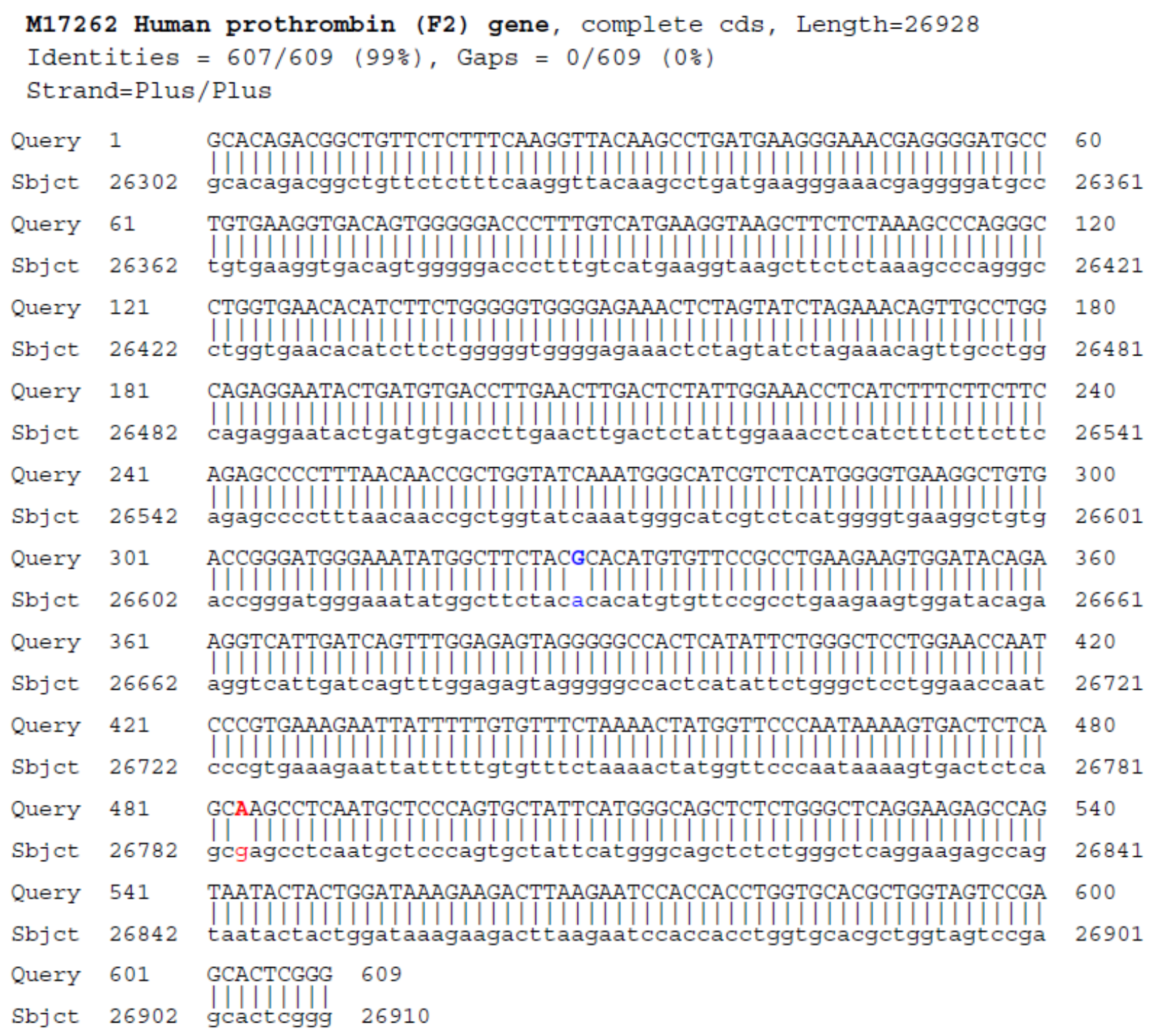

Commutability / fitness for purpose: Outcome of the field trial on the reference plasmids were published previously [I]. For the analysis of the samples 9 different methods were used and every RM was genotyped correctly. The study showed that plasmid-type RMs containing defined point mutations can be produced and successfully used in tests for detection of point mutations in a typical QA scheme. However, feedbacks from the participants suggested a higher concentration of the target sequence as well as to set the minimal sample intake to $\leq 1 \mu \mathrm{l}$.

\subsubsection{Characterisation of the new lot of the CRMs IRMM/IFCC-490, -491 and -492}

Processing, characterisation and certification of the new batch of the FII CRMs have been published previously [V] and described in details in the certification reports [II,III,IV]. Following a novel approach, the new batch of these RMs were produced as liquid frozen materials containing 
approximately $1 \mathrm{ng}$ plasmid DNA in a volume of $50 \mu \mathrm{L}$ of a Tris/EDTA solution (10 mM Tris : 1 mM EDTA, pH 8.0) in polypropylene vial. This batch was characterised according to the requirements of the ISO Guides 34 and 35 [31,34].

Homogeneity study: Homogeneity of these candidate CRMs was evaluated from results on the short-term stability samples stored at $-20^{\circ} \mathrm{C}$ and/or at the reference temperature $\left(-70^{\circ} \mathrm{C}\right)$ summarised in Table 8.

Table 8. Cp values and melting peaks on the $2^{\text {nd }}$ batch of FII CRMs using the LightCycler platform

\begin{tabular}{|l|c|c|c|}
\hline & IFCC/IRMM-490 & IFCC/IRMM-491 & IFCC/IRMM-492 \\
\hline $\mathrm{Cp}( \pm \mathrm{SD})$ value & $14.64( \pm 0.26)$ & $15.15( \pm 0.39)$ & $15.16( \pm 0.42)$ \\
\hline $\mathrm{Tm}( \pm \mathrm{SD})_{\mathrm{G}-\text {-allele }}$ & $59.49( \pm 0.11){ }^{\circ} \mathrm{C}$ & N.A. & $59.76( \pm 0.20){ }^{\circ} \mathrm{C}$ \\
\hline $\mathrm{Tm}( \pm \mathrm{SD})_{\mathrm{A} \text {-allele }}$ & N.A. & $49.98( \pm 0.14){ }^{\circ} \mathrm{C}$ & $50.29( \pm 0.16)^{\circ} \mathrm{C}$ \\
\hline$\Delta \mathrm{Tm}( \pm \mathrm{SD})_{\mathrm{G} \& A-\text { alleles }}$ & N.A. & N.A. & $9.47( \pm 0.11){ }^{\circ} \mathrm{C}$ \\
\hline
\end{tabular}

A plot of the Cp values of the PCR products versus sample number did not reveal a trend in the filling sequence of the vials (data not shown). The slope of the regression line was not statistically different from zero ( $t$-test at 95\% confidence interval) and the data followed a normal and unimodal distribution (data not shown) which indicated that the candidate CRMs were homogeneous. The homogeneity-related uncertainty on the DNA quantity per vial throughout the batches was inferior to $10 \%$ for all three materials. Every sample and its dilutions at least down to 1:10000 showed the expected melting curves corresponding to the genotype of the candidate CRM samples. This proved that the candidate CRMs are sufficiently homogeneous and that the minimal sample intake can be as low as $0.5 \mu \mathrm{L}$.

Stability: Condition of every sample was good, loss of volume or other changes could not be observed. Every melting curve corresponded to the expected genotype and no Tm-shift was detected. These results and the $\mathrm{Cp}$ values indicated a sufficient short-term (8 weeks at $-20^{\circ} \mathrm{C}, 4^{\circ} \mathrm{C}$ and $18{ }^{\circ} \mathrm{C}$ ) and long-term (6, 12 and 24 months at $-20^{\circ} \mathrm{C}$ and $4{ }^{\circ} \mathrm{C}$ ) stability of these candidate CRMs at the tested temperatures [II,III,IV,V]. However, instead of an increase of the Cp values due to a degradation of the target DNA sequences, lower Cp values were recorded on samples stored at higher temperatures and this trend was found to be statistically significant (Figure 11.). In addition, less dilution steps gave appropriate results for the samples stored at $-20{ }^{\circ} \mathrm{C}$ or at the reference temperature, than for samples stored at higher temperatures. These observations could be explained by better accessibility or lower adsorption rate of target sequences in samples stored at higher temperatures.

Field study: In order to test the performance of the 3 candidate CRMs, they were sent to 7 diagnostic laboratories that used routinely different genotyping methods for the detection of the 
prothrombin G20210A variant. All DNA-containing samples were genotyped correctly and unambiguously demonstrating that the materials are fit for their intended purpose.

Figure 11. Cp values on the short-term stability samples stored at different temperatures
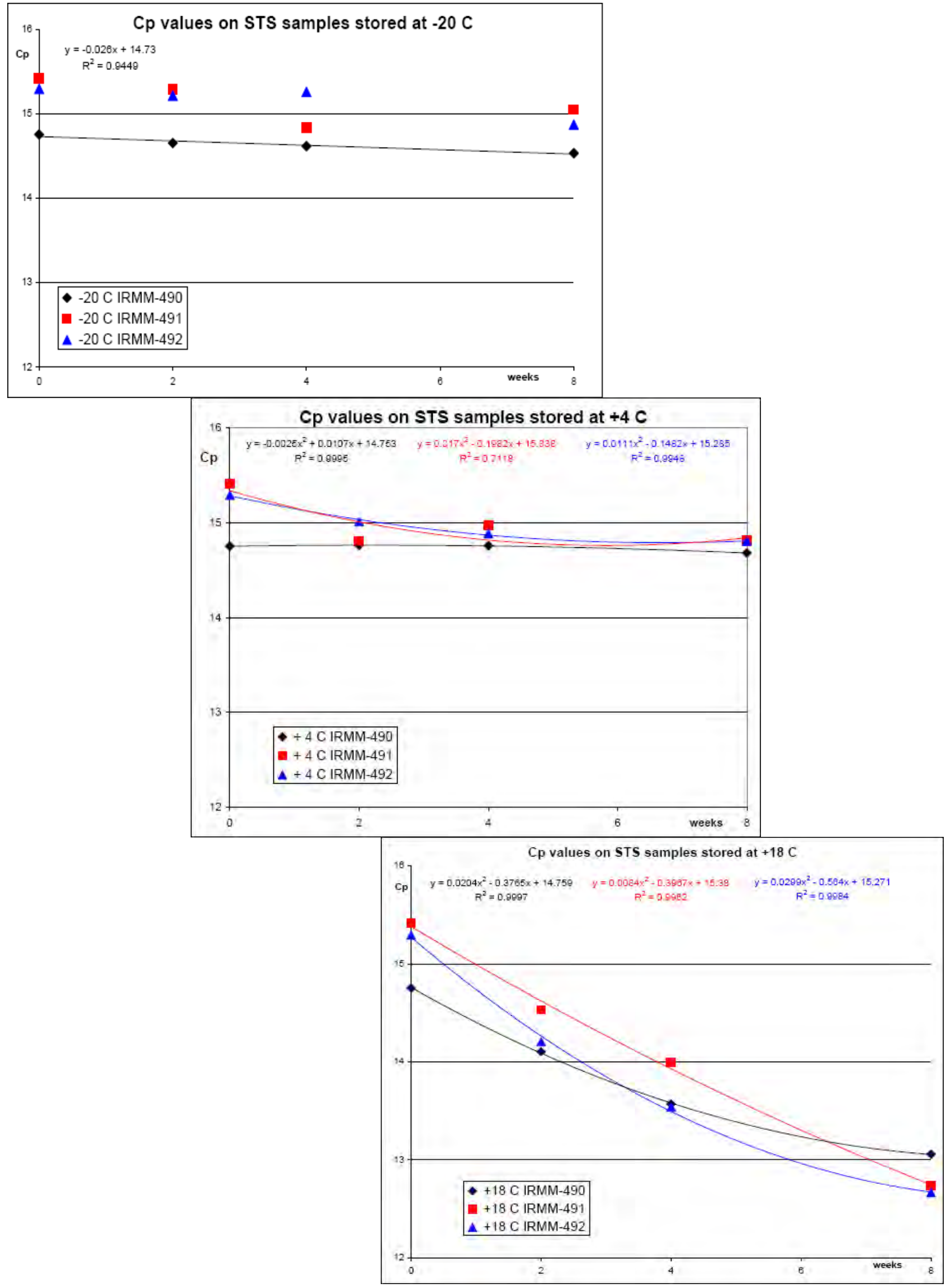
Certification: The identity of the gene fragments inserted in the plasmids was assessed by internal and external bi-directional sequencing with a probability of misreadings estimated to be $\mathrm{p}<(1 / 609)^{2}$. This estimation is based on the agreement between forward and reverse sequencing of the 609-bp fragments inserted in the pUC18 vectors. DNA concentration has been indicated in the certificate as additional information regarding the CRMs, but it is not displayed as a certified property value. The sample intake recommended is 0.5 to $5 \mu \mathrm{L}$. The stability of the CRMs is being followed in the postcertification monitoring and the certification is valid for one year after purchase of the unit.

\subsection{Proficiency testing study using QCMs containing rare sequence variants}

The introduction of new mutations into the pIRMM-0001 (wild-type) FII reference plasmid, the production of the QCMs as well as the organisation and evaluation of the proficiency testing study has been published in details earlier [VI].

\subsubsection{Preparation of the samples for the proficiency testing exercise}

Following the site-directed mutagenesis procedure, screening of plasmid preparations detected two new variants displaying unusual melting peaks at ca. $54{ }^{\circ} \mathrm{C}$ and at $58{ }^{\circ} \mathrm{C}$ [VI]. Sequencing of these plasmids identified the newly introduced mutations identified as the C20209T and the [20175T>G; 20179_20180delAC] mutations, respectively. Using these plasmids and the FII reference plasmids four QCMs were produced containing $10 \mathrm{pg} / \mu \mathrm{L}$ plasmid DNA in $30 \mu \mathrm{L}$ TE-buffer (10 mM : $1 \mathrm{mM}$; pH 8.0; autoclaved):

Sample A: 20210A / [20175G; 20179_20180delAC] - heterozygous for the G20210A mutation

Sample B: G20210 / 20209T - homozygous wild-type for the G20210A mutation

Sample C: 20210A / 20210A - homozygous for the G20210A mutation

Sample D: G20210 / G20210 - homozygous wild-type for the G20210A mutation

Melting curves obtained on samples A and B using the LightCycler are shown in Figure 12.

Figure 12. Unusual melting curves on the proficiency testing samples 'Sample A' and 'Sample B'

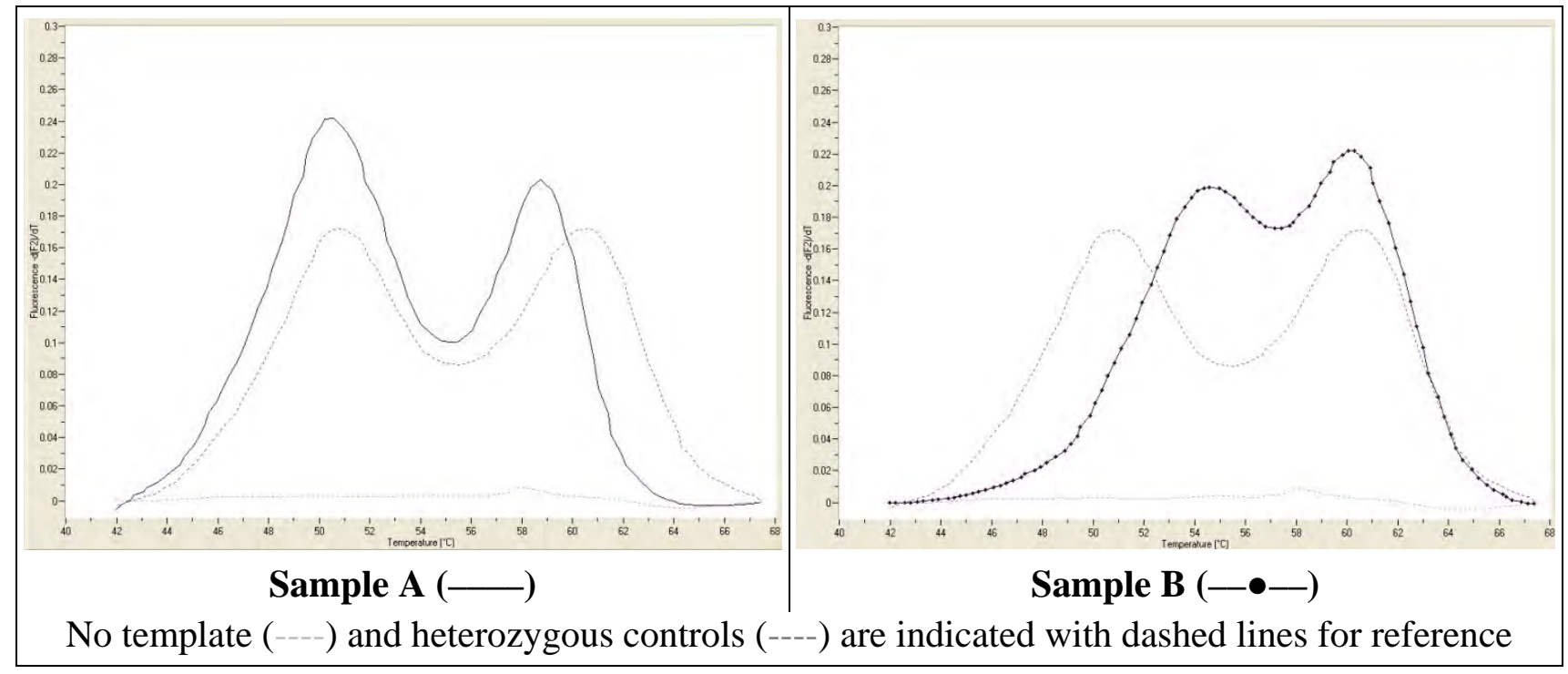




\subsubsection{The proficiency testing exercise}

After removing of duplications resulting from the overlaps of the EQA schemes, 189 laboratories from 21 countries participated to the study. These participants in the survey applied a large variety of genotyping techniques representing 50 different analytical procedures with sample intakes ranging $0.5-10 \mu \mathrm{L}$. All of the assays could amplify the target sequence from the plasmids proving that the samples were suitable for the interlaboratory comparison exercise.

Genotyping results can be split into usual and unusual results after analysis (Table 9.) Usual results are results of methods, which were not influenced by the mutations in samples A and B. In 67 and 85 laboratories for samples A and B respectively the mutations affected the methods and unusual genotyping results were obtained which should have been recognised and reported accordingly.

Table 9. Overview on the genotyping results and error rates

\begin{tabular}{|c|c|c|c|c|c|}
\hline & Sample $A^{a}$ & Sample B & Sample C & Sample D & Total \\
\hline Usual results & 112 & 104 & 189 & 189 & 596 \\
\hline Correct & 102 & 100 & 177 & 180 & 559 \\
\hline False & 7 & 3 & 12 & 8 & 30 \\
\hline Not reported & 3 & 1 & - & 1 & 5 \\
\hline Error rate & $6.4 \%$ & $2.9 \%$ & $6.3 \%$ & $4.3 \%$ & $5.1 \%$ \\
\hline Unusual results & 67 & 85 & n. a. & n. a. & 152 \\
\hline Not reported (false) & $50(74.6 \%)$ & $33(38.8 \%)$ & n. a. & n. a. & 83 (54.6 \%) \\
\hline Technical issue $^{b}$ & $10(14.9 \%)$ & $22(25.9 \%)$ & n. a. & n. a. & 32 (21.1\%) \\
\hline Recognised variant & 7 (10.5 \%) & $30(35.3 \%)$ & n. a. & n. a. & 37 (24.3\%) \\
\hline
\end{tabular}

${ }^{a}$ Without Allelic Discrimination assays affected by an impaired amplification of the "wild-type" sequence due to the [20175T>G; 20179_20180delAC] mutation

${ }^{b}$ Refers to laboratories not reporting genotypes but describing observations such as unusual results of presumed technical origin

Among the usual results, the overall error rate was 5.1\%. Interestingly, the error rates were higher on the samples containing the G20210A mutant allele (6.4\% and $6.3 \%$, respectively) than on samples B and D without the G20210A mutation (2.9\% and 4.3\%, respectively); however, this difference was not statistically significant.

Using also the specifications of the methods applied and the raw data (e.g. copy of the gels, melting curves) on the genotyping assays, we could carry out a detailed analysis of the results to identify the sources of errors. This analysis revealed that the majority of the false results arose from the inadvertence of laboratory personnel and not from technical failure of the method as such. However, the allele-specific PCR assays proved to be less robust than other techniques and the elevated error rate of certain LDTs indicates that they, as a group, have to be more carefully validated. 
In the case of the unusual results, although a majority (54.6\%) of laboratories did not report when they experienced unusual results on samples A and B, other participants recognised the presence of the sequence variants (24.3\%) or attributed the unusual behaviour of the assay to technical issues (21.1\%), including poor quality or low concentration of the DNA [VI]. However, the atypical melting peak on the [20175G; 20179_20180delAC] mutation was reported by only 25.4\% of the participants probably because of the small deviation from the expected values (ca. $1.7{ }^{\circ} \mathrm{C}$ lower Tm than expected for the wild-type allele). Approximately half of these laboratories noted that such unusual melting peaks could indicate a variant in the probe region.

Table 10. displays the use of different genotype controls in the participant laboratories.

Table 10. Positive control samples used in the participating laboratories

\begin{tabular}{|c|c|c|c|c|c|}
\hline \multicolumn{6}{|l|}{ Positive controls used } \\
\hline & $\mathrm{n}$ & G20210 & G20210/20210A & $20210 \mathrm{~A}$ & rare SNPs \\
\hline Kit control & 106 & - & + & - & - \\
\hline G20210/20210A patient DNA & 35 & - & + & - & - \\
\hline $\begin{array}{l}\text { G20210 \& G20210/20210A patient } \\
\text { DNA }\end{array}$ & 18 & + & + & - & - \\
\hline $\begin{array}{l}\text { G20210/G20210 \& 20210A/20210A } \\
\text { patient DNA }\end{array}$ & 1 & + & - & + & - \\
\hline $\begin{array}{l}\text { G20210, G20210/20210A \& 20210A } \\
\text { patient DNA }\end{array}$ & 14 & + & + & + & - \\
\hline $\begin{array}{l}\text { G20210/20210A patient DNA \& } \\
5 \text { plasmids for rare SNPs }\end{array}$ & 1 & - & + & - & + \\
\hline Ring trial samples & 3 & + & + & + & - \\
\hline NIBSC-WHO reference panel* & 1 & + & + & + & - \\
\hline No data & 10 & & & & \\
\hline & & 37 & 178 & 19 & 1 \\
\hline
\end{tabular}

* At the time of our ring trial, the NIBSC-WHO Reference Panel for the Factor II G20210A mutation had not been commercialised. The RMs used by the laboratory could be the rest of the samples from the collaborative study. 


\section{DISCUSSION}

Advances in molecular pathology and biotechnology made DNA-based assays an important area of laboratory medicine and indispensable in patient care. Although DNA tests have a high impact on clinical decision-making and the number of tests carried out in diagnostic laboratories increases rapidly, validation and proper QA of molecular genetic tests are critically dependent on the availability of appropriate (C)RMs [7,74]. Therefore, the primary objectives of the projects presented were the development and production of DNA-based (C)RMs for clinical genetic testing and to elaborate the methodology to produce (C)RMs for any molecular genetic test.

In addition to an appropriate type and the overall suitability of the RMs, homogeneity and stability are by definition fundamental requirements for any RMs and have to be guaranteed by the producer [34]. Although the homogeneity of RMs for qualitative molecular genetic tests was not a critical problem, to ensure a reasonable shelf life became a crucial issue. Beside enzymatic breakdown of the DNA - which was prevented by proteinase digestion and/or heat denaturation of nucleases several mechanisms of physical/chemical degradation have been described, such as shearing during freezing, hydrolytic/oxidative cleavage, its enhanced form in the presence of metal ions, and depurination $[75,76]$. The rate of these processes depends mainly on the storage conditions of which optimisation for long-term DNA preservation required comprehensive studies.

Observations on the PCR-product based CRMGEN-HH prototype RMs confirmed that freeze drying can assure the stability of DNA-based RMs even at higher temperatures. On the other hand, the use of carrier SS DNA gave conflicting results (i.e. sharper bands on the gels, but no signals on higher dilutions) in the short-term stability study indicating the need for a more reliable assay for the comparison of the stability study samples, which enables a relative quantification of the sequences of interest in the different CRMGEN-HH prototype RMs. Analysis of one-year long-term stability samples using the improved multiplex real-time PCR method revealed that the concentration of target sequences was significantly lower in the liquid prototype RMs containing SS DNA displaying an impaired stability of such RMs. These data clearly disproved the speculation that salmon sperm DNA can improve the stability of small DNA molecules. Moreover, the field study on the CRMGEN-HH prototype RMs showed that SS DNA contains some amplifiable sequences under certain conditions, which might interfere with some techniques. In the case of further prototype RMs, this issue was avoided either by crosslinking the SS DNA or replacing it for bacteriophage lambda DNA. Unfortunately, despite the promising results on the CRMGEN-HH prototype RMs, the development of candidate CRMs for the analysis of the C282Y and H63D variants had been arrested due to IPR issues [7,55].

The cell-line based CRMGEN-CF RMs proved unfeasible due to the strict biohazard compliance and regulatory criteria applied to EBV transformed cells. Such cell lines however could provide excellent 
starting material for the preparation of gDNA based (C)RMs [77] as seen in the case of CRMGEN-FraX prototype RMs. Even if the 'Level 2' biohazard classification of EBV transformed materials obviated the preparation of cell pellet RMs for given rare mutations responsible for hereditary diseases, genotyped and well characterised cell lines established without using EBV or similar infectious agents (e.g. malignant cell lines) could be used as RMs for common sequence variants [51,52].

Genomic DNA has proven to be a suitable type of RMs for various genotyping techniques, such as PCR and Southern blot. Among the DNA extraction techniques tested, the Puregene extraction method yielded a superior purity with comparable stability of extracted DNA. Thus, this technique was chosen later on for the production of the $1^{\text {st }}$ WHO international genetic reference panel for Fragile X syndrome already brought through the WHO certification route [78]. Such a RM made of gDNA is of pivotal importance for the fragile $X$ syndrome as Southern blot is the reference/confirmation method used for the diagnosis [59]. Although the National Institute of Standards and Technology (NIST) have released a Standard Reference Material (SRM) for Fragile X syndrome to the market, the SRM 2399 is a set of 9 PCR products containing different number of CGG repeats [79], which is rather seen as a RM for screening purposes.

The plasmidic CRMGEN-FV prototype RMs were developed utilizing the experiences accumulated on the CRMGEN prototype RMs and some early results of the feasibility study on the preservation plasmidic DNA. Nevertheless, a relatively low copy number of plasmids were applied to increase the sensitivity of the stability studies. The significantly higher Cp values on the lyophilised CRMGEN-FV RM01a samples than the results obtained on the corresponding liquid RM02a samples displayed a serious loss of the target sequences during the lyophilisation and/or reconstitution steps. On the other hand, results on the RM03a prototype demonstrated that this loss can be largely abolished using an appropriate additive such as the sucrose used in this case. Additional tests performed to investigate the reason of these weak signals revealed that using such low DNA concentrations, a large proportion of plasmids had been lost already due to the attachment of the target DNA sequences to the inner wall of the polypropylene vials used (data not shown), which has been reported by Gaillard C, et al. previously [80]. Lyophilisation with an appropriate additive (e.g. 10\% sucrose) was found to reduce also this phenomenon.

Despite the successful development of the CRMGEN-FV RMs, these plasmidic RMs could not be processed as a set of candidate CRMs due to IPR issues: Although a preliminary agreement on these FV RMs was made with Prof. Dr. Rogier M. Bertina (LUMC), who was the main inventor patent holder of the human coagulation Factor V Leiden mutation, in the meantime Roche has bought the patent rights from Leiden University, which resulted in issues for finalisation of the development and release. On the other hand, the experiences and knowledge gained from the stability and homogeneity studies on these prototype RMs have been utilised in development and production of further clinical genetic (C)RMs. 
In summary, results of the FP5 CRMGEN project clearly demonstrated that it should be possible to create a (C)RM for any genetic test, for which the sequence variant is well characterised. On the other hand, the suitability of a certain type of genetic RM strongly depends on the kind of the sequence variant of interest. For example, PCR-based approach is not suited for triplet repeat diseases due to the instability of the triplet repeats during amplification. The advantages of short DNA fragment (i.e. PCR products or rDNA) type RMs include easy and economical production in large quantities, high reproducibility, versatility, and applicability for qualitative analysis. From another point of view, these genetic RMs cannot be used to assess the quality of DNA extraction procedures and contain only one defined specific sequence, which restricts the commutability and limits the wider use of such RMs. Assays for defined point mutations, such as the G20210A mutation in the prothrombin gene, usually amplify short fragments, and thus the problem can be tackled by appropriate selection of the reference sequence. However, the lack of other genomic sequences still limits the applicability of such RMs in multiplex techniques, including assays with internal amplification controls and might prevent the recognition of possible interferences arising from similar sequences in the genome. In addition, such RMs based on isolated gene sequences are particularly affected by gene patents, while cell lines or gDNA-based RMs might bypass this issue. Clearly, a very significant barrier of standardisation appears, if a private company is able to obtain a patent on a particular gene. Such patents have strong impact on the molecular genetic diagnostics and on the standardisation process of the genetic testing services [7,55,56]. For instance, they might inhibit the development and use of (C)RMs or constrain testing technologies moreover to prescribe standard testing procedures without traceability and adequate QC or the hindrance to the establishment of EQA schemes [7]. Even if we used non-proprietary vectors where applicable, gene patents were the main obstacles standing in the way of the further development and possible certification of two CRMGEN RMs (i.e. CRMGEN-HH and CRMGEN-FV), while the IFCC/IRMM Factor II G20210A mutation CRMs are not marketable in the USA without agreement with the patent holder.

Lyophilisation turned out to be a practical and effective tool for preservation of nucleic acid RMs. However, solid content and components of the material determine its behaviour during the freezedrying process. If the solid content is small $(\leq 2 \%)$, the structure of the product is mechanically unstable and particles of the material might not adhere to the matrix [81]. In such cases, water vapour can move these loose particles during the sublimation phase and deposit them somewhere in the vial or even transport them to the vacuum chamber resulting in loss of the compound of interest and dramatically increasing the contamination risk. Therefore, it is preferable to add substances that protect the DNA and form a stable structure, particularly in the field of clinical genetic RMs, where avoiding of any contamination is crucial. In addition, polyhydroxy compounds such as sugars (e.g. 
trehalose, sucrose, etc.) might serve as lyoprotectants and aid preservation forming a 'glass' structure, thus protecting the DNA from the breakdown of its helical structure and from oxidation during the freeze-drying process [76,81].

From another point of view, the observed shift of the melting peaks on the candidate NIBSC-WHO reference panel samples highlighted another issue: Several methods, such as the LightCycler [82] and the Taqman allelic discrimination [83] techniques, distinguish the different alleles on the basis of their characteristic melting temperatures with the hybridisation/Taqman probes. Sequence variants resulting in a mismatch between the probe and the target sequence lead to a decrease of ca. $2-10{ }^{\circ} \mathrm{C}$ in the Tm. The shift of the melting peaks on the WHO-NIBSC RMs was very close or even reached this range; however, the typical separation between the melting peaks on the heterozygous samples was maintained [84]. Such a pronounced shift in the Tm may affect also the sensitivity, specificity and efficiency of every PCR reaction, which might lead to biased or false genotyping results (e.g. allele-specific PCR, melting analysis) or quantification. Therefore, in order to avoid similar shortcomings, additives and presentation formats should be carefully selected and investigated for their effect on the test results.

In the frame of the present projects, many lyo- and/or cryoprotectants as well as other preservatives were screened for possible interferences. These experiments showed that some of the additives widely used to enhance the stability of biologic materials might interfere with the molecular diagnostic techniques directly (e.g. glycerol, Na-ascorbate, SDS) or after a given time period, when the material was stored in liquid/frozen format or following an inadequate freeze-drying resulting in high ( $>1 \%)$ moisture content. This latter observation not only emphasizes the pivotal importance of a proper lyophilisation protocol, but might be a possible explanation for the Tm shift on the WHONIBSC reference panel samples as well. On the other hand, our results showed that an acceptable shelf life of several years can be achieved even without freeze-drying in frozen aqueous solution at $>10^{8}$ copies of plasmid DNA using alkaline conditions ( $\mathrm{pH}$ 8.0-8.5) in Tris-EDTA buffer. Freezethaw tolerance of plasmids was also sufficient even without additives, which is concordant with the literature data [76,85]. In the case of RMs presented as PCR products or plasmids, this presentation format can reduce also the contamination risk, which is significantly higher in FD format.

Whilst the studies discussed above aimed to investigate and compare the suitability of different types of genetic RMs and to optimize the preservation of nucleic acid based RMs, the ultimate goal of the projects was the development and certification of CRMs for molecular genetic tests as well as to demonstrate their fitness for purpose and applicability in the quality assurance of clinical genetic testing [I,II,VI].

In co-operation with the IFCC C-MBT, two plasmids containing a 609-bp fragment of either the wild-type 20210G (named pIRMM-0001) or the 20210A (named pIRMM-0002) sequence variant 
of the human prothrombin gene were used to generate a set of 3 plasmid-type RMs corresponding to the wild-type, mutant, and heterozygous genotypes of the G20210A variant [I,II,III,IV,V].

The sequencing confirmed that the Factor II gene inserts of the RMs produced are identical to the GenBank reference sequence, except for the G20210A variant and an A20054G alteration in the mutated fragment. This second mutation was linked to the mutated sequence and its presence in the original patient sample means that it was not introduced during the production. Although the A20054G variant did not cause any problems during production of the plasmid-type RMs or in the field study, difficulties may arise when a primer is used that binds with its 3' end to this sequence. Thus, this mutation is reported in the documentation of the plasmidic CRMs [I,II,III,IV,V].

In the absence of any reference method for genetic testing, CRMs have to be evaluated by a consensus of recognised reference laboratories. Test compatibility and commutability of such plasmids as candidate CRMs was demonstrated in a study involving 13 expert laboratories using 9 different PCR-based methods [I]. However, the stability on the long-term of the freeze-dried plasmids (at $5 \times 10^{4} \mathrm{cp} /$ vial with BSA as additive) could not be confirmed, therefore the presentation format and storage conditions had been re-investigated and second batches have been processed as liquid frozen material in Tris-EDTA buffer (10 mM : $1 \mathrm{mM}$; $\mathrm{pH}$ 8.0) utilizing the results of the feasibility study on the preservation of plasmidic DNA. These RMs were certified after careful assessment of their stability, homogeneity and fitness for purpose in a field trial and are now available from the IRMM (http://www.irmm.jrc.be) [II,III,IV,V].

The CRMs IRMM/IFCC-490 (G20210/G20210 wild-type), IRMM/IFCC-491 (20210A/20210A homozygous mutant) and IRMM/IFCC-492 (G20210/20210A heterozygous mutant) have been the first clinical genetic CRMs introduced worldwide, which can be used for validation and harmonisation of PCR-based methods used for the detection of the G20210A sequence variant in the human prothrombin gene. Due to the lack of established reference methods in genetic testing, such CRMs play a crucial role to fulfil the traceability requirement as formulated in the European IVD Directive 98/79/EC [12]. In addition, any laboratories can use these CRMs as QC samples, which is particularly important for the rare homozygous mutant 20210A genotype [VI].

Although the commutability of plasmid type RMs is in principle restricted due to the limited length of the gene insert, this issue could be tackled by the appropriate selection of the reference sequence. All of the genotyping techniques applied in the field studies or in the proficiency testing exercise representing more than 50 different analytical procedures - were able to amplify the target sequence and to detect of the mutation of interest from the plasmids, proving that these CRMs are fit for purpose [I,II,III,IV,V,VI]. Furthermore, none of the laboratories reported atypical results (e.g. shifts in the melting temperature or allele discrimination plot, etc.) on the G20210 wild-type and 20210A mutant reference plasmids [I,VI]. 
On the other hand, short DNA fragment based RMs can be described and quantified more precisely, which was a key advantage during the characterisation studies. For example, although two other sets of QCMs are also available to assess the performance of test systems for the analysis of the prothrombin G20210A variant [67,86,V], their homogeneity and long term stability have not been demonstrated clearly as required by the relevant ISO standards for RMs [26,31,34]. Even though these RMs are to be used for qualitative analysis, the assessment of their homogeneity and stability on the long-term have to be confirmed by quantitative techniques [V,26,87].

Despite some apparent shortcomings of the plasmidic RMs discussed above, these data and achievements have proven that the strategy applied for the development and production of DNAbased CRMs containing defined sequence variants seems well suited and plasmid-type RMs can be successfully used in the field of genetic testing for the analysis of SNPs.

The paucity of the mutation-positive clinical samples for rare sequence variants, haplotypes or complex genotypes and the ethical issues related to their isolation from human sources pose a significant barrier to the development of RMs for some analytically challenging genotypes and contribute to the high error rates in the field of genetic testing $[7,88]$. However, plasmidic RMs containing a reference sequence of a human gene can be modified using the site-directed mutagenesis technique, which allows the generation of a defined mutation at the designated nucleotide position [89]. This approach had been successfully applied, when a SNP (i.e. C20209T) or a complex variation (i.e. [T20175G;20179_20180delAC]) were introduced into the human FII gene fragment of the wild-type reference plasmid pIRMM-0001 allowing the design of QCMs with rare genotypes/mutations, which were later used in a European proficiency testing exercise [VI].

Overall, the results of this interlaboratory comparison showed expected rates of genotyping failures on the wild-type and G20210A mutation samples without additional mutations. This error rate on the detection of SNPs corresponded to the latest data in the literature $[4,5,46,47]$ and may result in thousands of misclassifications yearly in the participant laboratories, which absolutely emphasizes the need for more detailed scrutiny to identify the sources of errors and to eliminate weaknesses. Such an evaluation of data was carried out using the method specifications and the raw genotyping results, which are rarely requested from the participants in the EQA schemes. This analysis of the error sources elucidated that majority of failures could be avoided by improved training, careful selection and validation of the methods applied [VI]. These observations underlined the pivotal importance of the laboratory personnel skills and suggested that training may be an efficient tool to improve the quality of genotyping services [90,VI].

Incidence of rare sequence variants interfering with some genotyping techniques is relatively low [91]; however, they can be easily misinterpreted and lead to an even higher error rate. Although the G20210A mutation is a well characterised risk factor for venous thromboembolism, the 
consequences on the analysis results of these adjacent variants were rarely investigated. Such rare sequence alterations should be reported as variants of unknown clinical significance clearly distinct from the wild-type or the G20210A mutation alleles [92].

In previous studies, competence of testing laboratories to recognize and correctly report such sequence variants had not been challenged. Our ring trial revealed that where additional sequence variants resulted in unusual genotyping data and were therefore in principle detectable, only a fraction of the laboratories recognised and adequately reported unexpected SNPs [VI]. These observations called the attention of the participants as well as of the broader community to the proper assessment of unusual genotyping results. For instance, as a consequence of the results published, Roche Diagnostics recalled and modified the Factor II (Prothrombin) G20210A Kit for the LC 2.0 instruments, because its macro component erroneously identified the C20209T mutation as wild-type genotype instead of an unknown variant. Furthermore, the Reference Institute for Bioanalytics started to challenge the recognition of rare SNPs in their EQA schemes [93].

From another point of view, the reporting of unusual genotyping results in a collaborative study is of prime importance also for RM producers. The low rate of such feedbacks (e.g. only two laboratories from 14 participants using the affected LightCycler assay system reported the Tm shift in the collaborative study on the NIBSC-WHO Reference Panel for Prothrombin Mutation G20210A [67]) stresses the need for a more careful selection of participants for field studies, which cannot rely on the accreditation of the laboratories alone, but this competence can be challenged in proficiency testing exercises [VI].

Finally, data on the genotype controls applied by the participants display that the access of clinical laboratories to QC materials representing the homozygous mutant genotypes is rather limited. Interestingly, one of the participants showing eminent performance in the study employed also plasmidic QCMs for rare sequence variants.

In conclusion, different types and formats of DNA-based RMs for genetic testing were processed and characterised for their suitability for the QA of molecular diagnostic methods. Genomic DNA has proven to be a suitable type of RMs for various genotyping techniques; however, its certification according to the ISO Guides 30-35 has not yet been achieved. A set of plasmidic CRMs for the analysis of the G20210A mutation in the Factor II gene had been developed and produced, which is now available from the IRMM. In addition, using these reference plasmids and the site-directed mutagenesis technique, QCMs for rare sequence variants were prepared and employed in a European proficiency testing study. It could be shown that ISO compliant plasmid-type RMs containing the desired SNPs can be produced and successfully used for quality assurance in genetic testing. Because DNA testing uses the same techniques wherever it is applied, the results of these projects can serve as guide and pilot for the production $[78,88,94,95]$ and use $[26,96]$ of $(C) R M s$ in genetic testing. 


\section{MAIN SCIENTIFIC ACHIEVEMENTS OF THE STUDY}

The most important achievements of the present study can be highlighted as follows.

1) Several types and presentation formats of DNA-based RMs were processed and investigated for their suitability as clinical genetic (C)RMs.

2) Different possibilities for the preservation of purified DNA-based RMs were examined and optimised to ensure a reasonable stability.

3) For the assessment of the homogeneity and stability of prototype/candidate genetic RMs, analytical procedures were improved and new methods were developed.

4) A model system for certification of DNA-based RMs was established and protocols of the isochronous stability and homogeneity studies for certification of genetic RMs were optimised and described according to ISO Guide 34 and the BCR guidelines.

5) These protocols and the methodology for development and production of genetic (C)RMs can be utilised in DNA-based tests of all kinds and the knowledge generated might facilitate the development of further nucleic acid based (C)RMs.

6) Three plasmidic CRMs for the detection of the G20210A variant in the human coagulation Factor II gene, namely the IRMM/IFCC-490, -491 and -492, were developed, produced and certified according to the ISO Guides 30-35.

7) Commutability studies were designed, organised and carried out to demonstrate the fitness for purpose of the candidate Factor II CRMs.

8) These CRMs are available from the IRMM for the quality assurance of PCR-based methods and have been the first plasmid-type clinical genetic CRMs introduced worldwide.

9) Utilizing the Factor II reference plasmids and the site-directed mutagenesis technique, QCMs for the FII G20210A variant and neighbouring rare SNPs were developed, produced and successfully applied in a European proficiency testing study to identify weaknesses of molecular genetic testing laboratories and to challenge their competence to recognize and adequately report interfering sequence variants. 


\section{PERSONAL CONTRIBUTION}

My personal contribution to the projects presented and to the articles referred to in this thesis is as follows:

IPTS ESTO study

- collected and summarised background information on the general situation and quality issues of genetic testing in Europe mainly focusing on the RMs and reference measurement systems, regulations in the EU, ISO/EN standards including ISO Guide 30-35, guidelines and recommendations for genetic testing and the impact of gene patenting on the field.

- commented the different drafts of the study report [7] and participated to the writing of a review published in the G.I.T. Laboratory Journal [21].

FP5 CRMGEN SCA

- designed and processed the CRMGEN-HH, CRMGEN-FraX and CRMGEN-FV prototype RMs in cooperation with the clinical genetic laboratories.

- prepared the project plan for each prototype RM according to the ISO Guide 34

- developed and optimised the freeze-drying protocol and performed the lyophilisation of the RMs.

- carried out the stability and homogeneity studies on the CRMGEN-HH, CRMGEN-FraX and CRMGEN-FV prototype RMs.

- developed improved analytical methods for the analysis of the CRMGEN-HH and CRMGEN-FV prototype RMs and tested samples of the stability and homogeneity studies.

- validated and described these measurement procedures as required by the IRMM's QA system.

- analyzed the data on the stability and homogeneity studies, wrote analysis reports according to quality system of the IRMM as well as a summary report to the CRMGEN consortium.

- prepared the templates for study protocol and result form for CRMGEN field studies.

- a model system for certification of DNA-based RMs was established and protocols of the isochronous stability and homogeneity studies for certification of genetic RMs were optimised and described according to ISO Guide 34 and the BCR guidelines

NIBSC-WHO collaborative studies CS 246 and CS 262

- participated to the collaborative study CS 262 candidate Reference Panel for Prothrombin Mutation G20210A genotyping the samples according to the study protocol provided using different techniques and lots of the kits applied.

- reported the results of the CS 262 and observations on the samples to the NIBSC [67]

- analyzed the samples of the NIBSC-WHO Reference Panel for the Factor V Leiden Mutation provided in the frame of the collaborative study CS 246.

- prepared internal analysis reports on the CS 262 as well as on the results on the CS 246 samples. 
Exploratory Research Project "Feasibility study: Preservation of plasmidic DNA" - initiated the project, wrote the project proposal to the 'Exploratory Research 2005' call

- designed and carried out the experiments including the production of the plasmidic prototype RMs using different additives, evaluated the data

- designed and performed the experiments to clarify the origin of the Tm-shift observed on both sets of the NIBSC-WHO Reference Panel samples.

- documented the experiments according to quality system of the IRMM: wrote analysis reports on the individual experiments and prepared a summary report on the project.

Development and production of the CRMs IRMM/IFCC-490, -491 and 492

- carried out the homogeneity and short-term stability studies on the $1^{\text {st }}$ batch of the candidate CRMs using two different techniques (a conventional qualitative PCR method and an improved LightCycler assay)

- developed an improved real-time PCR method, which enabled the relative quantitation of the samples, validated and described this assay according to the IRMM's QA system.

- designed, organised and conducted a commutability study on the $1^{\text {st }}$ batch of the candidate CRMs involving 2 Hungarian laboratories, analyzed the data and wrote the manuscript on the study [I]. - participated in the planning of the $2^{\text {nd }}$ batch of the candidate CRMs, prepared the docs required. - produced new lots of the candidate CRMs (i.e. cultivated the transfected E. coli strains, isolated and purified the reference plasmids, determined the DNA content, prepared the stock solutions for the three genotypes and processed the candidate CRMs).

- carried out the homogeneity, short-term and 12 months long-term stability studies on the $2^{\text {nd }}$ batch of the candidate CRMs and compared the two lots of the candidate CRMs

- contributed to the organisation of the characterisation study and to the preparation of the certification reports of the CRMs IRMM/IFCC-490, -491, and -492 [II,III,IV] as well as to the writing of the paper [V].

\section{FP6 EuroGenTest NoE}

- contributed to the preparation of the project proposal.

\section{Proficiency testing exercise using QCMs containing rare sequence variants}

- initiated and organised the proficiency testing study in cooperation with 3 EQA organisations.

- introduced the new mutations into the wild-type FII reference plasmid using the site-directed mutagenesis technique, produced and characterised the QCMs containing rare sequence variants.

- analyzed the data, prepared and distributed the feedback to the participants.

- prepared a report for the EuroGenTest consortium, and wrote the manuscript [VI]. 


\section{SUMMARY}

Recent progress in molecular pathology and biotechnology has made DNA-based assays important tools in patient care. DNA tests allow the detection of genetic alterations responsible for inheritable disease conditions, higher risk of developing diseases or altered drug effects. These tests have a high impact on clinical decision-making and thousands of different tests are already in routine use. Thus, quality issues and standardisation of DNA tests are particularly important.

In order to achieve and ensure the highest level of quality and accuracy in laboratory medicine, reference measurement systems (RMSs) has to be established. Reference measurement systems are needed for producing useful and reliable measurement results in the routine diagnostic services as well as in science and technology, which are comparable among laboratories and ultimately traceable to measurement standards of the highest metrological level.

The three elements of RMSs are internationally accepted and commonly agreed reference measurement procedures (RMP), certified reference materials (CRMs) and reference laboratories possibly collaborating in a network. Successful implementation of such systems can be further enhanced by External Quality Assurance (EQA) programmes. At present, the lack of RMSs in molecular genetic testing means that there are limitations in benchmarking against which an IVD manufacturer or a laboratory can judge the performance of its assays.

Reference materials (RMs) and certified reference materials (CRMs) are key elements of such RMSs and are recognised as an excellent tool for checking analytical accuracy and are valuable in creating the necessary reference points in the development of comprehensive measurement systems. (C)RMs are used for method development and validation, calibration, statistical quality control within a laboratory and to assess the performance of laboratories in proficiency testing schemes. Although the use of appropriate RMs to validate test equipment or testing methods is an important part of any analytical testing system, currently only a few RMs for clinical genetic testing are available. Therefore, the European Commission, Joint Research Centre, Institute of Reference Materials and Measurements (IRMM) launched several projects to develop and produce (certified) reference materials for molecular genetic testing and to elaborate the methodology to produce (C)RMs for any molecular genetic test.

The first major initiative, the Certified Reference Materials for Molecular Genetic Testing (CRMGEN) project focused on the development of candidate RMs for molecular genetic testing of inherited disorders and genetic risk factors, such as hereditary haemochromatosis $(\mathrm{HH})$, fragile $\mathrm{X}$ syndrome (FraX), cystic fibrosis (CF) and the coagulation Factor V Leiden (G1691A) mutation. Four basic types of clinical genetic RMs were developed, produced and investigated in different presentation formats for their suitability for RMs: cultured cell lines, recombinant DNA (plasmids), enzymatically amplified DNA fragments (PCR products) and genomic DNA. The prototype RMs 
developed were characterised and subjected to extensive field trials in diagnostic molecular genetic testing laboratories.

In line with the CRMGEN project, the Scientific Committee of Molecular Biology Techniques in Clinical Chemistry (C-MBT) of the IFCC has initiated a joint project with the IRMM to develop and produce a set of plasmidic CRMs for the analysis of the human coagulation Factor II (FII, prothrombin) gene G20210A variant. The reference plasmids containing a 609-bp fragment (GenBank accession number M17262, nt 26302 to 26910) of either the wild-type 20210G or the 20210A sequence variant of the human prothrombin gene were used to generate a set of 3 plasmidtype RMs corresponding to the wild-type, mutant, and heterozygous genotypes of the G20210A variant. The fragment chosen spans all primer annealing sites published until today.

These candidate CRMs were characterised using quantitative real-time PCR techniques for homogeneity, stability, sequence identity and their fitness for purpose. The CRMs are homogeneous and stable at $-20^{\circ} \mathrm{C}$. The sequence of the insert was confirmed by bidirectional DNA sequence analysis. Each vial contains an indicative volume of $50 \mu \mathrm{L}$ corresponding to approximately $1 \mathrm{ng}$ of plasmid DNA. The CRMs IRMM/IFCC-490 (G20210/G20210 wild-type), IRMM/IFCC-491 (20210A/20210A homozygous mutant) and IRMM/IFCC-492 (G20210/20210A heterozygous mutant) have been the first clinical genetic CRMs introduced worldwide, which can be used for validation and harmonisation of PCR-based methods used for the detection of the G20210A sequence variant in the human Factor II gene.

Furthermore, the IRMM and the Department of Biochemistry of the University of Szeged agreed on a proficiency testing (PT) study in cooperation with 3 European EQA organisations. In the frame of this study, new mutations were introduced into the wild-type FII reference plasmid using sitedirected mutagenesis technique and the QCMs containing the modified and/or reference plasmids were distributed to the participants to assess how clinical laboratories interpret unusual genotyping results and how many of these laboratories recognize interfering mutations and report them correctly. Additional aims of the study were to test identify weaknesses of genetic testing services, to develop QCMs for rare sequence variants, and to gather more information about the requirements for DNA-based candidate RMs.

Using the plasmids containing rare sequence variants (i.e. the C20209T and the [T20175G; 20179_20180delAC] mutations) resulting in unusual results in some genotyping assays and the FII reference plasmids four QCMs were produced and distributed to the participants of the proficiency testing exercise.

After removing of duplications resulting from the overlaps of the EQA schemes, 189 laboratories from 21 countries participated to the study. These participants in the survey applied a large variety of genotyping techniques representing 50 different analytical procedures. Our findings revealed that 
DNA analysis for the detection of the prothrombin G20210A mutation is reliable in a majority of laboratories; however, some of the participating laboratories were not able to recognize and correctly interpret unusual genotyping results caused by rare SNPs. In this study, most of the false results arose from inadvertent errors by laboratory personnel and not from technical failure of the method as such and the results indicates that the majority of the failures could be avoided by improved training and careful selection and validation of the methods applied.

Furthermore, all of the genotyping techniques applied in the proficiency testing exercise representing more than 50 different analytical procedures - were able to amplify the target sequence and to detect of the mutation of interest from the plasmids. None of the laboratories reported atypical results (e.g. shifts in the melting temperature or allele discrimination plot, etc.) on the G20210 wild-type and 20210A mutant reference plasmids. These results proved that these CRMs are fit for purpose.

In conclusion, different types and formats of DNA-based RMs for genetic testing were processed and characterised for their suitability for the quality assurance of molecular diagnostic methods. Genomic DNA has proven to be a suitable type of RMs for various genotyping techniques; however, its certification according to the ISO Guides 30-35 has not yet been achieved. A set of plasmidic CRMs for the analysis of the G20210A mutation in the Factor II gene had been developed and produced, which is now available from the IRMM. In addition, using these reference plasmids and the site-directed mutagenesis technique, QCMs for rare sequence variants were prepared and employed in a European proficiency testing exercise. It could be shown that ISO compliant plasmid-type RMs containing defined SNPs can be produced and successfully applied for the QA of PCR-based molecular genetic techniques. Because DNA testing uses the same techniques wherever it is applied, these results and the knowledge gained from the projects can be utilised in the development of further DNA-based (C)RMs for any molecular genetic test and contribute to the quality improvement of genetic testing services. 


\section{REFERENCES}

1. Dequeker E, Cassiman JJ. Evaluation of CFTR gene mutation testing methods in 136 diagnostic laboratories: report of a large European external quality assessment. Eur J Hum Genet 6:165-75. (1998)

2. Losekoot M, Bakker B, Laccone F, Stenhouse S, Elles R. A European pilot quality assessment scheme for molecular diagnosis of Huntington's disease. Eur J Hum Genet 7:217-22. (1999)

3. Neumaier M, Braun A, Gessner R, Funke H. Experiences with external quality assessment (EQA) in molecular diagnostics in clinical laboratories in Germany. Clin Chem Lab Med 38:161-3. (2000)

4. Dequeker E, Ramsden S, Grody WW, Stenzel TT, Barton DE. Quality control in molecular genetic testing. Nat Rev Genet 2:717-23. (2001)

5. Tripodi A, Peyvandi F, Chantarangkul V, Menegatti M, Mannucci PM. Relatively poor performance of clinical laboratories for DNA analyses in the detection of two thrombophilic mutations - a cause for concern. Thromb Haemost 88:690-1. (2002)

6. Organisation for Economic Co-operation and Development (OECD) Genetic Testing: Policy issues for the new millennium. OECD Proceedings (2000)

7. Ibarreta D, Bock AK, Klein CL, Rodriguez-Cerezo E. Towards quality assurance and harmonisation of genetic testing services in the EU. Report EUR 20977 EN (2003)

8. Pinto-Basto J, Guimaraes B, Rantanen E, et al. Scope of definitions of genetic testing: evidence from a EuroGentest survey. J Community Genet 1:29-35. (2010)

9. OECD guidelines for quality assurance in molecular genetic testing. Organisation for Economic Co-operation and Development. (2007) http://www.oecd.org/dataoecd/43/6/38839788.pdf

10. Kristoffersson U. Regulatory Issues for Genetic Testing in Clinical Practice. Mol Biotechnol 40:113-117. (2008)

11. ECRI Institute Evidence-based Practice Center. Quality, Regulation and Clinical Utility of Laboratory-developed Molecular Tests - Technology Assessment Report. (2010) http://www.cms.gov/determinationprocess/downloads/id72TA.pdf

12. Directive 98/79/EC of the European Parliament and of the Council of 27 October 1998 on in vitro Diagnostic Medical Devices, Off J Eur Comm L331:1-37. (1998)

13. Müller MM. Implementation of Reference Systems in Laboratory Medicine. Clin Chem 46:1907-09. (2000)

14. Thienpont LM, Van Uytfanghe K, De Leenheer AP. Reference measurement systems in clinical chemistry. Clin Chim Acta 323:73-87. (2002) 
15. EN ISO 17511:2003 In vitro diagnostic medical devices - Measurement of quantities in biological samples - Metrological traceability of values assigned to calibrators and control materials. International Organization for Standardization, Geneva, Switzerland (2003)

16. Panteghini M. Traceability as a unique tool to improve standardization in laboratory medicine. Clin Biochem 42:236-40. (2009)

17. EN ISO 15193:2009, In vitro diagnostic medical devices - Measurement of quantities in samples of biological origin - Requirements for content and presentation of reference measurement procedures. International Organization for Standardization, Geneva, Switzerland (2009)

18. EN 12286:1998, In vitro diagnostic medical devices - Measurement of quantities in samples of biological origin - Presentation of reference measurement procedures. European Committee for Standardization, Brussels, Belgium (1998)

19. ISO/IEC Guide 99:2007 corrected in 2010 International vocabulary of metrology — Basic and general concepts and associated terms (VIM). International Organization for Standardization / International Electrotechnical Commission, Geneva, Switzerland (2007)

20. EN 14136:2004 Use of external quality assessment schemes in the assessment of the performance of in vitro diagnostic examination procedures. European Committee for Standardization, Brussels, Belgium (2004)

21. Schimmel H, Emons H, Klein CL, Márki-Zay J. Reference Materials as Quality Assurance Tools for Laboratory Diagnostics. G.I.T. Laboratory Journal 8;6:32-33. (2004)

22. Schimmel H, Zegers I, Emons H. Standardization of protein biomarker measurements: is it feasible? Scand J Clin Lab Invest Suppl 242:27-33. (2010)

23. http://cmgs.org/BPGs/Best Practice Guidelines.htm

24. http://www.eurogentest.org/professionals/documents/

25. http://www.emqn.org/emqn/Best+Practice/

26. Gancberg D, Corbisier P, Schimmel H, Emons H. Guidance document on the use of reference materials in genetic testing. Report EUR 23256 EN (2008)

27. Rousseau F, Gancberg D, Schimmel H, et al. Considerations for the development of a reference method for sequencing of haploid DNA - an opinion paper on behalf of the IFCC Committee on Molecular Diagnostics. Clin Chem Lab Med 47:1343-50. (2009)

28. Hill F, Gemünd C, Benes V, Ansorge W, Gibson TJ. An estimate of large-scale sequencing accuracy. EMBO Reports 1:29-31. (2000)

29. ISO Guide 30:1992/Amd 1:2008 Revision of definitions for reference material and certified reference material. International Organization for Standardization, Geneva, Switzerland (2008) 
30. Emons H, Fajgelj A, van der Veen AMH, Watters R. New definitions on reference materials. Accred Qual Assur 10:576-578. (2006)

31. ISO Guide 34:2009, General requirements for the competence of reference material producers, International Organization for Standardization, Geneva, Switzerland (2009)

32. Emons H. The "RM family"- Identification of all its members. Accred Qual Assur 10:690-691. (2006)

33. ISO Guide 31:2000, Reference materials - Contents of certificates and labels International Organization for Standardization. International Organization for Standardization, Geneva, Switzerland (2000)

34. ISO Guide 35:2006, Reference materials - General and statistical principles for certification. International Organization for Standardization, Geneva, Switzerland (2006)

35. Lamberty A, Schimmel H, Pauwels J. The study of the stability of reference materials by isochronous measurements. Fresenius J Anal Chem 360:359-61. (1998)

36. EN ISO 15194:2009, In vitro diagnostic medical devices - Measurement of quantities in samples of biological origin - Requirements for certified reference materials and the content of supporting documentation. International Organization for Standardization, Geneva, Switzerland (2009)

37. Vesper HW, Miller WG, Myers GL. Reference Materials and Commutability. Clin Biochem Rev 28:139-147. (2007)

38. IFCC External Quality Assessment scheme for Reference (calibration) Laboratories in Laboratory Medicine: IFCC-RELA-EQAS procedure manual. International Federation of Clinical Chemistry and Laboratory Medicine (2008)

http://www.dgkl-rfb.de:81/IFCC EQAS ProcManual.pdf

39. Siekmann L. Requirements for Reference (Calibration) Laboratories in Laboratory Medicine Clin Biochem Rev 28:149-154. (2007)

40. Panteghini M. Traceability, reference systems and result comparability. Clin Biochem Rev 28:97-104. (2007)

41. Rej R, Norton-Wenzel CS, Cao Z. Target values and method evaluation in proficiency testing programs. Clin Chem 47:2185-6. (2001)

42. EN ISO 15195:2003, Laboratory medicine. Requirements for reference measurement laboratories. International Organization for Standardization, Geneva, Switzerland (2003) 
43. Armbruster D, Miller RR. The Joint Committee for Traceability in Laboratory Medicine (JCTLM): A Global Approach to Promote the Standardisation of Clinical Laboratory Test Results. Clin Biochem Rev 28:105-114. (2007)

44. CLSI document EP14-A2. Evaluation of matrix effects; Approved Guideline - Second Edition. Clinical and Laboratory Standards Institute, Wayne, Pennsylvania, USA (2005)

45. http://www.eurogentest.org/professionals/databases/info/public/unit1/eqa/molecular genetics/eq a scheme provision 2005.xhtml

46. Jennings I, Kitchen S, Woods TA, Preston FE. Multilaboratory testing in thrombophilia through the United Kingdom National External Quality Assessment Scheme (Blood Coagulation) Quality Assurance Program. Semin Thromb Haemost 31:66-72. (2005)

47. Tripodi A, Chantarangkul V, Menegatti M, Tagliabue L, Peyvandi F. Performance of clinical laboratories for DNA analyses to detect thrombophilia mutations. Clin Chem 51:1310-1. (2005)

48. Ahmad-Nejad P, Dorn-Beineke A, Pfeiffer U, et al. Methodologic European external quality assurance for DNA sequencing: the EQUALseq program. Clin Chem 52:716 -27. (2006)

49. ISO/IEC Guide 98-3:2008, Uncertainty of measurement - Part 3: Guide to the expression of uncertainty in measurement (GUM:1995) International Organization for Standardization, Geneva, Switzerland (2008)

50. CLSI document MM1-A2. Molecular Diagnostic Methods for Genetic Diseases; Approved Guideline - Second Edition. Clinical and Laboratory Standards Institute, Wayne, Pennsylvania, USA (2006)

51. Barker SD, Bale S, Booker J, et al. Development and characterization of reference materials for MTHFR, SERPINA1, RET, BRCA1, and BRCA2 genetic testing. J Mol Diagn 11:553-61. (2009)

52. Pratt VM, Zehnbauer B, Wilson JA, et al. Characterization of 107 genomic DNA reference materials for CYP2D6, CYP2C19, CYP2C9, VKORC1, and UGT1A1: a GeT-RM and Association for Molecular Pathology collaborative project. J Mol Diagn 12:835-46. (2010)

53. Pauwels J, Schimmel H, Lamberty A. Criteria for the certification of internationally acceptable reference materials. Clin Biochem 31:437-9. (1998)

54. Directive 98/44/EC of the European Parliament and of the Council of 6 July 1998 on the legal protection of biotechnological inventions. Off J Eur Comm L 213:13-21. (1998)

55. Merz JF, Kriss AG, Leonard DG, Cho MK. Diagnostic testing fails the test. Nature. 415:577-9. (2002) 
56. Soini S, Aymé S, Matthijs G. Public and Professional Policy Committee and Patenting and Licensing Committee. Patenting and licensing in genetic testing: ethical, legal, and social issues. Eur J Hum Genet 16 Suppl 1:S10-50. (2008)

57. King C, Barton DE. Best practice guidelines for the molecular genetic diagnosis of Type 1 (HFE-related) hereditary haemochromatosis. BMC Med Genet 7:81. (2006)

58. Dequeker E, Stuhrmann M, Morris MA, et al. Best practice guidelines for molecular genetic diagnosis of cystic fibrosis and CFTR-related disorders--updated European recommendations. Eur J Hum Genet 17:51-65. (2009)

59. Biancalana V, Steinbach P, Stenhouse S. Draft Best Practice Guidelines for Molecular Analysis in Fragile X Syndrome. The European Molecular Genetics Quality Network (EMQN), Strassbourg, France (2006)

60. Bertina RM, Koeleman BP, Koster T, et al. Mutation in blood coagulation Factor V associated with resistance to activated protein C. Nature 369:64-7. (1994)

61. Poort SR, Rosendaal FR, Reitsma PH, Bertina RM. A common genetic variation in the 3'untranslated region of the prothrombin gene is associated with elevated plasma prothrombin levels and an increase in venous thrombosis. Blood 88:3698-3703. (1996)

62. McGlennen RC, Key NS. Clinical and Laboratory Management of the Prothrombin G20210A Mutation. Arch Pathol Lab Med 126:1319-1325. (2002)

63. Cassiman JJ. Research network: EuroGentest - a European Network of Excellence aimed at harmonizing genetic testing services. Eur J Hum Genet 13:1103-05. (2005)

64. http://www.nibsc.ac.uk/documents/ifu/04-224.pdf

65. Gray E, Hawkins JR, Morrison M, et al. Establishment of the 1st International Genetic Reference Panel for Factor V Leiden, human gDNA. Thromb Haemost 96:215-9. (2006)

66. http://www.nibsc.ac.uk/documents/ifu/05-130.pdf

67. Gray E, Metcalfe P. A collaborative study on the proposed 1st International Genetic Reference Panel for Prothrombin Mutation (G20210A), human gDNA (05/130), WHO (2005) http://www.who.int/bloodproducts/cs/052022.pdf

68. van der Veen, A.M.H., Linsinger, T.P.J., Lamberty, A., Pauwels, J. Uncertainty calculations in the certification of reference materials 3. Stability study. Accred Qual Assur 6:257-263. (2001) 69. van der Veen, A.M.H., Linsinger, T.P.J., Pauwels, J. Uncertainty calculations in the certification of reference materials. 2. Homogeneity study. Accred Qual Assur 6:26-30. (2001) 
70. Stott MK, Fellowes AP, Upton JD, Burt MJ, George PM. Simple multiplex PCR for the simultaneous detection of the C282Y and H63D hemochromatosis (HFE) gene mutations. Clin Chem 45:426-8. (1999)

71. Meadows CA, et al. Simultaneous Detection of C282Y and H63D Hemochromatosis Mutations Using LCRed 640 and LCRed 705 Labelled Hybridization Probes. In: Meuer S, Wittwer CT, Nakagawara K. (Eds.) Rapid Cycle Real-Time PCR - Methods and Applications Springer-Verlag Berlin, pp 127-134. (2001)

72. Biancalana V, Macpherson J. Fragile X disease. in Molecular Diagnosis of Genetic Diseases, Second Edition, Ed: R. Elles and R. Mountford, Humana Press, Totowa, NJ, pp 157-182. (2004)

73. Laboratory Biosafety Manual. Third Edition. World Health Organisation, Geneva, Switzerland (2004) http://www.who.int/csr/resources/publications/biosafety/Biosafety7.pdf

74. Barton D, Klein CL, Stacey G. Certified reference materials for genetic testing. In: Fuchs J, Podda M, editors. Encyclopedia of diagnostic genomics and proteomics. New York: Marcel Dekker, pp 226-31. (2005)

75. Evans RK, Xu Z, Bohannon KE, Wang B, Bruner MW, Volkin DB. Evaluation of degradation pathways for plasmid DNA in pharmaceutical formulations via accelerated stability studies. J Pharm Sci 89:76-87. (2000)

76. Anchordoquy TJ, Allison SD, Molina MC, Girouard LG, Carson TK. Physical stabilization of DNA-based therapeutics. Drug Discov Today 6:463-470. (2001)

77. Pratt VM, Caggana M, Bridges C, et al. Development of genomic reference materials for cystic fibrosis genetic testing. J Mol Diagn 11:186-93. (2009)

78. Hawkins M, Boyle J, Wright KE, et al. Preparation and validation of the first WHO international genetic reference panel for Fragile X syndrome. Eur J Hum Genet 19:10-7. (2011)

79. https://www-s.nist.gov/srmors/certificates/view certPDF.cfm?certificate=2399

80. Gaillard C, Strauss F. Avoiding adsorption of DNA to polypropylene tubes and denaturation of short DNA fragments. Technical Tips Online 3:63-65. (1998)

81. Rey L, May JC (Eds). Freeze-drying/lyophilization of pharmaceutical and biological products. $2^{\text {nd }}$ Ed. Marcel Dekker Inc., New York, USA (2004)

82. Wittwer CT, Ririe KM, Andrew RV, David DA, Gundry RA, Balis UJ. The LightCycler: a microvolume multisample fluorimeter with rapid temperature control. Biotechniques 22:176-81. (1997)

83. Livak KJ. Allelic discrimination using fluorogenic probes and the 5 ' nuclease assay. Genet Anal 14:143-9. (1999) 
84. Lyon E, Wittwer CT. LightCycler technology in molecular diagnostics. J Mol Diagn 11:93-101. (2009)

85. Rossmanith P, Röder B, Frühwirth K, Vogl C, Wagner M. Mechanisms of degradation of DNA standards for calibration function during storage. Appl Microbiol Biotechnol 89:407-17. (2011)

86. INTROL ${ }^{\mathrm{TM}}$ Thrombosis Genotype Panel, product of the Maine Molecular Quality Control Inc. http://www.mmqci.com/qc-g123.php

87. Payne DA, Mamotte CD, Gancberg D, et al. IFCC Committee for Molecular Diagnostics (CMD). Nucleic acid reference materials (NARMs): definitions and issues. Clin Chem Lab Med 48:1531-5. (2010)

88. Chen B, O' Connell CD, Boone DJ, et al. Developing a sustainable process to provide quality control materials for genetic testing. Genet Med 7:534-49. (2005)

89. Jarvis M, Iyer RK, Williams LO, et al. A novel method for creating artificial mutant samples for performance evaluation and quality control in clinical molecular genetics. J Mol Diagn 7:247-51. (2005)

90. Dorn-Beineke A, Ahmad-Nejad P, Pfeiffer U, et al. Improvement of technical and analytical performance in DNA sequencing by external quality assessment-based molecular training. Clin Chem 52:2072-8. (2006)

91. Lyon E. Discovering rare variants by use of melting temperature shifts seen in melting curve analysis [Editorial]. Clin Chem 51:1331-2. (2005)

92. Richards CS, Bale S, Bellissimo DB, et al; Molecular Subcommittee of the ACMG Laboratory Quality Assurance Committee. ACMG recommendations for standards for interpretation and reporting of sequence variations: Revisions 2007. Genet Med 10:294-300. (2008)

93. Ahmad-Nejad P. Quality management and proficiency testing in molecular diagnostics. $55^{\text {th }}$ Congress of the Hungarian Society of Laboratory Medicine. 26-28. August 2010. Pécs, Hungary

94. Berwouts S, Gordon JT, Rundell CA, Barton DE, Dequeker E. Evaluation and use of a synthetic quality control material, included in the European external quality assessment scheme for cystic fibrosis. Hum Mutat 29:1063-70. (2008)

95. Boyle J, Hawkins M, Barton DE, et al. Establishment of the first WHO international genetic reference panel for Prader Willi and Angelman syndromes. Eur J Hum Genet 19:857-64. (2011)

96. Berwouts S, Christensen TM, Brandon J, et al. Multiallelic Synthetic Quality Control Material: Lessons Learned from the Cystic Fibrosis External Quality Assessment Scheme. Genet Test Mol Biomarkers. 2011 Apr 10. [Epub ahead of print] 
I. 


\section{Reference materials (RMs) for analysis of the human factor II (prothrombin) gene G20210A mutation}

\author{
Christoph L. Klein ${ }^{1, *}$, János Márki-Zay ${ }^{1}$, \\ Philippe Corbisier ${ }^{1}$, David Gancberg ${ }^{1}$, Susan \\ Cooper $^{2}$, Donato Gemmati ${ }^{3}$, Walter-Michael \\ Halbmayer ${ }^{4}$, Steve Kitchen ${ }^{2}$, Béla Melegh ${ }^{5}$, \\ Michael Neumaier ${ }^{6}$, Johannes Oldenburg ${ }^{7}$, \\ Elisabeth Oppliger Leibundgut ${ }^{8}$, Pieter $\mathrm{H}$. \\ Reitsma ${ }^{9}$, Sandra Rieger ${ }^{10}$, Heinz G.
}

Schimmel ${ }^{1}$, Michael Spannagl ${ }^{11}$, Attila Tordai ${ }^{12}$, Alberto Tosetto ${ }^{13}$, Sophie Visvikis ${ }^{14}$, Renata Zadro $^{15}$ and Christine Mannhalter ${ }^{10, * *}$

${ }^{1}$ Institute for Reference Materials and Measurements, European Commission, Joint Research Centre, Geel, Belgium

${ }^{2}$ Department of Coagulation, Sheffield Haemophilia and Thrombosis Centre, Royal Hallamshire Hospital, Sheffield, United Kingdom

${ }^{3}$ Haemostasis and Thrombosis Study Centre, University of Ferrara, Ferrara, Italy

${ }^{4}$ Central Laboratory, Municipal Hospital Vienna-

Lainz, Vienna, Austria

${ }^{5}$ Department of Medical Genetics and Child Development, University of Pécs, Pécs, Hungary ${ }^{6}$ Institute for Clinical Chemistry, University Hospital Mannheim, University of Heidelberg, Germany ${ }^{7}$ Institute of Transfusion Medicine and Immunohaematology, DRK Blood Donor Service Baden-Wurttemberg/Hessen, Frankfurt am Main, Germany

${ }^{8}$ Laboratory of Molecular Diagnostics, Division of Hematology, University Hospital of Berne, Berne, Switzerland

${ }^{9}$ Laboratory for Experimental Internal Medicine, Academic Medical Centre, University of Amsterdam, Amsterdam, The Netherlands

${ }^{10}$ Clinical Institute of Medical and Chemical Laboratory Diagnostics, Medical University Vienna, Vienna, Austria

${ }^{11}$ Haemostasis Division, University Hospital Munich, Germany

*Corresponding author: Christoph L. Klein, European Commission, Joint Research Centre, Institute for Reference Materials and Measurements, Retieseweg 111, 2440 Geel, Belgium

Phone: +32-14-571634, Fax: +32-14-571548,

E-mail: christoph.klein@cec.eu.int

${ }^{*} \mathrm{CM}$ is the past chairperson of the Committee of Molecular Biology Techniques in Clinical Chemistry (C-MBT) of the International Federation of Clinical Chemistry and Laboratory Medicine (IFCC). CM initiated the project for production of a certified reference material using the prothrombin G20210A mutation as a model system, together with members of C-MBT and on behalf of the IFCC. The Scientific Division of IFCC supported the project ideally and by financial funds. The manuscript has been approved by members of the Scientific Division.
12 Laboratory of Molecular Genetics, National Medical Centre, Institute of Hematology and Immunology, Budapest, Hungary

${ }^{13}$ Hematology Department, S. Bortolo Hospital, Vicenza, Italy

${ }^{14}$ INSERM U525 Faculté de Pharmacie, Nancy,

France

${ }^{15}$ Clinical Institute of Laboratory Diagnosis, University School of Medicine and Clinical Hospital Centre Zagreb, Zagreb, Croatia

\section{Abstract}

The Scientific Committee of Molecular Biology Techniques (C-MBT) in Clinical Chemistry of the IFCC has initiated a joint project in co-operation with the European Commission, Joint Research Centre, Institute of Reference Materials and Measurements to develop and produce plasmid-type reference materials (RMs) for the analysis of the human prothrombin gene G20210A mutation. Although DNA tests have a high impact on clinical decision-making and the number of tests performed in diagnostic laboratories is high, issues of quality and quality assurance exist, and currently only a few RMs for clinical genetic testing are available. A gene fragment chosen was produced that spans all primer annealing sites published to date. Both the wild-type and mutant alleles of this gene fragment were cloned into a pUC18 plasmid and two plasmid RMs were produced. In addition, a mixture of both plasmids was produced to mimic the heterozygous genotype. The present study describes the performance of these reference materials in a commutability study, in which they were tested by nine different methods in 13 expert laboratories. This series of plasmid RMs are, to the best of our knowledge, the first plasmid-type clinical genetic RMs introduced worldwide.

Keywords: metrological traceability; molecular diagnostic testing; mutation; polymerase chain reaction; prothrombin; recombinant DNA; reference standards.

\section{Introduction}

Recent progress in molecular pathology and biotechnology has made DNA-based assays important tools in patient care. DNA tests allow the detection of genetic alterations responsible for inheritable disease conditions, higher risk of developing diseases or altered drug effects. These tests are increasingly used for diagnosis, risk stratification and choice of treatment for patients. DNA test results can be predictive 
for future onset of disorders prior to the manifestation of clinical symptoms, and thus have significant implications for the individual patient and the sibship. Often, testing is only carried out once in a lifetime, and therefore, incorrect genotyping results can have dramatic consequences for the person tested. Thus, quality issues and standardisation of DNA tests are particularly important. Depending on the system used, relatively high error rates for clinical genetic testing have been reported (1-4), specifically including thrombophilic mutations, such as the prothrombin G20210A mutation $(5,6)$. To overcome these issues, the quality of testing has to be assured and improved.

Certified reference materials (CRMs) are recognised as an excellent tool for checking analytical accuracy and are valuable in creating crucial reference points in the development of comprehensive measurement systems (7). CRMs are needed to achieve traceability and comparability of test results and harmonisation of measurement systems. Suitable reference materials (RMs) are widely used as tools for quality assurance (QA). The in vitro Diagnostic Medical Device (IVD) Directive of the EC $(98 / 79 / E C)$ (8) has been in force since December 2003. It requires IVD manufacturers to assure the traceability of values assigned to calibrators and control materials through available reference measurement procedures and/or available reference materials of a higher order. The current lack of CRMs for molecular genetic tests leads to assays not traceable to an accepted common standard. Therefore, the Scientific Committee of Molecular Biology Techniques in Clinical Chemistry (C-MBT) of the International Federation of Clinical Chemistry and Laboratory Medicine (IFCC) in co-operation with the European Commission, Joint Research Centre, Institute for Reference Materials and Measurements (IRMM) initiated a joint project to develop and produce RMs for the analysis of the human prothrombin gene G20210A mutation. In parallel, batches of the described RMs were produced as candidate CRMs, which will be provided as frozen material. These are currently undergoing an IRMM certification process. These clinical genetic RMs are among the first RMs introduced worldwide in this area. Its characteristics and performance in a collaborative field trial are described and discussed in the present article.

\section{Background}

\section{Selection of prothrombin G20210A mutation as target}

The G20210A mutation in the factor II (prothrombin) gene was discovered in 1996 by Poort et al. (9). It is due to a $G \rightarrow$ A transition at base pair 20210 in the $3^{\prime}$ untranslated region of the prothrombin gene. The heterozygous mutation occurs with a frequency of approximately $2-5 \%$ in the Caucasian population. Carriers of this mutation have significantly higher mean plasma prothrombin levels (9). Since the first publication of the G20210A mutation, it has been demonstrated in a number of publications that this genetic variant is a significant and independent risk factor for venous thromboembolism (VTE) (10). The clinical significance of this mutation, the indications for testing and the therapeutic consequences have been elucidated (11).

Analysis of this single nucleotide polymorphism (SNP) is one of the most commonly applied genetic tests in clinical laboratories. Several methods are used for testing, and commercial kits are increasingly applied.

\section{Formats of clinical genetic RMs}

There are many possible formats of genetic RMs, including CRMs: cell lines, genomic DNA, recombinant DNA, synthetic DNA or PCR products, all differing from typical clinical material for investigation. In the present study, RMs for QA were produced as freeze-dried plasmid materials. The advantages of plasmid-type RMs are easy and economical production in large quantities, high reproducibility, versatility, and applicability for qualitative analysis. The main drawback of these genetic RMs is that they cannot be used to assess the quality of DNA extraction procedures. In addition, the length of the fragments inserted is limited and commutability will be impaired if such RMs are applied to methods that use primers outside of the fragment sequence. RMs must have a reasonable shelf life to be useful. Therefore, RM stability is of central importance and has to be guaranteed by the producer (12).

\section{Materials and methods}

\section{Origin of extracted DNA}

The prothrombin gene fragment was selected in the Molecular Biology Division, Department of Clinical Chemistry and Laboratory Medicine, Medical University Vienna, Vienna, Austria. To achieve the highest possible level of test compatibility and commutability, the fragment chosen spans all primer annealing sites published to date (GenBank accession number M17262, nt 26302-26910). Using suitable primers (Pro-fw, 5'-gCACAgACggCTgTTCTCTT-3'; and Pro-rev, 5'-CCCgAgTgCTCggACTACCA-3', synthesised by VBC Genomics, Vienna, Austria, HPLC-purified) a PCR fragment was generated from coded samples of individuals previously tested by other methods and known to carry no mutation or to carry the $G \rightarrow A$ mutation at position 20210 (nt 26784 in the GenBank sequence), respectively.

The individuals had given informed consent to genetic analysis. The use of coded preserved DNA for means of quality assurance conforms to the current Austrian and European regulations.

\section{Cloning of the factor II gene fragment and processing of the stock plasmids}

After initial expansion and characterisation in a pCR2.1 vector (TA Cloning Kit, Invitrogen $\mathrm{GmbH}$, Lofer, Austria), the prothrombin gene fragments were amplified with the following primers: F2cloning-fw, 5'-CgAgCTCggATCCACTAgTAA3'; and F2cloning-rev, 5'-gCCAgTgTgATggATATCTgC-3' 
(Proligo Biochemie GmbH, Hamburg, Germany) and subcloned into the non-patented pUC18 plasmid using the Clontech AdvanTAge PCR Cloning Kit (BD Biosciences Benelux N.V., Erembodegem, Belgium) according to the instructions of the manufacturers.

The resulting new plasmids were called pIRMM-0001 and pIRMM-0002, containing the wild-type or mutant sequence of the factor II gene fragment, respectively. These plasmids were introduced into and amplified in the TOP10 competent E. coli strain provided in the cloning kit.

\section{Amplification and production of stock plasmids}

Streaks of bacterial colonies were tested by PCR for the presence of the inserts. The orientation of the inserts was assessed by digestion with Hindlll. A single clone containing the wild-type or mutant fragment, respectively, in the same orientation was selected and used as the source of the plasmid stocks for the CRMs.

Plasmid stocks were obtained after amplification overnight in liquid LB broth medium (LB Ampicillin-100 Broth; Fluka, Sigma, Bornem, Belgium) containing $100 \mu \mathrm{g} / \mathrm{mL}$ ampicillin and purification using a Qiagen Plasmid Maxi Kit (Qiagen, Westburg, Leusden, Netherlands).

\section{DNA quantitation}

The stock solution (Tris-HCl) of plasmid DNA was quantified using the PicoGreen ${ }^{\circledR}$ dsDNA quantitation kit (Molecular Probes, VWR International, Leuven, Belgium) according to the manufacturer's instructions. The copy number of the plasmids in the stock solution was calculated on the basis of DNA concentration.

\section{Sequencing of the stock plasmids}

The factor II gene inserts in the stock plasmids were sequenced for confirmation using the primers (Pro-fw and Pro-rev) described above and CEO Dye Terminator Cycle Sequencing Kit with the Beckman CEO2000XL Sequencer (Beckman Coulter Inc.) according to the manufacturer's instructions.

\section{Production of pIRMM-0001, -0002 and mixed RMs}

Sterile, self-standing, high-recovery $1.5-\mathrm{mL}$ polypropylene vials were filled with either $5 \times 10^{4}$ copies of plasmid pIRMM$0001,5 \times 10^{4}$ copies of pIRMM-0002 or a mixture of $2.5 \times 10^{4}$ copies of pIRMM-0001 and $2.5 \times 10^{4}$ copies of pIRMM-0002 in a volume of $100 \mu \mathrm{L}$ containing $100 \mu \mathrm{g} / \mathrm{mL}$ bovine serum albumin (BSA) as additive. These samples were freeze-dried (FD) in a CHRIST LPC-16 EPS 2-65DS freeze-dryer, and the vials were stored at $-20^{\circ} \mathrm{C}$.

The Quality Management System of the IRMM Reference Materials Unit is in compliance with the requirements of ISO Guide 34 and ISO 17025. Characterisation of RMs is performed according to the requirements given by ISO Guide 35.

\section{Commutability study}

Commutability as a property of genetic RMs can be described as showing behaviour similar to usual clinical samples giving the true result when investigated by different routine genotyping procedures. To evaluate the commutability of the RMs, a field study was performed. In the absence of any reference method for genetic testing, RMs have to be evaluated by a consensus of recognised reference laboratories.
Table 1 Concentrations measured by the PicoGreen method and copy numbers of the stocks of plasmids pIRMM-0001 (wild-type) and pIRMM-0002 (containing the G20210A mutation).

\begin{tabular}{lll}
\hline Plasmid & $\begin{array}{l}\text { DNA } \\
\text { concentration, } \\
\mu \mathrm{g} / \mathrm{mL}\end{array}$ & $\begin{array}{l}\text { Copy number, } \\
\mathrm{cp} / \mathrm{mL}\end{array}$ \\
\hline pIRMM-0001 & 306 & $8.48 \times 10^{13}$ \\
plRMM-0002 & 298 & $8.25 \times 10^{13}$ \\
\hline
\end{tabular}

Copy numbers were calculated from the concentration values on the basis of the size of the plasmids (3310 bp for both).

A total of 16 leading laboratories in the field of testing for genetic mutations in coagulation factor genes were invited to participate in the field study. Of these expert laboratories, 13 responded positively and participated in the study.

Two vials of each genotype and two vials without DNA, but containing the RM matrix, giving a total of eight vials, were coded, graded randomly, named as Samples A-H, and sent on ice to each laboratory for testing.

The testing laboratories were provided with the study protocol, the terms to be used for reporting, and the result reporting form, but had no information on the expected results. In the study protocol, the aim of the study, properties of samples (content and minimal sample intake, stability and storage, warning to users), preparation (reconstitution) of the materials and reporting requirements were described in detail. The following details were required on the result reporting form: title and address of the laboratory; number of genetic tests performed yearly (factor II G20210A and in general); involvement in proficiency testing programmes for Factor II G20210A mutation and the relevant organisation; dates of arrival and testing; storage conditions; and incidental observations on the samples. The laboratories were asked to describe the methods used in detail and to report the genotyping results in a standardised form. Further comments on results were welcomed.

\section{Results}

Analysis of the different colonies revealed that all clones contained the plasmids. DNA concentrations of the plasmid preparations were evaluated by PicoGreen quantitation. The results and calculated copy numbers are listed in Table 1.

\section{Sequencing}

Sequence analysis of the selected fragments showed that the isolated 609-bp sequences were identical to the GenBank reference sequence (accession number M17262, nt 26302-26910), except for the G20210A mutation (corresponding to nt 26784 in the reference sequence) and an $A \rightarrow G$ point mutation at position 20054 (nt 26628 in the GenBank reference sequence). Interestingly, this second mutation was already present in the patient sample and was linked to the mutated sequence. The cloned wild-type fragment corresponded to $100 \%$ of the reported prothrombin sequence. A map of the pUC18 plasmids produced is shown in Figure 1. 


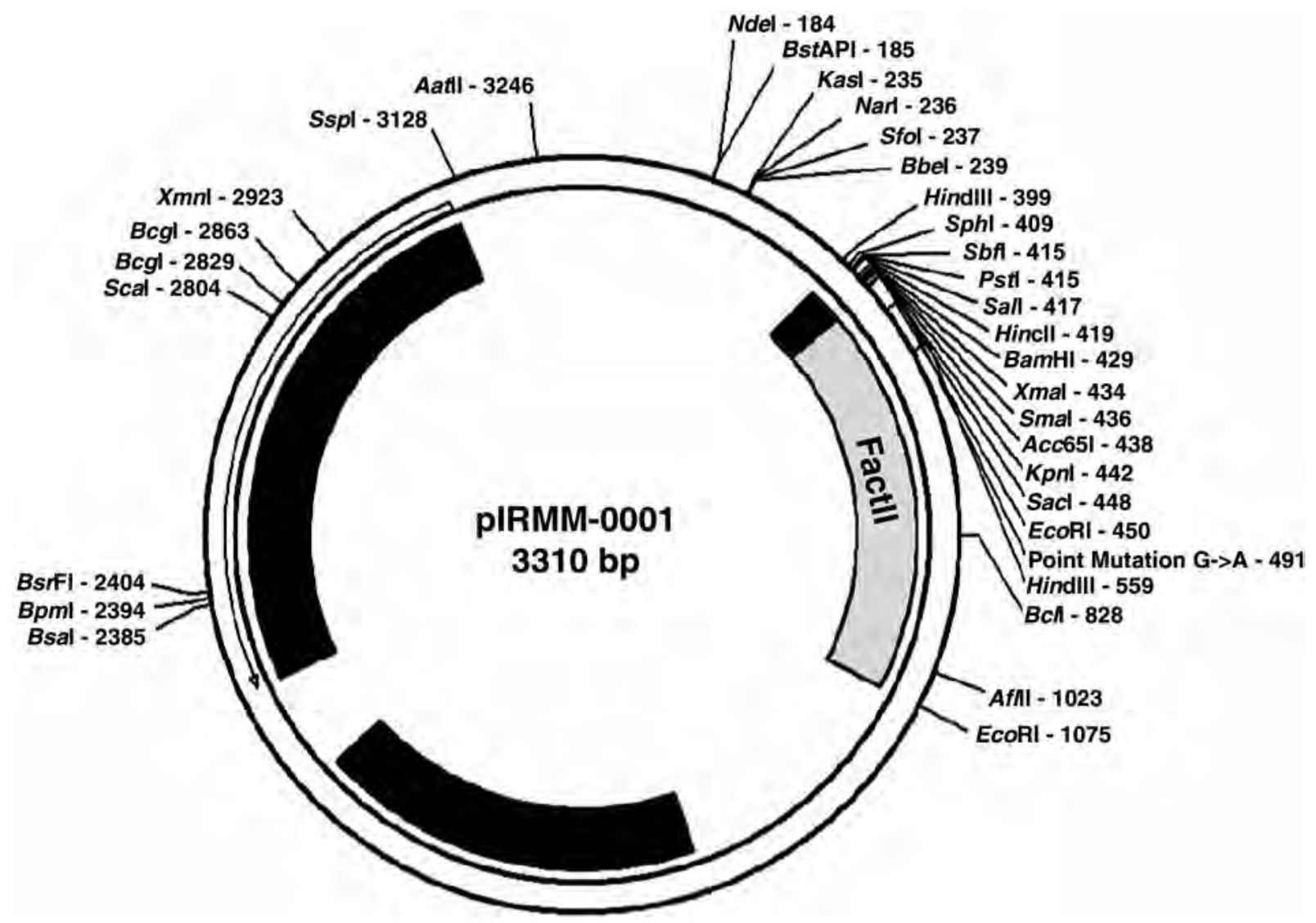

Figure 1 Map of plasmid pIRMM-0001. pIRMM-0001 and pIRMM-0002 are pUC18 plasmids containing the EcoRI fragment of the factor II gene insert from plasmid pCR2.1. The factor II mutation G20210A is located at position 491. Plasmid pIRMM-0001 contains $\mathrm{G}$ at the position corresponding to the wild-type sequence; pIRMM-0002 reads $A$ at the same locus, representing the G20210A mutation.

\section{Commutability study}

For analysis of the samples, nine different methods were used; two of the laboratories applied two different methods, which were reported separately. The method used most often was real-time PCR using the LightCycler (LC) system and kit commercially available from Roche (Roche Diagnostics Belgium, Vilvoorde, Belgium). This method is based on the fluorescence resonance energy transfer (FRET) tech-

Table 2 Methods used in the testing laboratories for determination.

\begin{tabular}{|c|c|c|c|}
\hline Laboratory code & Method(s) & Reference(s) and remark(s) & Primers \\
\hline 1 & LightCycler, Roche kit & Confidential, not communicated & \\
\hline 2 & LightCycler, Roche kit & 32 cycles instead of the original 45 & \\
\hline 3 & PCR-RFLP (HindIII) & $\begin{array}{l}\text { Poort SR et al. (9), modified using an } \\
\text { elongated mutagenic primer }\end{array}$ & A-fw, A-rev \\
\hline 4 & $\begin{array}{l}\text { LightCycler, Roche kit; } \\
\text { PCR-RFLP (HindIII) }\end{array}$ & $\begin{array}{l}\text { Confidential, not communicated } \\
\text { Poort et al. (9) }\end{array}$ & B-fw, B-rev \\
\hline 5 & PCR-RFLP (HindIII) & Danneberg et al. (13) & C-fw, C-rev \\
\hline 6 & PCR-RFLP (HindIII) & In-house method, not published earlier & D-fw, D-rev \\
\hline 7 & $\begin{array}{l}\text { LightCycler, see reference; } \\
\text { PCR-RFLP (HindIII) }\end{array}$ & $\begin{array}{l}\text { Aslanidis et al. (14) } \\
\text { Hybridisation probes: E-det and E-anch } \\
\text { Poort et al. (9) }\end{array}$ & $\begin{array}{l}\text { E-fw, E-rev; } \\
\text { B-fw, B-rev }\end{array}$ \\
\hline 8 & LightCycler, Roche kit & Confidential, not communicated & \\
\hline 9 & Taq-Cycle-Sequencing & $\begin{array}{l}\text { ABI PRISM } 310 \text { Genetic Analyser and } \\
\text { Big Dye Terminator Kit (Applied } \\
\text { Biosystems, Darmstadt, Germany) }\end{array}$ & F-fw, F-rev \\
\hline 10 & PCR-RFLP (HindIII) & Poort et al. (9) & B-fw, B-rev \\
\hline 11 & Mutagenically separated PCR & Endler et al. (15) & $\begin{array}{l}\text { G-fw, G-revA } \\
\text { G-revG }\end{array}$ \\
\hline 12 & LightCycler, Roche kit & Confidential, not communicated & \\
\hline 13 & Mutagenically separated PCR & $\begin{array}{l}\text { In-house method developed with } \\
\text { an industrial partner, confidential }\end{array}$ & \\
\hline
\end{tabular}

Primer and probe sequences known are described in Figure 2. 


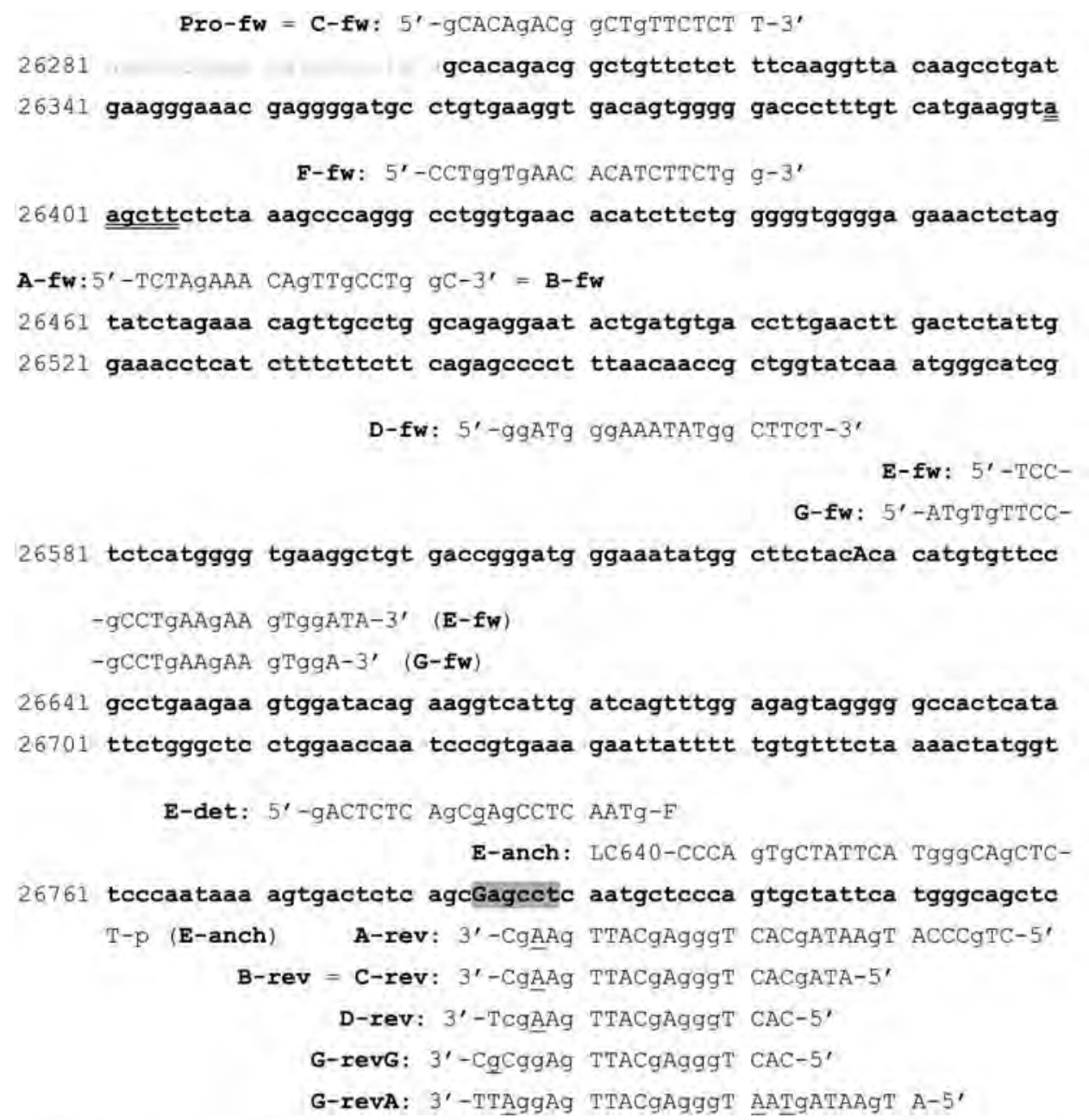

26821 tctgggctca ggaagagcca gtaatactac tggataaaga agacttaaga atccaccacc F-rev: $3^{\prime}$-ATTCT TAggTggTgg-

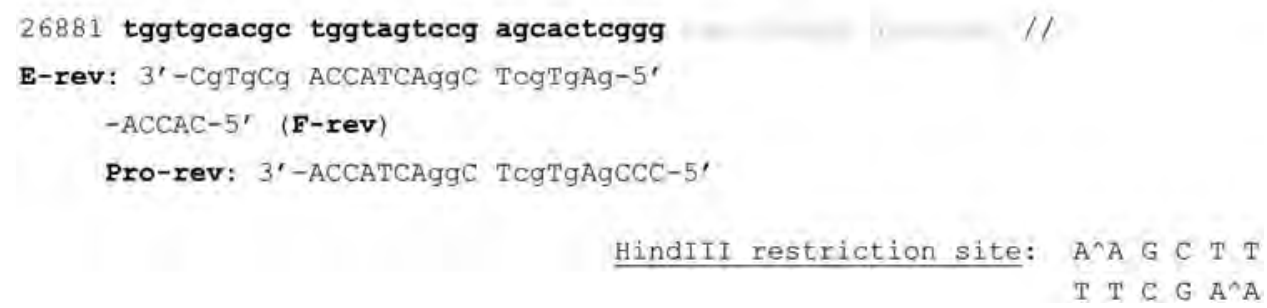

Figure 2 Reference sequence of the prothrombin gene fragment chosen (nt 26302-26910, GenBank accession number M17262) with the positions of primers and probes used by different testing laboratories. The sequence of the wild-type gene fragment inserted fully corresponds to the reference sequence represented by bold characters with position numbers at the beginning of the lines. The G20210A mutation (position nt 26784) is indicated by a capital G. The A $\rightarrow$ G variation at nt 26628 found in the mutated fragment has not been described previously. The position of this mutation is marked by a capital A. It is linked to the G20210A mutation in the selected DNA. Primers and probes used by the testing laboratories are positioned at their annealing sites and marked with the abbreviations used in Table 2. Mismatching nucleotides of PCR primers are underlined. PCR-RFLP methods published use Hindlll digestion for mutation detection following the introduction of a restriction site in the presence of mutation by mutagenic primers. This potential restriction site is highlighted in grey. The prothrombin gene contains another Hindlll restriction site close to the G20210A mutation, which can be used as an internal control for Hindlll digestion (13). This site is double-underlined.

nology. One laboratory used the LightCycler with an in-house FRET method. Six laboratories used PCR amplification followed by Hindlll digestion, and two laboratories used multiplex PCR with allele-specific primers.

Every RM was genotyped correctly and no amplification or genotype was reported for the blank sam- ples. However, one laboratory that used the Roche commercial kit for the LightCycler with a reduced number of cycles (32 instead of 45 recommended in the kit) reported a low signal intensity. When the test was repeated using 45 cycles, the intensity was appropriate. For the mixed RM, to mimic the heterozygous genotype, it was reported that signal intensi- 
ties for both alleles were similar for the different methods.

The testing methods are listed in Table 2. The primers and probes with known sequences used by the testing laboratories are listed in Figure 2.

\section{Discussion}

Suitable CRMs are urgently needed in the field of human genetic testing $(16,17)$.

The advantages of plasmid-type RMs include easy and economical production in large quantities, high reproducibility, versatility, and applicability for qualitative analysis. The major drawback of these genetic RMs is that they cannot be used to assess the quality of DNA extraction procedures. In addition, the length of the fragments inserted is limited. Cloned plasmidtype RMs contain only one defined specific sequence, and similar sequences, which might be of importance when using a certain method, would not be within the scope of the investigation. This restricted commutability represents one limit for the wider use of such RMs.

Assays for defined point mutations, such as the G20210A mutation in the prothrombin gene, usually amplify short fragments, and thus the problem can be tackled by appropriate selection of the fragment inserted into the plasmid.

Most genetic tests for the detection of mutations or polymorphisms are qualitative rather than quantitative - their purpose is to determine the presence or absence of a particular DNA sequence. Accordingly, the concentration of the target sequence is not a key issue, but quantification was the basis for checking for appropriate copy numbers in the RMs. Considering the uncertainty of this calculation and possible DNA degradation during the freeze-drying and reconstitution process, the concentration $\left(5 \times 10^{4}\right.$ copies/vial) given is only a nominal value, supplied as additional material information.

It was demonstrated in the study that the concentration of such plasmid DNA is not critical, as the detection limit of the testing methods is high and low concentrations of test material can usually be compensated by an increase in cycle numbers. Even under assay conditions optimised for high DNA concentrations of test material, when the RMs did not give satisfactory signal intensity, the sample volume could be increased at the expense of water in the PCR test to correct for weak signals. Consequently, in future production of CRMs, the copy numbers could be increased to allow sample sizes of $1 \mu \mathrm{L}$.

From another point of view, such RMs could be a possible source of contamination in PCR analysis if not handled appropriately. Obviously, RMs presented as PCR products or plasmids are good templates for most of the amplification primers utilised and may require specific handling procedures. Appropriate preservation of the RM to keep the copy number of the target sequence low represents an efficient tool to reduce contamination risk.
Sequence analysis confirmed that the reference sequences of the RMs produced are identical to the GenBank reference sequence, except for the G20210A mutation and an $A \rightarrow G$ point mutation at position 20054 in the mutated sequence. This second mutation was linked to the mutated sequence and was already present in the patient sample, which means that it was not introduced during production. To the best of our knowledge, this sequence change has not been reported previously and any associations with pathological conditions are unknown. Recently, an $A \rightarrow G$ exchange at position 19911 has been identified. This mutation seems to affect the splicing efficiency of prothrombin (18). The A20054G mutation did not cause any problems during production of the plasmid-type RMs or in the field study. However, difficulties may arise when a primer is used that binds with its $3^{\prime}$ end to this sequence. Therefore, this sequence alteration is reported in the documentation of the CRMs.

Comparable studies are being performed within the framework of a shared cost project, which is partly funded by the European Commission under FP5 "CRMGEN", in which IRMM is a partner. This is a feasibility study for the development of different RM prototypes in the field of genetic testing, such as plasmid-type RMs.

\section{Conclusion}

In conclusion, in the present study initiated by the IFCC C-MBT, it could be shown that plasmid-type RMs containing defined point mutations can be produced and successfully used in tests for detection of point mutations in a typical QA scheme. At present, the corresponding CRMs have been produced as frozen material, which will be delivered under defined conditions at $-20^{\circ} \mathrm{C}$ until a successful long-term stability study has been carried out to determine the success of freeze-drying techniques. Whether or not the procedure is also suitable for RMs (including CRMs) containing repetitive sequences of variable length still needs to be evaluated in depth.

\section{Acknowledgements}

The project was supported by a grant from the IFCC and complementary finance from the European Commission JRC Institute for Reference Materials and Measurements. A. Torday was supported by Hungarian Scientific Research Fund (OTKA) T034830 and by National Research and Development Programmes (NKFP) 1/024/2001.

\section{References}

1. Dequeker E, Ramsden S, Grody WW, Stenzel TT, Barton DE. Quality control in molecular genetic testing. Nat Rev Genet 2001;2:717-23. 
2. Dequeker E, Cassiman JJ. Evaluation of CFTR gene mutation testing methods in 136 diagnostic laboratories: report of a large European external quality assessment. Eur J Hum Genet 1998;6:165-75.

3. Dequeker E, Cassiman JJ. Genetic testing and quality control in diagnostic laboratories. Nat Genet 2000; 25:259-60.

4. Losekoot M, Bakker B, Laccone F, Stenhouse S, Elles R. A European pilot quality assessment scheme for molecular diagnosis of Huntington's disease. Eur J Hum Genet 1999;7:217-22.

5. Tripodi A, Peyvandi F, Chantarangkul V, Menegatti M, Mannucci PM. Relatively poor performance of clinical laboratories for DNA analyses in the detection of two thrombophilic mutations - a cause for concern. Thromb Haemost 2002;88:690-1.

6. Neumaier M, Braun A, Gessner R, Funke H. Experiences with external quality assessment (EQA) in molecular diagnostics in clinical laboratories in Germany. Working Group of the German Societies for Clinical Chemistry (DGKC) and Laboratory Medicine. Clin Chem Lab Med 2000;38:161-3.

7. Müller MM. Implementation of Reference Systems in Laboratory Medicine. Clin Chem 2000;46:1907-9.

8. EU Lex. Directive 98/79/EC of the European Parliament and of the Council on in vitro Diagnostic Medical Devices of 27 October 1998. Off J Eur Comm 1998;L331:1-37.

9. Poort SR, Rosendaal FR, Reitsma PH, Bertina RM. A common genetic variation in the 39-untranslated region of the prothrombin gene is associated with elevated plasma prothrombin levels and an increase in venous thrombosis. Blood 1996;88:3698-703.

10. Endler G, Mannhalter C. Polymorphisms in coagulation factor genes and their impact on arterial and venous thrombosis. Clin Chim Acta 2003;330:31-55.
11. McGlennen RC, Key NS. Clinical and Laboratory Management of the Prothrombin G20210A Mutation. Arch Pathol Lab Med 2002;126:1319-25.

12. Pauwels J, Schimmel H, Lamberty A. Criteria for the certification of internationally acceptable reference materials. Clin Biochem 1998;31:437-9.

13. Danneberg J, Abbes AP, Bruggeman BJ, Engel H, Gerrits $\mathrm{J}$, Martens A. Reliable genotyping of the G-20210-A mutation of coagulation factor II (prothrombin). Clin Chem 1998;44:349-51.

14. Aslanidis C, Nauck M, Schmitz G. High-speed prothrombin $\mathrm{G} \rightarrow \mathrm{A} 20210$ and methylenetetrahydrofolate reductase $\mathrm{C} \rightarrow \mathrm{T} 677$ mutation detection using real-time fluorescence PCR and melting curves. Biotechniques 1999;27:234-8.

15. Endler G, Kyrle PA, Eichinger S, Exner M, Mannhalter C. Multiplexed mutagenically separated PCR: simultaneous single-tube detection of the factor $V$ R506Q (G1691A), the prothrombin G20210A, and the methylenetetrahydrofolate reductase A223V (C677T) variants. Clin Chem 2001; 47:333-5

16. Ibarreta D, Elles R, Cassiman JJ, Rodriguez-Cerezo E, Dequeker E. Towards quality assurance and harmonization of genetic testing services in the European Union. Nat Biotechnol 2004;10:1230-5.

17. Barton D, Klein CL, Stacey G. Certified reference materials for genetic testing. In: Fuchs J, Podda M, editors. Encyclopedia of diagnostic genomics and proteomics. New York: Marcel Dekker, 2005:226-31.

18. von Ahsen N, Oellerich $\mathrm{M}$. The intronic prothrombin $19911 A \rightarrow G$ polymorphism influences splicing efficiency and modulates effects of the $20210 \mathrm{G} \rightarrow$ A polymorphism on mRNA amount and expression in a stable reporter gene assay system. Blood 2004;103:586-93.

Received March 7, 2005, accepted June 17, 2005 
II. 
Certification of a Reference Material consisting of purified plasmid DNA containing a fragment from the human prothrombin gene (wildtype)

\section{Certified Reference Material IRMM/IFCC-490}

D. Gancberg, J. Marki-Zay, P. Corbisier, C. Klein, H. Schimmel, H. Emons

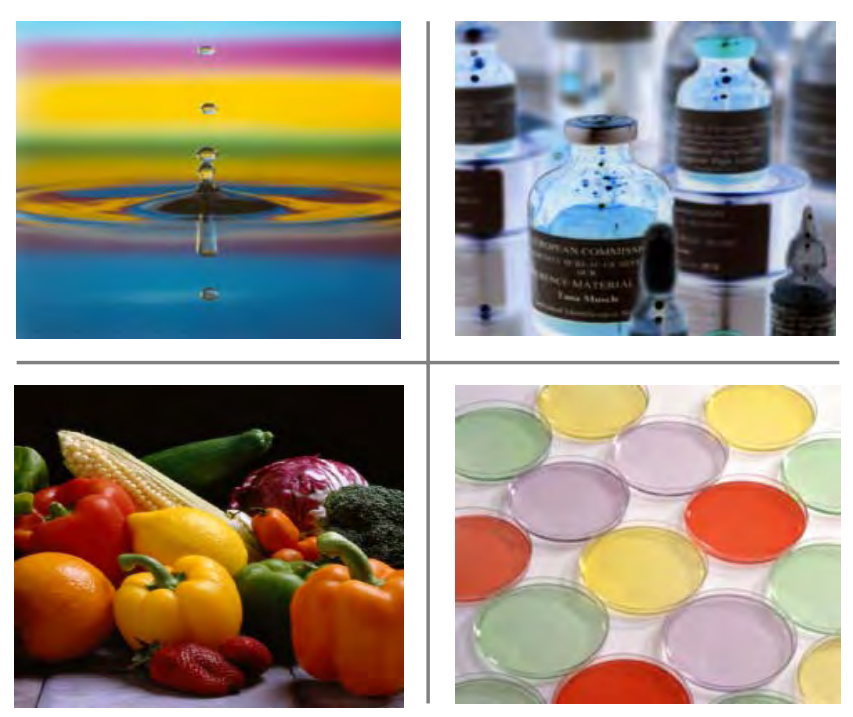


The mission of IRMM is to promote a common and reliable European measurement system in support of EU policies.

\section{European Commission}

Directorate-General Joint Research Centre

Institute for Reference Materials and Measurements

\section{Contact information}

\section{Gancberg}

European Commission

Directorate-General Joint Research Centre

Institute for Reference Materials and Measurements

Retieseweg 111

B-2440 Geel • Belgium

E-mail: david.gancberg@ec.europa.eu

Tel.: +32 (0)14 571911

Fax: +32 (0)14571 548

http://www.irmm.jrc.be

http://www.jrc.cec.eu.int

\section{Legal Notice}

Neither the European Commission nor any person acting on behalf of the Commission is responsible for the use which might be made of the following information.

A great deal of additional information on the European Union is available on the Internet. It can be accessed through the Europa server

http://europa.eu.int

\section{EUR Report 22169 EN}

Luxembourg: Office for Official Publications of the European Communities

ISBN 92-79-01633-4

ISSN 1018-5593

(c) European Communities, 2006

Reproduction is authorised provided the source is acknowledged 
IRMM information

REFERENCE MATERIALS

\title{
Certification of a Reference Material consisting of purified plasmid DNA containing a fragment from the human prothrombin gene (wildtype)
}

\section{IRMM/IFCC-490}

D. Gancberg, J. Marki-Zay, P. Corbusier, H. Schimmel, H. Emons

European Commission

Joint Research Centre

Institute for Reference Materials and Measurements (IRMM)

Retieseweg 111, 2440 Geel, Belgium

\author{
C.Klein \\ Directorate General Joint Research Centre \\ Institute for Health and Consumer Protection \\ Via Fermi, 1 \\ IT-21020 Ispra (Varese), Italy
}

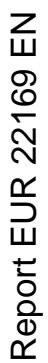

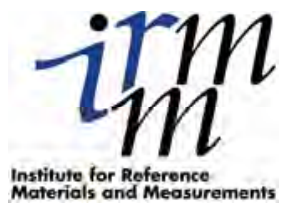




\section{SUMMARY}

This report describes the production and certification of a reference material consisting of purified plasmid DNA (pDNA) pUC18 containing a 609 base pairs fragment of human Factor II (prothrombin) gene sequence, IRMM/IFCC-490. This certified reference material (CRM) is intended to support the validation and the harmonisation of polymerase chain reaction (PCR)-based methods used for detection of the G20210A mutation in the human prothrombin gene. The homogeneity and short-term stability of the batch was assessed by monitoring the melting temperature $\left(T_{m}\right)$ of the product and the crossing point threshold $(C p)$ value obtained after PCR amplification using the LightCycler Factor II (prothrombin) G20210A kit and a LightCycler 1.5 instrument. The long-term stability was assessed using the cycle threshold value $(\mathrm{Ct})$ obtained after real-time PCR amplification using specific primers and probe in combination with $A B I$ Prism instruments.

The material is homogeneous and stable at $-20^{\circ} \mathrm{C}$. The sequence of the insert was confirmed by DNA sequence analysis. Each vial contains an indicative volume of 50 $\mu \mathrm{L}$ corresponding to approximately $1 \mathrm{ng}$ of pDNA. 


\section{TABLE OF CONTENTS}

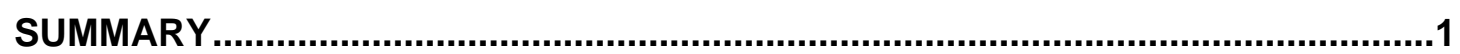

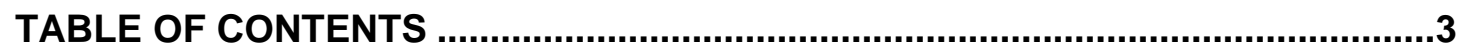

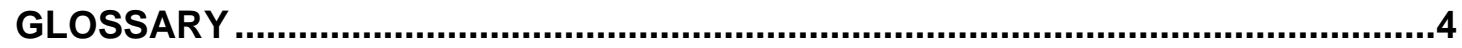

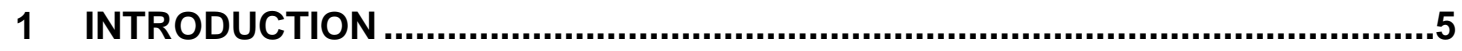

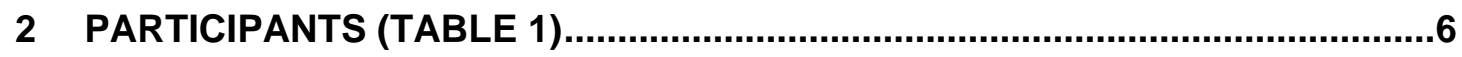

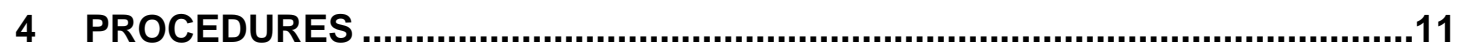

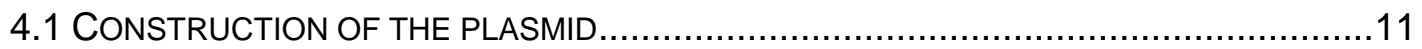

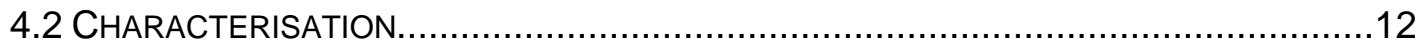

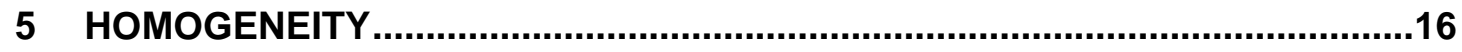

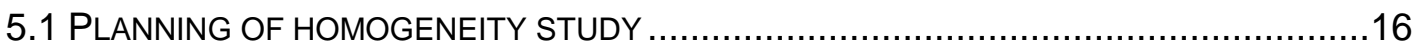

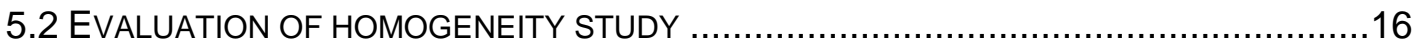

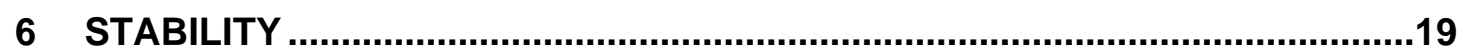

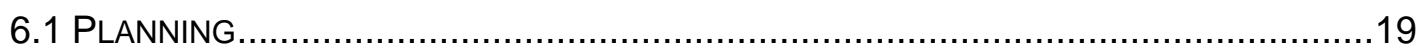

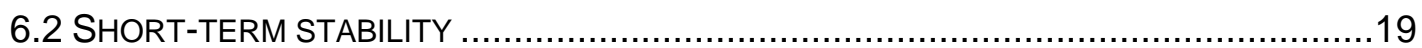

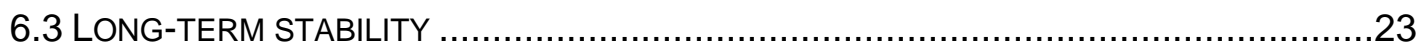

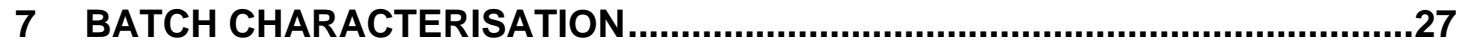

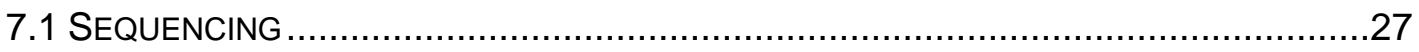

8 VALIDATION

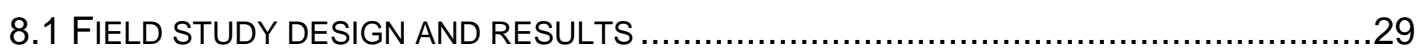

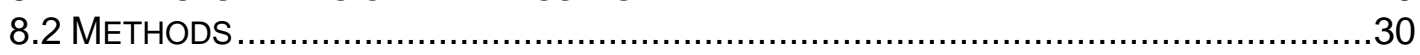

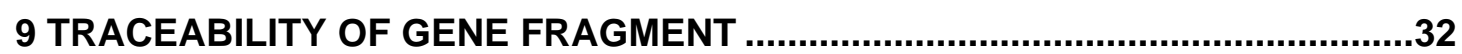

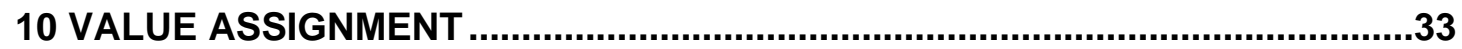

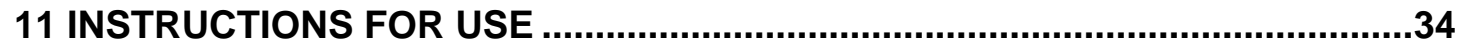

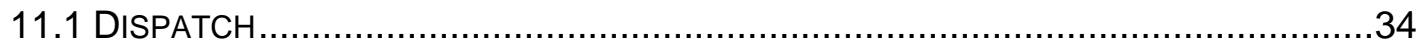

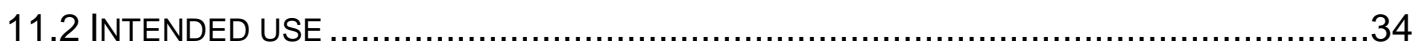

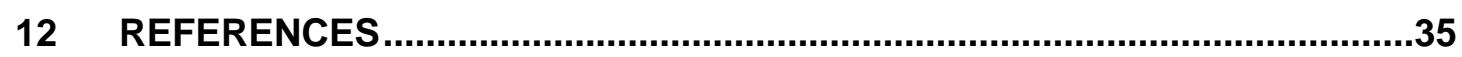




\section{GLOSSARY}

A
ANOVA
b
bp
C
Cp

CRM

$\mathrm{Ct}$

\section{DNA \\ DTCS \\ dNTP \\ FAM \\ FRET \\ $g$ \\ G}

G20210A

IFCC

IP

IRMM

LB

$M S_{\text {among }}$

$M S_{\text {within }}$

$N$

$p$

PCR

pDNA

RFLP

rpm

$s_{b b}$

$S$

SOC

SS

$t_{\alpha, v}$

$t$

$\mathrm{T}$

TAMRA

TBE

TE

$\mathrm{T}_{\mathrm{m}}$

$u_{\mathrm{b}}{ }^{*}$

Adenine

analysis of variance

slope of the regression line

base pairs

Cytosine

Crossing point. The $\mathrm{Cp}$ value is defined as the crossing point

threshold and defines the threshold above which an amplification signal can be detected using real-time PCR.

Certified Reference Material

Cycle threshold. The Ct value is defined as the cycle threshold and defines the threshold above which an amplification signal can be detected using real-time PCR.

deoxyribo nucleic acid

Dye Terminator Cycle Sequencing

deoxy nucleotide triphosphate

6-carboxy fluorescein

Fluorescence Resonance Energy Transfer

centrifugal force

Guanine

mutation at position 20210 resulting from the replacement of a

Guanine by an Adenine in the sequence

International Federation of Clinical Chemistry and Laboratory

Medicine

Intellectual Property

Institute for Reference Materials and Measurements

Luria Broth

mean of squares among vials (from ANOVA)

mean of squares within vials (from ANOVA)

total number of vials in the homogeneity study

probability

polymerase chain reaction

plasmid deoxyribo nucleic acid

Restriction Fragment Length Polymorphism

revolution per minute

standard deviation between the vials

absolute standard deviation

glucose-enriched bacterial medium

the sum of the squares of the data

t-value (two-sided) for a confidence level $\alpha$ and $v$ degrees of

freedom (from regression analysis)

time

Thymine

6-carboxy-tetra-methyl-rhodamine

$8.9 \mathrm{mmol} / \mathrm{L}$ Tris, $8.9 \mathrm{mmol} / \mathrm{L}$ Boric acid, $0.2 \mathrm{mmol} / \mathrm{L}$ EDTA, $\mathrm{pH} 8.3$

$10 \mathrm{mmol} / \mathrm{L}$ Tris, $1 \mathrm{mmol} / \mathrm{L}$ EDTA, $\mathrm{pH} 8.0$

melting temperature. The melting temperature is the temperature at which $50 \%$ of the oligonucleotides are in double-stranded conformation and $50 \%$ are single stranded.

standard uncertainty of the slope of the regression line standard uncertainty due to inhomogeneity that can be hidden by method repeatability. It is used as the minimum uncertainty contribution from inhomogeneity between vials.

degrees of freedom 


\section{Introduction}

Molecular genetic tests are used in medical diagnostics to identify changes in DNA sequences to diagnose or predict an inherited disorder. These tests should be highly selective. The use of an adequate reference material to validate test equipment and measurement procedures is an important part of any quality control system. Currently, only few CRMs are available for molecular genetic testing.

Thus, in collaboration with the International Federation of Clinical Chemistry and Laboratory Medicine (IFCC), a DNA reference material was produced for the detection of a single point mutation (G20210A) in the gene coding for the human prothrombin (Factor II). This gene mutation has been identified as one of the risk factors in venous thrombosis events.

Currently, several measurement procedures are used among laboratories to detect the G20210A mutation in the prothrombin gene. All of them are based on a PCR amplification followed either by restriction enzyme digestion, hybridisation protocols, single-strand conformation polymorphism analysis, LightCycler PCR analysis, denaturating gradient gel analysis or sequencing.

Therefore, a DNA fragment that spans the area of the human prothrombin gene containing the G20210A mutation has been used to produce the reference material. This specific region can be amplified with all primer combinations described in the literature for prothrombin mutation analysis.

Besides the mutated DNA fragment, the wildtype DNA fragment was produced as well. Both fragments were cloned separately into the pUC18 plasmid. The concentration per vial was adjusted to $1 \mathrm{ng}$ per $50 \mu \mathrm{L}$.

For the present CRM IRMM/IFCC-490, a batch of 1500 vials of purified pDNA containing a cloned $609 \mathrm{bp}$ fragment of the human prothrombin gene was produced. The certification of IRMM/IFCC-490 was performed according to IRMM RM Unit procedures applying the ISO Guide 34 quality management system [1-4]. Stability measurements were performed at different temperatures $\left(-20^{\circ} \mathrm{C}, 4^{\circ} \mathrm{C}, 18^{\circ} \mathrm{C}, 40^{\circ} \mathrm{C}\right)$. Sequence identity in the production batch before dilution was assessed by DNA sequence analysis of the fragment inserted into the pUC18 vector.

The produced batch was characterised for homogeneity.

Samples in short-term stability measurements were analysed by melting curve experiment using PCR and the commercial LightCycler kit for Factor II (prothrombin) G20210A mutation detection [5]. Samples in long-term stability measurements were analysed by real-time PCR experiments using specific primers and probe. 


\section{Participants (Table 1)}

The following laboratories with expertise in genetic testing were involved in the field study:

Table 1: List of participants for the characterisation of the material

\begin{tabular}{|l|l|l|}
\hline Halbmayer, W. & $\begin{array}{l}\text { Institut für Medizinische und Chemische } \\
\text { Labordiagnostik mit Thromboseambulanz I } \\
\text { Krankenhaus der Stadt Wien Lainz }\end{array}$ & AT-1130 Wien \\
\hline Zadro, R. & $\begin{array}{l}\text { Clinical Institute of Laboratory Diagnosis / Clinical } \\
\text { Hospital Center Zagreb University School of } \\
\text { Medicine }\end{array}$ & HR-10000 Zagreb \\
\hline Melegh, B. & $\begin{array}{l}\text { University of Pecs, Department of Medical } \\
\text { Genetics and Child Development }\end{array}$ & HU-7624 Pecs \\
\hline Gemmati, D. & $\begin{array}{l}\text { Ctr. Study Haemostasis and Thrombosis, } \\
\text { University of Ferrara }\end{array}$ & IT-44100 Ferrara \\
\hline Spannagl, M. & $\begin{array}{l}\text { Ludwig-Maximilians-Universität, Klinikum } \\
\text { Innenstadt, Hämostaseologische Ambulanz }\end{array}$ & DE-80336 München \\
\hline Kitchen, S. & $\begin{array}{l}\text { Dept. of Coagulation / Sheffield Haemophilia and } \\
\text { Thrombosis Centre / Royal Hallamshire Hospital }\end{array}$ & GB-S102 JF Sheffield \\
\hline Mannhalter, C. & $\begin{array}{l}\text { Klinisches Institut für Medizinische und } \\
\text { Chemische Labordiagnostik (KIMCL) }\end{array}$ & AT-1090 Wien \\
\hline
\end{tabular}




\section{Processing steps}

Prof. C. Mannhalter, Clinical Institute of Medical and Chemical Laboratory Diagnostics, General Hospital and Medical University Vienna (Vienna, Austria) isolated and purified a $609 \mathrm{bp}$ fragment of the human prothrombin gene and cloned it into vector pCR2.1 (TA Cloning ${ }^{\circledR}$ kit, Invitrogen). A solution with approximately $10^{6}$ copies of this plasmid in $100 \mu \mathrm{L}$ TE buffer pH 8.0 was delivered frozen to IRMM. The production and certification of IRMM/IFCC-490 was performed at IRMM.

The processing steps for the production of IRMM/IFCC-490 are described by Klein et al. [6] and in the procedures of Chapter 4. As the pCR2.1 vector is patented, it was necessary to subclone the fragment in a non-IP protected vector. Briefly, the $609 \mathrm{bp}$ fragment was subcloned into the pUC18 cloning vector (Figure 1). Two primers, designed to obtain the Factor II insert of the pCR2.1 plasmid flanked by a EcoRI restriction site, were used to amplify a fragment that could subsequently be inserted into pUC18, via the unique EcoRI site.

Qualitative PCR was applied (see Chapter 4) and $5 \mu \mathrm{L}$ of the plasmid (at $10^{4}$ copies/ $\mu \mathrm{L}$ ) were amplified using primers $\mathrm{P}-0342$ and $\mathrm{P}-0343$ (Table 2).

Table 2: Primers used for cloning in EcoRI site

\begin{tabular}{|l|l|l|}
\hline & $\begin{array}{l}\text { IRMM Primer Code } \\
\text { number }\end{array}$ & Primer sequence \\
\hline Forward & P-0342 & 5'-CGAGCTCGGATCCACTAGTAA-3' \\
\hline Reverse & P-0343 & 5'-GCCAGTGTGATGGATATCTGC-3' \\
\hline
\end{tabular}

The amplification products were controlled by agarose gel electrophoresis for their correct molar mass (length in bp) by comparison to a DNA molecular mass ladder and purified using the Qiagen PCR fragment purification kit. Subsequently, PCR fragments and pUC18 plasmid were digested with EcoRI.

After the digestion, the restriction enzyme was heat-inactivated for $20 \mathrm{~min}$ at $65{ }^{\circ} \mathrm{C}$ and the linearised vector pUC18 was dephosphorylated for 30 min at $37^{\circ} \mathrm{C}$ using the shrimp alkaline phosphatase in order to prevent recircularisation of the plasmid. The dephosphorylated vector and the insert were then ligated overnight at $14{ }^{\circ} \mathrm{C}$. The resulting new plasmid containing the 609 bp wildtype fragment of Factor II was coded pIRMM-0001. 


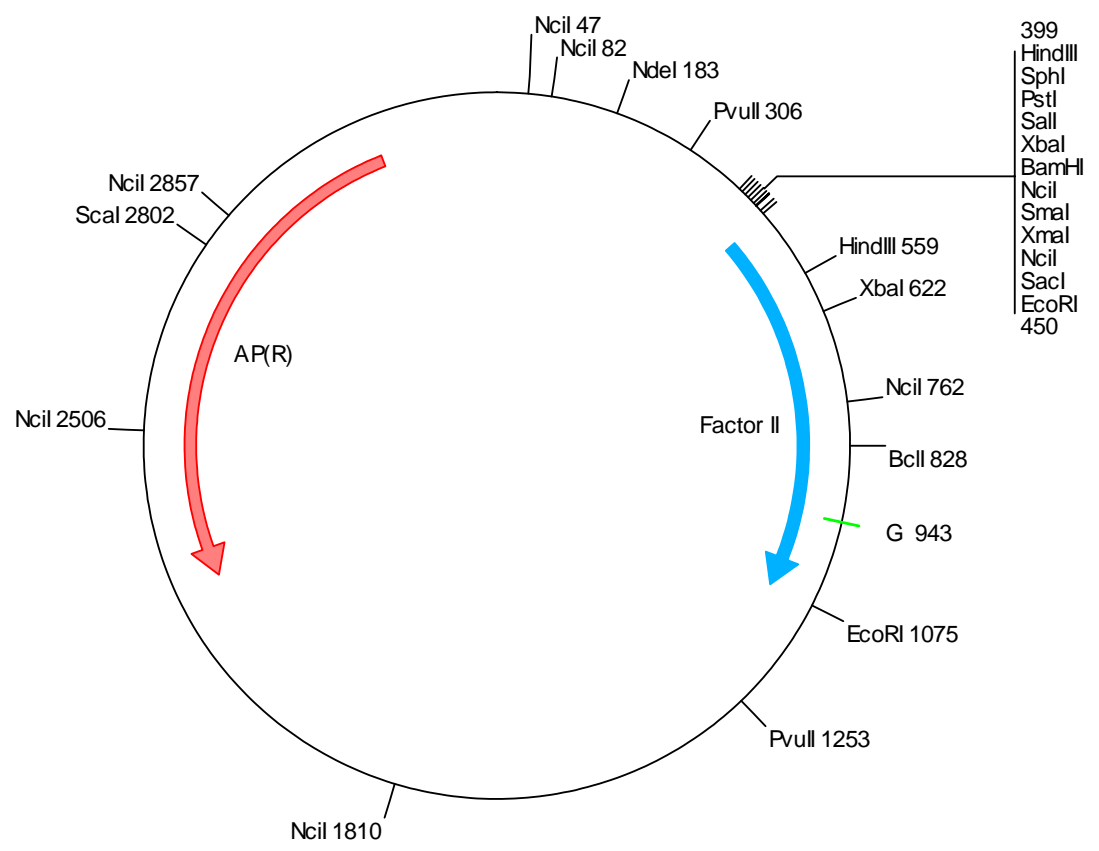

Figure 1: Map of plasmid pIRMM-0001 (3311 bp, wildtype). The Factor II mutation G->A is located at position 943 (in plasmid pIRMM-0002).

The resulting plasmid pIRMM-0001 was transformed into E. coli TOP10.

Ten colonies of bacteria were tested for the presence of the insert. Therefore, $a$ sample of each colony was introduced as template in a qualitative PCR reaction mix using the specific Factor II primers (P-0308 and P-0309, Table 3). The following PCR cycling programme was used in the GeneAmp 9700 System: melting pDNA at $95^{\circ} \mathrm{C}$ for $10 \mathrm{~min} ; 40$ cycles of denaturation at $95^{\circ} \mathrm{C}$ for $1 \mathrm{~min}$, annealing at $58^{\circ} \mathrm{C}$ for $1 \mathrm{~min}$, extension at $72{ }^{\circ} \mathrm{C}$ for $1 \mathrm{~min}$; incubation at $72{ }^{\circ} \mathrm{C}$ for $10 \mathrm{~min}$; hold at $4{ }^{\circ} \mathrm{C}$.

Table 3: Primers used for Factor II sequence amplification

\begin{tabular}{|l|l|l|}
\hline & $\begin{array}{l}\text { IRMM Primer Code } \\
\text { number }\end{array}$ & Primer sequence \\
\hline Forward & P-0308 & 5'-GCACAGACGGCTGTTCTCTT-3' \\
\hline Reverse & P-0309 & 5'-CCCGAGTGCTCGGACTACCA-3' \\
\hline
\end{tabular}

The analysis of the different colonies revealed that all colonies contained the plasmids. Clone "number 4" was selected and a HindIII and EcoRI digest was carried out in order to assess the orientation of the insert (Figure 2). 


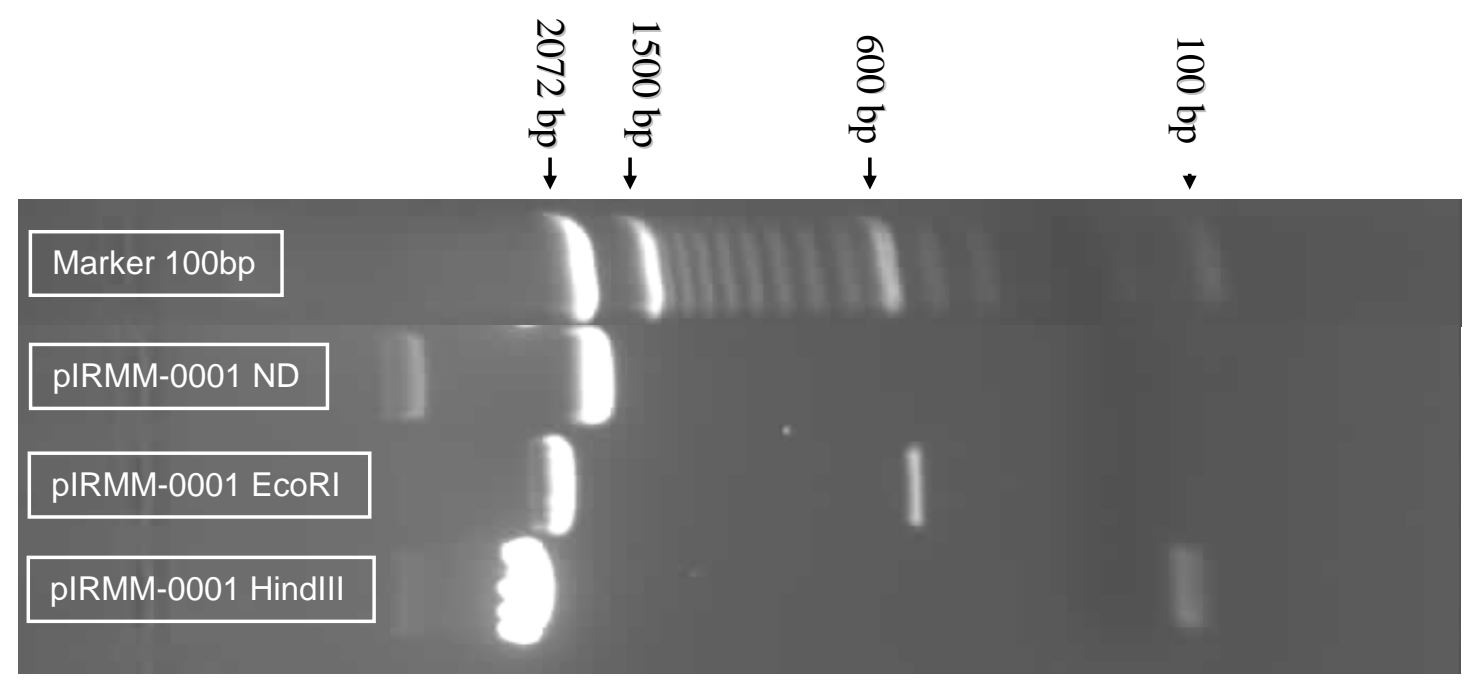

Figure 2. Agarose gel (mass fraction = $1 \%$ ) electrophoresis of pIRMM-0001 $10 \mu \mathrm{L}$ of pDNA digested with EcoRI (lane 3), with HindIII (lane 4), or not digested (ND, lane 2) were mixed with $10 \mu \mathrm{L}$ DNase free water and $2.2 \mu \mathrm{L}$ of 10-times concentrated gel loading buffer. The agarose gel in TBE buffer was stained with 0.5 $\mu \mathrm{g} / \mathrm{mL}$ Ethidium Bromide; the electrophoresis was performed using the following conditions: 100 V, 45 min; marker: 100 bp Ready-Load DNA (Invitrogen, Merelbeke, Belgium, lane 1).

The undigested plasmid exists under the supercoiled (heavier) and linear forms. The EcoRI digestion generated fragment sizes of $625 \mathrm{bp}$ and $2686 \mathrm{bp}$. If the fragment is in the desired orientation (see Figure 1), fragment sizes of $160 \mathrm{bp}$ and $3151 \mathrm{bp}$ should be obtained after Hindlll digestion. The size of the fragments generated after digestion indicated that the insert was in the desired orientation. Two clones containing the sequence of plasmid pIRMM-0001 were kept as glycerol stock at -70 ${ }^{\circ} \mathrm{C}$ and coded C-0001 (clone 4) and C-0003 (clone 1).

The plasmids from strain C-0001 were purified on large scale using the QIAgen plasmid purification Gigakit and the concentration of the plasmids was measured by double-stranded DNA PicoGreen quantitation method [7]. The average pDNA stock concentration and standard deviation was $1390 \pm 29 \mu \mathrm{g} / \mathrm{mL}$.

\section{Production}

The pDNA production follows the QIAgen Gigakit plasmid purification protocol.

1. A bacterial culture ( $10 \mathrm{~mL}$ of $\mathrm{LB}$ medium $+100 \mu \mathrm{g} / \mathrm{mL}$ ampicillin) was inoculated with $100 \mu \mathrm{L}$ of strain C-0001 from glycerol stock containing the pIRMM-0001 plasmid and grown at $37^{\circ} \mathrm{C}$ for $8 \mathrm{~h}$ with orbital shaking at $300 \mathrm{rpm}$. At the end of the day a LB agar plate containing $100 \mu \mathrm{g} / \mathrm{mL}$ ampicillin was plated out and placed at $37^{\circ} \mathrm{C}$ overnight (in order to be able to pick up a single colony the next day).

2. A single colony was cultivated as primary culture at $37^{\circ} \mathrm{C}$ for $8 \mathrm{~h}$ in $10 \mathrm{~mL} \mathrm{LB}$ medium $+100 \mu \mathrm{g} / \mathrm{mL}$ ampicillin with orbital shaking at $300 \mathrm{rpm}$. Four flasks of 500 
$\mathrm{mL}$ LB medium $+100 \mu \mathrm{g} / \mathrm{mL}$ ampicillin were then incubated overnight with $2.5 \mathrm{~mL}$ of a primary culture and grown at $37^{\circ} \mathrm{C}$ with orbital shaking at $275 \mathrm{rpm}$.

3. Bacterial cells were centrifugated for $15 \mathrm{~min}$ at $6000 \mathrm{~g}$ at $4{ }^{\circ} \mathrm{C}$ using a Avanti J-20 Beckman centrifuge. Plasmid DNA was purified according to the procedure from QIAgen plasmid purification (second edition, August 2003 [8]).

4. The plasmid DNA pellet obtained at the end of the purification was resuspended in $5 \mathrm{~mL}$ TE pH 8.0 overnight, aliquoted in $5 \times 1 \mathrm{~mL}$ and stored at $-20^{\circ} \mathrm{C}$.

5. The concentration of double-stranded pDNA was determined by fluorescence of PicoGreen [7] using lambda phage DNA as external standard. The integrity of pDNA was verified visibly by agarose gel electrophoresis.

6. The plasmid stock solution was diluted in $\mathrm{TE} \mathrm{pH} 8.0$ to a final concentration of $0.02 \mathrm{ng} \mathrm{pDNA} / \mu \mathrm{L}$ or $1 \mathrm{ng} / 50 \mu \mathrm{L}$.

7. One thousand five hundred polypropylene vials (sterile, $1.5 \mathrm{~mL}$ volume) were filled manually with $50 \mu \mathrm{L}$ of pDNA solution (mass concentration $0.02 \mathrm{ng} / \mu \mathrm{L}$ TE buffer $\mathrm{pH}$ 8.0).

8. The vials were closed with screw caps in a laminar flowhood and labelled.

9. Homogeneity and stability studies as well as batch characterisation were carried out. 


\section{Procedures}

\subsection{Construction of the plasmid}

\section{Enzymatic Digestion}

The digestion reaction was composed of $10 \mu \mathrm{L}$ of plasmid, $3 \mu \mathrm{L}$ buffer 10-times concentrated (SURE Cut buffer H for EcoRI, SURE Cut buffer B for HindIII, Roche, Vilvoorde, Belgium), $16 \mu \mathrm{L}$ Promega nuclease-free water and $1 \mu \mathrm{L}$ enzyme (either HindIII or EcoRI, Promega, Leiden, The Netherlands).

\section{Dephosphorylation}

The reaction mix for dephosphorylation was prepared by mixing $10 \mu \mathrm{L}$ plasmid linearized (by EcoRI), $2 \mu \mathrm{L}$ buffer 10-times concentrated (Roche, Vilvoorde, Belgium), $8 \mu \mathrm{L}$ enzyme shrimp alkaline phosphatase (SAP, Roche,Vilvoorde, Belgium); the SAP was further inactivated at $65^{\circ} \mathrm{C}$ for $15 \mathrm{~min}$.

\section{Ligation}

The reaction mix was prepared as follows: $5 \mu \mathrm{L}$ Promega nuclease-free water, $1 \mu \mathrm{L}$ of 10-times concentrated buffer (Roche, Vilvoorde, Belgium), $1.5 \mu \mathrm{L}$ vector and $1 \mu \mathrm{L}$ insert:, $1 \mu \mathrm{L}$ of T4 DNA ligase (Westburg, Leusden, The Netherlands).

\section{Transformation}

The TOP10 competent E.coli (Invitrogen, Merelbeke, Belgium) were thawed on ice (one shot aliquots) and $2 \mu \mathrm{L}$ of the ligation reaction was added and mixed gently by tapping. After incubation $30 \mathrm{~min}$ on ice, a heat shock was performed at $42{ }^{\circ} \mathrm{C}$ for exactly $30 \mathrm{~s}$. The tube was then placed on ice for $2 \mathrm{~min}$, and $250 \mu \mathrm{L}$ of SOC medium was added to the tube. The tube was then incubated at $37^{\circ} \mathrm{C}$ for exactly $1 \mathrm{~h}$ with shaking at $225 \mathrm{rpm}$. The tube was placed on ice, then $50 \mu \mathrm{L}$ or $200 \mu \mathrm{L}$ of the mixture was plated overnight at $37^{\circ} \mathrm{C}$ on LB Agar medium containing antibiotic $(100 \mu \mathrm{g} / \mathrm{mL}$ ampicillin or kanamycin).

\section{Bacterial media}

LB Broth (Fluka, Bornem, Belgium): dissolve $20 \mathrm{~g} / \mathrm{L}$ (Tryptone $10 \mathrm{~g} / \mathrm{L}$, Yeast extract 5 $\mathrm{g} / \mathrm{L}$, Sodium Chloride $5 \mathrm{~g} / \mathrm{L}$ ) in water, adjust to $\mathrm{pH} 7.2$, sterilize for $15 \mathrm{~min}$ at $121^{\circ} \mathrm{C}$. LB Agar (Fluka, Bornem, Belgium): dissolve $30 \mathrm{~g} / \mathrm{L}$ (Tryptone $10 \mathrm{~g} / \mathrm{L}$, Yeast extract 5 $\mathrm{g} / \mathrm{L}$, Sodium Chloride $5 \mathrm{~g} / \mathrm{L}$, Agar $10 \mathrm{~g} / \mathrm{L}$ ) in water, adjust to $\mathrm{pH} 7.2$, sterilize for 15 $\min$ at $121^{\circ} \mathrm{C}$.

SOC medium (Fluka, Bornem, Belgium): ready-to-use medium, sterile. Contains Tryptone $20 \mathrm{~g} / \mathrm{L}$, Yeast extract $5 \mathrm{~g} / \mathrm{L}$, glucose $20 \mathrm{mmol} / \mathrm{L}$. 


\section{Ampicillin}

$100 \mathrm{mg} / \mathrm{mL}$ Ampicillin sodium salt (Sigma, Belgium, cat\#A0166-5G) was dissolved in water and sterilized by $0.22 \mu \mathrm{m}$ filtering. This solution stock was aliquoted and diluted 1000 -times ( $\mathrm{vol} / \mathrm{vol})$ to reach the working concentration of $100 \mu \mathrm{g} / \mathrm{mL}$.

\section{Agarose gel electrophoresis}

To $10.0 \mu \mathrm{L}$ sample, of either pDNA or PCR product, $2.2 \mu \mathrm{L}$ of 10 -times concentrated gel loading buffer (Invitrogen, Merelbeke, Belgium) was mixed as well as $10 \mu \mathrm{L}$ of Promega nuclease-free water. $20 \mu \mathrm{L}$ of this sample was loaded on a $1 \%$ (mass fraction) agarose gel in TBE buffer with $0.5 \mu \mathrm{g} / \mathrm{mL}$ Ethidium Bromide (Sigma, St. Louis, USA). Electrophoresis was performed at $100 \mathrm{~V}$ for 45 min using a Mini-Sub Cell GT Electrophoresis system and a Power Pac 3000 (Bio-Rad, Nazareth, Belgium). Gels were photographed on a GeneScan Gel Documentation System (Syngene, Cambridge, United Kingdom).

\subsection{Characterisation}

\section{Minimum sample volume}

The minimum sample volume was $5 \mu \mathrm{L}$ as recommended in the protocol of the Factor II (prothrombin) G20210A kit used in combination with the LightCycler. Dilution experiments during the short-term stability study gave an indication of the limit of detection.

\section{Quantification of pDNA}

Quantification of double-stranded pDNA in stock solution was performed with PicoGreen reagent in a microtiter plate using fluorimetry (excitation at $480 \mathrm{~nm}$ and emission at $525 \mathrm{~nm}$ ) [7]. A standard DNA calibration curve was prepared with lambda phage DNA at mass concentrations ranging from 0 to $1 \mathrm{ng} / \mu \mathrm{L}$. The test was prepared in a microtiter plate, and fluorescence was detected in a FLUOstar Galaxy plate reader (BMG LABTECH GmbH, Offenburg, Germany) [9].

\section{Qualitative PCR}

Each reaction mixture for PCR reactions (50 $\mu \mathrm{L}$ total volume) contained PCR buffer, $1.5 \mathrm{mmol} / \mathrm{L} \quad \mathrm{MgCl}_{2}, 200 \mu \mathrm{mol} / \mathrm{L} \quad \mathrm{dNTP}, \quad 0.1 \mu \mathrm{mol} / \mathrm{L}$ forward primer, $0.1 \mu \mathrm{mol} / \mathrm{L}$ reverse primer, 0.5 unit Platinum ${ }^{\circledR}$ Taq DNA polymerase and $5 \mu \mathrm{L}$ pDNA template. PCR primers (Applied Biosystems, Lennik, Belgium) used are summarized in Tables 2 and 3. All other reaction components for PCR reactions were provided by Invitrogen (Merelbeke, Belgium). PCR reactions were performed using the following cycling programme: melting pDNA at $95{ }^{\circ} \mathrm{C}$ for $10 \mathrm{~min}$; 40 cycles of denaturation 95 
${ }^{\circ} \mathrm{C}$ for $1 \mathrm{~min}$, annealing $58^{\circ} \mathrm{C}$ for $1 \mathrm{~min}$, extension $72{ }^{\circ} \mathrm{C}$ for $1 \mathrm{~min}$; incubation at 72 ${ }^{\circ} \mathrm{C}$ for $10 \mathrm{~min}$; hold at $4{ }^{\circ} \mathrm{C}$. PCR products were visualised by agarose gel electrophoresis.

\section{Quantitative real-time PCR}

The principle of the quantitative real-time PCR is the use of a specific fluorescent probe in addition to the primers. The fluorescence intensity is proportional to the number of target DNA sequences. This fluorescence is recorded on-line during the amplification process. The TaqMan ${ }^{\circledR}$ probe contains a reporter dye (FAM) at its 5' end and a quencher (TAMRA) dye at its 3' end. Accumulation of PCR products is detected directly by monitoring the increase in fluorescence of the reporter dye. The number of cycles which are required to pass a fluorescence threshold (Ct-value) correlates with the amount of target DNA in the original sample.

We used the ABI Prism 7700 and ABI Prism 7900HT device (Applied Biosystems, Lennik, Belgium).

Each reaction mixture for real-time PCR reactions (25 $\mu \mathrm{L}$ total volume) contained $12.5 \mu \mathrm{L}$ of 2-times concentrated Universal PCR Master Mix (Applied Biosystems, Lennik, Belgium), $2.5 \mu \mathrm{L}$ of forward primer (9 $\mu \mathrm{mol} / \mathrm{L}), 2.5 \mu \mathrm{L}$ of reverse primer $(9$ $\mu \mathrm{mol} / \mathrm{L}), 2.5 \mu \mathrm{L}$ of probe $(2 \mu \mathrm{mol} / \mathrm{L})$ and $5 \mu \mathrm{L}$ pDNA template. The sequences of PCR primers and probe (Applied Biosystems, Lennik, Belgium) used are summarized in Table 4. PCR reactions were performed using the following cycling programme: 2 min at $50{ }^{\circ} \mathrm{C}, 10 \mathrm{~min}$ at $95^{\circ} \mathrm{C},\left(15 \mathrm{~s}\right.$ at $95^{\circ} \mathrm{C}, 1 \mathrm{~min}$ at $\left.60^{\circ} \mathrm{C}\right)$ for 30 cycles. Ct values were obtained using the SDS 2.2.2 software on ABI Prism 7900 HT and SDS 1.9.1 on ABI Prism 7700 instrument.

Each sample was analysed in triplicate. Triplicates were randomized on the 96-wells plates, and an internal control (pDNA at $0.02 \mathrm{ng} / \mu \mathrm{L}$ ) was displayed on each plate to allow comparison of data on the different instruments.

Table 4. Primers and probe used for the quantitative PCR

\begin{tabular}{|l|l|l|}
\hline & $\begin{array}{l}\text { IRMM Primer } \\
\text { Code number }\end{array}$ & Primer sequence \\
\hline Forward & P-0564 & 5'-GCTCACCGGCTCCAGATTT -3' \\
\hline Reverse & P-0565 & 5'- TTCTGCGCTCGGCCC-3' \\
\hline Probe & P-0566 & FAM-5'-TCAGCAATAAACCAGCCAGCCGGA-3'-TAMRA \\
\hline
\end{tabular}




\section{Genotyping using the LightCycler technology}

The LightCycler Factor II (prothrombin) G20210A kit (Roche, cat \#036 10195001, [5]) provides primers and hybridisation probes for sequence-specific detection and includes a control template heterozygous for the G20210A mutation for reliable interpretation, as well as a negative control consisting of DNA-free water.

Principle: combining hybridisation probe detection with subsequent melting curve analysis makes applications such as rapid genotyping and mutation analysis possible (example in Figure $3 \mathrm{~A}$ and $3 \mathrm{~B}$ ). Indeed, a single mismatch between the hybridisation probe and its target sequence, caused by a single point mutation, changes the melting temperature of the bound probe. Therefore, the recording of the melting temperature $\left(T_{m}\right)$ in the plot of the negative first derivative of fluorescence intensity versus temperature $(-\mathrm{dF} / \mathrm{dT})$ allows discrimination between the homozygous (wildtype or mutant) as well as the heterozygous genotypes (example in Figure 3B).

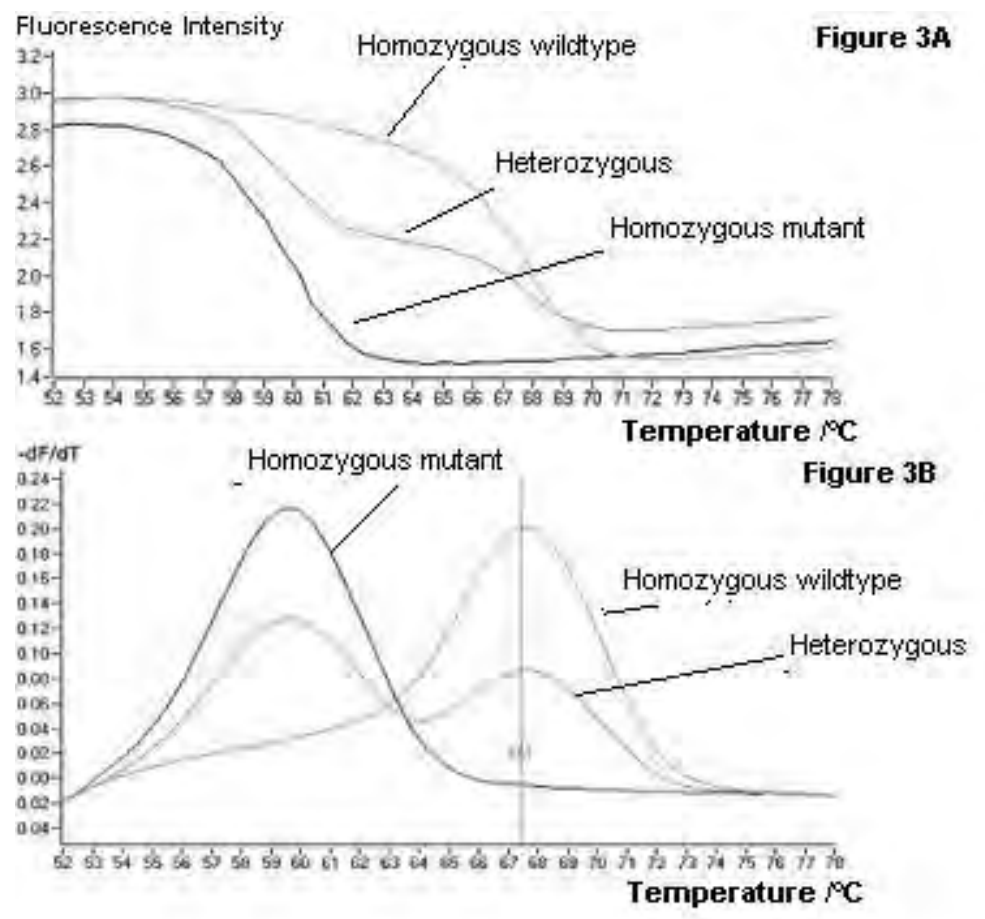

Figure 3: Example of analysis and interpretation of data using the LightCycler technology and the melting curve analysis

The $T_{m}$ does not only depend on the length and $G+C$ content, but also depends on the degree of homology between the mutation probe and the template DNA. In the LightCycler Factor II (prothrombin) G20210A kit, the wildtype genotype and the fluorescein-labeled hybridization probe form a perfect match resulting in a $T_{m}$ of about $59^{\circ} \mathrm{C}$. The kit manual stipulates that the $T_{m}$ values may vary $\pm 2.5^{\circ} \mathrm{C}$ between different experiments. 
Quantitative data on the DNA used as template in the PCR reaction is given by the Crossing point $(\mathrm{Cp})$ threshold obtained "by automatic call" in the program. The Cp value is defined as the crossing point threshold and defines the threshold above which an amplification signal can be detected.

\section{DNA sequencing}

Between 100 and 200 ng of pDNA were used in Dye Terminator Cycle Sequencing (DTCS) reaction, according to the DTCS Chemistry Protocol [10] and using the primers P-0289, P-0419, P-0579 and P-0580 (Table 5). The following thermal cycling programme was used: 30 cycles of $96{ }^{\circ} \mathrm{C}$ for $20 \mathrm{~s}, 50{ }^{\circ} \mathrm{C}$ for $20 \mathrm{~s}, 60^{\circ} \mathrm{C}$ for 4 min and hold at $4{ }^{\circ} \mathrm{C}$. Samples were analysed on a $\mathrm{CEQ}^{\mathrm{TM}} 8000$ Genetic Analysis System (Beckman Coulter, Inc., Fullerton, CA, USA), using the following method: denaturation $120 \mathrm{~s}$ at $90{ }^{\circ} \mathrm{C}$, injection $15 \mathrm{~s}$ at $2.0 \mathrm{kV}$, separation $85.0 \mathrm{~min}$ at $4.2 \mathrm{kV}$.

Table 5: Primers used for Factor II sequencing

\begin{tabular}{|l|l|l|}
\hline & $\begin{array}{l}\text { IRMM Primer } \\
\text { Code number }\end{array}$ & Primer sequence \\
\hline Forward (M13-F) & P-0419 & 5'- GTA AAA CGA CGG CCA G-3' \\
\hline Reverse (M13-R) & P-0289 & 5'- CAG GAA ACA GCT ATG AC-3' \\
\hline Forward (Factor II-F) & P-0579 & 5'- TAC TGA TGT GAC CTT GAA CT-3' \\
\hline Reverse (Factor II-R) & P-0580 & 5'- CTT CTG TAT CCA CTT CTT CA-3' \\
\hline
\end{tabular}




\section{Homogeneity}

\subsection{Planning of homogeneity study}

1500 vials were produced. The minimum required number of vials used in the homogeneity study $(N)$ is calculated as ${ }^{3} \sqrt{1500}=12$ [1-2]. The homogeneity study was performed using data from samples kept at $-70{ }^{\circ} \mathrm{C}(N=6)$ and at $-20^{\circ} \mathrm{C}(\mathrm{N}=18)$ in the short-term stability study. Two replicates of $5 \mu \mathrm{L}$ of IRMM/IFCC-490 were analysed using the LightCycler Factor II (prothrombin) G20210A mutation detection kit and the Cp values were assessed.

The average of the melting temperature of the product was recorded additionally as it confirmed the identity of the sequence (using defined primers and probes).

\subsection{Evaluation of homogeneity study}

The results of the Crossing point threshold (Cp) and average of the $T_{m}$ measurements are summarized in Table 6 . The positive control was a fragment of human DNA, heterozygous for the mutation, and the negative control was DNA-free water (both are included in the kit). 
Table 6. Homogeneity of the $\mathrm{Cp}$ and $\mathrm{T}_{m}$ values of IRMM/IFCC-490. Vials were stored at $-70^{\circ} \mathrm{C}$ or at $-20^{\circ} \mathrm{C}$. Suspicious data are in bold.

\begin{tabular}{|c|c|c|c|c|c|c|c|}
\hline \multicolumn{8}{|c|}{ Homogeneity of $\mathrm{Cp}$ and $\mathrm{T}_{\mathrm{m}}$ values after storage at $-70^{\circ} \mathrm{C}$ or $-20^{\circ} \mathrm{C}$ for 2,4 or 8 weeks } \\
\hline $\begin{array}{c}\text { Vial } \\
\text { Number }\end{array}$ & $\begin{array}{l}\text { Storage time } \\
\text { at }-70^{\circ} \mathrm{C} \\
\text { (weeks) }\end{array}$ & $\begin{array}{c}\text { Replicate } \\
1\end{array}$ & $\begin{array}{c}\text { Replicate } \\
2\end{array}$ & $\begin{array}{c}\text { Average } \\
C p\end{array}$ & $s \mathrm{Cp}$ & $\begin{array}{c}\text { Average } \\
T_{m}\end{array}$ & $s \mathbf{T}_{\mathrm{m}}$ \\
\hline 9 & 8 & 14.82 & 14.64 & 14.73 & 0.13 & 59.61 & 0.01 \\
\hline 232 & 8 & 14.76 & 15.08 & 14.92 & 0.23 & 59.60 & 0.23 \\
\hline 469 & 8 & 14.61 & 14.98 & 14.80 & 0.26 & 59.46 & 0.00 \\
\hline 706 & 8 & 14.23 & 14.92 & 14.58 & 0.49 & 59.39 & 0.07 \\
\hline 945 & 8 & 14.50 & 15.07 & 14.79 & 0.40 & 59.42 & 0.08 \\
\hline 1166 & 8 & 14.60 & 14.82 & 14.71 & 0.16 & 59.44 & 0.11 \\
\hline $\begin{array}{c}\text { Vial } \\
\text { Number }\end{array}$ & $\begin{array}{l}\text { Storage time } \\
\text { at }-20^{\circ} \mathrm{C} \\
\text { (weeks) }\end{array}$ & $\begin{array}{c}\text { Replicate } \\
1\end{array}$ & $\begin{array}{c}\text { Replicate } \\
2\end{array}$ & $\begin{array}{c}\text { Average } \\
\mathrm{Cp}\end{array}$ & $s \mathrm{Cp}$ & $\begin{array}{c}\text { Average } \\
T_{m}\end{array}$ & $s T_{m}$ \\
\hline 81 & 2 & 14.37 & 14.77 & 14.57 & 0.28 & 59.53 & 0.13 \\
\hline 310 & 2 & 14.57 & 14.89 & 14.73 & 0.23 & 59.49 & 0.01 \\
\hline 550 & 2 & 14.67 & 14.80 & 14.74 & 0.09 & 59.43 & 0.02 \\
\hline 775 & 2 & 14.55 & 14.90 & 14.73 & 0.25 & 59.40 & 0.01 \\
\hline 1024 & 2 & 14.55 & 14.64 & 14.60 & 0.06 & 59.25 & 0.01 \\
\hline 1250 & 2 & 14.84 & 14.29 & 14.57 & 0.39 & 59.43 & 0.01 \\
\hline 85 & 4 & 14.87 & 14.70 & 14.79 & 0.12 & 59.56 & 0.01 \\
\hline 329 & 4 & 14.67 & 14.81 & 14.74 & 0.10 & 59.62 & 0.03 \\
\hline 560 & 4 & 14.64 & 14.80 & 14.72 & 0.11 & 59.54 & 0.09 \\
\hline 796 & 4 & 14.51 & 14.54 & 14.53 & 0.02 & 59.48 & 0.01 \\
\hline 1029 & 4 & 14.46 & 14.56 & 14.51 & 0.07 & 59.45 & 0.01 \\
\hline 1268 & 4 & 14.26 & 14.57 & 14.42 & 0.22 & 59.50 & 0.06 \\
\hline 91 & 8 & 14.75 & 14.71 & 14.73 & 0.03 & 59.61 & 0.19 \\
\hline 337 & 8 & 14.79 & 14.64 & 14.72 & 0.11 & 59.53 & 0.04 \\
\hline 584 & 8 & 13.49 & 14.53 & 14.01 & 0.74 & 59.57 & 0.06 \\
\hline 808 & 8 & 14.68 & 14.75 & 14.72 & 0.05 & 59.52 & 0.12 \\
\hline 1050 & 8 & 14.28 & 14.65 & 14.47 & 0.26 & 59.45 & 0.15 \\
\hline 1270 & 8 & 14.60 & 14.52 & 14.56 & 0.06 & 59.52 & 0.09 \\
\hline
\end{tabular}

In Table 6, the grand average and standard deviation of $\mathrm{Cp}$ is $14.64 \pm 0.18$ and of $T_{m}$ is $(59.49 \pm 0.09){ }^{\circ} \mathrm{C}$.

Scrutiny of the data: one suspicious datum was detected (in bold), and a trend was observed when it was not removed from the data set ( $t$-test; $t_{0.05,23}$, data not shown). The outlying datum was however retained in the evaluation, because here no distinction could be made between the influence of statistical variation and inhomogeneity of the batch.

All average $T_{m}$ values $\pm s$ of the individual samples (Table 6) are within the expected interval mentioned in the kit manual $(59 \pm 2.5){ }^{\circ} \mathrm{C}$. Therefore, all samples could be categorised as having the wildtype genotype. Moreover, since the batch has been prepared by dilution from a stock solution that had been sequenced and since all samples are liquid (frozen at $-20^{\circ} \mathrm{C}$ ), heterogeneity is unlikely to be found.

Analysis of the data by ANOVA on all Cp values is summarized in Table 7 (using MS Excel $^{\mathrm{TM}}$ Single Factor ANOVA). 
Table 7. Analysis of data from Table 6 using $M S$ Excel ${ }^{\mathrm{TM}}$ Single Factor ANOVA

\begin{tabular}{|l|r|r|r|}
\hline & SS & $v$ & $M S$ \\
\hline Among vials & 1.498 & 23 & 0.065 \\
\hline Within vials & 1.634 & 24 & 0.068 \\
\hline Total & 3.132 & 47 & \\
\hline
\end{tabular}

From Table 7 the repeatability of the procedure was calculated as $1.78 \%$, according to the procedures described in references [1-4]. The $s_{b b}$ could not be calculated as MS between groups was found to be inferior to MS within groups. The $\mathrm{u}_{\mathrm{bb}}{ }^{*}$ relative standard uncertainty was calculated as $0.68 \%$. The maximum relative standard uncertainty contribution to homogeneity was therefore $0.68 \%$.

A plot of the $\mathrm{Cp}$ values of the PCR products versus the sample number did not reveal a trend in the filling sequence (data not shown). The slope of the regression line was not statistically different from zero ( $t$-test, data not shown) and the data followed a normal and unimodal distribution (data not shown).

In conclusion, the batch IRMM/IFCC-490 produced is homogeneous and contains the wildtype sequence of a $609 \mathrm{bp}$ fragment of the human prothrombin gene. 


\section{Stability}

\subsection{Planning}

The stability studies have been assessed by isochronous measurements at time points of 2, 4 and 8 weeks for the short-term, and at 2, 4 and 6 months for the longterm study. The reference temperature was set at $-70^{\circ} \mathrm{C}$, corresponding to time point 0 .

\subsection{Short-term stability}

In order to assess the stability of the material, an indication of the pDNA quantity in each vial was investigated using the $C p$ values obtained after the PCR reaction in the LightCycler. Six different vials were tested in duplicate ( $5 \mu \mathrm{L}$ pDNA per PCR reaction) at each temperature. As the $\mathrm{Cp}$ values give an indication of the quantity of the pDNA remaining after storage, the melting temperatures allow genotyping of the DNA fragments (Tables 8.1 and 8.2).

Table 8.1: Short-term stability of IRMM/IFCC-490 stored for 8 weeks at $-70^{\circ} \mathrm{C}$

\begin{tabular}{|l|c|c|c|c|}
\hline & \multicolumn{2}{|c|}{ Cp values } & \multicolumn{2}{c|}{$\mathbf{T}_{\mathbf{m}}$ values } \\
\hline & Cp 1 & Cp 2 & $\mathbf{T}_{\mathbf{m}} \mathbf{1}$ & $\mathbf{T}_{\mathbf{m}} \mathbf{~}$ \\
\hline Sample 1 & 14.73 & 14.22 & 59.61 & 59.06 \\
\hline Sample 2 & 14.92 & 14.24 & 59.60 & 59.04 \\
\hline Sample 3 & 14.80 & 14.29 & 59.46 & 59.06 \\
\hline Sample 4 & 14.58 & 14.08 & 59.39 & 59.02 \\
\hline Sample 5 & 14.79 & 14.29 & 59.42 & 59.09 \\
\hline Sample 6 & 14.71 & 14.30 & 59.44 & 59.06 \\
\hline Average & 14.75 & 14.23 & 59.49 & 59.05 \\
\hline S & 0.11 & 0.08 & 0.09 & 0.02 \\
\hline
\end{tabular}

Cp 1 and $T_{m} 1$ represent respectively the averages of $C p$ and $T_{m}$ values of a duplicate measurement of samples stored at the reference temperature in the isochronous study investigating the stability at $-20^{\circ} \mathrm{C}, 4^{\circ} \mathrm{C}$ and $18^{\circ} \mathrm{C}$. Cp 2 and $\mathrm{T}_{\mathrm{m}} 2$ represent respectively the averages of $\mathrm{Cp}$ and $\mathrm{T}_{m}$ values of a duplicate measurement of samples stored at the reference temperature in the isochronous study investigating the stability at $40^{\circ} \mathrm{C}$. The grand average and standard deviation of the $\mathrm{Cp}$ values and $\mathrm{T}_{\mathrm{m}}$ values of samples stored at $-70^{\circ} \mathrm{C}$ is respectively $\mathrm{Cp} 1=14.75 \pm 0.11, \mathrm{Cp} 2=$ $14.23 \pm 0.08$ and $\mathrm{T}_{\mathrm{m}} 1=(59.49 \pm 0.09){ }^{\circ} \mathrm{C}, \mathrm{T}_{\mathrm{m}} 2=(59.05 \pm 0.02){ }^{\circ} \mathrm{C}$. 
The samples of Table 8.1 were analysed simultaneously with those in Table 8.2 as they represented the samples stored at the reference temperature in the stability study.

Table 8.2: Short-term stability of IRMM/IFCC-490 stored for 2, 4 and 8 weeks at four different temperatures. Suspicious data are in bold

\begin{tabular}{|c|c|c|c|c|}
\hline & & \multicolumn{3}{|c|}{ Cp values at $t$ (week) } \\
\hline & $\begin{array}{l}\text { Temperature } \\
{ }^{\circ} \mathrm{C}\end{array}$ & $t=2$ & $t=4$ & $t=8$ \\
\hline Sample 1 & -20 & 14.57 & 14.79 & 14.73 \\
\hline Sample 2 & & 14.73 & 14.74 & 14.72 \\
\hline Sample 3 & & 14.74 & 14.72 & 14.01 \\
\hline Sample 4 & & 14.73 & 14.53 & 14.72 \\
\hline Sample 5 & & 14.60 & 14.51 & 14.47 \\
\hline Sample 6 & & 14.57 & 14.42 & 14.56 \\
\hline Average & & 14.65 & 14.62 & 14.53 \\
\hline$S$ & & 0.08 & 0.15 & 0.28 \\
\hline Sample 1 & 4 & 14.71 & 14.79 & 14.64 \\
\hline Sample 2 & & 15.04 & 14.68 & 14.83 \\
\hline Sample 3 & & 14.50 & 14.89 & 14.60 \\
\hline Sample 4 & & 15.01 & 14.71 & 14.92 \\
\hline Sample 5 & & 14.48 & 14.79 & 14.46 \\
\hline Sample 6 & & 14.85 & 14.70 & 14.65 \\
\hline Average & & 14.76 & 14.76 & 14.68 \\
\hline S & & 0.25 & 0.08 & 0.17 \\
\hline Sample 1 & 18 & 13.98 & 13.48 & 13.03 \\
\hline Sample 2 & & 14.06 & 13.70 & 13.17 \\
\hline Sample 3 & & 13.92 & 13.67 & 12.94 \\
\hline Sample 4 & & 14.44 & 13.69 & 13.32 \\
\hline Sample 5 & & 14.30 & 13.31 & 13.02 \\
\hline Sample 6 & & 13.94 & 13.58 & 12.86 \\
\hline Average & & 14.10 & 13.57 & 13.06 \\
\hline$S$ & & 0.21 & 0.15 & 0.17 \\
\hline Sample 1 & 40 & 10.62 & 10.55 & 11.13 \\
\hline Sample 2 & & 10.46 & 10.65 & 10.80 \\
\hline Sample 3 & & 10.7 & 10.28 & 10.87 \\
\hline Sample 4 & & 9.39 & 10.54 & 10.87 \\
\hline Sample 5 & & 10.68 & 10.38 & 10.80 \\
\hline Sample 6 & & 10.61 & 10.41 & 10.86 \\
\hline Average & & 10.41 & 10.47 & 10.89 \\
\hline$S$ & & 0.50 & 0.14 & 0.12 \\
\hline
\end{tabular}


The grand average and standard deviation of the $\mathrm{Cp}$ values of the vials stored at -20 ${ }^{\circ} \mathrm{C}$ is $\mathrm{Cp}=14.60 \pm 0.18, \mathrm{Cp}=14.73 \pm 0.17$ at $4{ }^{\circ} \mathrm{C}, \mathrm{Cp}=13.58 \pm 0.47$ at $18{ }^{\circ} \mathrm{C}$, and $\mathrm{Cp}=10.59 \pm 0.36$ at $40^{\circ} \mathrm{C}$ (Table 8.2).

Scrutiny of the data: one suspicious datum (indicated in bold) was detected with a discrepancy of more than $50 \%$ to the average between vials at the corresponding time point and temperature. The outlying datum at $-20{ }^{\circ} \mathrm{C}$ was retained in the evaluation, because here no distinction can be made between the influence of statistical variation and inhomogeneity of the batch.

For the four temperatures the slope of the regression line and its uncertainty were calculated and tested for statistical significance (Figure 4).

At all temperatures, the slope of the regression line was significantly different from 0 , even when removing the suspicious datum ( $t$-test; $t_{0.05,23}$, data not shown), but as the concentration of the material was slightly increasing with time (i.e. decreasing $\mathrm{Cp}$ values), the regression line was technically insignificant.

The recovery of the material was increasing, especially after storage at $40{ }^{\circ} \mathrm{C}$, due to evaporation of the solution with storage time. However, the reconstitution of the dried material (after 8 weeks at $40^{\circ} \mathrm{C}$ ) in a $50 \mu \mathrm{L}$ volume of water showed that the pDNA sequence was still present in sufficient amount to allow correct genotyping (Table 9).
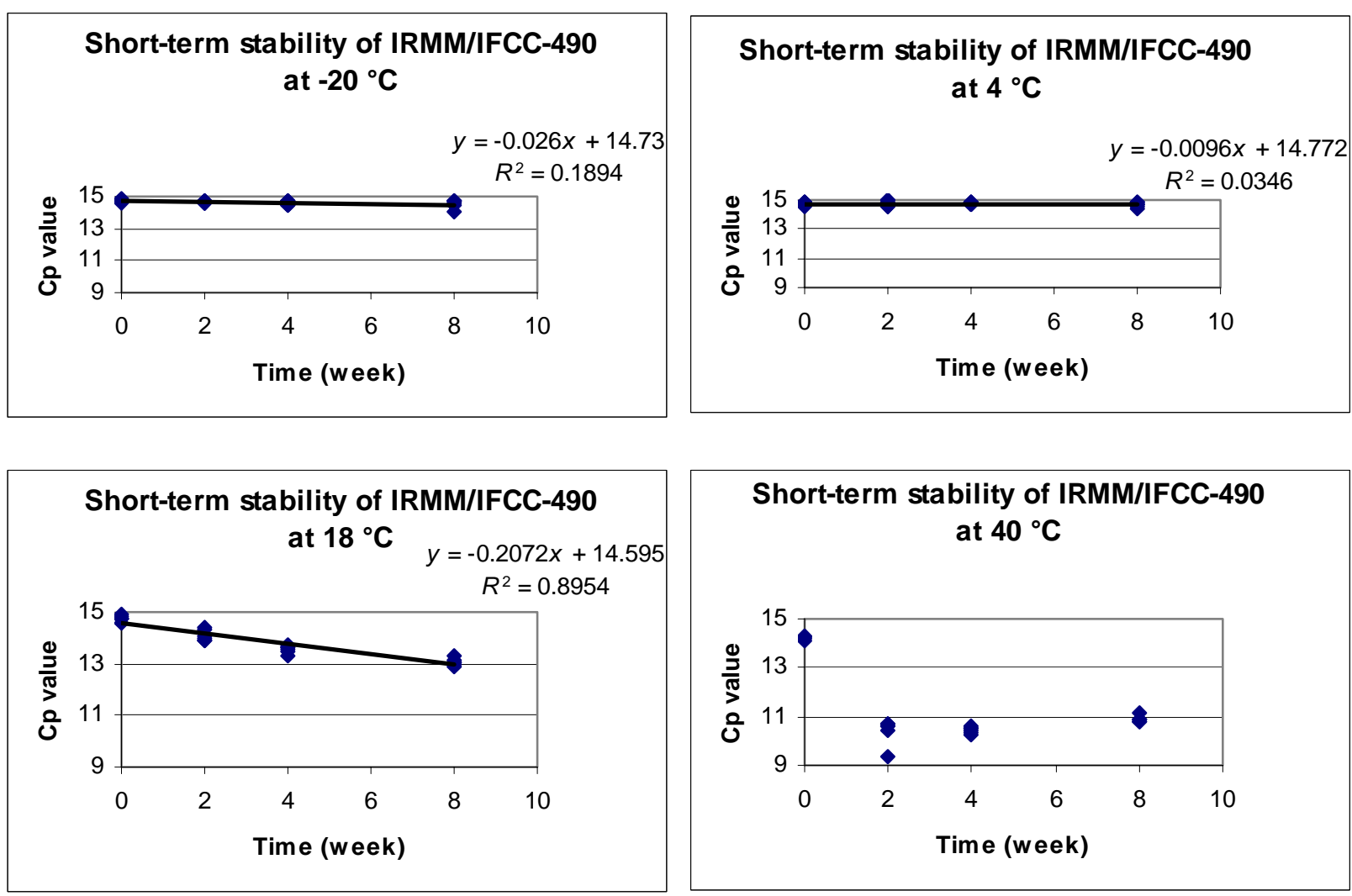

Figure 4. Slopes of the regression lines of IRMM/IFCC-490 during the shortterm stability study $\left(0,2,4\right.$ and 8 weeks at $-20^{\circ} \mathrm{C}, 4^{\circ} \mathrm{C}, 18^{\circ} \mathrm{C}$ and $\left.40^{\circ} \mathrm{C}\right)$ 
Table 9: Melting temperature $I^{\circ} \mathrm{C}$ of short-term stability samples of IRMM/IFCC-

490 stored for 2,4 and 8 weeks at $-20^{\circ} \mathrm{C}, 4^{\circ} \mathrm{C}, 18{ }^{\circ} \mathrm{C}$ and $40^{\circ} \mathrm{C}$

\begin{tabular}{|c|c|c|c|c|}
\hline & \multicolumn{4}{|c|}{$T_{m}$ values at $t$ (week) } \\
\hline & $\begin{array}{l}\text { Temperature } \\
{ }^{\circ} \mathrm{C}\end{array}$ & $t=2$ & $t=4$ & $t=8$ \\
\hline Sample 1 & -20 & 59.53 & 59.56 & 59.61 \\
\hline Sample 2 & & 59.49 & 59.62 & 59.53 \\
\hline Sample 3 & & 59.43 & 59.54 & 59.57 \\
\hline Sample 4 & & 59.40 & 59.48 & 59.52 \\
\hline Sample 5 & & 59.25 & 59.45 & 59.45 \\
\hline Sample 6 & & 59.43 & 59.50 & 59.52 \\
\hline Average & & 59.42 & 59.52 & 59.53 \\
\hline$s$ & & 0.10 & 0.06 & 0.05 \\
\hline Sample 1 & 4 & 59.57 & 59.63 & 59.67 \\
\hline Sample 2 & & 59.66 & 59.61 & 59.66 \\
\hline Sample 3 & & 59.5 & 59.57 & 59.64 \\
\hline Sample 4 & & 59.47 & 59.53 & 59.61 \\
\hline Sample 5 & & 59.41 & 59.5 & 59.46 \\
\hline Sample 6 & & 59.51 & 59.47 & 59.52 \\
\hline Average & & 59.52 & 59.55 & 59.59 \\
\hline$s$ & & 0.09 & 0.08 & 0.13 \\
\hline Sample 1 & 18 & 59.62 & 59.59 & 59.47 \\
\hline Sample 2 & & 59.78 & 59.70 & 59.49 \\
\hline Sample 3 & & 59.64 & 59.61 & 59.49 \\
\hline Sample 4 & & 59.57 & 59.44 & 59.47 \\
\hline Sample 5 & & 59.58 & 59.43 & 59.36 \\
\hline Sample 6 & & 59.52 & 59.45 & 59.51 \\
\hline Average & & 59.62 & 59.53 & 59.46 \\
\hline$s$ & & 0.10 & 0.12 & 0.11 \\
\hline Sample 1 & 40 & 59.13 & 58.99 & 59.02 \\
\hline Sample 2 & & 59.06 & 58.99 & 58.95 \\
\hline Sample 3 & & 59.06 & 58.95 & 58.98 \\
\hline Sample 4 & & 59.02 & 58.97 & 58.97 \\
\hline Sample 5 & & 58.95 & 58.95 & 58.96 \\
\hline Sample 6 & & 58.97 & 58.94 & 59.06 \\
\hline Average & & 59.03 & 58.96 & 58.99 \\
\hline$s$ & & 0.13 & 0.15 & 0.14 \\
\hline
\end{tabular}

In Table 9, each value represents an average of a duplicate. The average of six samples for each combination time/temperature shows that all $T_{m}$ values (Table 9) 
are within the interval of the expected value and standard deviation mentioned in the kit manual ((59 \pm 2.5$){ }^{\circ} \mathrm{C}$, Roche Diagnostics, Vilvoorde, Belgium).

Therefore, all samples could be categorised as having the wildtype genotype.

In conclusion, the batch IRMM/IFCC-490 is stable at $-20^{\circ} \mathrm{C}$ and contains the wildtype sequence of a $609 \mathrm{bp}$ fragment of human prothrombin, that can still be identified in samples which were stored at higher temperature such as $18{ }^{\circ} \mathrm{C}$ and $40{ }^{\circ} \mathrm{C}$ for up to 8 weeks.

\subsection{Long-term stability}

At temperatures of $18{ }^{\circ} \mathrm{C}$ and $40{ }^{\circ} \mathrm{C}$, evaporation occurred already after 2 months, therefore the long-term stability was not investigated at these temperatures.

The long-term stability study was performed at $-20{ }^{\circ} \mathrm{C}$ and at $4{ }^{\circ} \mathrm{C}$ for time periods of 2, 4 and 6 months (Table 10.2). Six different vials were tested in triplicate $(5 \mu \mathrm{L}$ pDNA per PCR reaction) at each temperature by real-time PCR and the triplicate average is displayed in Tables 10.1 and 10.2. As two different instruments (ABI Prism $7900 \mathrm{HT}$ and ABI Prism 7700) were used to analyse the samples, an internal control was used on each plate to be able to compare the data, and therefore the average $\mathrm{Ct}$ value for each sample was normalised by the average value of the triplicate measurements of the control on each plate, giving $\mathrm{Ct}^{\star}$ values. The average Ct values obtained using the ABI Prism 7700 instrument are displayed in bold. The average $\mathrm{Ct}$ value and standard deviation of the internal control (pDNA at $0.02 \mathrm{ng} / \mu \mathrm{L}$ ) was $15.72 \pm 0.17$ and $16.34 \pm 0.09$ using the $\mathrm{ABI} 7900 \mathrm{HT}$ and $\mathrm{ABI}$ Prism 7700 instruments, respectively.

Table 10.1. Long-term stability of IRMM/IFCC-490 stored for 6 months at $-70^{\circ} \mathrm{C}$

\begin{tabular}{|c|c|c|c|c|}
\hline & & \multicolumn{2}{|c|}{ Ct values } & \multirow{2}{*}{$\begin{array}{c}\mathrm{T}_{\mathrm{m}} \text { values } \\
{ }^{\circ} \mathrm{C}\end{array}$} \\
\hline & $\begin{array}{l}\text { Temperature } \\
{ }^{\circ} \mathrm{C}\end{array}$ & $\mathbf{C t}$ & $\mathbf{C t}^{*}$ & \\
\hline Sample 1 & -70 & 15.65 & 1.00 & 59.12 \\
\hline Sample 2 & & 15.62 & 0.99 & 59.18 \\
\hline Sample 3 & & 15.41 & 0.98 & 59.21 \\
\hline Sample 4 & & 15.61 & 0.99 & 59.16 \\
\hline Sample 5 & & 15.57 & 0.99 & 59.15 \\
\hline Sample 6 & & 15.64 & 0.99 & 59.13 \\
\hline Average & & 15.58 & 0.99 & 59.16 \\
\hline$s$ & & 0.09 & 0.01 & 0.08 \\
\hline \multicolumn{2}{|c|}{ Control ABI 7900HT } & 15.72 & - & - \\
\hline
\end{tabular}

The data of Table 10.1 were analysed simultaneously with those in Table 10.2 as they represented the samples stored at the reference temperature. The grand 
average and standard deviation of the $\mathrm{Ct}^{\star}$ values and $\mathrm{T}_{\mathrm{m}}$ values of samples stored at $-70{ }^{\circ} \mathrm{C}$ is respectively $0.99 \pm 0.01$ and $(59.16 \pm 0.08){ }^{\circ} \mathrm{C}$. The $T_{m}$ values of Table 10.1 constitute the values obtained at the reference temperature for samples analysed in Table 11.

Table 10.2. Long-term stability of IRMM/IFCC-490 stored for 2, 4 and 6 months at two different temperatures. The average $\mathrm{Ct}$ values obtained using the $\mathrm{ABI}$ Prism 7700 instrument are displayed in bold

\begin{tabular}{|c|c|c|c|c|c|c|c|}
\hline & & \multicolumn{6}{|c|}{ Ct values at $t$ (month) } \\
\hline & \multirow{2}{*}{$\begin{array}{l}\text { Temperature } \\
{ }^{\circ} \mathrm{C}\end{array}$} & \multicolumn{2}{|c|}{$t=2$} & \multicolumn{2}{|c|}{$t=4$} & \multicolumn{2}{|c|}{$t=6$} \\
\hline & & Ct & $\mathbf{C t}^{*}$ & Ct & $\mathbf{C t}^{*}$ & Ct & $\mathbf{C t}^{*}$ \\
\hline Sample 1 & -20 & 15.40 & 0.98 & 15.59 & 0.99 & 15.66 & 1.00 \\
\hline Sample 2 & & 15.64 & 0.99 & 15.55 & 0.99 & 15.44 & 0.98 \\
\hline Sample 3 & & 15.54 & 0.99 & 15.44 & 0.98 & 15.71 & 1.00 \\
\hline Sample 4 & & 15.49 & 0.99 & 15.65 & 1.00 & 15.66 & 1.00 \\
\hline Sample 5 & & 15.58 & 0.99 & 15.54 & 0.99 & 15.62 & 0.99 \\
\hline Sample 6 & & 15.61 & 0.99 & 15.74 & 1.00 & 15.59 & 0.99 \\
\hline Average & & 15.54 & 0.99 & 15.58 & 0.99 & 15.61 & 0.99 \\
\hline$s$ & & 0.09 & 0.01 & 0.10 & 0.01 & 0.09 & 0.01 \\
\hline \multicolumn{2}{|c|}{ Control ABI 7900HT } & 15.72 & & 15.72 & & 15.72 & \\
\hline Sample 1 & 4 & 15.52 & 0.99 & 16.26 & 0.99 & 16.06 & 0.98 \\
\hline Sample 2 & & 15.30 & 0.97 & 16.23 & 0.99 & 16.17 & 0.98 \\
\hline Sample 3 & & 15.42 & 0.98 & 16.24 & 0.99 & 16.1 & 0.99 \\
\hline Sample 4 & & 15.38 & 0.98 & 16.17 & 0.98 & 16.22 & 0.98 \\
\hline Sample 5 & & 15.44 & 0.98 & 16.18 & 0.99 & 15.98 & 0.99 \\
\hline Sample 6 & & 15.37 & 0.98 & 16.36 & 1.00 & 16.03 & 0.99 \\
\hline Average & & 15.41 & 0.98 & 16.18 & 0.99 & 16.09 & 0.98 \\
\hline$s$ & & 0.08 & 0.01 & 0.08 & 0.01 & 0.08 & 0.01 \\
\hline \multicolumn{2}{|c|}{ Control ABI 7900HT } & 15.72 & & - & & - & \\
\hline \multicolumn{2}{|c|}{ Control ABI 7700} & - & & 16.34 & & 16.34 & \\
\hline
\end{tabular}

Scrutiny of the data: no suspicious data were detected.

For the two temperatures, the slope of the regression line using the $\mathrm{Ct}^{\star}$ values (Figures $5 \mathrm{~A}$ and $5 \mathrm{~B}$ ) and its uncertainty were calculated and tested for statistical significance ( $t$-test; $t_{0.05,22}$ ). In both cases, the slope was not significantly different from zero (data not shown). 
Figure $5 \mathrm{~A}$ : at $-20^{\circ} \mathrm{C}$

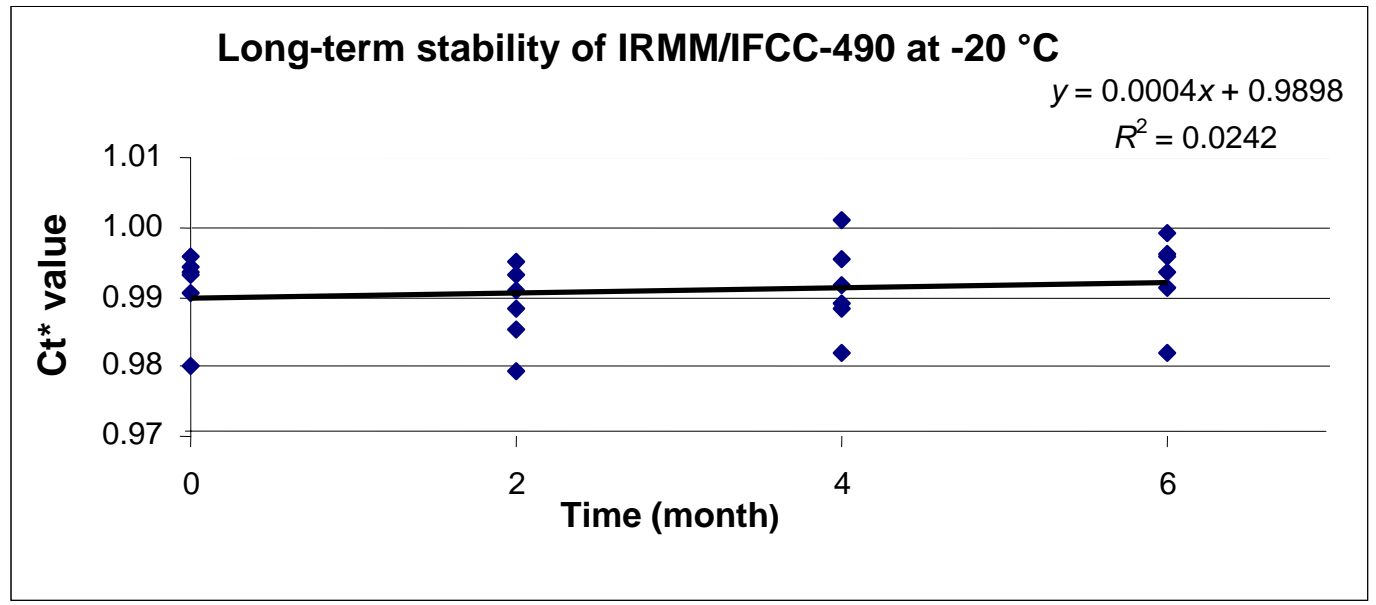

Figure 5B: at $4^{\circ} \mathrm{C}$

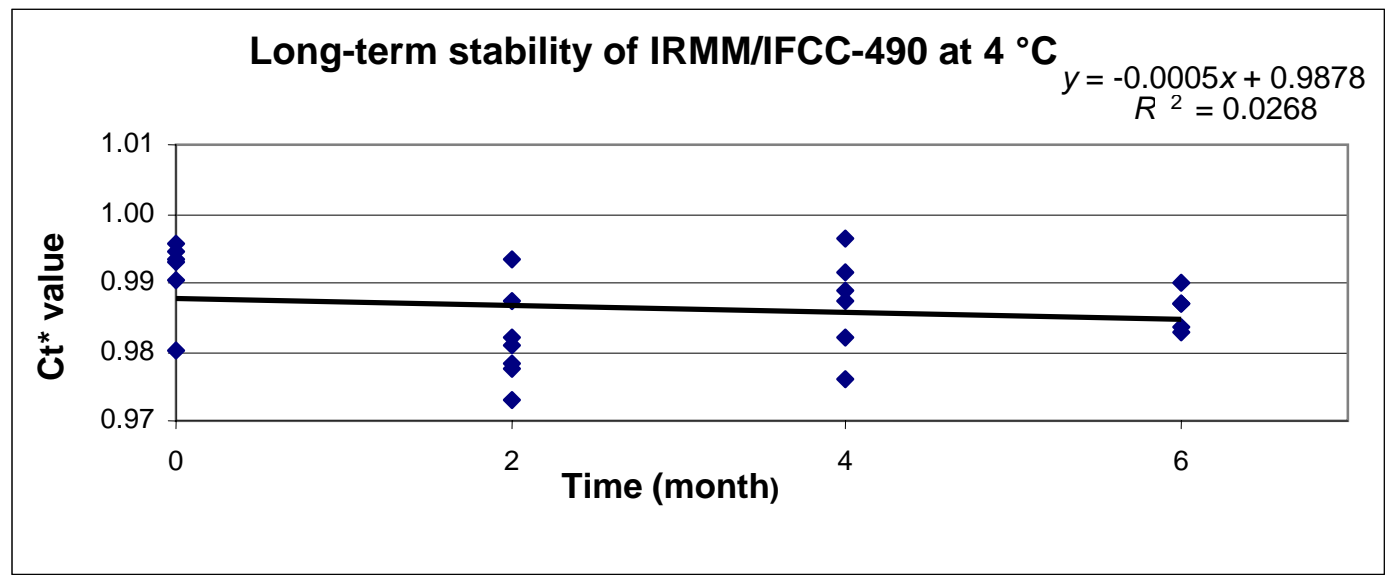

Figure 5. Slopes of the regression lines of IRMM/IFCC-490 during long-term stability study $\left(0,2,4\right.$ and 6 months at $-20^{\circ} \mathrm{C}(\mathrm{A})$ and at $\left.4{ }^{\circ} \mathrm{C}(\mathrm{B})\right)$

Additionally, the melting temperatures of the PCR fragments were recorded for genotyping purposes. All $T_{m}$ values of the individual samples (Tables 10.1 and 10.2) are within the interval of the expected value mentioned in the kit manual $\left(59 \pm 2.5^{\circ} \mathrm{C}\right.$, Roche Diagnostics, Vilvoorde, Belgium).

Therefore, all samples could be categorised as having the wildtype genotype.

In conclusion, the batch of IRMM/IFCC-490 is stable for 6 months at $-20^{\circ} \mathrm{C}$ and at 4 ${ }^{\circ} \mathrm{C}$ and contains the wildtype sequence of a 609 bp fragment of the human prothrombin gene. 
Table 11: Melting temperature $I^{\circ} \mathrm{C}$ of long-term stability samples of IRMM/IFCC490 stored for 2,4 and 6 months at $-20^{\circ} \mathrm{C}$ and at $4{ }^{\circ} \mathrm{C}$

\begin{tabular}{|l|l|c|c|c|}
\hline & & \multicolumn{3}{|c|}{$T_{\mathrm{m}}$ values at t (month) } \\
\hline & $\begin{array}{l}\text { Temperature } \\
{ }^{\circ} \mathrm{C}\end{array}$ & $\boldsymbol{t}=\mathbf{2}$ & $\boldsymbol{t}=\mathbf{4}$ & $\boldsymbol{t}=\mathbf{6}$ \\
\hline Sample 1 & -20 & 59.16 & 59.04 & 59.13 \\
\hline Sample 2 & & 59.12 & 59.18 & 59.22 \\
\hline Sample 3 & & 59.17 & 59.28 & 59.19 \\
\hline Sample 4 & & 59.12 & 59.05 & 59.17 \\
\hline Sample 5 & & 59.10 & 59.06 & 59.14 \\
\hline Sample 6 & & 59.12 & 59.06 & 59.12 \\
\hline Average & & 59.13 & 59.11 & 59.16 \\
\hline S & & 0.03 & 0.10 & 0.04 \\
\hline Sample 1 & 4 & 59.82 & 59.58 & 60.45 \\
\hline Sample 2 & & 59.85 & 59.50 & 60.34 \\
\hline Sample 3 & & 59.75 & 59.53 & 60.55 \\
\hline Sample 4 & & 59.75 & 59.48 & 60.49 \\
\hline Sample 5 & & 59.72 & 59.57 & 60.31 \\
\hline Sample 6 & & 59.76 & 59.46 & 60.21 \\
\hline Average & & 59.77 & 59.52 & 60.39 \\
\hline S & & 0.05 & 0.05 & 0.12 \\
\hline
\end{tabular}




\section{Batch characterisation}

After plasmid construction and production, the cloned human prothrombin (Factor II) fragment in pUC18 plasmid was sequenced using DTCS protocol [10] (see [6]).

\subsection{Sequencing}

The $609 \mathrm{bp}$ fragment of the human prothrombin was sequenced using sequencing forward primers M13-F (P-0419) and Factor II-F (P-0579) and reverse primers Factor II-R (P-0580) and M13-R (P-0289), and DTCS protocol (Table 5, [10]).

Forward and reverse sequences matched, indicating a very low probability of misreadings $\left(p<3 \times 10^{-6}\right)$. As there was no error in the sequencing of the insert, the misreading probability was calculated as to be inferior to $1 / 609$ for the forward sequencing reactions, and inferior to $1 / 609$ when backward sequencing reactions were performed. Therefore, the total misreadings probability in the certified sequence was calculated as inferior to $1 / 609 \times 1 / 609$.

The analysed fragment of prothrombin DNA sequence showed $100 \%$ homology with the reported DNA sequence of the prothrombin gene (GeneBank Accession number M17262), confirming the identity of produced pDNA (Figure 6). This sequence was also confirmed by sequencing using the U-20 universal primer and an $A B I 377$ instrument by Eurogentec (Liège, Belgium).

\begin{tabular}{|c|c|c|c|}
\hline Query & 1 & GCACAGACGGCTGTTCTCTTTC & \\
\hline Subject & 26302 & gcacagacggctgttctcttc & \\
\hline Query & 23 & AAGGTTACAAGCCTGATGAAGGGAAACGAGGGGATGCCTGTGAAGGTGAC & 72 \\
\hline Subject & 26324 & aaggttacaagcctgatgaagggaaacgaggggatgcctgtgaaggtgac & 26373 \\
\hline Query & 73 & AGTGGGGGACCCTTTGTCATGAAGGTAAGCTTCTCTAAAGCCCAGGGCCT & 122 \\
\hline Subject & 26374 & agtgggggaccctttgtcatgaaggtaagcttctctaaagcccagggcct & 26423 \\
\hline Query & 123 & $\begin{array}{l}\text { GGTGAACACATCTTCTGGGGGTGGGGAGAAACTCTAGTATCTAGAAACAG } \\
\text { (a) }\end{array}$ & 172 \\
\hline Subject & 26424 & ggtgaacacatcttctgggggtggggagaactctagtatctagaaacag & 26473 \\
\hline Query & 173 & CCTTGAACTTGACTCTATTGGAA & 222 \\
\hline Subject & 26474 & ttgcctggcagaggaatactgatgtgaccttgaacttgactctattggaa & 26523 \\
\hline Query & 223 & CCTTTAACAACCGCTGGTATCAAATG & 272 \\
\hline Subject & 26524 & acctcatcttcttcttcagagcccctttaacaaccgctggtatcaaatg & 26573 \\
\hline Query & 273 & TATGGCTT & 322 \\
\hline
\end{tabular}




\begin{tabular}{|c|c|c|c|}
\hline Subject & 26574 & ggcatcgtctcatggggtgaaggctgtgaccgggatgggaaatatggctt & 26623 \\
\hline Query & 323 & $\begin{array}{l}\text { CTACACACATGTGTTCCGCCTGAAGAAGTGGATACAGAAGGTCATTGATC } \\
\text { (a) }\end{array}$ & 372 \\
\hline Subject & 26624 & ctacacacatgtgttccgcctgaagaagtggatacagaaggtcattgatc & 26673 \\
\hline Query & 373 & AGTTTGGAGAGTAGGGGGCCACTCATATTCTGGGCTCCTGGAACCAATCC & 422 \\
\hline Subject & 26674 & agtttggagagtagggggccactcatattctgggctcctggaaccaatcc & 26723 \\
\hline Query & 423 & CGTGAAAGAATTATTTTTGTGTTTCTAAAACTATGGTTCCCAATAAAAGT & 472 \\
\hline & 26724 & cgtgaaagaattatttttgtgtttctaaactatggttcccaataaaagt & 26773 \\
\hline Query & 473 & 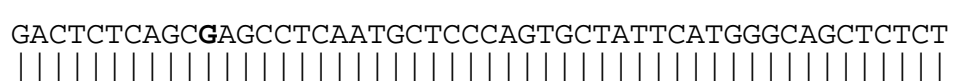 & 522 \\
\hline Subject & 26774 & gactctcagcgagcctcaatgctcccagtgctattcatgggcagctctct & 26823 \\
\hline Query & 523 & CAGTAATACTACTGGATAAAGAAGACTTAAGAATC & 572 \\
\hline Subject & 26824 & gggctcaggaagagccagtaatactactggataaagaagacttaagaatc & 26873 \\
\hline Query & 573 & $\begin{array}{l}\text { CACCACCTGGTGCACGCTGGTAGTCCGAGCACTCGGG } \\
\text { CACA The }\end{array}$ & \\
\hline Subject & 26874 & caccacctggtgcacgctggtagtccgagcactcggg & \\
\hline
\end{tabular}

Figure 6. Comparison of the homology of the DNA sequence of the $609 \mathrm{bp}$ fragment of human prothrombin obtained from IRMM/IFCC-490 production batch (Query) with the reported DNA sequence (Subject) of the prothrombin gene (GeneBank accession number M17262) using BLAST 2 sequences on www.ncbi.nlm.nih.gov

In conclusion, the correct wildtype sequence was confirmed for the IRMM/IFCC-490 material. The sequencing method is the most robust and straightforward one to determine a DNA sequence, but requires a rather large minimum amount of DNA and is relatively time consuming. When only a lower DNA quantity is available, it can only be detected by using PCR-based methods such as real-time PCR, hybridisation techniques and DNA profiling. 


\section{Validation}

\subsection{Field study design and results}

The performance of the material was tested in a field study by 7 diagnostic laboratories using 4 different methods routinely for the G20210A prothrombin mutation detection (Table 12). A total of eight vials labeled from $\mathrm{A}$ to $\mathrm{H}$ was sent to each laboratory. Each set of vials contained two vials of each genotype: IRMM/IFCC490 (wildtype sequence), IRMM/IFCC-491 (G20210A mutant sequence) or IRMM/IFCC-492 (heterozygous wildtype/G20210A mutant sequence generated by mixing of equal volumes of identical mass concentrations of IRMM/IFCC-490 and IRMM/IFCC-491), and two vials without DNA (Blank). The testing laboratories were provided with the study protocol, the terms to be used for reporting and the results reporting form, but had no information on the expected results or identity of the individual samples. All laboratories were asked to describe in detail the method used.

Table 12: Field study of IRMM/IFCC-490, -491 and -492 materials (Y: Yes, N: No)

\begin{tabular}{|c|c|c|c|c|c|c|}
\hline Lab & Method & Type & & Correctness & f genotyping & \\
\hline & & & $\begin{array}{l}\text { Homozygous } \\
\text { wildtype }\end{array}$ & $\begin{array}{l}\text { Homozygous } \\
\text { mutant }\end{array}$ & $\begin{array}{l}\text { Heterozygous } \\
\text { wildtype/G20210A } \\
\text { mutant }\end{array}$ & Blank \\
\hline 1 & PCR & LightCycler & $Y$ & $Y$ & $Y$ & $Y$ \\
\hline 2 & $\begin{array}{l}\text { Multiplex } \\
\text { PCR }\end{array}$ & Home brewed & Y & $Y$ & $Y$ & Y \\
\hline 3 & $\begin{array}{l}\text { Multiplex } \\
\text { PCR }\end{array}$ & Home brewed & Y & $Y$ & $Y$ & $Y$ \\
\hline 4 & PCR & LightCycler & $\mathrm{Y}$ & $Y$ & $Y$ & $\mathrm{Y}$ \\
\hline 5 & RFLP1 & Home brewed & $\mathrm{Y}$ & $Y$ & $Y$ & $Y$ \\
\hline 5 & PCR & LightCycler & $\mathrm{Y}$ & $Y$ & $\mathrm{Y}$ & $\mathbf{N}$ \\
\hline 6 & RFLP2 & Home brewed & $\mathrm{Y}$ & $Y$ & $Y$ & $\mathrm{Y}$ \\
\hline 7 & PCR & Rotor-Gene & Y & $Y$ & $Y$ & Y \\
\hline
\end{tabular}

In Table 12, each $\mathrm{Y}$ or $\mathrm{N}$ represents agreement on the two samples that have been tested by the corresponding laboratory. All DNA-containing samples were genotyped correctly. Two vials without DNA were misclassified (one bold $\mathbf{N}$ representing the agreement on two blank samples). These data were reported as dubious by lab number 5 using the LightCycler method due to the unusually weak intensity of the signal generated. The same samples, tested with the RFLP method by the same laboratory, were correctly identified as blank samples. The use of two different methods by lab 5 was agreed before starting the study. In laboratory 3 , the samples were diluted 1:20 before use, but the dilution of the material did not influence the genotyping results. 
All vials containing pDNA (wildtype, mutant or heterozygous wildtype/G20210A mutant) were genotyped correctly and unambiguously demonstrating that the material is fit for its intended purpose.

\subsection{Methods}

Roche LightCycler: LightCycler technology from Roche, using the Factor II (prothrombin) G20210A kit and LightCycler 1.5 instrument [5].

Multiplex mutagenic separated PCR: derived from Endler et al. [11].

Thermal PCR program: $35 \times$ cycles of $95{ }^{\circ} \mathrm{C}$ for $1 \mathrm{~min} ; 54{ }^{\circ} \mathrm{C}$ for $2 \mathrm{~min} ; 72{ }^{\circ} \mathrm{C}$ for 1 min; then hold at $4{ }^{\circ} \mathrm{C}$. Primers used are described hereafter:

\begin{tabular}{|l|l|}
\hline Forward (wildtype), F2G & 5'-CAC TGG GAG CAT TGA GGC GC -3' \\
\hline Forward (mutant), F2A & 5'-ATG AAT AGT AAT GGG AGC ATT GAG GAT T -3' \\
\hline Reverse & 5'- ATG TGT TCC GCC TGA AGA AGT GGA -3' \\
\hline
\end{tabular}

PCR products $(6 \mu \mathrm{L})$ were loaded on Spreadex EL 600 gels, run at $120 \mathrm{~V}$ for $2 \mathrm{~h}$, then for $20 \mathrm{~min}$ at $40{ }^{\circ} \mathrm{C}$ to $50^{\circ} \mathrm{C}$. Staining of the gels performed with $1: 20000$ diluted SYBRGreen for 30 min at room temperature.

RFLP1: Derived from Poort el al. [12]

Thermal PCR program: $95^{\circ} \mathrm{C}$ for $4 \mathrm{~min}, 32 \times$ cycles of $94{ }^{\circ} \mathrm{C}$ for $1 \mathrm{~min}$; $55^{\circ} \mathrm{C}$ for 1 $\min ; 67^{\circ} \mathrm{C}$ for $1 \mathrm{~min}$; then hold at $4{ }^{\circ} \mathrm{C}$. Primers used are described hereafter:

\begin{tabular}{|l|l|}
\hline Forward & 5'-TCT AGA AAC AGT TGC CTG GC-3' \\
\hline Reverse & 5'-ATA GCA CTG GGA GCA TTG AAG C -3' \\
\hline
\end{tabular}

PCR products are digested with HindIII restriction enzyme and loaded on agarose gel for electrophoresis.

RFLP2: Derived from Danneberg el al. [13]

Thermal PCR program: $94{ }^{\circ} \mathrm{C}$ for $5 \mathrm{~min}, 35 \mathrm{x}$ cycles of $93^{\circ} \mathrm{C}$ for $1 \mathrm{~min} ; 58^{\circ} \mathrm{C}$ for 1 min; $72{ }^{\circ} \mathrm{C}$ for $1 \mathrm{~min} ; 72{ }^{\circ} \mathrm{C}$ for $3 \mathrm{~min}$, then hold at $4{ }^{\circ} \mathrm{C}$. Primers used are described hereafter:

\begin{tabular}{|l|l|}
\hline Forward & 5'-GCA CAG ACG GTG TTC TCT T -3' \\
\hline Reverse & 5'-ATA GCA CTG GGA GCA TTG AAG C -3' \\
\hline
\end{tabular}


PCR products are digested with HindIII restriction enzyme and loaded on agarose gel for electrophoresis.

Rotor-Gene real-time PCR analysis software 6.0: Factor II kit from Nuclear Laser Medicine, Settala, IT. 


\section{Traceability of gene fragment}

The sequence of the $609 \mathrm{bp}$ human prothrombin gene fragment inserted in the pUC18 vector to generate the IRMM/IFCC-490 material was assessed by the CEQ Dye Terminator Cycle Sequencing (including a pUC18 control plasmid) and confirmed by external sequencing by Eurogentec (Liège, Belgium) using the U-20 universal primer and the $\mathrm{ABI}$ Prism 377 instrument. The sequence identity is traceable to PCR-based sequencing methods with very low probability of misreadings $\left(p<3 \times 10^{-6}\right)$. 


\section{Value assignment}

There is no numerical value assignment for this reference material. The sequence at position 20210 is a G, and certified by using 2 different sequencing procedures. The uncertainty can be expressed as a probability that two procedures, independently performed in different laboratories, generated identical results by forward and backward sequencing $\left(p<3 \times 10^{-6}\right)$. The misreading probability was calculated as to be inferior to $1 / 609$ for the forward sequencing reaction, and inferior to $1 / 609$ when backward sequencing was performed. Therefore, the total misreadings probability was calculated as inferior to $1 / 609 \times 1 / 609$.

Uncertainties related to homogeneity and stability studies did not jeopardize the presence of the target sequence, and it could be demonstrated that even after 8 weeks at $18{ }^{\circ} \mathrm{C}$ and $40{ }^{\circ} \mathrm{C}$ the material could still be used in genotyping experiments without impact on the performance of the qualitative methods applied for nucleotide identification. 


\section{Instructions for use}

\subsection{Dispatch}

Dispatch to the customer should be done with cooling elements. Upon receipt by the customer, the material should be kept at $-20^{\circ} \mathrm{C}$ for long-term storage or at $4{ }^{\circ} \mathrm{C}$ for use within eight weeks.

\subsection{Intended use}

This certified reference material (CRM) is intended to support the validation and the harmonisation of PCR-based methods used for detection of the G20210A mutation in the human prothrombin gene. In particular, this CRM is intended to be used as a negative control material in diagnostic tests for the identification and detection of the G20210A mutation in the human prothrombin gene.

Use 0.5 to $5 \mu \mathrm{L}$ of pDNA solution per 20 to $50 \mu \mathrm{L}$ total volume of the PCR reaction (depending on the method and the instrument). Alternatively, the material can be diluted 10-times prior to use; in that case $5 \mu \mathrm{L}$ of the diluted sample should be used, and no stability can be guaranteed anymore.

The plasmid DNA as provided can be kept at $4{ }^{\circ} \mathrm{C}$ for eight weeks or at $-20{ }^{\circ} \mathrm{C}$ for long-term storage. 


\section{References}

[1] Linsinger, T.P.J., Pauwels, J, van der Veen, A.M.H., Schimmel, H., Lamberty, A. (2001) Homogeneity and stability of reference materials. Accred Qual Assur 6, 20-25. [2] van der Veen, A.M.H., Linsinger, T.P.J., Pauwels, J. (2001) Uncertainty calculations in the certification of reference materials. 2. Homogeneity study. Accred Qual Assur 6, 26-30.

[3] van der Veen, A.M.H., Linsinger, T.P.J., Lamberty, A., Pauwels, J. (2001) Uncertainty calculations in the certification of reference materials 3 . Stability study. Accred Qual Assur 6, 257-263.

[4] van der Veen, A.M.H, Linsinger, T.P.J., Schimmel, H., Lamberty, A., Pauwels, J. (2001) Uncertainty calculations in the certification of reference materials 4. Characterisation and certification. Accred Qual Assur 6, 290-294.

[5] http://www.roche-diagnostics.com/ba rmd/rmd products genomics 02.html

[6] Klein, C.L., Marki-Zay, J., Corbisier, P., Gancberg, D., Cooper, S., et al. (2005) Reference materials (RMs) for analysis of the human factor II (prothrombin) gene G20210A mutation. Clin Chem Lab Med 43(8), 862-868.

[7] Handbook of Fluorescent Probes and Research Products, Section 8.3. http://probes.invitrogen.com/handbook, Molecular Probes (2005).

[8] QIAGEN plasmid purification Handbook, 2 $2^{\text {nd }}$ Edition, 2003. http://www1.qiagen.com/literature/handbooks/

[9] Corbisier, P. Validation report Working Instruction RM WI/0090, Fluorescent quantitative determination of ds DNA by PicoGreen, Internal report IRMM (2004).

[10] CEQ 8000 Series - DTCS Chemistry Protocol. http://www.beckman.com/ Literature/BioResearch/390003ac.pdf. Beckman Coulter, Inc. (2003).

[11] Endler, G., Kyrle, P.A., Eichinger, S., Exner, M., Mannhalter, C. (2001) Multiplexed mutagenically separated PCR: simultaneous single-tube detection of the factor V R506Q (G1691A), the prothrombin G20210A, and the methylenetetrahydrofolate reductase A223V (C677T) variants. Clin Chem 47, 333335.

[12] Poort, S.R., Rosendaal, F.R., Reitsma, P.H., Bertina, R.M. (1996) A common genetic variation in the 39-untranslated region of the prothrombin gene is associated with elevated plasma prothrombin levels and increase in venous thrombosis. Blood 88, 3698-3703.

[13] Danneberg, J., Abbes, A.P., Bruggeman, B.J., Engel, H., Gerrits, J., Martens, A. (1998) Reliable genotyping of the G20210A mutation of coagulation factor II (prothrombin). Clin Chem 44, 349-351. 
EUR 22169 EN - DG Joint Research Centre, Institute for Reference Materials and Measurements Certification of a Reference Material consisting of purified plasmid DNA containing a fragment from the human prothrombin gene (wildtype)

Authors: D. Gancberg, J. Marki-Zay, P. Corbisier, C. Klein, H. Schimmel, H. Emons

Luxembourg: Office for Official Publications of the European Communities

$2006-35$ pp. $-21.0 \times 29.7 \mathrm{~cm}$

Scientific and Technical Research series

ISBN 92-79-01633-4

\begin{abstract}
This report describes the production and certification of a reference material consisting of purified plasmid DNA (pDNA) pUC18 containing a 609 base pairs fragment of human Factor II (prothrombin) gene sequence, IRMM/IFCC-490. This certified reference material (CRM) is intended to support the validation and the harmonisation of polymerase chain reaction (PCR)-based methods used for detection of the G20210A mutation in the human prothrombin gene. The homogeneity and short-term stability of the batch was assessed by monitoring the melting temperature $\left(T_{m}\right)$ of the product and the crossing point threshold (Cp) value obtained after PCR amplification using the LightCycler Factor II (prothrombin) G20210A kit and a LightCycler 1.5 instrument. The long-term stability was assessed using the cycle threshold value (Ct) obtained after real-time PCR amplification using specific primers and probe in combination with ABI Prism instruments.

The material is homogeneous and stable at $-20^{\circ} \mathrm{C}$. The sequence of the insert was confirmed by DNA sequence analysis. Each vial contains an indicative volume of $50 \mu \mathrm{L}$ corresponding to approximately $1 \mathrm{ng}$ of pDNA.
\end{abstract}


The mission of the Joint Research Centre is to provide customer-driven scientific and technical support for the conception, development, implementation and monitoring of European Union policies. As a service of the European Commission, the JRC functions as a reference centre of science and technology for the Community. Close to the policy-making process, it serves the common interest of the Member States, while being independent of special interests, whether private or national.

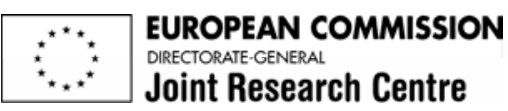


III. 
Certification of a Reference Material consisting of purified plasmid DNA containing a fragment from the human prothrombin gene (G20210A mutant)

\section{Certified Reference Material IRMM/IFCC-491}

D. Gancberg, J. Marki-Zay, P. Corbisier, C. Klein, H. Schimmel, H. Emons

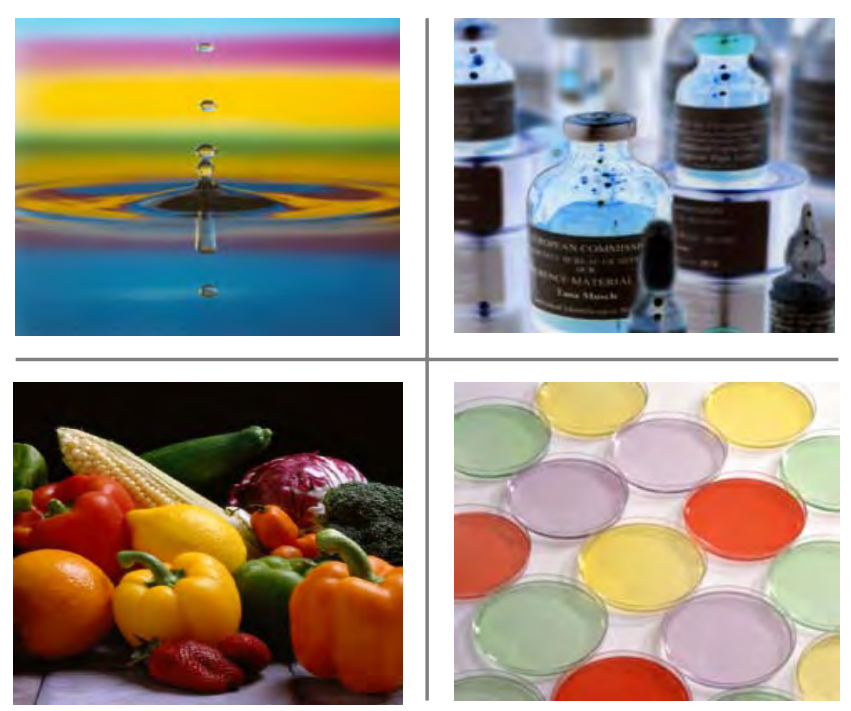


The mission of IRMM is to promote a common and reliable European measurement system in support of EU policies.

\section{European Commission}

Directorate-General Joint Research Centre

Institute for Reference Materials and Measurements

\section{Contact information}

\section{Gancberg}

European Commission

Directorate-General Joint Research Centre

Institute for Reference Materials and Measurements

Retieseweg 111

B-2440 Geel • Belgium

E-mail: david.gancberg@ec.europa.eu

Tel.: +32 (0)14 571911

Fax: +32 (0)14571 548

http://www.irmm.jrc.be

http://www.jrc.cec.eu.int

\section{Legal Notice}

Neither the European Commission nor any person acting on behalf of the Commission is responsible for the use which might be made of the following information.

A great deal of additional information on the European Union is available on the Internet. It can be accessed through the Europa server

http://europa.eu.int

\section{EUR Report 22170 EN}

Luxembourg: Office for Official Publications of the European Communities

ISBN 92-79-01634-2

ISSN 1018-5593

(c) European Communities, 2006

Reproduction is authorised provided the source is acknowledged 


\section{Certification of a Reference Material consisting of purified plasmid DNA containing a fragment from the human prothrombin gene (G20210A mutant)}

\section{IRMM/IFCC-491}

D. Gancberg, J. Marki-Zay, P. Corbusier, H. Schimmel, H. Emons

European Commission

Joint Research Centre

Institute for Reference Materials and Measurements (IRMM)

Retieseweg 111, 2440 Geel, Belgium

\section{C.Klein}

Directorate General Joint Research Centre Institute for Health and Consumer Protection

Via Fermi, 1

IT-21020 Ispra (Varese), Italy 


\section{SUMMARY}

This report describes the production and certification of a reference material consisting of purified plasmid DNA (pDNA) pUC18 containing a 609 base pairs fragment of human Factor II (prothrombin) gene sequence, IRMM/IFCC-491. This certified reference material (CRM) is intended to support the validation and the harmonisation of polymerase chain reaction (PCR)-based methods used for detection of the G20210A mutation in the human prothrombin gene. The homogeneity and short-term stability of the batch was assessed by monitoring the melting temperature $\left(T_{m}\right)$ of the product and the crossing point threshold $(C p)$ value obtained after PCR amplification using the LightCycler Factor II (prothrombin) G20210A kit and a LightCycler 1.5 instrument. The long-term stability was assessed using the cycle threshold value $(\mathrm{Ct})$ obtained after real-time PCR amplification using specific primers and probe in combination with $A B I$ Prism instruments.

The material is homogeneous and stable at $-20{ }^{\circ} \mathrm{C}$. The sequence of the insert (G20210A mutation) was confirmed by DNA sequence analysis. Although a second mutation $(A->G)$ was found at position 20054, its location did not influence the G20210A diagnostic protocol using the Factor II (prothrombin) G20210A kit. Each vial contains an indicative volume of $50 \mu \mathrm{L}$ corresponding to approximately $1 \mathrm{ng}$ of pDNA. 


\section{TABLE OF CONTENTS}

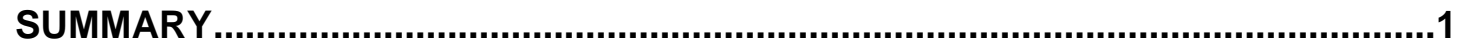

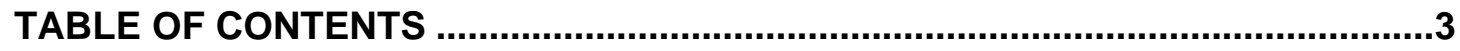

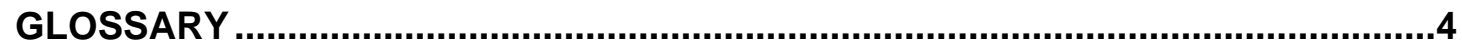

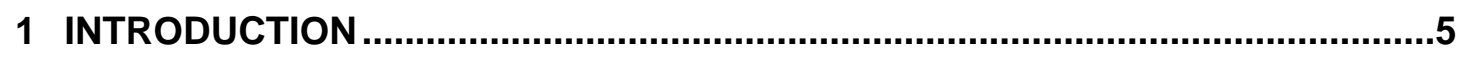

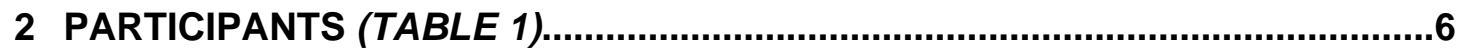

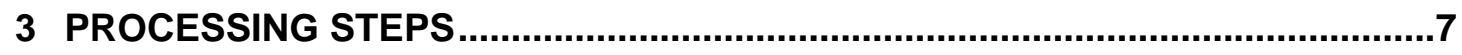

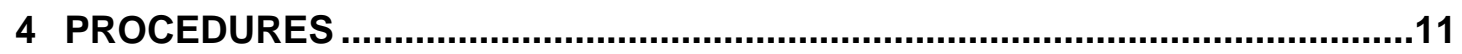

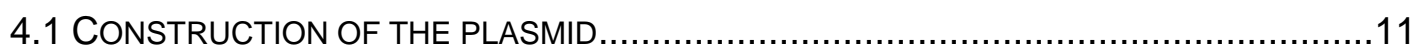

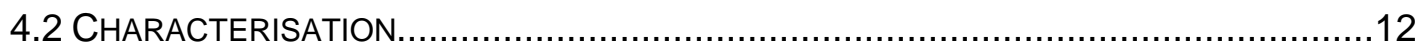

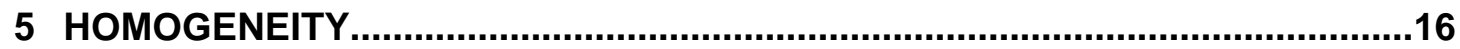

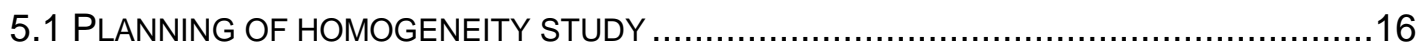

5.2 EVALUATION OF HOMOGENEITY STUDY ..........................................................

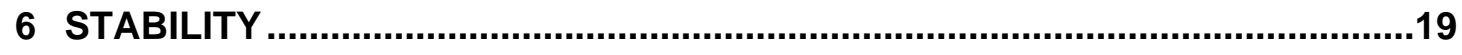

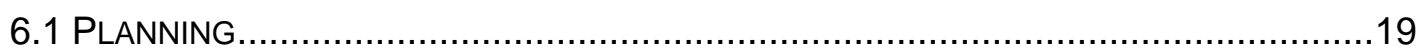

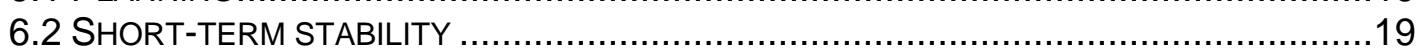

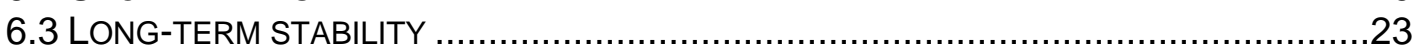

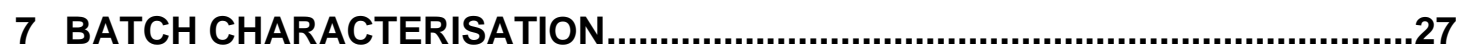

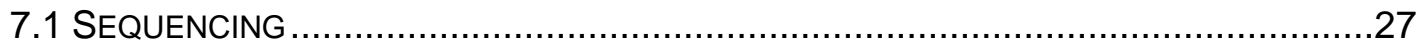

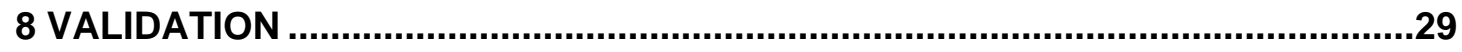

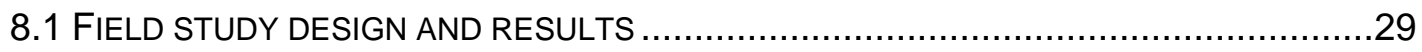

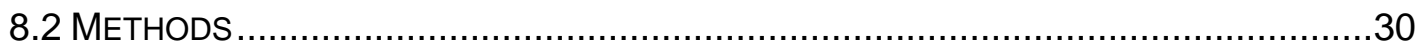

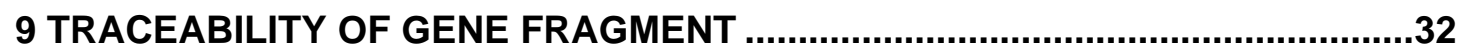

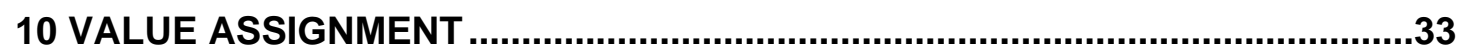

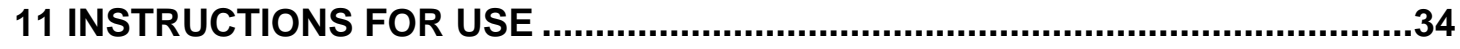

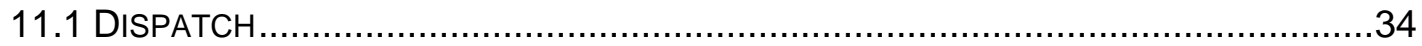

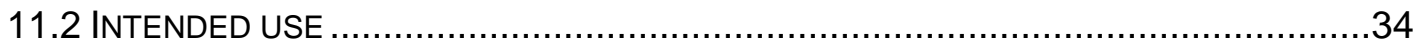

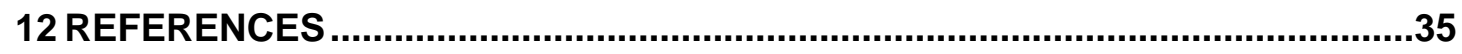




\section{GLOSSARY}

\begin{tabular}{|c|c|}
\hline A & Adenine \\
\hline ANOVA & analysis of variance \\
\hline & slope of the regression line \\
\hline bp & base pairs \\
\hline C & Cytosine \\
\hline Cp & $\begin{array}{l}\text { Crossing point. The } C p \text { value is defined as the crossing point } \\
\text { threshold and defines the threshold above which an amplification } \\
\text { signal can be detected using real-time PCR. }\end{array}$ \\
\hline CRM & Certified Reference Material \\
\hline $\mathrm{Ct}$ & $\begin{array}{l}\text { Cycle threshold. The Ct value is defined as the cycle threshold and } \\
\text { defines the threshold above which an amplification signal can be } \\
\text { detected using real-time PCR. }\end{array}$ \\
\hline DNA & deoxyribo nucleic acid \\
\hline DTCS & Dye Terminator Cycle Sequencing \\
\hline dNTP & deoxy nucleotide triphosphate \\
\hline FAM & 6-carboxy fluorescein \\
\hline FRET & Fluorescence Resonance Energy Transfer \\
\hline & centrifugal force \\
\hline G & Guanine \\
\hline G20210A & $\begin{array}{l}\text { mutation at position } 20210 \text { resulting from the replacement of a } \\
\text { Guanine by an Adenine in the sequence }\end{array}$ \\
\hline IFCC & $\begin{array}{l}\text { International Federation of Clinical Chemistry and Laboratory } \\
\text { Medicine }\end{array}$ \\
\hline IP & Intellectual Property \\
\hline IRMM & Institute for Reference Materials and Measurements \\
\hline LB & Luria Broth \\
\hline$M S_{\text {among }}$ & mean of squares among vials (from ANOVA) \\
\hline$M S_{\text {within }}$ & mean of squares within vials (from ANOVA) \\
\hline$N$ & total number of vials in the homogeneity study \\
\hline $\mathrm{p}$ & probability \\
\hline PCR & polymerase chain reaction \\
\hline pDNA & plasmid deoxyribo nucleic acid \\
\hline $\begin{array}{l}\text { RFLP } \\
\text { rpm }\end{array}$ & $\begin{array}{l}\text { Restriction Fragment Length Polymorphism } \\
\text { revolution per minute }\end{array}$ \\
\hline$s_{\mathrm{bb}}$ & standard deviation between the vials \\
\hline$s$ & absolute standard deviation \\
\hline SOC & glucose-enriched bacterial medium \\
\hline SS & the sum of the squares of the data \\
\hline$t_{\alpha, v}$ & $\begin{array}{l}t \text {-value (two-sided) for a confidence level } \alpha \text { and } v \text { degrees of } \\
\text { freedom (from regression analysis) }\end{array}$ \\
\hline$t$ & time \\
\hline $\mathrm{T}$ & Thymine \\
\hline TAMRA & 6-carboxy-tetra-methyl-rhodamine \\
\hline TBE & $8.9 \mathrm{mmol} / \mathrm{L}$ Tris, $8.9 \mathrm{mmol} / \mathrm{L}$ Boric acid, $0.2 \mathrm{mmol} / \mathrm{L}$ EDTA, $\mathrm{pH} 8.3$ \\
\hline TE & $10 \mathrm{mmol} / \mathrm{L}$ Tris, $1 \mathrm{mmol} / \mathrm{L}$ EDTA, $\mathrm{pH} 8.0$ \\
\hline $\mathrm{T}_{\mathrm{m}}$ & $\begin{array}{l}\text { melting temperature. The melting temperature is the temperature at } \\
\text { which } 50 \% \text { of the oligonucleotides are in double-stranded } \\
\text { conformation and } 50 \% \text { are single stranded. }\end{array}$ \\
\hline$u_{\mathrm{b}}$ * & standard uncertainty of the slope of the regression line \\
\hline$u_{\mathrm{bb}}{ }^{*}$ & $\begin{array}{l}\text { standard uncertainty due to inhomogeneity that can be hidden by } \\
\text { method repeatability. It is used as the minimum uncertainty } \\
\text { contribution from inhomogeneity between vials. } \\
\text { dearees of freedom }\end{array}$ \\
\hline
\end{tabular}




\section{Introduction}

Molecular genetic tests are used in medical diagnostics to identify changes in DNA sequences to diagnose or predict an inherited disorder. These tests should be highly selective. The use of an adequate reference material to validate test equipment and measurement procedures is an important part of any quality control system. Currently, only few CRMs are available for molecular genetic testing.

Thus, in collaboration with the International Federation of Clinical Chemistry and Laboratory Medicine (IFCC), a DNA reference material was produced for the detection of a single point mutation (G20210A) in the gene coding for the human prothrombin (Factor II). This gene mutation has been identified as one of the risk factors in venous thrombosis events.

Currently, several measurement procedures are used among laboratories to detect the G20210A mutation in the prothrombin gene. All of them are based on a PCR amplification followed either by restriction enzyme digestion, hybridisation protocols, single-strand conformation polymorphism analysis, LightCycler PCR analysis, denaturating gradient gel analysis or sequencing.

Therefore, a DNA fragment that spans the area of the human prothrombin gene containing the G20210A mutation has been used to produce the reference material. This specific region can be amplified with all primer combinations described in the literature for prothrombin mutation analysis.

Besides the mutated DNA fragment, the wildtype DNA fragment was produced as well. Both fragments were cloned separately into the pUC18 plasmid. The concentration per vial was adjusted to $1 \mathrm{ng}$ per $50 \mu \mathrm{L}$.

For the present CRM IRMM/IFCC-491, a batch of 1500 vials of purified pDNA containing a cloned $609 \mathrm{bp}$ fragment of the human prothrombin gene with a mutation G->A at position 20210 was produced. The certification of IRMM/IFCC-491 was performed according to IRMM RM Unit procedures applying the ISO Guide 34 quality management system [1-4]. Stability measurements were performed at three different temperatures $\left(-20^{\circ} \mathrm{C}, 4^{\circ} \mathrm{C}, 18^{\circ} \mathrm{C}\right)$. Sequence identity in the production batch before dilution was assessed by DNA sequence analysis of the fragment inserted into the pUC18 vector.

The produced batch was characterised for homogeneity.

Samples in short-term stability measurements were analysed by melting curve experiment using PCR and the commercial LightCycler kit for Factor II (prothrombin) G20210A mutation detection [5]. Samples in long-term stability measurements were analysed by real-time PCR experiments using specific primers and probe. 


\section{Participants (Table 1)}

The following laboratories with expertise in genetic testing were involved in the field study:

Table 1: List of participants for the characterisation of the material

\begin{tabular}{|l|l|l|}
\hline Halbmayer, W. & $\begin{array}{l}\text { Institut für Medizinische und Chemische } \\
\text { Labordiagnostik mit Thromboseambulanz / } \\
\text { Krankenhaus der Stadt Wien Lainz }\end{array}$ & AT-1130 Wien \\
\hline Zadro, R. & $\begin{array}{l}\text { Clinical Institute of Laboratory Diagnosis / Clinical } \\
\text { Hospital Center Zagreb University School of } \\
\text { Medicine }\end{array}$ & HR-10000 Zagreb \\
\hline Melegh, B. & $\begin{array}{l}\text { University of Pecs, Department of Medical } \\
\text { Genetics and Child Development }\end{array}$ & HU-7624 Pecs \\
\hline Gemmati, D. & $\begin{array}{l}\text { Ctr. Study Haemostasis and Thrombosis, } \\
\text { University of Ferrara }\end{array}$ & IT-44100 Ferrara \\
\hline Spannagl, M. & $\begin{array}{l}\text { Ludwig-Maximilians-Universität, Klinikum } \\
\text { Innenstadt, Hämostaseologische Ambulanz }\end{array}$ & DE-80336 Munich \\
\hline Kitchen, S. & $\begin{array}{l}\text { Dept. of Coagulation / Sheffield Haemophilia and } \\
\text { Thrombosis Centre / Royal Hallamshire Hospital }\end{array}$ & GB- S102 JF Sheffield \\
\hline Mannhalter, C. & $\begin{array}{l}\text { Klinisches Institut für Medizinische und } \\
\text { Chemische Labordiagnostik (KIMCL) }\end{array}$ & AT-1090 Wien \\
\hline
\end{tabular}




\section{Processing steps}

Prof. C. Mannhalter, Clinical Institute of Medical and Chemical Laboratory Diagnostics, General Hospital and Medical University Vienna (Vienna, Austria) isolated and purified a $609 \mathrm{bp}$ fragment of the prothrombin gene from a patient containing the G->A mutation at position 20210, and cloned it into vector pCR2.1 (TA Cloning ${ }^{\circledR}$ kit, Invitrogen). A solution with approximately $10^{6}$ copies of this plasmid in $100 \mu \mathrm{L}$ TE buffer $\mathrm{pH} 8.0$ was delivered frozen to IRMM. The production and certification of IRMM/IFCC-491 was performed at IRMM.

The processing steps for the production of IRMM/IFCC-491 are described by Klein et al. [6] and in the procedures of Chapter 4. As the pCR2.1 vector is patented, it was necessary to subclone the fragment in a non-IP protected vector. Briefly, the $609 \mathrm{bp}$ fragment was subcloned into the pUC18 cloning vector (Figure 1). Two primers, designed to obtain the Factor II insert of the pCR2.1 plasmid flanked by a EcoRI restriction site were used to amplify a fragment that could subsequently be inserted into pUC18, via the unique EcoRI site.

Qualitative PCR was applied (see Chapter 4), $5 \mu \mathrm{L}$ of the plasmid (at $10^{4}$ copies $/ \mu \mathrm{L}$ ) were amplified using primers P-0342 and P-0343 (Table 2).

Table 2: Primers used for cloning in EcoRI site

\begin{tabular}{|l|l|l|}
\hline & $\begin{array}{l}\text { IRMM Primer Code } \\
\text { number }\end{array}$ & Primer sequence \\
\hline Forward & P-0342 & 5'-CGAGCTCGGATCCACTAGTAA-3' \\
\hline Reverse & P-0343 & 5'-GCCAGTGTGATGGATATCTGC-3' \\
\hline
\end{tabular}

The amplification products were controlled by agarose gel electrophoresis for their correct molar mass (length in bp) by comparison to a DNA molecular mass ladder and purified using the Qiagen PCR fragment purification kit. Subsequently, PCR fragments and pUC18 plasmid were digested with EcoRI. After the digestion, the restriction enzyme was heat-inactivated for $20 \mathrm{~min}$ at $65{ }^{\circ} \mathrm{C}$ and the linearised vector pUC18 was dephosphorylated for 30 min at $37^{\circ} \mathrm{C}$ using the shrimp alkaline phosphatase in order to prevent recircularisation of the plasmid. The dephosphorylated vector and the insert were then ligated overnight at $14{ }^{\circ} \mathrm{C}$. The resulting new plasmid containing the 609 bp wildtype fragment of Factor II with a point mutation at position 20210 was coded pIRMM-0002. 


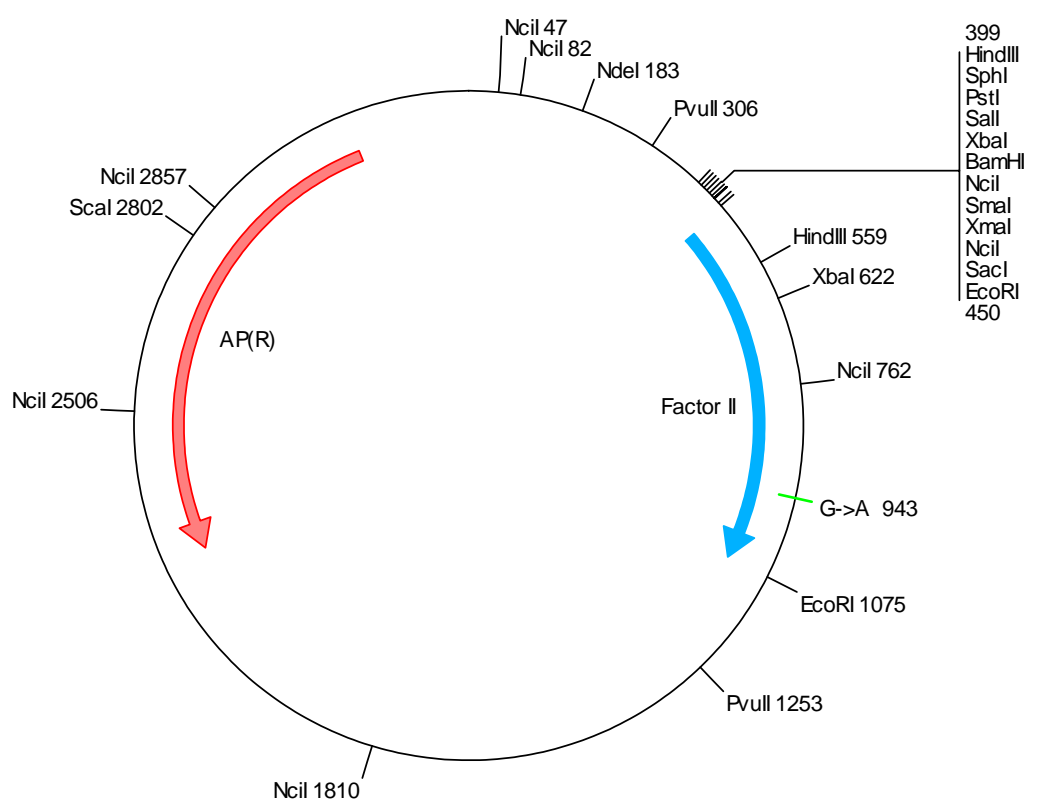

Figure 1: Map of plasmid pIRMM-0002 (3311 bp, G20210A point mutation). The factor II mutation G->A is located at position 943

The resulting plasmid pIRMM-0002 was transformed into E. coli TOP10.

Ten colonies of bacteria were tested for the presence of the insert. Therefore, $a$ sample of each colony was introduced as template in a qualitative PCR reaction mix using the specific Factor II primers (P-0308 and P-0309, Table 3). The following PCR cycling programme was used in the GeneAmp 9700 System: melting pDNA at $95{ }^{\circ} \mathrm{C}$ for $10 \mathrm{~min}$; 40 cycles of denaturation at $95^{\circ} \mathrm{C}$ for $1 \mathrm{~min}$, annealing at $58^{\circ} \mathrm{C}$ for $1 \mathrm{~min}$, extension at $72{ }^{\circ} \mathrm{C}$ for $1 \mathrm{~min}$; incubation at $72{ }^{\circ} \mathrm{C}$ for $10 \mathrm{~min}$; hold at $4{ }^{\circ} \mathrm{C}$.

Table 3: Primers used for Factor II sequence amplification

\begin{tabular}{|l|l|l|}
\hline & $\begin{array}{l}\text { IRMM Primer Code } \\
\text { number }\end{array}$ & Primer sequence \\
\hline Forward & P-0308 & 5'-GCACAGACGGCTGTTCTCTT-3' \\
\hline Reverse & P-0309 & 5'-CCCGAGTGCTCGGACTACCA-3' \\
\hline
\end{tabular}

The analysis of the different colonies revealed that all colonies contained the plasmids. Clone "number 4" was selected and a HindIII and EcoRI digest was carried out in order to assess the orientation of the insert (Figure 2). 


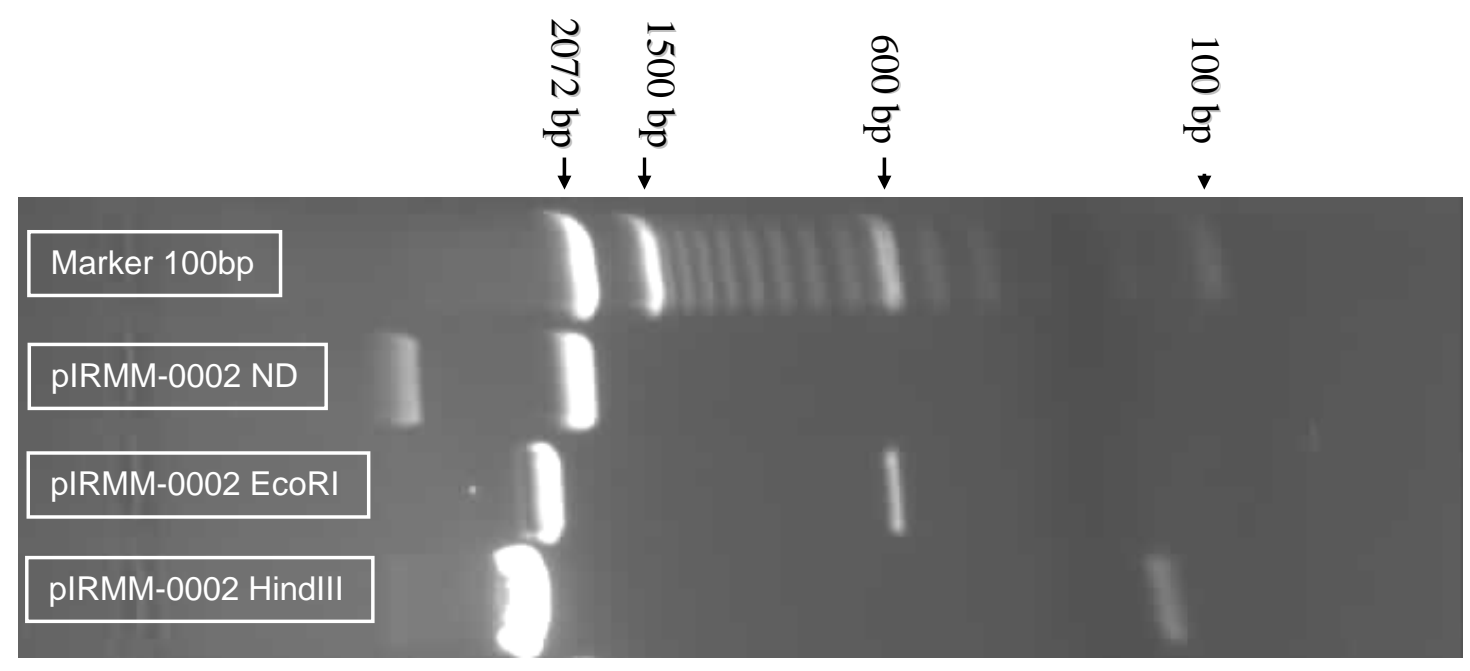

Figure 2. Agarose gel (mass fraction = $1 \%$ ) electrophoresis of pIRMM-0002. $10 \mu \mathrm{L}$ of pDNA digested with EcoRI (lane 3), with HindIII (lane 4), or not digested (ND, lane 2) were mixed with $10 \mu \mathrm{L}$ DNase free water and $2.2 \mu \mathrm{L}$ of 10-times gel loading buffer. The agarose gel in TBE buffer was stained with $0.5 \mu \mathrm{g} / \mathrm{mL}$ Ethidium Bromide; the electrophoresis was performed using the following conditions: $100 \mathrm{~V}, 45$ min; marker: 100 bp Ready-Load DNA (Invitrogen, Merelbeke, Belgium, lane 1).

The undigested plasmid exists under the supercoiled (heavier) and linear forms. The EcoRI digestion generated fragment sizes of $625 \mathrm{bp}$ and $2686 \mathrm{bp}$. If the fragment is in the desired orientation (see Figure 1), fragments size of $160 \mathrm{bp}$ and $3151 \mathrm{bp}$ should be obtained after HindIII digestion.

The size of the fragments generated after digestion indicated that the insert was in the desired orientation. Two clones containing the sequence of plasmid pIRMM-0002 were kept as glycerol stock at $-70{ }^{\circ} \mathrm{C}$ and coded C-0002 (clone 4) and C-0004 (clone 1).

The plasmids from strain C-0001 were purified on large scale using the QIAgen plasmid purification Gigakit and the concentration of the plasmids was measured by double-stranded DNA PicoGreen quantitation method [7]. The average pDNA stock concentration and standard deviation was $1823 \pm 29 \mu \mathrm{g} / \mathrm{mL}$.

\section{Production}

The pDNA production follows the QIAgen Gigakit plasmid purification protocol.

1. A bacterial culture ( $10 \mathrm{~mL}$ of $\mathrm{LB}$ medium $+100 \mu \mathrm{g} / \mathrm{mL}$ ampicillin) was inoculated with $100 \mu \mathrm{L}$ of strain C-0002 from glycerol stock containing the pIRMM-0002 plasmid and grown at $37^{\circ} \mathrm{C}$ for $8 \mathrm{~h}$ with orbital shaking at $300 \mathrm{rpm}$. At the end of the day a LB agar plate containing $100 \mu \mathrm{g} / \mathrm{mL}$ ampicillin was plated out and placed at $37{ }^{\circ} \mathrm{C}$ overnight (in order to be able to pick up a single colony the next day). 
2. A single colony was cultivated as primary culture at $37^{\circ} \mathrm{C}$ for $8 \mathrm{~h}$ in $10 \mathrm{~mL} \mathrm{LB}$ medium $+100 \mu \mathrm{g} / \mathrm{mL}$ ampicillin with orbital shaking at $300 \mathrm{rpm}$. Four flasks of 500 $\mathrm{mL}$ LB medium $+100 \mu \mathrm{g} / \mathrm{mL}$ ampicillin were then incubated overnight with $2.5 \mathrm{~mL}$ of a primary culture and grown at $37^{\circ} \mathrm{C}$ with orbital shaking at $275 \mathrm{rpm}$.

3. Bacterial cells were centrifugated for $15 \mathrm{~min}$ at $6000 \mathrm{~g}$ at $4{ }^{\circ} \mathrm{C}$ using a Avanti J-20 Beckman centrifuge. Plasmid DNA was purified according to the procedure from QIAgen plasmid purification (second edition, August 2003, [8]).

4. The plasmid DNA pellet obtained at the end of the purification was resuspended in $5 \mathrm{~mL}$ TE pH 8.0 overnight, aliquoted in $5 \times 1 \mathrm{~mL}$ and stored at $-20^{\circ} \mathrm{C}$.

5 . The concentration of double-stranded pDNA was determined by fluorescence of PicoGreen [7] using lambda phage DNA as external standard. The integrity of pDNA was verified visibly by agarose gel electrophoresis.

6. The plasmid stock solution was diluted in $\mathrm{TE} \mathrm{pH} 8.0$ to a final concentration of $0.02 \mathrm{ng} \mathrm{pDNA} / \mu \mathrm{L}$ or $1 \mathrm{ng} / 50 \mu \mathrm{L}$.

7. One thousand five hundred polypropylene vials (sterile, $1.5 \mathrm{~mL}$ volume) were filled manually with $50 \mu \mathrm{L}$ of pDNA solution (mass concentration $0.02 \mathrm{ng} / \mu \mathrm{L} \mathrm{TE}$ buffer, $\mathrm{pH}$ 8.0).

8. The vials were closed with screw caps in a laminar flowhood and labelled.

9. Homogeneity and stability studies as well as batch characterisation were carried out. 


\section{Procedures}

\subsection{Construction of the plasmid}

\section{Enzymatic Digestion}

The digestion reaction was composed of $10 \mu \mathrm{L}$ of plasmid, $3 \mu \mathrm{L}$ buffer 10-times concentrated (SURE Cut buffer H for EcoRI, SURE Cut buffer B for HindIII, Roche, Vilvoorde, Belgium), $16 \mu \mathrm{L}$ Promega nuclease-free water and $1 \mu \mathrm{L}$ enzyme (either HindIII or EcoRI, Promega, Leiden, The Netherlands).

\section{Dephosphorylation}

The reaction mix for dephosphorylation was prepared by mixing $10 \mu \mathrm{L}$ plasmid linearized (by EcoRI), $2 \mu \mathrm{L}$ buffer 10-times concentrated (Roche, Vilvoorde,Belgium), $8 \mu \mathrm{L}$ enzyme shrimp alkaline phosphatase (SAP, Roche,Vilvoorde, Belgium); the SAP was further inactivated at $65^{\circ} \mathrm{C}$ for $15 \mathrm{~min}$.

\section{Ligation}

The reaction mix was prepared as follows: $5 \mu \mathrm{L}$ Promega nuclease-free water, $1 \mu \mathrm{L}$ of 10-times concentrated buffer (Roche, Vilvoorde, Belgium), $1.5 \mu \mathrm{L}$ vector and $1 \mu \mathrm{L}$ insert:, $1 \mu \mathrm{L}$ of T4 DNA ligase (Westburg, Leusden, The Netherlands).

\section{Transformation}

The TOP10 competent E.coli (Invitrogen, Merelbeke, Belgium) were thawed on ice (one shot aliquots) and $2 \mu \mathrm{L}$ of the ligation reaction was added and mixed gently by tapping. After incubation $30 \mathrm{~min}$ on ice, a heat shock was performed at $42{ }^{\circ} \mathrm{C}$ for exactly $30 \mathrm{~s}$. The tube was then placed on ice for $2 \mathrm{~min}$, and $250 \mu \mathrm{L}$ of SOC medium was added to the tube. The tube was then incubated at $37^{\circ} \mathrm{C}$ for exactly $1 \mathrm{~h}$ with shaking at $225 \mathrm{rpm}$. The tube was placed on ice, then $50 \mu \mathrm{L}$ or $200 \mu \mathrm{L}$ of the mixture was plated overnight at $37^{\circ} \mathrm{C}$ on LB Agar medium containing antibiotic $(100 \mu \mathrm{g} / \mathrm{mL}$ ampicillin or kanamycin).

\section{Bacterial media}

LB Broth (Fluka, Bornem, Belgium): dissolve $20 \mathrm{~g} / \mathrm{L}$ (Tryptone $10 \mathrm{~g} / \mathrm{L}$, Yeast extract 5 $\mathrm{g} / \mathrm{L}$, Sodium Chloride $5 \mathrm{~g} / \mathrm{L}$ ) in water, adjust to $\mathrm{pH} 7.2$, sterilize for $15 \mathrm{~min}$ at $121^{\circ} \mathrm{C}$. LB Agar (Fluka, Bornem, Belgium): dissolve $30 \mathrm{~g} / \mathrm{L}$ (Tryptone $10 \mathrm{~g} / \mathrm{L}$, Yeast extract 5 $\mathrm{g} / \mathrm{L}$, Sodium Chloride $5 \mathrm{~g} / \mathrm{L}$, Agar $10 \mathrm{~g} / \mathrm{L}$ ) in water, adjust to $\mathrm{pH} 7.2$, sterilize for 15 $\min$ at $121^{\circ} \mathrm{C}$.

SOC medium (Fluka, Bornem, Belgium): ready-to-use medium, sterile. Contains Tryptone $20 \mathrm{~g} / \mathrm{L}$, Yeast extract $5 \mathrm{~g} / \mathrm{L}$, Glucose $20 \mathrm{mmol} / \mathrm{L}$. 


\section{Ampicillin}

$100 \mathrm{mg} / \mathrm{mL}$ Ampicillin sodium salt (Sigma, Belgium, cat\#A0166-5G) was dissolved in water and sterilized by $0.22 \mu \mathrm{m}$ filtering. This solution stock was aliquoted and diluted 1000 -times ( $\mathrm{vol} / \mathrm{vol})$ to reach the working concentration of $100 \mu \mathrm{g} / \mathrm{mL}$.

\section{Agarose gel electrophoresis}

To $10.0 \mu \mathrm{L}$ sample, of either pDNA or PCR product, $2.2 \mu \mathrm{L}$ of 10 -times concentrated gel loading buffer (Invitrogen, Merelbeke, Belgium) was mixed as well as $10 \mu \mathrm{L}$ of Promega nuclease-free water. $20 \mu \mathrm{L}$ of this sample was loaded on a $1 \%$ (mass fraction) agarose gel in TBE buffer with $0.5 \mu \mathrm{g} / \mathrm{mL}$ Ethidium Bromide (Sigma, St. Louis, USA). Electrophoresis was performed at $100 \mathrm{~V}$ for 45 min using a Mini-Sub Cell GT Electrophoresis system and a Power Pac 3000 (Bio-Rad, Nazareth, Belgium). Gels were photographed on a GeneScan Gel Documentation System (Syngene, Cambridge, United Kingdom).

\subsection{Characterisation}

\section{Minimum sample volume}

The minimum sample volume was $5 \mu \mathrm{L}$ as recommended in the protocol of the Factor II (prothrombin) G20210A kit used in combination with the LightCycler. Dilution experiments during the short-term stability study gave an indication of the limit of detection.

\section{Quantification of pDNA}

Quantification of double-stranded pDNA in stock solution was performed with PicoGreen reagent in a microtiter plate using fluorimetry (excitation at $480 \mathrm{~nm}$ and emission at $525 \mathrm{~nm}$ ) [7]. A standard DNA calibration curve was prepared with lambda phage DNA at mass concentrations ranging from 0 to $1 \mathrm{ng} / \mu \mathrm{L}$. The test was prepared in a microtiter plate, and fluorescence was detected in a FLUOstar Galaxy plate reader (BMG LABTECH GmbH, Offenburg, Germany) [9].

\section{Qualitative PCR}

Each reaction mixture for PCR reactions ( $50 \mu \mathrm{L}$ total volume) contained PCR buffer, $1.5 \mu \mathrm{mmol} / \mathrm{L} \quad \mathrm{MgCl}_{2}, 200 \mu \mathrm{mol} / \mathrm{L}$ dNTP, $0.1 \mu \mathrm{mol} / \mathrm{L}$ forward primer, $0.1 \mu \mathrm{mol} / \mathrm{L}$ reverse primer, 0.5 unit Platinum ${ }^{\circledR}$ Taq DNA polymerase and $5 \mu \mathrm{L}$ pDNA template. PCR primers (Applied Biosystems, Lennik, Belgium) used are summarized in Tables 2 and 3. All other reaction components for PCR reactions were provided by Invitrogen (Merelbeke, Belgium). PCR reactions were performed using the following 
cycling programme: melting pDNA at $95{ }^{\circ} \mathrm{C}$ for $10 \mathrm{~min} ; 40$ cycles of denaturation 95 ${ }^{\circ} \mathrm{C}$ for $1 \mathrm{~min}$, annealing $58^{\circ} \mathrm{C}$ for $1 \mathrm{~min}$, extension $72{ }^{\circ} \mathrm{C}$ for $1 \mathrm{~min}$; incubation at 72 ${ }^{\circ} \mathrm{C}$ for $10 \mathrm{~min}$; hold at $4{ }^{\circ} \mathrm{C}$. PCR products were visualised by agarose gel electrophoresis.

\section{Quantitative real-time PCR}

The principle of the quantitative real-time PCR is the use of a specific fluorescent probe in addition to the primers. The fluorescence intensity is proportional to the number of target DNA sequences. This fluorescence is recorded on-line during the amplification process. The TaqMan ${ }^{\circledR}$ probe contains a reporter dye (FAM) at its 5' end and a quencher (TAMRA) dye at its 3 ' end. Accumulation of PCR products is detected directly by monitoring the increase in fluorescence of the reporter dye. The number of cycles which are required to pass a fluorescence threshold (Ct-value) correlates with the amount of target DNA in the original sample.

We used the ABI Prism 7700 and ABI Prism 7900HT device (Applied Biosystems, Lennik, Belgium).

Each reaction mixture for real-time PCR reactions (25 $\mu \mathrm{L}$ total volume) contained $12.5 \mu \mathrm{L}$ of 2-times concentrated Universal PCR Master Mix (Applied Biosystems, Lennik, Belgium), $2.5 \mu \mathrm{L}$ of forward primer (9 $\mu \mathrm{mol} / \mathrm{L}), 2.5 \mu \mathrm{L}$ of reverse primer (9 $\mu \mathrm{mol} / \mathrm{L}), 2.5 \mu \mathrm{L}$ of probe $(2 \mu \mathrm{mol} / \mathrm{L})$ and $5 \mu \mathrm{L}$ pDNA template. The sequences of PCR primers and probe (Applied Biosystems, Lennik, Belgium) used are summarized in Table 4. PCR reactions were performed using the following cycling programme: 2 $\min$ at $50{ }^{\circ} \mathrm{C}, 10 \mathrm{~min}$ at $95^{\circ} \mathrm{C},\left(15 \mathrm{~s}\right.$ at $95^{\circ} \mathrm{C}, 1 \mathrm{~min}$ at $\left.60^{\circ} \mathrm{C}\right)$ for $30 \mathrm{cycles}$. Ct values were obtained using the SDS 2.2.2 software on ABI Prism $7900 \mathrm{HT}$ and SDS 1.9.1 on ABI Prism 7700 instrument.

Each sample was analysed in triplicate. Triplicates were randomized on the 96-wells plates, and an internal control (pDNA at $0.02 \mathrm{ng} / \mu \mathrm{L}$ ) was displayed on each plate to allow comparison of data on the different instruments.

Table 4. Primers and probe used for the quantitative PCR

\begin{tabular}{|l|l|l|}
\hline & $\begin{array}{l}\text { IRMM Primer } \\
\text { Code number }\end{array}$ & Sequence \\
\hline Forward & P-0564 & 5'-GCTCACCGGCTCCAGATTT -3' \\
\hline Reverse & P-0565 & 5'- TTCTGCGCTCGGCCC-3' \\
\hline Probe & P-0566 & FAM-5'-TCAGCAATAAACCAGCCAGCCGGA-3'-TAMRA \\
\hline
\end{tabular}




\section{Genotyping using the LightCycler technology}

The LightCycler Factor II (prothrombin) G20210A kit (Roche, cat \#036 10195001, [5]) provides primers and hybridisation probes for sequence-specific detection and includes a control template heterozygous for the G20210A mutation for reliable interpretation, as well as a negative control consisting of DNA-free water.

Principle: combining hybridisation probe detection with subsequent melting curve analysis makes applications such as rapid genotyping and mutation analysis possible (example in Figure $3 \mathrm{~A}$ and $3 \mathrm{~B}$ ). Indeed, a single mismatch between the hybridisation probe and its target sequence, caused by a single point mutation, changes the melting temperature of the bound probe. Therefore, the recording of the melting temperature $\left(T_{m}\right)$ in the plot of the negative first derivative of fluorescence intensity versus temperature (-dF/dT) indicates the genotype of the PCR product (Figure 3B).

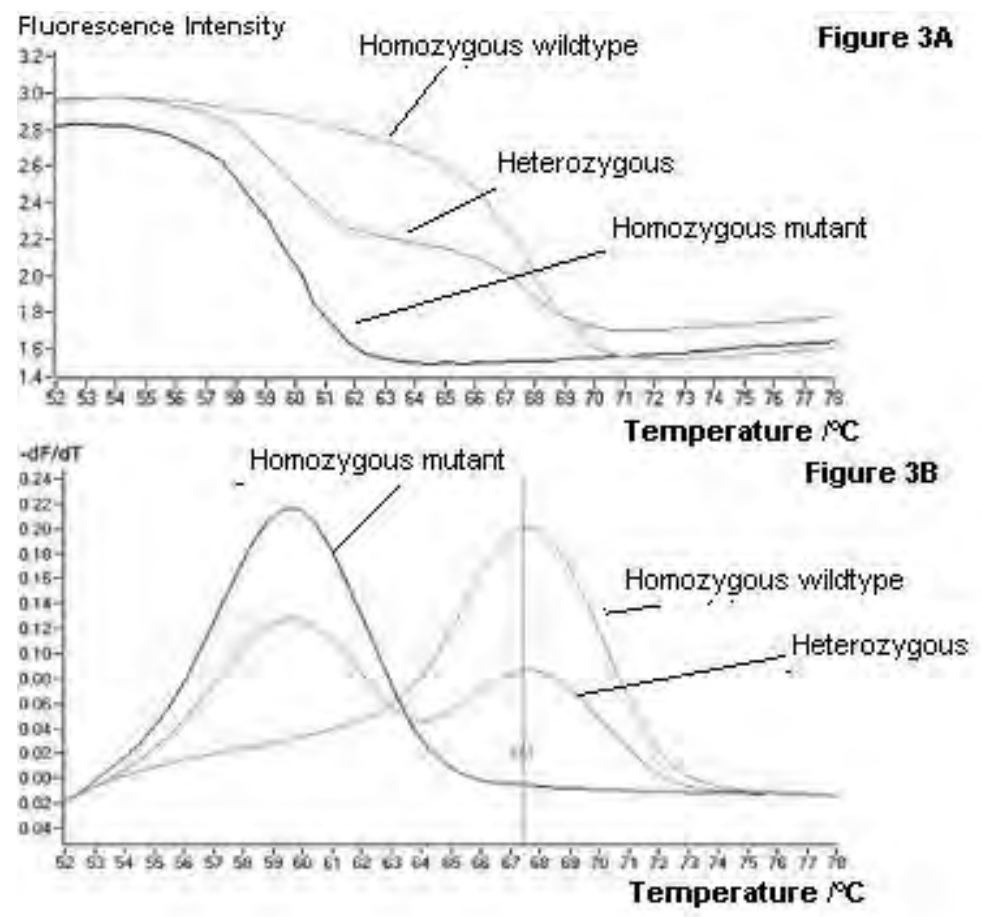

Figure 3: Example of analysis and interpretation of data using the LightCycler technology and the melting curve analysis

The $T_{m}$ does not only depend on the length and $G+C$ content, but also depends on the degree of homology between the mutation probe and the template DNA. In the LightCycler Factor II (prothrombin) G20210A kit, the G20210A mutant genotype and the fluorescein-labeled hybridization probe form a mismatch resulting in a $T_{m}$ of about $49{ }^{\circ} \mathrm{C}$. The kit manual stipulates that the $\mathrm{T}_{\mathrm{m}}$ values may vary $\pm 2.5{ }^{\circ} \mathrm{C}$ between different experiments.

Quantitative data on the DNA used as template in the PCR reaction is given by the Crossing point $(\mathrm{Cp})$ threshold obtained "by automatic call" in the program. The Cp 
value is defined as the crossing point threshold and defines the threshold above which an amplification signal can be detected.

\section{DNA sequencing}

Between 100 and 200 ng of pDNA were used in Dye Terminator Cycle Sequencing (DTCS) reaction, according to the DTCS Chemistry Protocol [10] and using the primers P-0289, P-0419, P-0579 and P-0580 (Table 5). The following thermal cycling programme was used: 30 cycles of $96{ }^{\circ} \mathrm{C}$ for $20 \mathrm{~s}, 50{ }^{\circ} \mathrm{C}$ for $20 \mathrm{~s}, 60{ }^{\circ} \mathrm{C}$ for $4 \mathrm{~min}$; and hold at $4{ }^{\circ} \mathrm{C}$. Samples were analysed on a CEQ ${ }^{\text {TM }} 8000$ Genetic Analysis System (Beckman Coulter, Inc., Fullerton, CA, USA), using the following method: denaturation $120 \mathrm{~s}$ at $90{ }^{\circ} \mathrm{C}$, injection $15 \mathrm{~s}$ at $2.0 \mathrm{kV}$, separation $85.0 \mathrm{~min}$ at $4.2 \mathrm{kV}$.

Table 5: Primers used for Factor II sequencing

\begin{tabular}{|l|l|l|}
\hline & $\begin{array}{l}\text { IRMM Primer } \\
\text { Code number }\end{array}$ & Sequence \\
\hline Forward (M13-F) & P-0419 & 5'- GTA AAA CGA CGG CCA G-3' \\
\hline Reverse (M13-R) & P-0289 & 5'- CAG GAA ACA GCT ATG AC-3' \\
\hline Forward (Factor II-F) & P-0579 & 5'- TAC TGA TGT GAC CTT GAA CT-3' \\
\hline Reverse (Factor II-R) & P-0580 & 5'- CTT CTG TAT CCA CTT CTT CA-3' \\
\hline
\end{tabular}




\section{Homogeneity}

\subsection{Planning of homogeneity study}

1500 vials were produced. The minimum required number of vials used in the homogeneity study $(N)$ is calculated as ${ }^{3} \sqrt{1500}=12$ [1-2]. The homogeneity study was performed using data from samples kept at $-70{ }^{\circ} \mathrm{C}(N=6)$ and at $-20{ }^{\circ} \mathrm{C}(\mathrm{N}=18)$ in the short-term stability study. Two replicates of $5 \mu \mathrm{L}$ of IRMM/IFCC-491 were analysed using the LightCycler prothrombin G20210A mutation detection kit and the Cp values were assessed.

The average of the melting temperature of the product was recorded additionally as it confirmed the identity of the sequence (using defined primers and probes).

\subsection{Evaluation of homogeneity study}

The results of the Crossing point threshold $(C p)$ and average $T_{m}$ measurements are summarized in Table 6. The positive control was a fragment of human DNA, heterozygous for the mutation, and the negative control was DNA-free water (both are included in the kit). 
Table 6. Homogeneity of the $\mathrm{Cp}$ and $\mathrm{T}_{\mathrm{m}}$ values of IRMM/IFCC-491. Vials were stored at $-70^{\circ} \mathrm{C}$ or at $-20^{\circ} \mathrm{C}$. Suspicious data are in bold.

\begin{tabular}{|c|c|c|c|c|c|c|c|}
\hline \multicolumn{8}{|c|}{ Homogeneity of $\mathrm{Cp}$ and $\mathrm{T}_{\mathrm{m}}$ values after storage at $-70^{\circ} \mathrm{C}$ or $-20^{\circ} \mathrm{C}$ for 2,4 or 8 weeks } \\
\hline $\begin{array}{c}\text { Vial } \\
\text { Number }\end{array}$ & $\begin{array}{c}\text { Storage } \\
\text { time at -70 } \\
{ }^{\circ} \mathrm{C} \text { (weeks) }\end{array}$ & $\begin{array}{c}\text { Replicate } \\
1 \\
\end{array}$ & $\begin{array}{c}\text { Replicate } \\
2\end{array}$ & $\begin{array}{c}\text { Average } \\
\mathrm{Cp}\end{array}$ & $s$ Cp & $\begin{array}{c}\text { Average } \\
\mathbf{T}_{\mathrm{m}}\end{array}$ & $s T_{m}$ \\
\hline 9 & 0 & 15.34 & 15.32 & 15.33 & 0.01 & 49.96 & 0.08 \\
\hline 232 & 0 & 15.56 & 15.13 & 15.35 & 0.30 & 50.01 & 0.11 \\
\hline 469 & 0 & 15.89 & 15.51 & 15.70 & 0.27 & 49.94 & 0.09 \\
\hline 706 & 0 & 15.31 & 15.17 & 15.24 & 0.10 & 49.88 & 0.12 \\
\hline 945 & 0 & 15.43 & 15.42 & 15.43 & 0.01 & 49.88 & 0.06 \\
\hline 1166 & 0 & 15.56 & 15.33 & 15.45 & 0.16 & 49.92 & 0.08 \\
\hline $\begin{array}{c}\text { Vial } \\
\text { Number }\end{array}$ & $\begin{array}{c}\text { Storage } \\
\text { time at -20 } \\
{ }^{\circ} \mathrm{C} \text { (weeks) }\end{array}$ & $\begin{array}{c}\text { Replicate } \\
1\end{array}$ & $\begin{array}{c}\text { Replicate } \\
2 \\
\end{array}$ & $\begin{array}{c}\text { Average } \\
\mathrm{Cp}\end{array}$ & $s \mathrm{Cp}$ & $\begin{array}{c}\text { Average } \\
\mathbf{T}_{\mathrm{m}}\end{array}$ & $s T_{m}$ \\
\hline 81 & 2 & 15.21 & 15.15 & 15.18 & 0.04 & 49.96 & 0.05 \\
\hline 310 & 2 & 15.51 & 15.57 & 15.54 & 0.04 & 49.94 & 0.06 \\
\hline 550 & 2 & 15.28 & 15.15 & 15.22 & 0.09 & 49.96 & 0.11 \\
\hline 775 & 2 & 15.37 & 15.22 & 15.30 & 0.11 & 49.95 & 0.02 \\
\hline 1024 & 2 & 15.64 & 15.17 & 15.41 & 0.33 & 49.90 & 0.18 \\
\hline 1250 & 2 & 15.20 & 15.01 & 15.11 & 0.13 & 50.15 & 0.41 \\
\hline 85 & 4 & 15.41 & 15.13 & 15.27 & 0.20 & 49.94 & 0.01 \\
\hline 329 & 4 & 13.38 & 13.99 & 13.69 & 0.43 & 50.05 & 0.05 \\
\hline 560 & 4 & 15.02 & 15.43 & 15.23 & 0.29 & 50.01 & 0.11 \\
\hline 796 & 4 & 15.16 & 14.89 & 15.03 & 0.19 & 49.97 & 0.06 \\
\hline 1029 & 4 & 15.18 & 14.69 & 14.94 & 0.35 & 49.87 & 0.08 \\
\hline 1268 & 4 & 14.69 & 15.10 & 14.90 & 0.29 & 49.92 & 0.01 \\
\hline 91 & 8 & 15.10 & 15.02 & 15.06 & 0.06 & 50.10 & 0.23 \\
\hline 337 & 8 & 14.94 & 14.79 & 14.87 & 0.11 & 50.11 & 0.24 \\
\hline 584 & 8 & 14.97 & 15.07 & 15.02 & 0.07 & 50.06 & 0.16 \\
\hline 808 & 8 & 15.03 & 15.07 & 15.05 & 0.03 & 50.03 & 0.23 \\
\hline 1050 & 8 & 15.26 & 15.08 & 15.17 & 0.13 & 50.05 & 0.24 \\
\hline 1270 & 8 & 15.11 & 15.13 & 15.12 & 0.01 & 50.05 & 0.20 \\
\hline
\end{tabular}

In Table 6, the grand average and standard deviation of $\mathrm{Cp}$ is $15.15 \pm 0.37$ and of $T_{m}$ is $(49.97 \pm 0.08)^{\circ} \mathrm{C}$.

Scrutiny of the data: two suspicious data were detected (in bold), and no trend was observed ( $t$-test; $t_{0.05,22}$, data not shown). The outlying data were however retained in the evaluation, because here no distinction could be made between the influence of statistical variation and inhomogeneity of the batch.

All average $T_{m}$ values $\pm s$ of the individual samples (Table 6 ) are within the expected interval mentioned in the kit manual $(49 \pm 2.5){ }^{\circ} \mathrm{C}$. Therefore, all samples could be categorised as having the G20210A mutant genotype. Moreover, since the batch has been prepared by dilution from a stock solution that had been sequenced and since all samples are liquid (frozen at $-20^{\circ} \mathrm{C}$ ), heterogeneity is unlikely to be found.

Analysis of the data by ANOVA on all $\mathrm{Cp}$ values is summarized in Table 7 (using MS Excel $^{\mathrm{TM}}$ Single Factor ANOVA). 
Table 7. Analysis of data from Table 6 using MS Excel ${ }^{\mathrm{TM}}$ Single Factor ANOVA

\begin{tabular}{|l|r|r|r|}
\hline & SS & v & MS \\
\hline Among vials & 6.399 & 23 & 0.278 \\
\hline Within vials & 0.939 & 24 & 0.039 \\
\hline Total & 7.338 & 47 & \\
\hline
\end{tabular}

From Table 7 the repeatability of the procedure was calculated as $1.31 \%$, according to the procedures described in references [1-4]. The $s_{\mathrm{bb}}$ was calculated as $8.88 \%$ and the relative standard uncertainty $u_{b b}{ }^{*}$ was calculated as $0.50 \%$. The maximum relative standard uncertainty contribution to homogeneity was therefore $8.88 \%$.

A plot of the $\mathrm{Cp}$ values of the PCR products versus the sample number did not reveal a trend in the filling sequence (data not shown). The slope of the regression line was not statistically different from zero ( $t$-test, data not shown) and the data followed a normal and unimodal distribution (data not shown).

In conclusion, the batch IRMM/IFCC-491 produced is homogeneous and contains the G20210A mutant sequence of a 609 bp fragment of the human prothrombin gene. 


\section{Stability}

\subsection{Planning}

The stability studies have been assessed by isochronous measurements at time points of 2, 4 and 8 weeks for the short-term, and at 2, 4 and 6 months for the longterm study. The reference temperature was set at $-70^{\circ} \mathrm{C}$, corresponding to time point 0 .

\subsection{Short-term stability}

In order to assess the stability of the material, an indication of the pDNA quantity in each vial was investigated using the $\mathrm{Cp}$ values obtained after the PCR reaction in the LightCycler. Six different vials were tested in duplicate ( $5 \mu \mathrm{L}$ pDNA per PCR reaction) at each temperature. As the $\mathrm{Cp}$ values give an indication of the quantity of the pDNA remaining after storage, the melting temperatures allow genotyping of the DNA fragments (Tables 8.1 and 8.2).

Table 8.1: Short-term stability of IRMM/IFCC-491 stored for 8 weeks at $-70{ }^{\circ} \mathrm{C}$

\begin{tabular}{|l|c|c|}
\hline & Cp values & $\mathbf{T}_{\mathbf{m}}$ values \\
\hline Sample 1 & 15.33 & 49.96 \\
\hline Sample 2 & 15.35 & 50.01 \\
\hline Sample 3 & 15.70 & 49.94 \\
\hline Sample 4 & 15.25 & 49.88 \\
\hline Sample 5 & 15.43 & 49.88 \\
\hline Sample 6 & 15.45 & 49.92 \\
\hline Average & 15.41 & 49.93 \\
\hline $\mathrm{s}$ & 0.16 & 0.08 \\
\hline
\end{tabular}

$C p$ and $T_{m}$ values represent respectively the averages of $C p$ and $T_{m}$ values of a duplicate measurement of samples stored at the reference temperature in the isochronous study investigating the stability at $-20^{\circ} \mathrm{C}, 4^{\circ} \mathrm{C}$ and $18^{\circ} \mathrm{C}$.

The grand average and standard deviation of the $C p$ values and $T_{m}$ values of samples stored at $-70{ }^{\circ} \mathrm{C}$ is respectively $\mathrm{Cp}=15.41 \pm 0.16$ and $\mathrm{T}_{\mathrm{m}}=(49.93 \pm 0.08)$ ${ }^{\circ} \mathrm{C}$.

The samples of Table 8.1 were analysed simultaneously with those in Table 8.2 as they represented the samples stored at the reference temperature in the stability study. 
Table 8.2: Short-term stability of IRMM/IFCC-491 stored for 2, 4 and 8 weeks at $-20{ }^{\circ} \mathrm{C}, 4^{\circ} \mathrm{C}$ and $18{ }^{\circ} \mathrm{C}$. Suspicious data are in bold. The * indicates values for which no replicate was obtained.

\begin{tabular}{|l|c|c|c|c|}
\hline & & \multicolumn{3}{|c|}{ Cp values at $\boldsymbol{t}$ (week) } \\
\hline & $\begin{array}{l}\text { Temperature } \\
{ }^{\circ} \text { C }\end{array}$ & $\boldsymbol{t}=\mathbf{2}$ & $\boldsymbol{t}=\mathbf{4}$ & $\boldsymbol{t}=\mathbf{8}$ \\
\hline Sample 1 & -20 & 15.18 & 15.27 & 15.06 \\
\hline Sample 2 & & 15.54 & 13.69 & 14.87 \\
\hline Sample 3 & & 15.22 & 15.23 & 15.02 \\
\hline Sample 4 & & 15.30 & 15.03 & 15.05 \\
\hline Sample 5 & & 15.41 & 14.94 & 15.17 \\
\hline Sample 6 & & 15.11 & 14.90 & 15.12 \\
\hline Average & & 15.29 & 14.84 & 15.05 \\
\hline s & & 0.16 & 0.59 & 0.10 \\
\hline Sample 1 & 4 & 14.55 & 14.94 & 14.25 \\
\hline Sample 2 & & 14.90 & 14.92 & 14.49 \\
\hline Sample 3 & & 14.87 & 14.95 & 14.88 \\
\hline Sample 4 & & 15.05 & 15.06 & 14.69 \\
\hline Sample 5 & & 14.56 & 14.84 & 14.75 \\
\hline Sample 6 & & 14.93 & $15.12^{\star}$ & 14.13 \\
\hline Average & & 14.81 & 14.96 & 14.53 \\
\hline s & & 0.21 & 0.10 & 0.09 \\
\hline Sample 1 & 18 & 14.25 & 13.91 & 12.08 \\
\hline Sample 2 & & 14.49 & 14.05 & 13.47 \\
\hline Sample 3 & & 14.88 & 14.56 & 13.61 \\
\hline Sample 4 & & $14.69^{\star}$ & 13.81 & 13.21 \\
\hline Sample 5 & & 0.30 & 13.82 & 13.28 \\
\hline Sample 6 & & 14.53 & 14.00 & 12.73 \\
\hline Average & & 0.29 & 1.10 \\
\hline s & & & & 10.76 \\
\hline
\end{tabular}

The grand average and standard deviation of $\mathrm{Cp}$ values of the vials stored at $-20{ }^{\circ} \mathrm{C}$ is $\mathrm{Cp}=15.06 \pm 0.38, \mathrm{Cp}=14.86 \pm 0.16$ at $4{ }^{\circ} \mathrm{C}$ and $\mathrm{Cp}=13.75 \pm 1.01$ at $18{ }^{\circ} \mathrm{C}$ (Table 8.2).

Scrutiny of the data: two suspicious data (indicated in bold) were detected with a discrepancy of more than $50 \%$ of the average between vials. The outlying data (one at $-20^{\circ} \mathrm{C}$ and one at $18{ }^{\circ} \mathrm{C}$ ) were considered in the evaluation, because no distinction can be made between the influence of statistical variation and inhomogeneity of the batch. 
For the three temperatures the slope of the regression line and its uncertainty were calculated and tested for statistical significance (Figure 4).

At $-20^{\circ} \mathrm{C}$, the slope of the regression line was not significantly different from zero $(t-$ test, data not shown). At $4{ }^{\circ} \mathrm{C}$ and $18{ }^{\circ} \mathrm{C}$, the slopes of regression lines were significantly different from zero $\left(t\right.$-test; $\left.t_{0.05}, 23\right)$. As the concentration of the material was slightly increasing with time (i.e. decreasing $\mathrm{Cp}$ values), the regression line was technically insignificant.

The recovery of the material was increasing, due to evaporation of the solution with storage time. This phenomenon did not jeopardize the correct genotyping of IRMM/IFCC-491 after storage at these temperatures (Table 9).
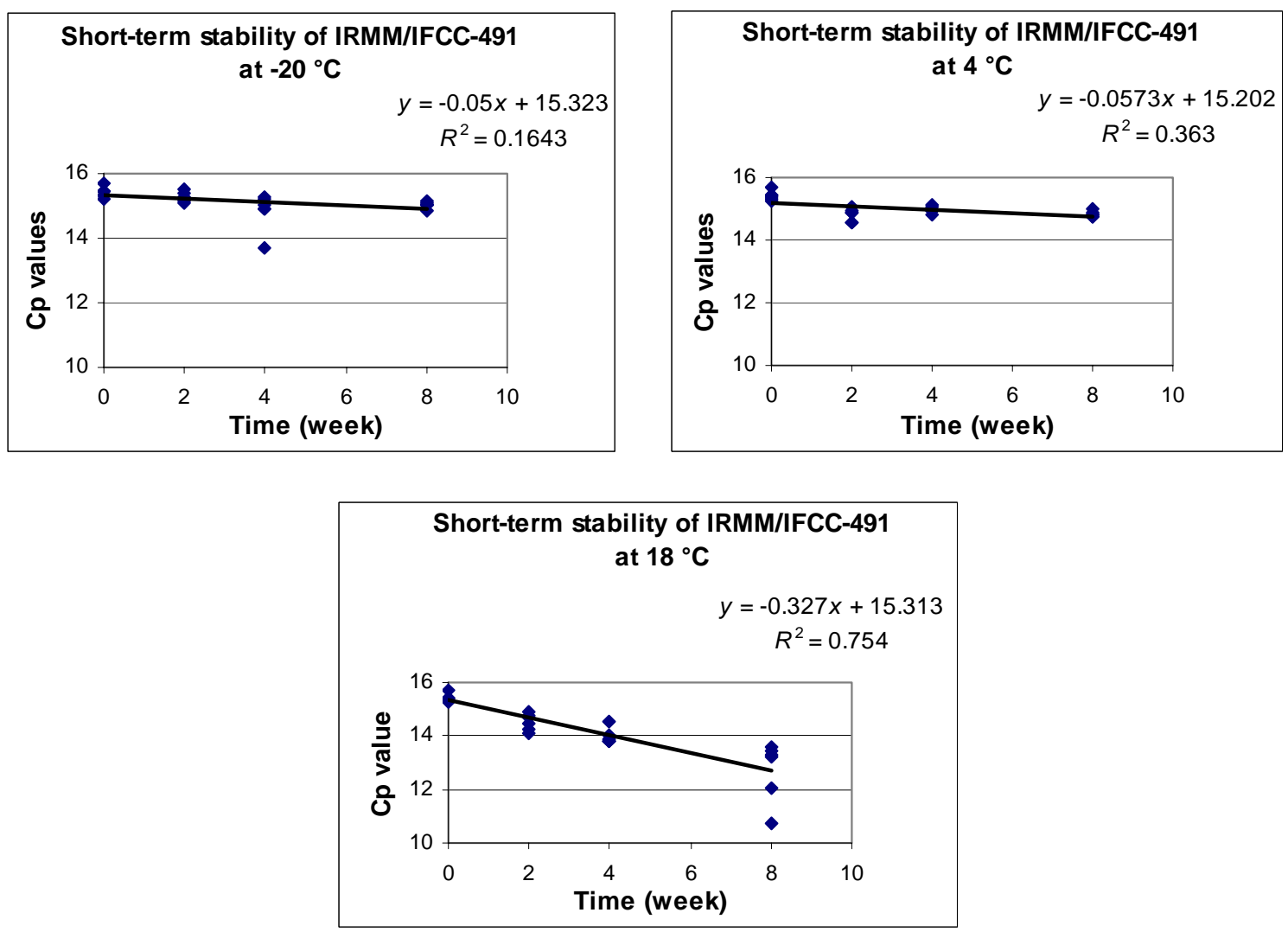

Figure 4. Slopes of the regression lines of IRMM/IFCC-491 during short-term stability study $\left(0,2,4\right.$ and 8 weeks at $-20^{\circ} \mathrm{C}, 4^{\circ} \mathrm{C}$ and $\left.18^{\circ} \mathrm{C}\right)$ 
Table 9: Melting temperature values of short-term stability samples of

IRMM/IFCC-491 stored for 2,4 and 8 weeks at $-20^{\circ} \mathrm{C}, 4^{\circ} \mathrm{C}$ and $18^{\circ} \mathrm{C}$.

\begin{tabular}{|c|c|c|c|c|}
\hline & \multicolumn{4}{|c|}{$T_{m}$ values at $t$ (week) } \\
\hline & $\begin{array}{l}\text { Temperature } \\
{ }^{\circ} \mathrm{C}\end{array}$ & $t=2$ & $t=4$ & $t=8$ \\
\hline Sample 1 & -20 & 49.96 & 49.94 & 50.10 \\
\hline Sample 2 & & 49.94 & 50.05 & 50.11 \\
\hline Sample 3 & & 49.96 & 50.14 & 50.06 \\
\hline Sample 4 & & 49.95 & 49.97 & 50.03 \\
\hline Sample 5 & & 49.90 & 49.87 & 50.05 \\
\hline Sample 6 & & 49.83 & 49.92 & 50.05 \\
\hline Average & & 49.92 & 49.98 & 50.07 \\
\hline$s$ & & 0.08 & 0.11 & 0.16 \\
\hline Sample 1 & 4 & 50.08 & 49.91 & 50.03 \\
\hline Sample 2 & & 50.13 & 49.93 & 50.04 \\
\hline Sample 3 & & 50.09 & 49.97 & 50.01 \\
\hline Sample 4 & & 50.0 & 49.95 & 49.93 \\
\hline Sample 5 & & 49.95 & 49.89 & 49.94 \\
\hline Sample 6 & & 50.0 & 49.90 & 49.95 \\
\hline Average & & 50.04 & 49.92 & 49.98 \\
\hline$s$ & & 0.08 & 0.07 & 0.06 \\
\hline Sample 1 & 18 & 49.94 & 50.11 & 49.99 \\
\hline Sample 2 & & 49.98 & 50.07 & 50.20 \\
\hline Sample 3 & & 50.04 & 50.18 & 50.08 \\
\hline Sample 4 & & 49.97 & 49.07 & 49.98 \\
\hline Sample 5 & & 49.92 & 49.95 & 49.97 \\
\hline Sample 6 & & 49.92 & 49.94 & 50.05 \\
\hline Average & & 49.96 & 50.03 & 50.04 \\
\hline$s$ & & 0.08 & 0.13 & 0.11 \\
\hline
\end{tabular}

In Table 9, each value represents an average of a duplicate. The average of six samples for each combination time/temperature shows that all $T_{m}$ values (Table 9) are within the interval of the expected value and standard deviation mentioned in the kit manual $\left((49 \pm 2.5){ }^{\circ} \mathrm{C}\right.$, Roche Diagnostics, Vilvoorde, Belgium).

Therefore, all samples could be categorised as having the G20210A mutant genotype. 
In conclusion, the batch IRMM/IFCC-491 is stable at $-20{ }^{\circ} \mathrm{C}$ and contains the G20210A mutant sequence of a 609 bp fragment of the human prothrombin gene, that can still be identified in samples which were stored at higher temperature such as $18^{\circ} \mathrm{C}$ for up to 8 weeks.

\subsection{Long-term stability}

At temperature of $18{ }^{\circ} \mathrm{C}$ evaporation occurred already after 2 months, therefore the long-term stability was not investigated at this temperature. The long-term stability study was performed at $-20{ }^{\circ} \mathrm{C}$ and at $4{ }^{\circ} \mathrm{C}$ for time periods of 2,4 and 6 months (Tables 10.1 and 10.2). Six different vials were tested in triplicate $(5 \mu \mathrm{L}$ pDNA per PCR reaction) at each temperature by real time PCR and the triplicate average is displayed in Tables 10.1 and 10.2. As two different instruments (ABI Prism $7900 \mathrm{HT}$ and $A B I$ Prism 7700) were used to analyse the samples, an internal control was used on each plate to be able to compare the data, and therefore the average $\mathrm{Ct}$ value of each sample was normalised by the average value of the triplicate measurements of the control on each plate, giving $\mathrm{Ct}^{*}$ values. The average $\mathrm{Ct}$ values obtained using the $\mathrm{ABI}$ Prism 7700 instrument are displayed in bold. The average $\mathrm{Ct}$ value and standard deviation of the internal control (pDNA at $0.02 \mathrm{ng} / \mu \mathrm{L}$ ) was $15.78 \pm 0.25$ and $16.52 \pm 0.07$ using the $A B I 7900 \mathrm{HT}$ and $A B I$ Prism 7700 instruments, respectively.

Table 10.1. Long-term stability of IRMM/IFCC-491 stored for 6 months at $-70^{\circ} \mathrm{C}$. ** indicates a value for which the average was performed only on duplicates, due to an error in pipetting.

\begin{tabular}{|c|c|c|c|c|}
\hline & & \multicolumn{2}{|c|}{ Ct values } & \multirow{2}{*}{$\begin{array}{c}\mathbf{T}_{\mathrm{m}} \text { values } \\
{ }^{\circ} \mathrm{C}\end{array}$} \\
\hline & $\begin{array}{l}\text { Temperature } \\
{ }^{\circ} \mathrm{C}\end{array}$ & Ct & $\mathrm{Ct}^{*}$ & \\
\hline Sample 1 & -70 & 15.79 & 1.00 & 49.95 \\
\hline Sample 2 & & 15.68 & 0.99 & 50.01 \\
\hline Sample 3 & & $15.89^{* \star}$ & 0.98 & 50.01 \\
\hline Sample 4 & & $15.40^{* \star}$ & 0.98 & 49.98 \\
\hline Sample 5 & & 15.65 & 0.99 & 49.94 \\
\hline Sample 6 & & 15.52 & 0.98 & 50.14 \\
\hline Average & & 15.65 & 0.99 & 50.00 \\
\hline$S$ & & 0.18 & 0.01 & 0.11 \\
\hline \multicolumn{2}{|c|}{ Control ABI 7900HT } & 15.78 & - & - \\
\hline
\end{tabular}

The data of Table 10.1 were analysed simultaneously with those in Table 10.2 as they represented the samples stored at the reference temperature. The grand average and standard deviation of the $\mathrm{Ct}^{\star}$ values and $\mathrm{T}_{\mathrm{m}}$ values of samples stored at 
$-70{ }^{\circ} \mathrm{C}$ is respectively $0.99 \pm 0.01$ and $(50.00 \pm 0.11)^{\circ} \mathrm{C}$. The $T_{m}$ values of Table 10.1 constitute the values obtained at the reference temperature for samples analysed in Table 11.

Scrutiny of the data: no suspicious data were detected.

Table 10.2. Long-term stability of IRMM/IFCC-491 stored for 2, 4 and 6 months at two different temperatures. The average $\mathrm{Ct}$ values obtained using the $\mathrm{ABI}$ Prism 7700 instrument are displayed in bold. ** indicates a value for which the average was performed only on duplicates, due to an error in pipetting.

\begin{tabular}{|c|c|c|c|c|c|c|c|}
\hline & & \multicolumn{6}{|c|}{ Ct values at $t$ (month) } \\
\hline & \multirow{2}{*}{$\begin{array}{l}\text { Temperature } \\
{ }^{\circ} \mathrm{C}\end{array}$} & \multicolumn{2}{|c|}{$t=2$} & \multicolumn{2}{|c|}{$t=4$} & \multicolumn{2}{|c|}{$t=6$} \\
\hline & & Ct & $\mathbf{C t}^{*}$ & Ct & $\mathrm{Ct}^{*}$ & Ct & $\mathbf{C t}^{*}$ \\
\hline Sample 1 & -20 & 15.49 & 0.98 & 15.53 & 0.98 & 15.56 & 0.99 \\
\hline Sample 2 & & 15.64 & 0.99 & 15.58 & 0.99 & $15.61^{* \star}$ & 0.99 \\
\hline Sample 3 & & 15.71 & 1.00 & 15.53 & 0.98 & 15.82 & 1.00 \\
\hline Sample 4 & & $15.62^{\star \star}$ & 0.97 & 15.70 & 1.00 & 15.72 & 1.00 \\
\hline Sample 5 & & 15.72 & 1.00 & 15.63 & 0.99 & 15.46 & 0.98 \\
\hline Sample 6 & & 15.88 & 1.01 & 15.81 & 1.00 & 15.43 & 0.98 \\
\hline Average & & 15.68 & 0.99 & 15.63 & 0.99 & 15.60 & 0.99 \\
\hline$s$ & & 0.13 & 0.01 & 0.11 & 0.01 & 0.15 & 0.01 \\
\hline \multicolumn{2}{|c|}{ Control ABI 7900HT } & 15.78 & - & 15.78 & - & 15.78 & - \\
\hline Sample 1 & 4 & 15.45 & 0.98 & 16.61 & 1.00 & 16.39 & 0.99 \\
\hline Sample 2 & & 15.42 & 1.00 & 16.28 & 0.99 & 16.30 & 0.99 \\
\hline Sample 3 & & 15.49 & 0.98 & 16.43 & 0.99 & 16.39 & 0.99 \\
\hline Sample 4 & & 15.52 & 0.98 & 16.46 & 1.00 & 16.29 & 0.99 \\
\hline Sample 5 & & 15.71 & 1.01 & 16.30 & 0.99 & 16.29 & 0.99 \\
\hline Sample 6 & & $15.55^{\star *}$ & 0.96 & 15.94 & 0.97 & 16.27 & 0.99 \\
\hline Average & & 15.69 & 0.99 & 16.34 & 0.99 & 16.32 & 0.99 \\
\hline$s$ & & 0.37 & 0.03 & 0.23 & 0.01 & 0.05 & 0.00 \\
\hline \multicolumn{2}{|c|}{ Control ABI 7900HT } & $15.78^{\star \star}$ & - & - & - & - & - \\
\hline \multicolumn{2}{|c|}{ Control ABI 7700} & - & - & 16.52 & - & 16.52 & - \\
\hline
\end{tabular}

Scrutiny of the data: no suspicious data were detected.

For the two temperatures, the slope of the regression line using the $\mathrm{Ct}^{*}$ values (Figures $5 \mathrm{~A}$ and $5 \mathrm{~B}$ ) and its uncertainty were calculated and tested for statistical significance ( $t$-test; $\left.t_{0.05,22}\right)$. In both cases, the slope was not significantly different from zero (data not shown). 
Figure $5 \mathrm{~A}$ : at $-20^{\circ} \mathrm{C}$

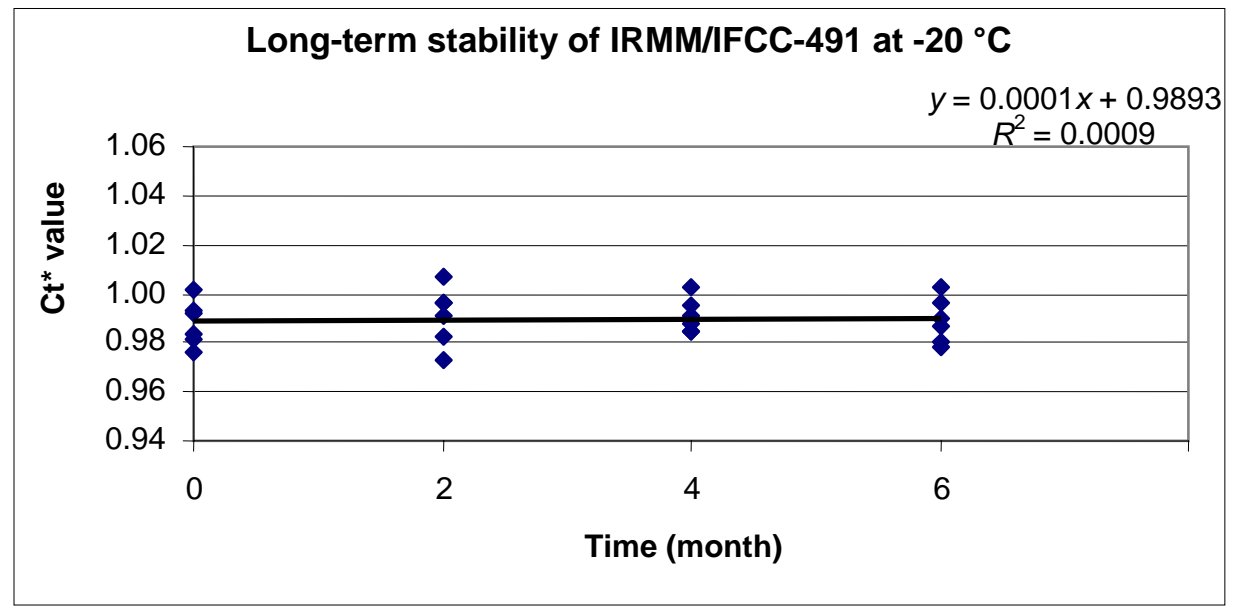

Figure 5B: at $4{ }^{\circ} \mathrm{C}$

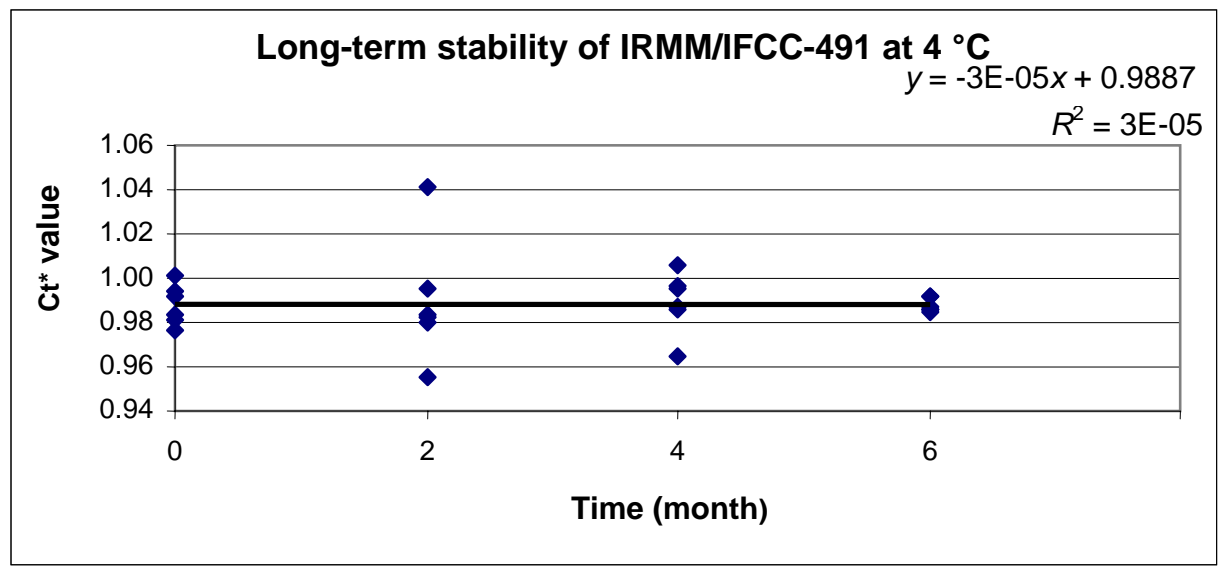

Figure 5. Slopes of the regression lines of IRMM/IFCC-491 during long-term stability study $\left(0,2,4\right.$ and 6 months at $-20{ }^{\circ} \mathrm{C}(\mathrm{A})$ and $\left.4{ }^{\circ} \mathrm{C}(\mathrm{B})\right)$

Additionally, the melting temperatures of the PCR products were recorded for genotyping purposes (Table 11). The grand average and standard deviation of the $T_{m}$ values of samples stored at $-20^{\circ} \mathrm{C}$ is $(50.69 \pm 0.51){ }^{\circ} \mathrm{C}$ and $(50.11 \pm 0.31){ }^{\circ} \mathrm{C}$ at $4{ }^{\circ} \mathrm{C}$. All $\mathrm{T}_{\mathrm{m}}$ values of the individual samples (Tables 10.1 and 11) are within the interval of the expected value mentioned in the kit manual $\left((49 \pm 2.5){ }^{\circ} \mathrm{C}\right.$, Roche Diagnostics, Vilvoorde, Belgium).

Therefore, all samples could be categorised as having the G20210A mutant genotype.

In conclusion, the batch of IRMM/IFCC-491 is stable for 6 months at $-20{ }^{\circ} \mathrm{C}$ and at 4 ${ }^{\circ} \mathrm{C}$ and contains the G20210A mutant sequence of a $609 \mathrm{bp}$ fragment of the human prothrombin gene. 
Table 11: Melting temperature $l^{\circ} \mathrm{C}$ of long-term stability samples of IRMM/IFCC491 stored for 2, 4 and 6 months at two different temperatures

\begin{tabular}{|l|l|c|c|c|}
\hline & & \multicolumn{3}{|c|}{$T_{\mathbf{m}}$ values at $\boldsymbol{t}$ (week) } \\
\hline & $\begin{array}{l}\text { Temperature } \\
{ }^{\circ} \mathrm{C}\end{array}$ & $\boldsymbol{t}=\mathbf{2}$ & $\boldsymbol{t}=\mathbf{4}$ & $\boldsymbol{t}=\mathbf{6}$ \\
\hline Sample 1 & -20 & 50.95 & 50.10 & 50.85 \\
\hline Sample 2 & & 51.49 & 50.07 & 50.81 \\
\hline Sample 3 & & 51.32 & 50.12 & 50.74 \\
\hline Sample 4 & & 51.15 & 50.01 & 50.79 \\
\hline Sample 5 & & 51.21 & 50.01 & 50.85 \\
\hline Sample 6 & & 51.28 & 50.00 & 50.75 \\
\hline Average & & 51.23 & 50.05 & 50.80 \\
\hline S & & 0.18 & 0.05 & 0.05 \\
\hline Sample 1 & 4 & 50.49 & 49.78 & 49.97 \\
\hline Sample 2 & & 50.42 & 49.75 & 50.05 \\
\hline Sample 3 & & 50.50 & 49.80 & 50.19 \\
\hline Sample 4 & & 50.37 & 49.80 & 50.17 \\
\hline Sample 5 & & 50.46 & 49.68 & 50.10 \\
\hline Sample 6 & & 50.34 & 49.70 & 50.51 \\
\hline Average & & 50.43 & 49.75 & 50.16 \\
\hline s & & 0.07 & 0.05 & 0.19 \\
\hline
\end{tabular}




\section{Batch characterisation}

After plasmid construction and production, the cloned human prothrombin (Factor II) fragment in pUC18 plasmid was sequenced using DTCS protocol [10] (see [6]).

The correct G20210A mutation sequence was confirmed at position 26784 for the IRMM/IFCC-491 material. Another point mutation A->G was detected at position 26628, which was confirmed to be present as well in the patient sample. This mutation is natural and an introduction due to in vitro manipulations could be excluded.

\subsection{Sequencing}

The $609 \mathrm{bp}$ fragment of the human prothrombin was sequenced using sequencing forward primers M13-F (P-0419) and Factor II-F (P-0579) and reverse primers Factor II-R (P-0580) and M13-R (P-0289) (Table 5), and DTCS protocol [10].

Forward and reverse sequence matched, indicating a very low probability of misreadings $\left(p<3 \times 10^{-6}\right)$. As there was no error in the sequencing of the insert, the misreading probability was calculated as to be inferior to $1 / 609$ for the forward sequencing reaction, and inferior to 1/609 when backward sequencing was performed. Therefore, the total misreadings probability in the certified sequence was calculated as inferior to $1 / 609 \times 1 / 609$.

The analysed fragment of prothrombin DNA sequence showed $100 \%$ homology with the reported DNA sequence of the prothrombin gene (GeneBank Accession number M17262), confirming the identity of produced pDNA (Figure 6) except for two point mutations, the G20210A mutation at position 26784 in the reference sequence and the A20054G point mutation at position 26628 in the reference sequence. This sequence was also confirmed by sequencing using the U-20 universal primer and an ABI 377 instrument by Eurogentec (Liège, Belgium).

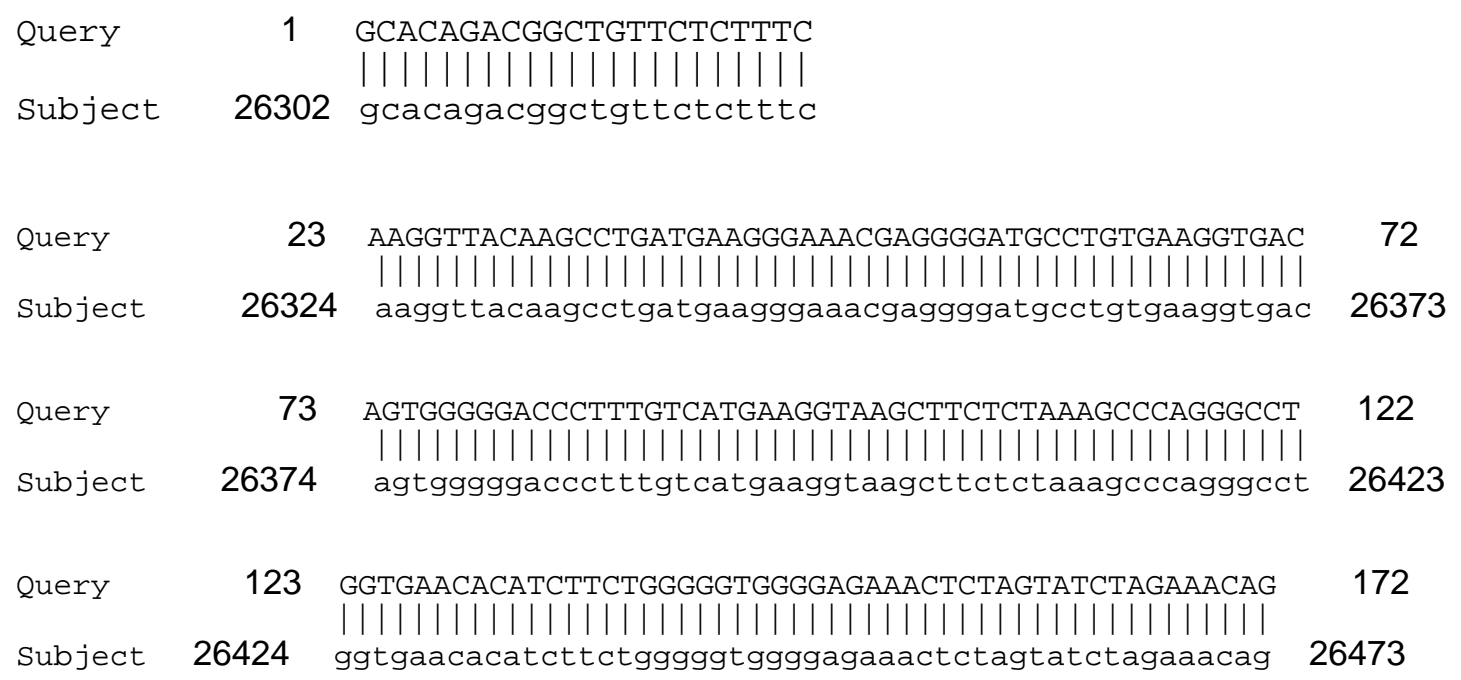




\begin{tabular}{|c|c|c|}
\hline Query & 173 & TTGCCTGGCAGAGGAATACTGATGTGACCTTGAACTTGACTCTATTGGAA \\
\hline Subject & 26474 & ttgcctggcagaggaatactgatgtgaccttgaacttgactctattggaa \\
\hline Query & 223 & 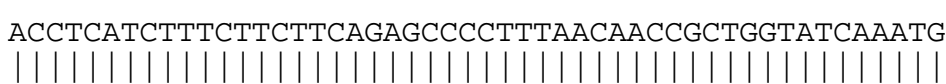 \\
\hline Subject & 26524 & acctcatcttcttcttcagagcccettaacaaccgctggtatcaaatg \\
\hline Query & 273 & $\begin{array}{l}\text { GGCATCGTCTCATGGGGTGAAGGCTGTGACCGGGATGGGAAATATGGCTT } \\
\text { T }\end{array}$ \\
\hline Subject & 26574 & ggcatcgtctcatggggtgaaggctgtgaccgggatgggaaatatggctt \\
\hline Query & 323 & GAAGTGGATACAGAAGGTCATTGATC \\
\hline Subject & 26624 & ctacacacatgtgttccgcctgaagaagtggatacagaaggtcattgatc \\
\hline Query & 373 & TTGGAGAGTAGGGGGCCACTCATATTCTGGGCTCCTGGAACCAATCC \\
\hline Subject & 26674 & agtttggagagtagggggccactcatattctgggctcctggaaccaatcc \\
\hline Query & 423 & $\begin{array}{l}\text { CGTGAAAGAATTATTTTTGTGTTTCTAAAACTATGGTTCCCAATAAAAGT } \\
\text { C. }\end{array}$ \\
\hline Subject & 26724 & cgtgaaagaattatttttgtgtttctaaaactatggttcccaataaaagt \\
\hline Query & 473 & TCTCAGCAAGCCTCAATGCTCCCAGTGCTATTCATGGGCAGCTCTCT \\
\hline Subject & 26774 & gactctcagcgagcctcaatgctcccagtgctattcatgggcagctctct \\
\hline Query & 523 & 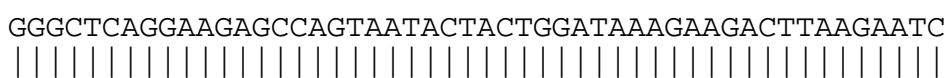 \\
\hline Subject & 26824 & gggctcaggaagagccagtaatactactggataaagaagacttaagaatc \\
\hline Query & 573 & $\begin{array}{l}\text { CACCACCTGGTGCACGCTGGTAGTCCGAGCACTCGGG } \\
\text { CACATG }\end{array}$ \\
\hline Subject & 26874 & caccacctggtgcacgctggtagtccgagcactcggg \\
\hline
\end{tabular}

Figure 6. Comparison of the homology of the DNA sequence of the $609 \mathrm{bp}$ fragment of human prothrombin obtained from IRMM/IFCC-491 production batch (Query) with the reported DNA sequence (Subject) of the prothrombin gene (GeneBank accession number M17262) using BLAST 2 sequences on www.ncbi.nlm.nih.gov. Mutations are highlighted in bold.

In conclusion, the correct G20210A mutant sequence was confirmed for the IRMM/IFCC-491 material, with one extra mutation that was already present in the patient. The sequencing method is the most robust and straightforward one to determine a DNA sequence, but requires a rather large minimum amount of DNA and is relatively time consuming. When only a lower DNA quantity is available, it can only be detected by using PCR-based methods such as real-time PCR, hybridisation techniques and DNA profiling. 


\section{Validation}

\subsection{Field study design and results}

The performance of the material was tested in a field study by 7 diagnostic laboratories using 4 different methods routinely for the G20210A prothrombin mutation detection (Table 12). A total of eight vials labeled from $\mathrm{A}$ to $\mathrm{H}$ was sent to each laboratory. Each set of vials contained two vials of each genotype: IRMM/IFCC490 (wildtype sequence), IRMM/IFCC-491 (G20210A mutant sequence) or IRMM/IFCC-492 (heterozygous wildtype/G20210A mutant sequence generated by mixing of equal volumes of identical mass concentrations of IRMM/IFCC-490 and IRMM/IFCC-491), and two vials without DNA (Blank). The testing laboratories were provided with the study protocol, the terms to be used for reporting and the results reporting form, but had no information on the expected results or identity of the individual samples. All laboratories were asked to describe in detail the method used.

Table 12: Field study of IRMM/IFCC-490, -491 and -492 materials (Y: Yes, N: No)

\begin{tabular}{|c|c|c|c|c|c|c|}
\hline Lab & Method & Type & Correctness & f genotyping & & \\
\hline & & & $\begin{array}{l}\text { Homozygous } \\
\text { wildtype }\end{array}$ & $\begin{array}{l}\text { Homozygous } \\
\text { mutant }\end{array}$ & $\begin{array}{l}\text { Heterozygous } \\
\text { wildtype/G20210A } \\
\text { mutant }\end{array}$ & Blank \\
\hline 1 & PCR & LightCycler & $Y$ & $Y$ & $Y$ & $Y$ \\
\hline 2 & $\begin{array}{l}\text { Multiplex } \\
\text { PCR }\end{array}$ & Home brewed & Y & $Y$ & $Y$ & Y \\
\hline 3 & $\begin{array}{l}\text { Multiplex } \\
\text { PCR }\end{array}$ & Home brewed & $Y$ & $Y$ & $Y$ & Y \\
\hline 4 & PCR & LightCycler & $Y$ & $Y$ & $Y$ & $\mathrm{Y}$ \\
\hline 5 & RFLP1 & Home brewed & $Y$ & $Y$ & $Y$ & Y \\
\hline 5 & PCR & LightCycler & $Y$ & $Y$ & $Y$ & $\mathrm{~N}$ \\
\hline 6 & RFLP2 & Home brewed & $Y$ & $Y$ & $Y$ & $Y$ \\
\hline 7 & PCR & Rotor-Gene & Y & $Y$ & $Y$ & Y \\
\hline
\end{tabular}

In Table 12, each $\mathrm{Y}$ or $\mathrm{N}$ represents agreement on the two samples that have been tested by the corresponding laboratory. All DNA-containing samples were genotyped correctly. Two vials without DNA were misclassified (one bold $\mathbf{N}$ representing the agreement on two blank samples). These data were reported as homozygous wildtype by laboratory number 5 using the LightCycler method. The same samples, tested with the in-house RFLP method by the same laboratory, were correctly identified as blank samples. The use of two different methods by laboratory number 5 was agreed before starting the study. In laboratory number 3, the samples were diluted 1:20 before use, but the dilution of the material did not influence the genotyping results. 
All vials containing pDNA (wildtype, mutant or heterozygous wildtype/G20210A mutant) were genotyped correctly and unambiguously demonstrating that the material is fit for its intended purpose.

\subsection{Methods}

Roche LightCycler: LightCycler technology from Roche, using the Factor II (prothrombin) G20210A kit and LightCycler 1.5 instrument [5].

Multiplex mutagenic separated PCR: derived from Endler et al. [11]

Thermal PCR program: $35 \times$ cycles of $95{ }^{\circ} \mathrm{C}$ for $1 \mathrm{~min} ; 54{ }^{\circ} \mathrm{C}$ for $2 \mathrm{~min} ; 72{ }^{\circ} \mathrm{C}$ for 1 min; then hold at $4{ }^{\circ} \mathrm{C}$. Primers used are described hereafter:

\begin{tabular}{|l|l|}
\hline Forward (wildtype), F2G & 5'-CAC TGG GAG CAT TGA GGC GC -3' \\
\hline Forward (mutant), F2A & 5'-ATG AAT AGT AAT GGG AGC ATT GAG GAT T -3' \\
\hline Reverse & 5'- ATG TGT TCC GCC TGA AGA AGT GGA -3' \\
\hline
\end{tabular}

PCR products $(6 \mu \mathrm{L})$ were loaded on Spreadex EL 600 gels, run at $120 \mathrm{~V}$ for $2 \mathrm{~h}$, then for $20 \mathrm{~min}$ at $40{ }^{\circ} \mathrm{C}$ to $50^{\circ} \mathrm{C}$. Staining of the gels performed with $1: 20000$ diluted SYBRGreen for $30 \mathrm{~min}$ at room temperature.

RFLP1: Derived from Poort et al. [12]

Thermal PCR program: $95^{\circ} \mathrm{C}$ for $4 \mathrm{~min}, 32 \times$ cycles of $94{ }^{\circ} \mathrm{C}$ for $1 \mathrm{~min}$; $55^{\circ} \mathrm{C}$ for 1 min; $67^{\circ} \mathrm{C}$ for $1 \mathrm{~min}$; then hold at $4{ }^{\circ} \mathrm{C}$. Primers used are described hereafter:

\begin{tabular}{|l|l|}
\hline Forward & 5'-TCT AGA AAC AGT TGC CTG GC-3' \\
\hline Reverse & 5'-ATA GCA CTG GGA GCA TTG AAG C -3' \\
\hline
\end{tabular}

PCR products are digested with HindIII restriction enzyme and loaded on agarose gel for electrophoresis.

RFLP2: Derived from Danneberg et al. [13]

Thermal PCR program: $94{ }^{\circ} \mathrm{C}$ for $5 \mathrm{~min}, 35 \mathrm{x}$ cycles of $93^{\circ} \mathrm{C}$ for $1 \mathrm{~min} ; 58^{\circ} \mathrm{C}$ for 1 min; $72{ }^{\circ} \mathrm{C}$ for $1 \mathrm{~min} ; 72{ }^{\circ} \mathrm{C}$ for $3 \mathrm{~min}$, then hold at $4{ }^{\circ} \mathrm{C}$. Primers used are described hereafter:

\begin{tabular}{|l|l|}
\hline Forward & 5'-GCA CAG ACG GTG TTC TCT T -3' \\
\hline Reverse & 5'-ATA GCA CTG GGA GCA TTG AAG C -3' \\
\hline
\end{tabular}


PCR products are digested with HindIII restriction enzyme and loaded on agarose gel for electrophoresis.

Rotor-Gene real-time PCR analysis software 6.0: Factor II kit from Nuclear Laser Medicine, Settala, IT. 


\section{Traceability of gene fragment}

The sequence of the $609 \mathrm{bp}$ human prothrombin gene fragment inserted in the pUC18 vector to generate the IRMM/IFCC-491 material was assessed by the CEQ Dye Terminator Cycle Sequencing (including a pUC18 control plasmid) and confirmed by external sequencing by Eurogentec (Liège, Belgium) using the U-20 universal primer and the $\mathrm{ABI}$ Prism 377 instrument. The sequence identity is traceable to PCR-based sequencing methods with very low probability of misreadings $\left(p<3 \times 10^{-6}\right)$. 


\section{Value assignment}

There is no numerical value assignment for this reference material. The sequence at position 20210 is an A, and certified by using 2 different sequencing procedures. The uncertainty can be expressed as a probability that two procedures, independently performed in different laboratories, generated identical results by forward and backward sequencing $\left(\mathrm{p}<3 \times 10^{-6}\right)$. The misreading probability was calculated as to be inferior to $1 / 609$ for the forward sequencing reaction, and inferior to $1 / 609$ when backward sequencing was performed. Therefore, the total misreadings probability was calculated as inferior to $1 / 609 \times 1 / 609$.

Uncertainties related to homogeneity and stability studies did not jeopardize the presence of the target sequence, and it could be demonstrated that even after 8 weeks at $18{ }^{\circ} \mathrm{C}$ the material could still be used in genotyping experiments without impact on the performance of the qualitative methods applied for nucleotide identification. 


\section{Instructions for use}

\subsection{Dispatch}

Dispatch to the customer should be done with cooling elements. Upon receipt by the customer, the material should be kept at $-20^{\circ} \mathrm{C}$ for long-term storage or at $4{ }^{\circ} \mathrm{C}$ for use within eight weeks.

\subsection{Intended use}

This certified reference material (CRM) is intended to support the validation and the harmonisation of PCR-based methods used for detection of the G20210A mutation in the human prothrombin gene. In particular, this CRM is intended to be used as positive control material in diagnostic tests for the identification and detection of the G20210A mutation in the human prothrombin.

Use 0.5 to $5 \mu \mathrm{L}$ of pDNA solution per 20 to $50 \mu \mathrm{L}$ total volume of the PCR reaction (depending on the method and the instrument). Alternatively, the material can be diluted 10 times prior to use, in that case $5 \mu \mathrm{L}$ of the diluted sample should be used, and no storage can be guaranteed anymore.

The plasmid DNA as provided can be kept at $4{ }^{\circ} \mathrm{C}$ for eight weeks or $-20{ }^{\circ} \mathrm{C}$ for longterm storage. 


\section{References}

[1] Linsinger, T.P.J., Pauwels, J, van der Veen, A.M.H., Schimmel, H., Lamberty, A. (2001) Homogeneity and stability of reference materials. Accred Qual Assur 6, 20-25. [2] van der Veen, A.M.H., Linsinger, T.P.J., Pauwels, J. (2001) Uncertainty calculations in the certification of reference materials. 2. Homogeneity study. Accred Qual Assur 6, 26-30.

[3] van der Veen, A.M.H., Linsinger, T.P.J., Lamberty, A., Pauwels, J. (2001) Uncertainty calculations in the certification of reference materials 3 . Stability study. Accred Qual Assur 6, 257-263.

[4] van der Veen, A.M.H, Linsinger, T.P.J., Schimmel, H., Lamberty, A., Pauwels, J. (2001) Uncertainty calculations in the certification of reference materials 4. Characterisation and certification. Accred Qual Assur 6, 290-294.

[5] http://www.roche-diagnostics.com/ba rmd/rmd products genomics 02.html

[6] Klein, C.L., Marki-Zay, J., Corbisier, P., Gancberg, D., Cooper, S et al. (2005) Reference materials (RMs) for analysis of the human factor II (prothrombin) gene G20210A mutation. Clin Chem Lab Med 43(8), 862-868.

[7] Handbook of Fluorescent Probes and Research Products, Section 8.3. http://probes.invitrogen.com/handbook, Molecular Probes (2005).

[8] QIAGEN plasmid purification Handbook, 2 $2^{\text {nd }}$ Edition, 2003. http://www1.qiagen.com/literature/handbooks/

[9] Corbisier, P. Validation report Working Instruction RM WI/0090, Fluorescent quantitative determination of ds DNA by PicoGreen, Internal report IRMM (2004).

[10] CEQ 8000 Series - DTCS Chemistry Protocol. http://www.beckman.com/ Literature/BioResearch/390003ac.pdf. Beckman Coulter, Inc. (2003).

[11] Endler, G., Kyrle, P.A., Eichinger, S., Exner, M., Mannhalter, C. (2001) Multiplexed mutagenically separated PCR: simultaneous single-tube detection of the factor V R506Q (G1691A), the prothrombin G20210A, and the methylenetetrahydrofolate reductase A223V (C677T) variants. Clin Chem 47, 333335.

[12] Poort, S.R., Rosendaal, F.R., Reitsma, P.H., Bertina, R.M. (1996) A common genetic variation in the 39-untranslated region of the prothrombin gene is associated with elevated plasma prothrombin levels and increase in venous thrombosis. Blood 88, 3698-3703.

[13] Danneberg, J., Abbes, A.P., Bruggeman, B.J., Engel, H., Gerrits, J., Martens, A. (1998) Reliable genotyping of the G20210A mutation of coagulation factor II (prothrombin). Clin Chem 44, 349-351. 
EUR 22170 EN - DG Joint Research Centre, Institute for Reference Materials and Measurements Certification of a Reference Material consisting of purified plasmid DNA containing a fragment from the human prothrombin gene (G20210A mutant

Authors: D. Gancberg, J. Marki-Zay, P. Corbisier, C. Klein, H. Schimmel, H. Emons

Luxembourg: Office for Official Publications of the European Communities

$2006-35$ pp. $-21.0 \times 29.7 \mathrm{~cm}$

Scientific and Technical Research series

ISBN 92-79-01634-2

\begin{abstract}
This report describes the production and certification of a reference material consisting of purified plasmid DNA (pDNA) pUC18 containing a 609 base pairs fragment of human Factor II (prothrombin) gene sequence, IRMM/IFCC-491. This certified reference material (CRM) is intended to support the validation and the harmonisation of polymerase chain reaction (PCR)-based methods used for detection of the G20210A mutation in the human prothrombin gene. The homogeneity and short-term stability of the batch was assessed by monitoring the melting temperature $\left(\mathrm{T}_{\mathrm{m}}\right)$ of the product and the crossing point threshold (Cp) value obtained after PCR amplification using the LightCycler Factor II (prothrombin) G20210A kit and a LightCycler 1.5 instrument. The long-term stability was assessed using the cycle threshold value (Ct) obtained after real-time PCR amplification using specific primers and probe in combination with $\mathrm{ABI}$ Prism instruments.

The material is homogeneous and stable at $-20^{\circ} \mathrm{C}$. The sequence of the insert (G20210A mutation) was confirmed by DNA sequence analysis. Although a second mutation $(A->G)$ was found at position 20054 , its location did not influence the G20210A diagnostic protocol using the Factor II (prothrombin) G20210A kit. Each vial contains an indicative volume of $50 \mu \mathrm{L}$ corresponding to approximately $1 \mathrm{ng}$ of pDNA.
\end{abstract}


The mission of the Joint Research Centre is to provide customer-driven scientific and technical support for the conception, development, implementation and monitoring of European Union policies. As a service of the European Commission, the JRC functions as a reference centre of science and technology for the Community. Close to the policy-making process, it serves the common interest of the Member States, while being independent of special interests, whether private or national.

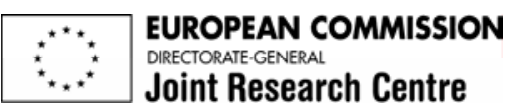




$$
\text { IV. }
$$


Certification of a Reference Material consisting of purified plasmid DNA containing a fragment from the human prothrombin gene (heterozygous G20210 wildtype/G20210A mutant)

Certified Reference Material IRMM/IFCC-492

D. Gancberg, J. Marki-Zay, P. Corbisier, C. Klein, H. Schimmel, H. Emons

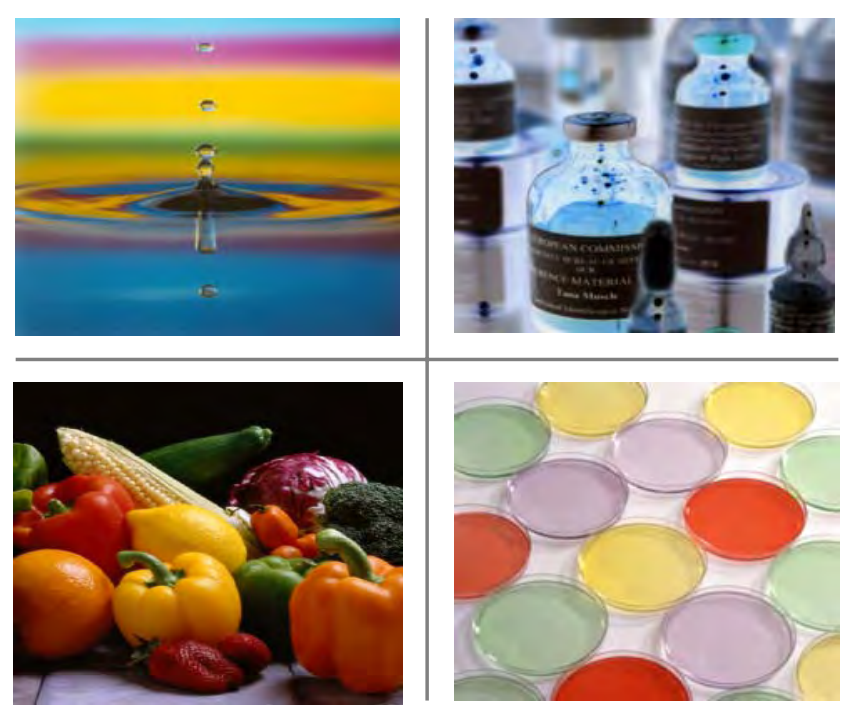


The mission of IRMM is to promote a common and reliable European measurement system in support of EU policies.

\section{European Commission}

Directorate-General Joint Research Centre

Institute for Reference Materials and Measurements

\section{Contact information}

\section{Gancberg}

European Commission

Directorate-General Joint Research Centre

Institute for Reference Materials and Measurements

Retieseweg 111

B-2440 Geel • Belgium

E-mail: david.gancberg@ec.europa.eu

Tel.: +32 (0)14 571911

Fax: +32 (0)14571 548

http://www.irmm.jrc.be

http://www.jrc.cec.eu.int

\section{Legal Notice}

Neither the European Commission nor any person acting on behalf of the Commission is responsible for the use which might be made of the following information.

A great deal of additional information on the European Union is available on the Internet. It can be accessed through the Europa server

http://europa.eu.int

\section{EUR Report 22167 EN}

Luxembourg: Office for Official Publications of the European Communities

ISBN 92-79-01632-6

ISSN 1018-5593

(c) European Communities, 2006

Reproduction is authorised provided the source is acknowledged 
IRMM information

REFERENCE MATERIALS

\title{
Certification of a Reference Material consisting of purified plasmid DNA containing a fragment from the human prothrombin gene (G20210A mutant)
}

\section{IRMM/IFCC-492}

D. Gancberg, J. Marki-Zay, P. Corbisier, H. Schimmel, H. Emons

European Commission

Joint Research Centre

Institute for Reference Materials and Measurements (IRMM)

Retieseweg 111, 2440 Geel, Belgium

\author{
C.Klein \\ Directorate General Joint Research Centre \\ Institute for Health and Consumer Protection \\ Via Fermi, 1 \\ IT-21020 Ispra (Varese), Italy
}




\section{SUMMARY}

This report describes the production and certification of IRMM/IFCC-492, a reference material consisting of a mix of equal volumes of identical mass concentrations of two plasmid DNA (pDNA) containing either the wildtype or the G20210A mutant sequence of a 609 base pairs fragment for the human Factor II (prothrombin) gene (IRMM/IFCC-490 and IRMM/IFCC-491). This certified reference material (CRM) is intended to support the validation and the harmonisation of polymerase chain reaction (PCR)-based methods used for detection of the G20210A mutation in the prothrombin gene. The homogeneity and short-term stability of the batch was assessed by monitoring the melting temperature $\left(T_{m}\right)$ of the product and the crossing point threshold $(\mathrm{Cp})$ value obtained after PCR amplification using the LightCycler Factor II (prothrombin) G20210A kit and a LightCycler 1.5 instrument. The long-term stability was assessed using the cycle threshold value $(\mathrm{Ct})$ obtained after real-time PCR amplification using specific primers and probe in combination with $A B I$ Prism instruments.

The material is homogeneous and stable at $-20^{\circ} \mathrm{C}$. The sequence of the insert in both plasmids (containing the wildtype sequence or the G20210A mutation) was confirmed by DNA sequence analysis. Although a second mutation $(A->G)$ was found at position 20054 in IRMM/IFCC-491, its location did not influence the G20210A diagnostic protocol using the Factor II (prothrombin) G20210A kit. Each vial contains an indicative volume of $50 \mu \mathrm{L}$ corresponding to approximately $1 \mathrm{ng}$ of pDNA. 


\section{TABLE OF CONTENTS}

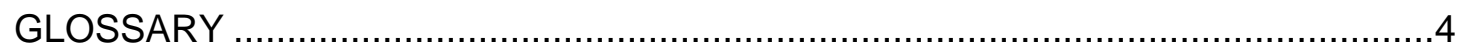

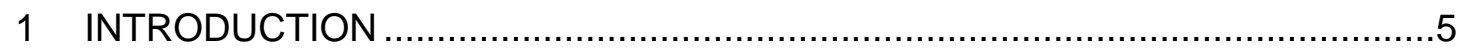

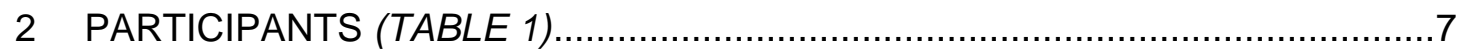

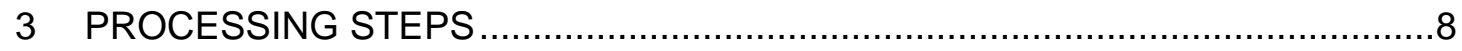

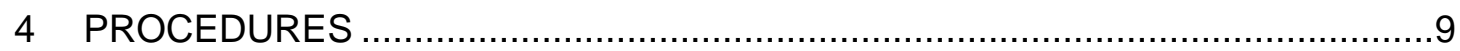

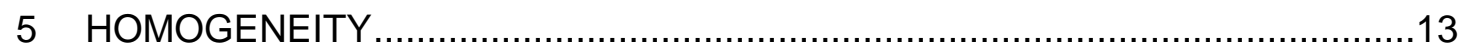

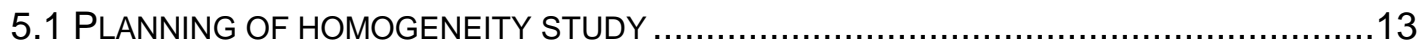

5.2 EVALUATION OF HOMOGENEITY STUDY ................................................... 13

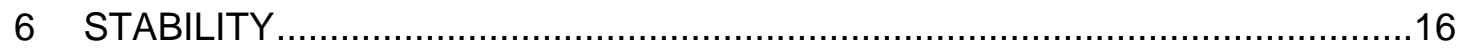

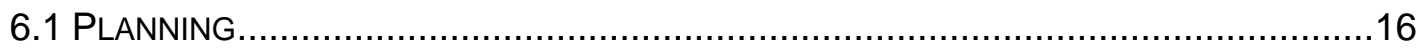

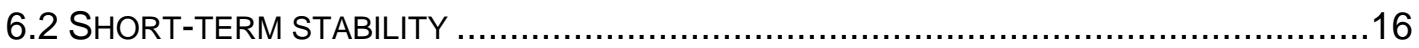

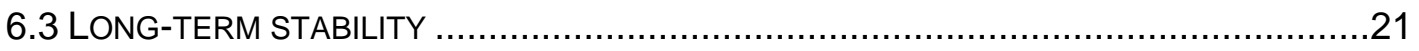

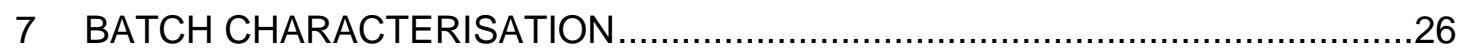

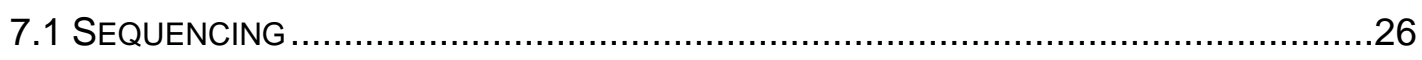

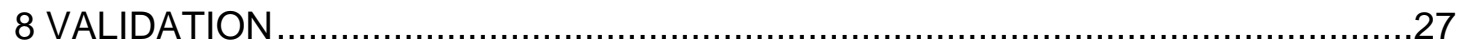

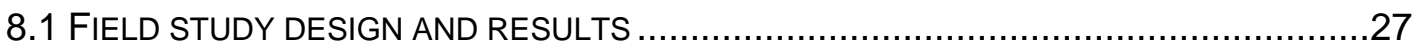

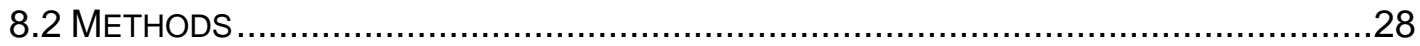

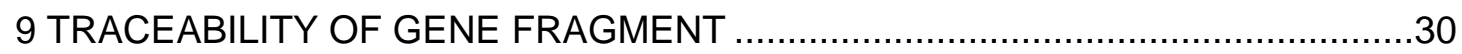

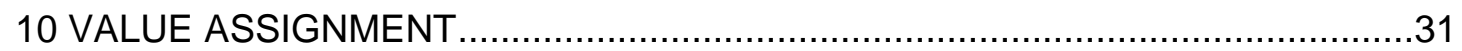

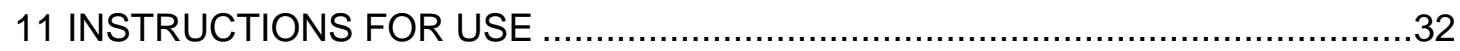

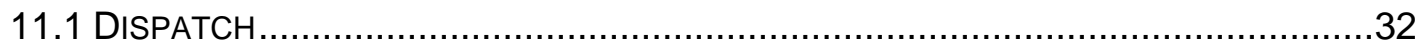

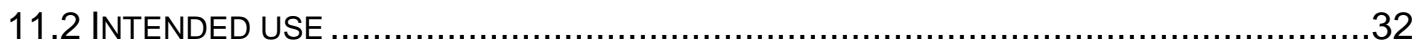

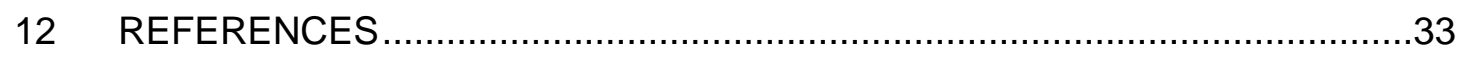




\section{GLOSSARY}

\begin{tabular}{|c|c|}
\hline A & Adenine \\
\hline ANOVA & analysis of variance \\
\hline & slope of the regression line \\
\hline bp & base pairs \\
\hline C & Cytosine \\
\hline Cp & $\begin{array}{l}\text { Crossing point. The } \mathrm{Cp} \text { value is defined as the crossing point } \\
\text { threshold and defines the threshold above which an amplification } \\
\text { signal can be detected. }\end{array}$ \\
\hline CRM & Certified Reference Material \\
\hline $\mathrm{Ct}$ & $\begin{array}{l}\text { Cycle threshold. The Ct value is defined as the cycle threshold and } \\
\text { defines the threshold above which an amplification signal can be } \\
\text { detected using real-time PCR. }\end{array}$ \\
\hline DNA & deoxyribo nucleic acid \\
\hline DTCS & Dye Terminator Cycle Sequencing \\
\hline$\Delta \mathrm{T}_{\mathrm{m}}$ & difference in melting temperature \\
\hline FAM & 6-carboxy fluorescein \\
\hline FRET & Fluorescence Resonance Energy Transfer \\
\hline & Guanine \\
\hline G20210A & $\begin{array}{l}\text { mutation at position } 20210 \text { resulting from the replacement of a } \\
\text { Guanine by an Adenine in the sequence }\end{array}$ \\
\hline IFCC & $\begin{array}{l}\text { International Federation of Clinical Chemistry and Laboratory } \\
\text { Medicine }\end{array}$ \\
\hline IRMM & Institute for Reference Materials and Measurements \\
\hline$M S_{\text {among }}$ & mean of squares among vials (from ANOVA) \\
\hline$M S_{\text {within }}$ & mean of squares within vials (from ANOVA) \\
\hline$N$ & total number of vials in the homogeneity study \\
\hline $\mathrm{p}$ & probability \\
\hline PCR & polymerase chain reaction \\
\hline pDNA & plasmid deoxyribo nucleic acid \\
\hline RFLP & Restriction Fragment Length Polymorphism \\
\hline$s_{\mathrm{bb}}$ & standard deviation between the vials \\
\hline$s$ & absolute standard deviation \\
\hline SS & the sum of the squares of the data \\
\hline$t_{\alpha, v}$ & $\begin{array}{l}t \text {-value (two-sided) for a confidence level } \alpha \text { and } v \text { degrees of } \\
\text { freedom (from regression analysis) }\end{array}$ \\
\hline$t$ & time \\
\hline $\mathrm{T}$ & Thymine \\
\hline TAMRA & 6-carboxy-tetra-methyl-rhodamine \\
\hline TE & $10 \mathrm{mmol} / \mathrm{L}$ Tris, $1 \mathrm{mmol} / \mathrm{L}$ EDTA, $\mathrm{pH} 8.0$ \\
\hline$T_{m}$ & $\begin{array}{l}\text { melting temperature. The melting temperature is the temperature at } \\
\text { which } 50 \% \text { of the oligonucleotides are in double-stranded } \\
\text { conformation and } 50 \% \text { are single stranded }\end{array}$ \\
\hline$u_{\mathrm{b}}$ & standard uncertainty of the slope of the regression line \\
\hline$u_{b b}{ }^{*}$ & $\begin{array}{l}\text { standard uncertainty due to inhomogeneity that can be hidden by } \\
\text { method repeatability. It is used as the minimum uncertainty } \\
\text { contribution from inhomogeneity between vials. }\end{array}$ \\
\hline$v$ & degrees of freedom \\
\hline
\end{tabular}




\section{Introduction}

Molecular genetic tests are used in medical diagnostics to identify changes in DNA sequences to diagnose or predict an inherited disorder. These tests should be highly selective. The use of an adequate reference material to validate test equipment and measurement procedures is an important part of any quality control system. Currently, only few CRMs are available for molecular genetic testing.

Thus, in collaboration with the International Federation of Clinical Chemistry and Laboratory Medicine (IFCC), a DNA reference material was produced for the detection of a single point mutation (G20210A) in the gene coding for the human prothrombin (Factor II). This gene mutation has been identified as one of the risk factors in venous thrombosis events.

Currently, several measurement procedures are used among laboratories to detect the G20210A mutation in the prothrombin gene. All of them are based on a PCR amplification followed either by restriction enzyme digestion, hybridisation protocols, single-strand conformation polymorphism analysis, LightCycler PCR analysis, denaturating gradient gel analysis or sequencing.

Therefore, a DNA fragment that spans the area of the human prothrombin gene containing the G20210A mutation has been used to produce the reference material. This specific region can be amplified with all primer combinations described in the literature for prothrombin mutation analysis.

Besides the mutated DNA fragment, the wildtype DNA fragment was produced as well. Both fragments were cloned separately into the pUC18 plasmid. The concentration per vial was adjusted to $1 \mathrm{ng}$ per $50 \mu \mathrm{L}$.

For the present CRM IRMM/IFCC-492, a batch of 1500 vials of purified pDNA containing equal volumes of identical mass concentrations of the wildtype and the mutant (G20210A) sequence of a cloned $609 \mathrm{bp}$ fragment for the human prothrombin gene (i.e. mix of equal volumes of identical mass concentrations of IRMM/IFCC-490 and IRMM/IFCC-491) was produced. The certification of IRMM/IFCC-492 was performed according to IRMM Reference Materials Unit procedures applying the ISO Guide 34 quality management system [1-4]. Stability measurements were performed at different temperatures $\left(-20^{\circ} \mathrm{C}, 4{ }^{\circ} \mathrm{C}, 18^{\circ} \mathrm{C}\right.$ and $\left.40{ }^{\circ} \mathrm{C}\right)$. Sequence identity in the production batch before dilution was assessed by DNA sequence analysis of the fragments inserted into the pUC18 vectors.

The produced batch was characterised for homogeneity.

Samples in short-term stability measurements were analysed by melting curve experiments using PCR and the commercial LightCycler kit for Factor II (prothrombin) 
G20210A mutation detection [5]. Samples in long-term stability measurements were analysed by real-time PCR experiments using specific primers and probe. 


\section{Participants (Table 1)}

The following laboratories with expertise in genetic testing were involved in the field study:

Table 1: List of participants for the characterisation of the material

\begin{tabular}{|l|l|l|}
\hline & $\begin{array}{l}\text { Institut für Medizinische und Chemische } \\
\text { Labordiagnostik mit Thromboseambulanz / } \\
\text { Kalbmayer, W. }\end{array}$ & AT-1130 Wien \\
\hline Zadro, R. & $\begin{array}{l}\text { Clinical Institute of Laboratory Diagnosis / Clinical } \\
\text { Hospital Center Zagreb University School of } \\
\text { Medicine }\end{array}$ & HR-10000 Zagreb \\
\hline Melegh, B. & $\begin{array}{l}\text { University of Pecs, Department of Medical } \\
\text { Genetics and Child Development }\end{array}$ & HU-7624 Pecs \\
\hline Gemmati, D. & $\begin{array}{l}\text { Ctr. Study Haemostasis and Thrombosis, } \\
\text { University of Ferrara }\end{array}$ & IT-44100 Ferrara \\
\hline Spannagl, M. & $\begin{array}{l}\text { Ludwig-Maximilians-Universität, Klinikum } \\
\text { Innenstadt, Hämostaseologische Ambulanz }\end{array}$ & DE-80336 München \\
\hline Kitchen, S. & $\begin{array}{l}\text { Dept. of Coagulation / Sheffield Haemophilia and } \\
\text { Thrombosis Centre / Royal Hallamshire Hospital }\end{array}$ & GB- S10 2 JF Sheffield \\
\hline Mannhalter, C. & $\begin{array}{l}\text { Klinisches Institut für Medizinische und } \\
\text { Chemische Labordiagnostik (KIMCL) }\end{array}$ & AT-1090 Wien \\
\hline
\end{tabular}




\section{Processing steps}

The production and certification of IRMM/IFCC-492 was performed by IRMM by mixing equal volumes of identical mass concentrations of IRMM/IFCC-490 and IRMM/IFCC-491.

The processing steps for the production of IRMM/IFCC-490 and IRMM/IFCC-491 are described in Klein et al, [6] and in their respective certification reports.

The concentration of the plasmids was measured by double-stranded DNA PicoGreen quantitation method [7]. The pDNA stock concentration and standard deviation of IRMM/IFCC-490 was $1390 \pm 29 \mu \mathrm{g} / \mathrm{mL}$. The pDNA stock concentration and standard deviation of IRMM/IFCC-491 was $1823 \pm 29 \mu \mathrm{g} / \mathrm{mL}$.

The plasmid stock solutions were diluted in TE pH 8.0 to reach, for each pDNA, a final concentration of $0.02 \mathrm{ng} \mathrm{pDNA} / \mu \mathrm{L}$ or $1 \mathrm{ng} / 50 \mu \mathrm{L}$. Then equal volumes of these plasmid solutions were mixed and the resulting solution was aliquoted, the total amount of pDNA being $1 \mathrm{ng} / \mu \mathrm{L}$ (0.5 ng/ $\mu \mathrm{L}$ IRMM/IFCC-490 and $0.5 \mathrm{ng} / \mu \mathrm{L}$ IRMM/IFCC-491).

One thousand five hundred polypropylene vials (sterile, $1.5 \mathrm{~mL}$ volume) were filled manually with $50 \mu \mathrm{L}$ of pDNA solution (mass concentration $0.02 \mathrm{ng} / \mu \mathrm{L}$ TE buffer $\mathrm{pH}$ 8.0).

The vials were closed with screw caps in a laminar flowhood and labelled. Homogeneity and stability studies as well as batch characterisation were carried out. 


\section{Procedures}

\section{Minimum sample volume}

The minimum sample volume was $5 \mu \mathrm{L}$ as recommended in the protocol of the Factor II (prothrombin) G20210A kit used in combination with the LightCycler. Dilution experiments during the short-term stability study gave an indication of the limit of detection.

\section{Quantification of pDNA}

Quantification of double-stranded pDNA in stock solution was performed with PicoGreen reagent in a microtiter plate using fluorimetry (excitation at $480 \mathrm{~nm}$ and emission at $525 \mathrm{~nm}$ ) [7]. A standard DNA calibration curve was prepared with lambda phage DNA at mass concentrations ranging from 0 to $1 \mathrm{ng} / \mu \mathrm{L}$. The test was prepared in a microtiter plate, and fluorescence was detected in a FLUOstar Galaxy plate reader (BMG LABTECH GmbH, Offenburg, Germany) [8].

\section{Quantitative real-time PCR}

The principle of the real-time quantitative PCR is the use of a specific fluorescent probe in addition to the primers. The fluorescence intensity is proportional to the number of target DNA sequences. This fluorescence is recorded on-line during the amplification process. The TaqMan ${ }^{\circledR}$ probe contains a reporter dye (FAM) at its $5^{\prime}$ end and a quencher (TAMRA) dye at its 3 ' end.

Accumulation of PCR products is detected directly by monitoring the increase in fluorescence of the reporter dye. The number of cycles which are required to pass a fluorescence threshold (Ct-value) correlates with the amount of target DNA in the original sample.

We used the ABI Prism 7700 and ABI Prism 7900HT device (Applied Biosystems, Lennik, Belgium).

Each reaction mixture for real-time PCR reactions ( $25 \mu \mathrm{L}$ total volume) contained $12.5 \mu \mathrm{L}$ of 2-times Universal PCR Master Mix (Applied Biosystems, Lennik, Belgium), $2.5 \mu \mathrm{L}$ of forward primer $(9 \mu \mathrm{mol} / \mathrm{L}), 2.5 \mu \mathrm{L}$ of reverse primer $(9 \mu \mathrm{mol} / \mathrm{L}), 2.5 \mu \mathrm{L}$ of probe $(2 \mu \mathrm{mol} / \mathrm{L})$ and $5 \mu \mathrm{L}$ pDNA template. The sequences of the PCR primers and probe (Applied Biosystems, Lennik, Belgium) used are summarized in Table 2.

PCR reactions were performed using the following cycling programme: 2 min at 50 ${ }^{\circ} \mathrm{C}, 10 \mathrm{~min}$ at $95^{\circ} \mathrm{C},\left(15 \mathrm{~s}\right.$ at $95^{\circ} \mathrm{C}, 1 \mathrm{~min}$ at $\left.60^{\circ} \mathrm{C}\right)$ for 30 cycles. Ct values were obtained using the SDS 2.2.2 software on ABI Prism 7900 HT and SDS 1.9.1 on ABI Prism 7700 instrument.

Each sample was analysed in triplicate. Triplicates were randomized on the 96-wells plates, and an internal control (pDNA at $0.02 \mathrm{ng} / \mu \mathrm{L}$ ) was displayed on each plate to allow comparison of data on the different instruments. 
Table 2. Primers and probe used for the quantitative PCR

\begin{tabular}{|l|l|l|}
\hline & $\begin{array}{l}\text { IRMM Primer } \\
\text { Code number }\end{array}$ & Sequence \\
\hline Forward & P-0564 & 5'-GCTCACCGGCTCCAGATTT -3' \\
\hline Reverse & P-0565 & 5'- TTCTGCGCTCGGCCC-3' \\
\hline Probe & P-0566 & FAM-5'-TCAGCAATAAACCAGCCAGCCGGA-3'-TAMRA \\
\hline
\end{tabular}

\section{Genotyping using the LightCycler technology}

The LightCycler Factor II (prothrombin) G20210A kit (Roche, cat \#036 10195001, [5]) provides primers and hybridisation probes for sequence-specific detection and includes a control template heterozygous for the G20210A mutation for reliable interpretation, as well as a negative control consisting of DNA-free water.

Principle: combining hybridisation probe detection with subsequent melting curve analysis makes applications such as rapid genotyping and mutation analysis possible (example in Figure 1A and 1B). Indeed, a single mismatch between the hybridisation probe and its target sequence, caused by a single point mutation, changes the melting temperature of the bound probe. Therefore, the recording of the melting temperature $\left(T_{m}\right)$ in the plot of the negative first derivative of the fluorescence intensity versus temperature $(-\mathrm{dF} / \mathrm{dT})$ indicates the genotype of the PCR product (Figure 1B).

Quantitative data on the DNA used as template in the PCR reaction is given by the Crossing point (Cp) threshold obtained by "automatic call" in the programme. The $\mathrm{Cp}$ value is defined as the crossing point threshold and defines the threshold above which an amplification signal can be detected. 


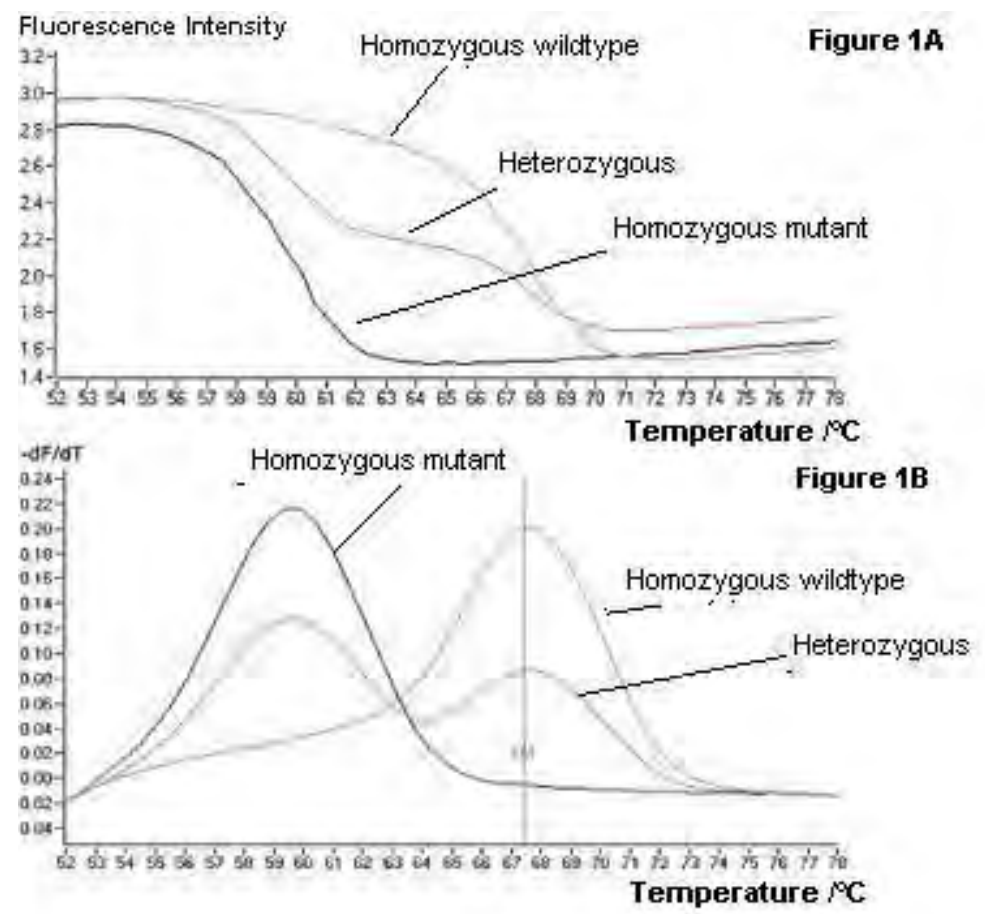

Figure 1: Example of analysis and interpretation of data using the LightCycler technology and the melting curve analysis

The position of the peak(s) of the melting curve obtained with the components of the kit allow a discrimination between the homozygous (wildtype or mutant) as well as the heterozygous genotypes. The $T_{m}$ does not only depend on the length and $G+C$ content, but also depends on the degree of homology between the mutation probe and the template DNA. In the LightCycler Factor II (prothrombin) G20210A kit, the wildtype genotype and the fluorescein-labeled hybridization probe form a perfect match resulting in a $T_{m}$ of about $59{ }^{\circ} \mathrm{C}$. The mutant genotype and the fluoresceinlabeled hybridization probe form a mismatch, resulting in a $T_{m}$ of about $49{ }^{\circ} \mathrm{C}$. The kit manual stipulates that the $\mathrm{T}_{\mathrm{m}}$ values may vary for $\pm 2.5{ }^{\circ} \mathrm{C}$ between different experiments, and that the $\Delta \mathrm{T}_{\mathrm{m}}$ should be equal to $(10 \pm 1.5){ }^{\circ} \mathrm{C}$.

\section{DNA sequencing}

Between 100 and 200 ng of pDNA were used in Dye Terminator Cycle Sequencing (DTCS) reaction, according to the DTCS Chemistry Protocol [9] and using the primers P-0289, P-0419, P-0579 and P-0580 (Table 3). The following thermal cycling programme was used: 30 cycles of $96{ }^{\circ} \mathrm{C}$ for $20 \mathrm{~s}, 50{ }^{\circ} \mathrm{C}$ for $20 \mathrm{~s}, 60^{\circ} \mathrm{C}$ for $4 \mathrm{~min}$; and hold at $4{ }^{\circ} \mathrm{C}$. Samples were analysed on a CEQ ${ }^{\text {TM }} 8000$ Genetic Analysis System (Beckman Coulter, Inc., Fullerton, CA, USA), using the following method: denaturation $120 \mathrm{~s}$ at $90{ }^{\circ} \mathrm{C}$, injection $15 \mathrm{~s}$ at $2.0 \mathrm{kV}$, separation $85.0 \mathrm{~min}$ at $4.2 \mathrm{kV}$. 
Table 3: Primers used for Factor II sequencing

\begin{tabular}{|l|l|l|}
\hline & $\begin{array}{l}\text { IRMM Primer } \\
\text { Code number }\end{array}$ & Sequence \\
\hline Forward (M13-F) & P-0419 & 5'- GTA AAA CGA CGG CCA G-3' \\
\hline Reverse (M13-R) & P-0289 & 5'- CAG GAA ACA GCT ATG AC-3' \\
\hline Forward (Factor II-F) & P-0579 & 5'- TAC TGA TGT GAC CTT GAA CT-3' \\
\hline Reverse (Factor II-R) & P-0580 & 5'- CTT CTG TAT CCA CTT CTT CA-3' \\
\hline
\end{tabular}




\section{Homogeneity}

\subsection{Planning of homogeneity study}

1500 vials were produced. The minimum required number of vials used in the homogeneity study $(N)$ is calculated as ${ }^{3} \sqrt{1500}=12$ [1-2]. The homogeneity study was performed using data from samples kept at $-70{ }^{\circ} \mathrm{C}(N=6)$ and at $-20{ }^{\circ} \mathrm{C}(N=18)$ in the short-term stability study. Two replicates of $5 \mu \mathrm{L}$ of IRMM/IFCC-492 were analysed using the LightCycler prothrombin G20210A mutation detection kit and the Cp values were assessed.

The average of the difference in melting temperature $\left(\Delta \mathrm{T}_{\mathrm{m}}\right)$ between the wildtype and the mutant products was recorded additionally as it confirmed the identity of the sequence (using defined primers and probes). This difference $\pm s$ should be equal to $(10 \pm 1.5)^{\circ} \mathrm{C}$, according to the kit specifications.

\subsection{Evaluation of homogeneity study}

The results of the Crossing point threshold $(\mathrm{Cp})$ and average $\Delta \mathrm{T}_{\mathrm{m}}$ measurements are summarized in Table 4. The positive control was a fragment of human DNA, heterozygous for the mutation, and the negative control was DNA-free water (both are included in the kit). 
Table 4. Homogeneity of the $\mathrm{Cp}$ and $\Delta \mathrm{T}_{\mathrm{m}}$ values of IRMM/IFCC-492. Vials were stored at $-70^{\circ} \mathrm{C}$ or at $-20^{\circ} \mathrm{C}$. Suspicious data are in bold.

\begin{tabular}{|c|c|c|c|c|c|c|c|}
\hline \multicolumn{8}{|c|}{ Homogeneity of $\mathrm{Cp}$ and $\Delta \mathrm{T}_{\mathrm{m}}$ values after storage at $-70^{\circ} \mathrm{C}$ or $-20^{\circ} \mathrm{C}$ for 2,4 or 8 weeks } \\
\hline $\begin{array}{c}\text { Vial } \\
\text { Number }\end{array}$ & $\begin{array}{l}\text { Storage time at } \\
-70^{\circ} \mathrm{C} \text { (weeks) }\end{array}$ & $\begin{array}{c}\text { Replicate } \\
1\end{array}$ & $\begin{array}{c}\text { Replicate } \\
2\end{array}$ & $\begin{array}{c}\text { Average } \\
\text { Cp }\end{array}$ & $s$ Cp & $\begin{array}{c}\text { Average } \\
\Delta \mathrm{T}_{\mathrm{m}}\end{array}$ & $\mathrm{s} \Delta \mathrm{T}_{\mathrm{m}}$ \\
\hline 9 & 8 & 15.29 & 15.11 & 15.20 & 0.13 & 9.54 & 0.08 \\
\hline 232 & 8 & 15.67 & 15.05 & 15.36 & 0.44 & 9.58 & 0.07 \\
\hline 469 & 8 & 17.27 & 15.20 & 16.24 & 1.46 & 9.45 & 0.05 \\
\hline 706 & 8 & 15.04 & 15.09 & 15.07 & 0.04 & 9.51 & 0.02 \\
\hline 945 & 8 & 15.01 & 14.97 & 14.99 & 0.03 & 9.52 & 0.11 \\
\hline 1166 & 8 & 14.76 & 15.05 & 14.91 & 0.21 & 9.44 & 0.17 \\
\hline $\begin{array}{c}\text { Vial } \\
\text { Number }\end{array}$ & $\begin{array}{l}\text { Storage time at } \\
-20^{\circ} \mathrm{C} \text { (weeks) }\end{array}$ & $\begin{array}{c}\text { Replicate } \\
1\end{array}$ & $\begin{array}{c}\text { Replicate } \\
2\end{array}$ & $\begin{array}{c}\text { Average } \\
C p\end{array}$ & $s C p$ & $\begin{array}{c}\text { Average } \\
\Delta \mathbf{T}_{\mathrm{m}}\end{array}$ & $s \Delta T_{m}$ \\
\hline 81 & 2 & 14.85 & 15.13 & 14.99 & 0.20 & 9.54 & 0.06 \\
\hline 310 & 2 & 15.81 & 15.63 & 15.72 & 0.13 & 9.54 & 0.04 \\
\hline 550 & 2 & 15.67 & 15.15 & 15.41 & 0.37 & 9.53 & 0.05 \\
\hline 775 & 2 & 15.00 & 14.91 & 14.96 & 0.06 & 9.54 & 0.04 \\
\hline 1024 & 2 & 14.99 & 15.05 & 15.02 & 0.04 & 9.47 & 0.06 \\
\hline 1250 & 2 & 15.31 & 15.05 & 15.18 & 0.18 & 9.44 & 0.04 \\
\hline 85 & 4 & 14.99 & 15.21 & 15.10 & 0.16 & 9.56 & 0.08 \\
\hline 329 & 4 & 15.12 & 15.76 & 15.44 & 0.45 & 9.58 & 0.12 \\
\hline 560 & 4 & 15.47 & 15.27 & 15.37 & 0.14 & 9.49 & 0.03 \\
\hline 796 & 4 & 14.87 & 15.55 & 15.21 & 0.48 & 9.57 & 0.04 \\
\hline 1029 & 4 & 14.97 & 15.28 & 15.13 & 0.22 & 9.42 & 0.09 \\
\hline 1268 & 4 & 14.95 & 15.67 & 15.31 & 0.51 & 9.56 & 0.05 \\
\hline 91 & 8 & 14.84 & 14.88 & 14.86 & 0.03 & 9.33 & 0.04 \\
\hline 337 & 8 & 14.75 & 14.95 & 14.85 & 0.14 & 9.45 & 0.08 \\
\hline 584 & 8 & 14.86 & 14.81 & 14.84 & 0.04 & 9.37 & 0.08 \\
\hline 808 & 8 & 14.96 & 14.91 & 14.94 & 0.04 & 9.30 & 0.04 \\
\hline 1050 & 8 & 14.83 & 14.89 & 14.86 & 0.04 & 9.30 & 0.01 \\
\hline 1270 & 8 & 14.85 & 14.92 & 14.89 & 0.05 & 9.29 & 0.03 \\
\hline
\end{tabular}

In Table 4, the grand average and standard deviation of $\mathrm{Cp}$ is $15.16 \pm 0.32$ and of $\Delta \mathrm{T}_{\mathrm{m}}=(9.47 \pm 0.09){ }^{\circ} \mathrm{C}$.

Scrutiny of the data: one suspicious datum was detected (in bold), and a trend was observed when it was not removed from the data set ( $t$-test; $t_{0.05,23}$, data not shown). The outlying datum was however retained in the evaluation, because here no distinction could be made between the influence of statistical variation and inhomogeneity of the batch.

All average $\Delta T_{m}$ values $\pm s$ of the individual samples (Table 4 ) are within the expected interval mentioned in the kit manual $(10 \pm 1.5){ }^{\circ} \mathrm{C}$. Therefore, all samples could be categorised as having the heterozygous genotype. Moreover, since the batch has been prepared by dilution from a stock solution that had been sequenced and since all samples are liquid (frozen at $-20^{\circ} \mathrm{C}$ ), heterogeneity is unlikely to be found.

Analysis of the data by ANOVA on all $\mathrm{Cp}$ values is summarized in Table 5 (using MS Excel $^{\mathrm{TM}}$ Single Factor ANOVA). 
Table 5. Analysis of data from Table 4 using MS Excel ${ }^{\mathrm{TM}}$ Single Factor ANOVA

\begin{tabular}{|l|r|r|r|}
\hline & SS & v & MS \\
\hline Among vials & 4.855 & 23 & 0.211 \\
\hline Within vials & 3.440 & 24 & 0.143 \\
\hline Total & 8.295 & 47 & \\
\hline
\end{tabular}

From Table 5 the repeatability of the procedure was calculated as $2.50 \%$, according to the procedures described in references [1-4]. The $s_{\mathrm{bb}}$ was calculated as $4.73 \%$, and the relative standard uncertainty $u_{b b}{ }^{*}$ as $0.95 \%$. The maximum relative standard uncertainty contribution to homogeneity was therefore $4.73 \%$.

A plot of the $\mathrm{Cp}$ values of the PCR products versus the sample number did not reveal a trend in the filling sequence (data not shown). The slope of the regression line was not significantly different from zero (t-test, data not shown) and the data followed a normal and unimodal distribution (data not shown).

In conclusion, the batch IRMM/IFCC-492 produced is homogeneous and contains the heterozygous genotype G20210 wildtype/G20210A mutant for the sequence of a 609 bp fragment of the human prothrombin gene. 


\section{Stability}

\subsection{Planning}

The stability studies have been assessed by isochronous measurements at time points of 2, 4 and 8 weeks for the short-term, and at 2, 4 and 6 months for the longterm study. The reference temperature was set at $-70^{\circ} \mathrm{C}$, corresponding to time point 0 .

\subsection{Short-term stability}

In order to assess the stability of the material, an indication of the pDNA quantity in each vial was investigated using the $\mathrm{Cp}$ values obtained after the PCR reaction in the LightCycler. Six different vials were tested in duplicate ( $5 \mu \mathrm{L}$ pDNA per PCR reaction) at each temperature. As the $C p$ values give an indication of the quantity of the pDNA remaining after storage, the melting temperatures allow genotyping of the DNA fragments (Tables 6.1 and 6.2).

Table 6.1: Short-term stability of IRMM/IFCC-492 stored for 8 weeks at $-70^{\circ} \mathrm{C}$.

Suspicious data are in bold

\begin{tabular}{|l|c|c|c|c|}
\hline & \multicolumn{2}{|c|}{ Cp values } & \multicolumn{2}{c|}{$\Delta \mathbf{T}_{\mathbf{m}}$ values } \\
\hline & $\mathbf{C p ~ 1}$ & $\mathbf{C p ~ 2}$ & $\Delta \mathbf{T}_{\mathbf{m}} \mathbf{1}$ & $\Delta \mathbf{T}_{\mathbf{m}} \mathbf{2}$ \\
\hline Sample 1 & 15.20 & 14.38 & 9.54 & 9.50 \\
\hline Sample 2 & 15.36 & 14.50 & 9.58 & 9.45 \\
\hline Sample 3 & $\mathbf{1 6 . 2 4}$ & 14.52 & 9.45 & 9.40 \\
\hline Sample 4 & 15.07 & 14.36 & 9.51 & 9.41 \\
\hline Sample 5 & 14.99 & 14.51 & 9.52 & 9.46 \\
\hline Sample 6 & 14.91 & 14.55 & 9.44 & 9.40 \\
\hline Average & 15.29 & 14.47 & 9.51 & 9.44 \\
\hline S & 0.49 & 0.08 & 0.05 & 0.04 \\
\hline
\end{tabular}

Cp 1 and $\Delta T_{m} 1$ represent respectively the averages of $\mathrm{Cp}$ and $\Delta \mathrm{T}_{\mathrm{m}}$ values of a duplicate measurement of samples stored at the reference temperature in the isochronous study investigating the stability at $-20^{\circ} \mathrm{C}, 4{ }^{\circ} \mathrm{C}$ and $18^{\circ} \mathrm{C}$. $\mathrm{Cp} 2$ and $\Delta \mathrm{T}_{\mathrm{m}}$ 2 represent respectively the averages of $\mathrm{Cp}$ and $\Delta \mathrm{T}_{\mathrm{m}}$ values of a duplicate measurement of samples stored at the reference temperature in the isochronous study investigating the stability at $40{ }^{\circ} \mathrm{C}$. The average and standard deviation of the $\mathrm{Cp}$ values and $\Delta \mathrm{T}_{\mathrm{m}}$ values of samples stored at $-70^{\circ} \mathrm{C}$ is respectively $\mathrm{Cp} 1=15.29 \pm$ $0.49, \mathrm{Cp} 2=14.47 \pm 0.08$ and $\Delta \mathrm{T}_{\mathrm{m}} 1=(9.51 \pm 0.05){ }^{\circ} \mathrm{C}, \Delta \mathrm{T}_{\mathrm{m}} 2=(9.44 \pm 0.04)^{\circ} \mathrm{C}$. 
The samples of Table 6.1 were analysed simultaneously with those in Table 6.2 as they represented the samples stored at the reference temperature in the stability study. Scrutiny of the data: one suspicious datum (indicated in bold) was detected with a discrepancy of more than $50 \%$ to the average between vials at the corresponding time point and temperature. The outlying data at $-70{ }^{\circ} \mathrm{C}$ was considered in the evaluation, because here no distinction can be made between the influence of statistical variation and inhomogeneity of the batch. 
Table 6.2: Short-term stability of IRMM/IFCC-492 stored for 2, 4 and 8 weeks at $-20^{\circ} \mathrm{C}, 4^{\circ} \mathrm{C}, 18^{\circ} \mathrm{C}$ or $40^{\circ} \mathrm{C}$. Suspicious data are in bold

\begin{tabular}{|c|c|c|c|c|}
\hline & & \multicolumn{3}{|c|}{ Cp values at $t$ (week) } \\
\hline & $\begin{array}{l}\text { Temperature } \\
{ }^{\circ} \mathrm{C}\end{array}$ & $t=2$ & $t=4$ & $t=8$ \\
\hline Sample 1 & -20 & 14.99 & 15.10 & 14.86 \\
\hline Sample 2 & & 15.72 & 15.44 & 14.85 \\
\hline Sample 3 & & 15.41 & 15.37 & 14.84 \\
\hline Sample 4 & & 14.96 & 15.21 & 14.94 \\
\hline Sample 5 & & 15.02 & 15.13 & 14.86 \\
\hline Sample 6 & & 15.18 & 15.31 & 14.89 \\
\hline Average & & 15.21 & 15.26 & 14.87 \\
\hline$s$ & & 0.30 & 0.14 & 0.04 \\
\hline Sample 1 & 4 & 14.98 & 14.92 & 14.77 \\
\hline Sample 2 & & 15.23 & 14.90 & 14.86 \\
\hline Sample 3 & & 14.62 & 14.92 & 14.79 \\
\hline Sample 4 & & 15.13 & 14.90 & 14.92 \\
\hline Sample 5 & & 15.14 & 14.76 & 14.76 \\
\hline Sample 6 & & 15.00 & 14.93 & 14.74 \\
\hline Average & & 15.01 & 14.89 & 14.81 \\
\hline$s$ & & 0.22 & 0.06 & 0.07 \\
\hline Sample 1 & 18 & 14.17 & 13.65 & 12.73 \\
\hline Sample 2 & & 14.34 & 13.64 & 12.86 \\
\hline Sample 3 & & 14.28 & 13.71 & 12.85 \\
\hline Sample 4 & & 14.44 & 13.55 & 13.00 \\
\hline Sample 5 & & 13.82 & 13.34 & 12.95 \\
\hline Sample 6 & & 14.19 & 13.44 & 11.61 \\
\hline Average & & 14.20 & 13.55 & 12.66 \\
\hline$s$ & & 0.22 & 0.14 & 0.53 \\
\hline Sample 1 & 40 & 9.52 & 10.78 & 9.02 \\
\hline Sample 2 & & 9.49 & 10.35 & 10.91 \\
\hline Sample 3 & & 10.77 & 10.46 & 13.88 \\
\hline Sample 4 & & 7.65 & 9.30 & 8.37 \\
\hline Sample 5 & & 10.57 & 9.47 & 9.61 \\
\hline Sample 6 & & 9.42 & 10.52 & 11.20 \\
\hline Average & & 9.57 & 10.15 & 10.50 \\
\hline$s$ & & 1.11 & 0.61 & 1.98 \\
\hline
\end{tabular}


The grand average and standard deviation of the $\mathrm{Cp}$ values of the vials stored at -20 ${ }^{\circ} \mathrm{C}$ is $\mathrm{Cp}=15.11 \pm 0.25, \mathrm{Cp}=14.90 \pm 0.16$ at $4{ }^{\circ} \mathrm{C}, \mathrm{Cp}=13.47 \pm 0.72$ at $18{ }^{\circ} \mathrm{C}$ and $\mathrm{Cp}=10.07 \pm 1.33$ at $40^{\circ} \mathrm{C}$ (Table 6.2 ). Scrutiny of the data: one suspicious datum (indicated in bold) was detected with a discrepancy of more than $50 \%$ to the average between vials at the corresponding time point and temperature. The outlying data at $40{ }^{\circ} \mathrm{C}$ was considered in the evaluation, because here no distinction can be made between the influence of statistical variation and inhomogeneity of the batch.

For the four temperatures the slope of the regression line and its uncertainty were calculated and tested for statistical significance (Figure 2).

At all temperatures, the slope of the regression line was significantly different from 0 , even when removing the suspicious datum ( $t$-test; $t_{0.05,23}$, data not shown), but as the concentration of the material was slightly increasing with time (i.e. decreasing $\mathrm{Cp}$ values), the regression line was technically insignificant.

The recovery of the material was increasing, especially after storage at $40{ }^{\circ} \mathrm{C}$, due to evaporation of the solution with storage time. However, the reconstitution of the dried material (after 8 weeks at $40^{\circ} \mathrm{C}$ ) in a $50 \mu \mathrm{L}$ volume of water showed that the pDNA sequence was still present in sufficient amount to allow correct genotyping (Table 7).
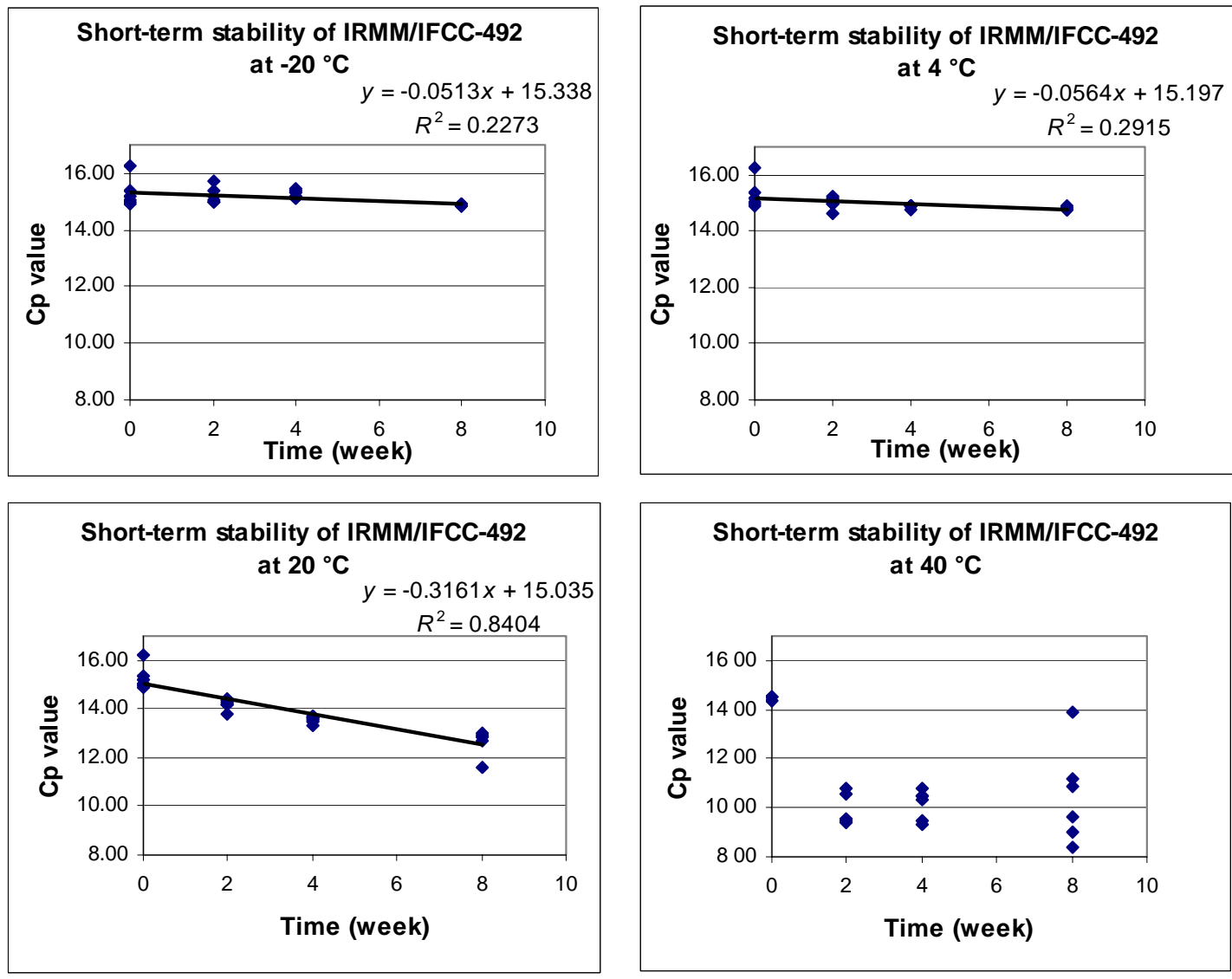

Short-term stability of IRMM/IFCC-492 at $40{ }^{\circ} \mathrm{C}$

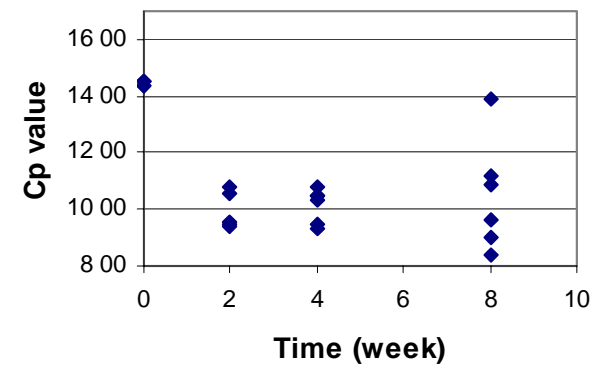

Figure 2. Slopes of the regression lines of IRMM/IFCC-492 during short-term stability study $\left(0,2,4\right.$ and 8 weeks at $-20^{\circ} \mathrm{C}, 4^{\circ} \mathrm{C}, 18^{\circ} \mathrm{C}$ and $\left.40^{\circ} \mathrm{C}\right)$ 
Table 7: Difference in melting temperature $I^{\circ} \mathrm{C}$ of short-term stability samples of IRMM/IFCC- 492 stored for 2,4 and 8 weeks at $-20^{\circ} \mathrm{C}, 4^{\circ} \mathrm{C}, 18^{\circ} \mathrm{C}$ or $40^{\circ} \mathrm{C}$

\begin{tabular}{|c|c|c|c|c|}
\hline & \multicolumn{4}{|c|}{$\Delta \mathbf{T}_{\mathrm{m}}$ values at $\mathrm{t}$ (week) } \\
\hline & $\begin{array}{l}\text { Temperature } \\
{ }^{\circ} \mathrm{C}\end{array}$ & $t=2$ & $t=4$ & $t=8$ \\
\hline Sample 1 & -20 & 9.54 & 9.56 & 9.33 \\
\hline Sample 2 & & 9.54 & 9.58 & 9.45 \\
\hline Sample 3 & & 9.53 & 9.49 & 9.37 \\
\hline Sample 4 & & 9.54 & 9.57 & 9.30 \\
\hline Sample 5 & & 9.47 & 9.42 & 9.30 \\
\hline Sample 6 & & 9.44 & 9.56 & 9.29 \\
\hline Average & & 9.51 & 9.53 & 9.34 \\
\hline$s$ & & 0.04 & 0.06 & 0.06 \\
\hline Sample 1 & 4 & 9.54 & 9.43 & 9.25 \\
\hline Sample 2 & & 9.57 & 9.50 & 9.23 \\
\hline Sample 3 & & 9.49 & 9.50 & 9.23 \\
\hline Sample 4 & & 9.40 & 9.42 & 9.24 \\
\hline Sample 5 & & 9.55 & 9.44 & 9.22 \\
\hline Sample 6 & & 9.47 & 9.43 & 9.25 \\
\hline Average & & 9.50 & 9.45 & 9.23 \\
\hline$s$ & & 0.06 & 0.04 & 0.01 \\
\hline Sample 1 & 18 & 9.56 & 9.65 & 9.43 \\
\hline Sample 2 & & 9.61 & 9.53 & 9.44 \\
\hline Sample 3 & & 9.58 & 9.50 & 9.42 \\
\hline Sample 4 & & 9.59 & 9.55 & 9.36 \\
\hline Sample 5 & & 9.53 & 9.46 & 9.40 \\
\hline Sample 6 & & 9.53 & 9.53 & 9.42 \\
\hline Average & & 9.56 & 9.54 & 9.41 \\
\hline$s$ & & 0.03 & 0.06 & 0.03 \\
\hline Sample 1 & 40 & 9.51 & 9.47 & 9.44 \\
\hline Sample 2 & & 9.51 & 9.46 & 9.38 \\
\hline Sample 3 & & 9.44 & 9.37 & 9.47 \\
\hline Sample 4 & & 9.45 & 9.43 & 9.41 \\
\hline Sample 5 & & 9.43 & 9.44 & 9.37 \\
\hline Sample 6 & & 9.44 & 9.34 & 9.47 \\
\hline Average & & 9.46 & 9.42 & 9.42 \\
\hline$s$ & & 0.04 & 0.05 & 0.04 \\
\hline
\end{tabular}


In Table 7, each value represents an average of a duplicate. The average of six samples for each combination time/temperature shows that all $\Delta \mathrm{T}_{\mathrm{m}}$ values are within the interval of the expected value and standard deviation mentioned in the kit manual $\left((10 \pm 1.5){ }^{\circ} \mathrm{C}\right.$, Roche Diagnostics, Vilvoorde, Belgium).

Therefore, all samples could be categorised as having the heterozygous genotype. In conclusion, the batch IRMM/IFCC-492 is stable at $-20{ }^{\circ} \mathrm{C}$ and contains the heterozygous G20210 wildtype/G20210A mutant sequence of a 609 bp fragment of the human prothrombin gene, that can still be identified in samples which were stored at higher temperature such as $18^{\circ} \mathrm{C}$ and $40^{\circ} \mathrm{C}$ for up to 8 weeks.

\subsection{Long-term stability}

At temperatures of $18{ }^{\circ} \mathrm{C}$ and $40{ }^{\circ} \mathrm{C}$, evaporation occurred already after 2 months, therefore the long-term stability was not investigated at these temperatures.

The long-term stability study was performed at $-20^{\circ} \mathrm{C}$ and at $4{ }^{\circ} \mathrm{C}$ for time periods of 2, 4 and 6 months (Tables 8.1 and 8.2). Six different vials were tested in triplicate (5 $\mu \mathrm{L}$ pDNA per PCR reaction) at each temperature by real-time PCR and the triplicate average is displayed in Tables 8.1 and 8.2. As two different instrument (ABI Prism $7900 \mathrm{HT}$ and $\mathrm{ABI}$ Prism 7700) were used to analyse the samples, an internal control was used on each plate to be able to compare the data, and therefore the average $\mathrm{Ct}$ value for each sample was normalised by the average value of the triplicate measurements of the control on each plate, giving $\mathrm{Ct}^{*}$ values. The average $\mathrm{Ct}$ values obtained using the ABI Prism 7700 instrument are displayed in bold. The average $\mathrm{Ct}$ value and standard deviation of the internal control (pDNA at $0.02 \mathrm{ng} / \mu \mathrm{L}$ ) was 15.73 \pm 0.13 and $16.56 \pm 0.08$ using the $\mathrm{ABI} 7900 \mathrm{HT}$ and $\mathrm{ABI}$ Prism 7700 instruments, respectively. 
Table 8.1. Long-term stability of IRMM/IFCC-492 stored for 6 months at $-70^{\circ} \mathrm{C}$

\begin{tabular}{|c|c|c|c|c|}
\hline & & \multicolumn{2}{|c|}{ Ct values } & \multirow{2}{*}{$\begin{array}{c}\Delta \mathrm{T}_{\mathrm{m}} \text { values } \\
{ }^{\circ} \mathrm{C}\end{array}$} \\
\hline & $\begin{array}{l}\text { Temperature } \\
{ }^{\circ} \mathrm{C}\end{array}$ & Ct & $\mathbf{C t}^{*}$ & \\
\hline Sample 1 & -70 & 15.80 & 1.00 & 9.31 \\
\hline Sample 2 & & 15.72 & 1.00 & 9.34 \\
\hline Sample 3 & & 15.75 & 1.00 & 9.46 \\
\hline Sample 4 & & 15.70 & 1.00 & 9.21 \\
\hline Sample 5 & & 15.74 & 1.00 & 9.19 \\
\hline Sample 6 & & 15.55 & 0.99 & 9.32 \\
\hline Average & & 15.71 & 1.00 & 9.30 \\
\hline$s$ & & 0.09 & 0.01 & 0.10 \\
\hline \multicolumn{2}{|c|}{ Control ABI 7900HT } & 15.73 & - & - \\
\hline
\end{tabular}

The data of Table 8.1 were analysed simultaneously with those in Table 8.2 as they represented the samples stored at the reference temperature. The grand average and standard deviation of the $\mathrm{Ct}^{*}$ values and $\Delta \mathrm{T}_{\mathrm{m}}$ values of samples stored at $-70^{\circ} \mathrm{C}$ is respectively $1.00 \pm 0.01$ and $(9.30 \pm 0.10){ }^{\circ} \mathrm{C}$. The $\Delta \mathrm{T}_{\mathrm{m}}$ values of Table 8.1 constitute the values obtained at the reference temperature for samples analysed in Table 9. 
Table 8.2. Long-term stability of IRMM/IFCC-492 stored for 2, 4 and 6 months at $-20{ }^{\circ} \mathrm{C}$ and at $4{ }^{\circ} \mathrm{C}$. The average $\mathrm{Ct}$ values obtained using the $\mathrm{ABI}$ Prism 7700 instrument are displayed in bold. ** indicates a value for which the average was performed only on duplicates, due to an error in pipetting.

\begin{tabular}{|c|c|c|c|c|c|c|c|}
\hline & & \multicolumn{6}{|c|}{ Ct values at $t$ (month) } \\
\hline & \multirow{2}{*}{$\begin{array}{l}\text { Temperature } \\
{ }^{\circ} \mathrm{C}\end{array}$} & \multicolumn{2}{|c|}{$t=2$} & \multicolumn{2}{|c|}{$t=4$} & \multicolumn{2}{|c|}{$t=6$} \\
\hline & & Ct & $\mathrm{Ct}^{*}$ & Ct & $\mathbf{C t}^{*}$ & Ct & $\mathbf{C t}^{*}$ \\
\hline Sample 1 & -20 & 15.65 & 1.00 & 15.81 & 1.01 & 15.75 & 1.00 \\
\hline Sample 2 & & 15.84 & 1.01 & 15.60 & 0.99 & 15.80 & 1.00 \\
\hline Sample 3 & & 15.78 & 1.00 & $15.76^{\star \star}$ & 1.00 & 15.70 & 1.00 \\
\hline Sample 4 & & 15.69 & 1.00 & 15.70 & 1.00 & 15.65 & 1.00 \\
\hline Sample 5 & & 15.60 & 0.99 & 15.72 & 1.00 & $15.71^{\star \star}$ & 0.98 \\
\hline Sample 6 & & 15.76 & 1.00 & 15.78 & 1.00 & 15.80 & 1.00 \\
\hline Average & & 15.72 & 1.00 & 15.73 & 1.00 & 15.73 & 1.00 \\
\hline$s$ & & 0.09 & 0.01 & 0.08 & 0.00 & 0.06 & 0.01 \\
\hline \multicolumn{2}{|c|}{ Control ABI 7900HT } & 15.73 & & 15.73 & & 15.73 & \\
\hline Sample 1 & 4 & 15.58 & 0.99 & 16.45 & 0.99 & 16.26 & 0.98 \\
\hline Sample 2 & & 15.72 & 1.00 & 16.47 & 0.99 & 16.27 & 0.98 \\
\hline Sample 3 & & 15.32 & 0.97 & 16.25 & 0.98 & 16.40 & 0.99 \\
\hline Sample 4 & & 15.69 & 1.00 & 16.18 & 0.98 & 16.29 & 0.98 \\
\hline Sample 5 & & 15.75 & 1.00 & 16.35 & 0.99 & 16.44 & 0.99 \\
\hline Sample 6 & & 15.70 & 1.00 & 16.52 & 1.00 & 16.43 & 0.99 \\
\hline Average & & 15.63 & 0.99 & 16.37 & 0.99 & 16.35 & 0.99 \\
\hline$s$ & & 0.16 & 0.01 & 0.13 & 0.01 & 0.08 & 0.01 \\
\hline \multicolumn{2}{|c|}{ Control ABI 7900HT } & 15.73 & - & - & - & - & - \\
\hline \multicolumn{2}{|c|}{ Control ABI 7700} & - & - & 16.56 & - & 16.56 & - \\
\hline
\end{tabular}

Scrutiny of the data: no suspicious data were detected.

The slope of the regression line and its uncertainty were calculated and tested for significance ( $t$-test; $\left.t_{0.05}, 22\right)$ using the $\mathrm{Ct}^{*}$ values. The slope was not significantly different from zero at $-20{ }^{\circ} \mathrm{C}$ but significantly at $4{ }^{\circ} \mathrm{C}\left(|\mathrm{b}| / \mathrm{u}_{\mathrm{b}}>\mathrm{t}_{\alpha, \mathrm{df}}\right.$ ), (Figure $3 \mathrm{~A}$ and 3B). 
Figure $3 \mathrm{~A}$ : at $-20^{\circ} \mathrm{C}$

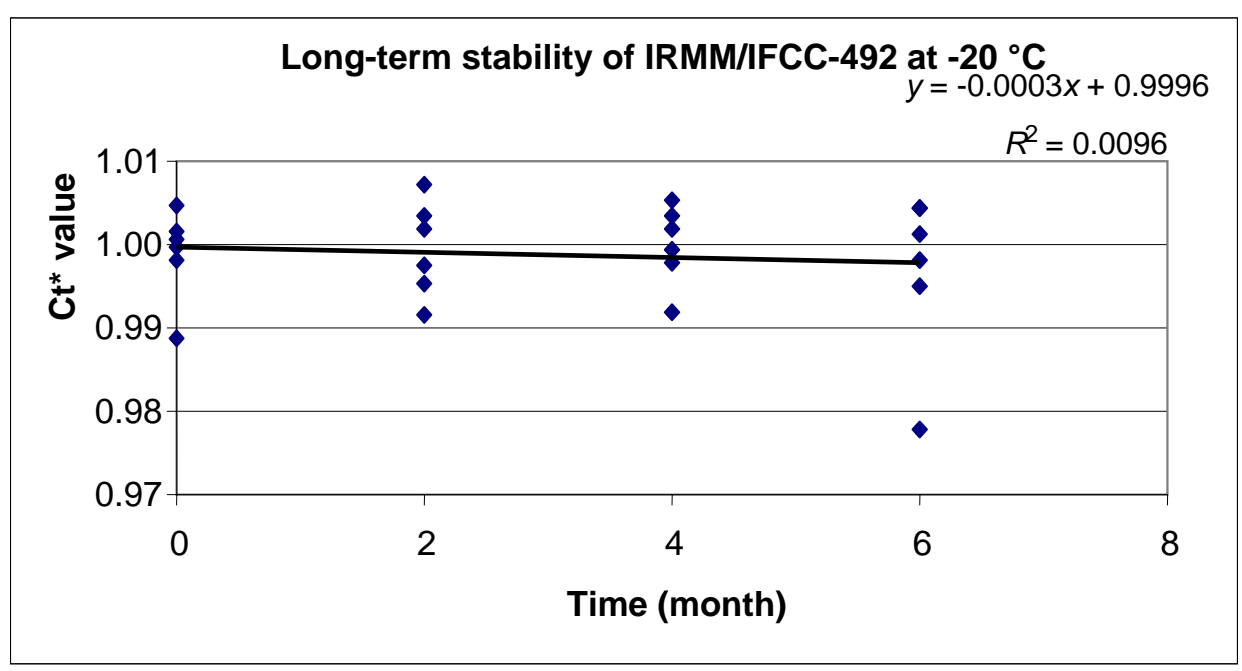

Figure 3B: at $4^{\circ} \mathrm{C}$

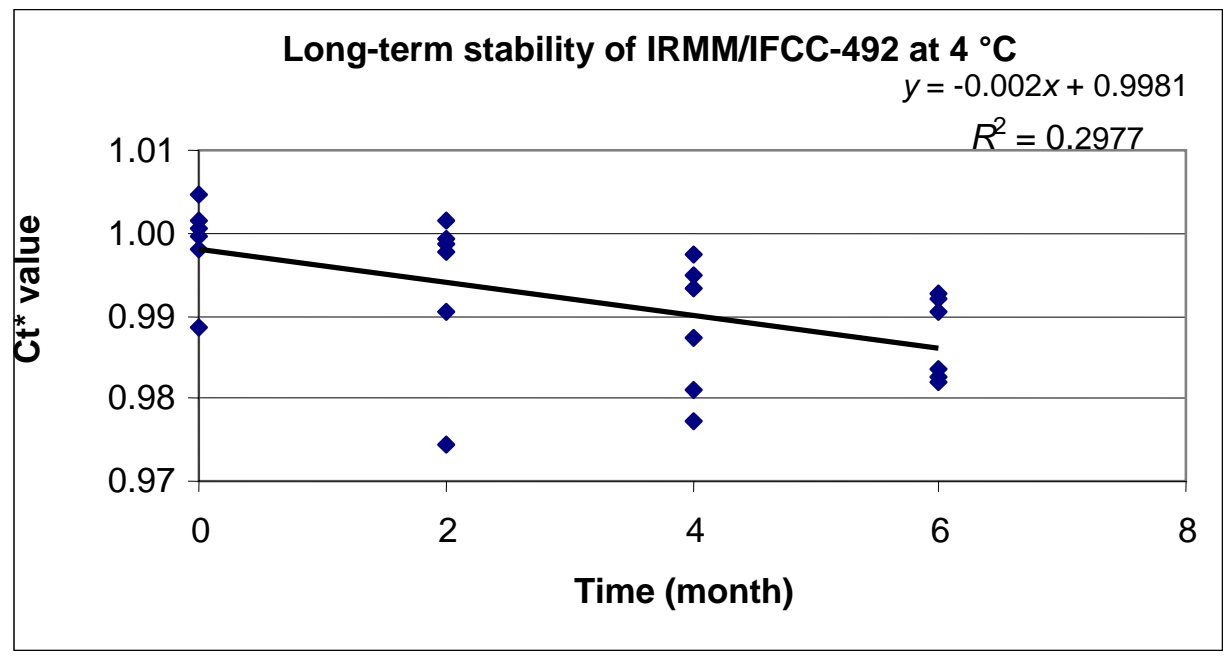

Figure 3. Slopes of the regression lines of IRMM/IFCC-492 during long-term stability study $\left(0,2,4\right.$ and 6 months at $-20^{\circ} \mathrm{C}$ and $\left.4{ }^{\circ} \mathrm{C}\right)$

Additionally, the difference in melting temperatures of the PCR products was recorded for genotyping purposes (Table 9). 
Table 9: Difference in melting temperature $I^{\circ} \mathrm{C}$ of long-term stability samples of IRMM/IFCC-492 stored for 2,4 and 6 months at $-20^{\circ} \mathrm{C}$ and at $4{ }^{\circ} \mathrm{C}$

\begin{tabular}{|l|l|l|l|l|}
\hline & & \multicolumn{3}{|c|}{$\Delta \mathbf{T}_{\mathrm{m}}$ values at $\boldsymbol{t}$ (month) } \\
\hline & $\begin{array}{l}\text { Temperature } \\
{ }^{\circ} \mathbf{C}\end{array}$ & $\boldsymbol{t}=\mathbf{2}$ & $\boldsymbol{t}=\mathbf{4}$ & $\boldsymbol{t}=\mathbf{6}$ \\
\hline Sample 1 & -20 & 9.57 & 9.41 & 9.61 \\
\hline Sample 2 & & 9.45 & 9.54 & 9.61 \\
\hline Sample 3 & & 9.52 & 9.45 & 9.61 \\
\hline Sample 4 & & 9.50 & 9.42 & 9.59 \\
\hline Sample 5 & & 9.47 & 9.41 & 9.61 \\
\hline Sample 6 & & 9.41 & 9.45 & 9.56 \\
\hline Average & & 9.49 & 9.44 & 9.59 \\
\hline S & & 0.05 & 0.05 & 0.02 \\
\hline Sample 1 & 4 & 9.41 & 9.50 & 9.49 \\
\hline Sample 2 & & 9.55 & 9.52 & 9.55 \\
\hline Sample 3 & & 9.48 & 9.60 & 9.56 \\
\hline Sample 4 & & 9.49 & 9.49 & 9.49 \\
\hline Sample 5 & & 9.44 & 9.38 & 9.55 \\
\hline Sample 6 & & 9.49 & 9.49 & 9.48 \\
\hline Average & & 9.47 & 9.49 & 9.52 \\
\hline S & & 0.05 & 0.07 & 0.04 \\
\hline
\end{tabular}

The grand average and standard deviation of the $\Delta \mathrm{T}_{\mathrm{m}}$ values of samples stored at $20{ }^{\circ} \mathrm{C}$ is $(9.51 \pm 0.08){ }^{\circ} \mathrm{C}$ and $(9.50 \pm 0.05){ }^{\circ} \mathrm{C}$ at $4{ }^{\circ} \mathrm{C}$. All $\Delta \mathrm{T}_{\mathrm{m}}$ values of the individual samples (Tables 8.1 and 9) are within the interval of the expected value mentioned in the kit manual $\left((10 \pm 1.5){ }^{\circ} \mathrm{C}\right.$, Roche Diagnostics, Vilvoorde, Belgium).

Therefore, all samples could be categorised as having the heterozygous genotype. In conclusion, the batch of IRMM/IFCC-492 is stable for 6 months at $-20{ }^{\circ} \mathrm{C}$ and contains the heterozygous G20210 wildtype/G20210A mutant sequence of a $609 \mathrm{bp}$ fragment of the human prothrombin gene. 


\section{Batch characterisation}

After plasmid construction and production, the cloned human prothrombin (Factor II) fragments inserted in the pUC18 plasmid were sequenced using DTCS protocol [9] (see [6] and the certification reports of IRMM/IFCC-490 and IRMM/IFCC-491).

\subsection{Sequencing}

The 609 bp fragment of the human prothrombin in IRMM/IFCC-490 and IRMM/IFCC491 was sequenced individually before mixing the two pDNA, using sequencing forward primers M13-F (P-0419) and Factor II-F (P-0579) and reverse primers Factor II-R (P-0580) and M13-R (P-0289), and DTCS protocol (Table 3, [9]). Forward and reverse sequence matched, therefore indicating a very low probability of misreadings $\left(p<3 \times 10^{-6}\right)$. As there was no error in the sequencing of the insert, the misreading probability was calculated as to be inferior to $1 / 609$ for the forward sequencing reaction, and inferior to $1 / 609$ when backward sequencing was performed. Therefore, the total misreadings probability in the certified sequence was calculated as inferior to $1 / 609 \times 1 / 609$.

The analysed fragments of prothrombin DNA sequence showed $100 \%$ homology with the reported DNA sequence of the prothrombin gene (GeneBank Accession number M17262) for IRMM/IFCC-490 and two point mutations (G20210A mutation at position 26784 and $A->G$ mutation at position 26628 which was confirmed to be present as well in the patient sample) for IRMM-IFCC-491, confirming the identity of produced pDNA. The sequences of IRMM/IFCC-490 and IRMM/IFCC-491 were also confirmed by sequencing using the $\mathrm{U}-20$ universal primer and an $\mathrm{ABI} 377$ instrument by Eurogentec (Liège, Belgium). 


\section{Validation}

\subsection{Field study design and results}

The performance of the material was tested in a field study by 7 diagnostic laboratories using 4 different methods routinely for the G20210A prothrombin mutation detection (Table 10). A total of eight vials labeled from $\mathrm{A}$ to $\mathrm{H}$ was sent to each laboratory. Each set of vials contained two vials of each genotype: IRMM/IFCC490 (wildtype sequence), IRMM/IFCC-491 (G20210A mutant sequence) or IRMM/IFCC-492 (heterozygous wildtype/G20210A mutant sequence generated by mixing of equal volumes of identical mass concentrations of IRMM/IFCC-490 and IRMM/IFCC-491), and two vials without DNA (Blank). The testing laboratories were provided with the study protocol, the terms to be used for reporting and the results reporting form, but had no information on the expected results or identity of the individual samples. All laboratories were asked to describe in detail the method used.

Table 10: Field study of IRMM/IFCC-490, -491 and -492 materials (Y: Yes, N: No)

\begin{tabular}{|l|c|c|l|l|l|l|}
\hline $\begin{array}{l}\text { Lab } \\
\text { code }\end{array}$ & Method & Type & \multicolumn{5}{|c|}{ Correctness of genotyping } \\
\hline & & $\begin{array}{l}\text { Homozygous } \\
\text { wildtype }\end{array}$ & $\begin{array}{l}\text { Homozygous } \\
\text { mutant }\end{array}$ & $\begin{array}{l}\text { Heterozygous } \\
\text { wildtype/G20210A } \\
\text { mutant }\end{array}$ & Blank \\
\hline $\mathbf{1}$ & PCR & LightCycler & Y & Y & Y & Y \\
\hline $\mathbf{2}$ & $\begin{array}{c}\text { Multiplex } \\
\text { PCR }\end{array}$ & Home brewed & Y & Y & Y & Y \\
\hline $\mathbf{3}$ & $\begin{array}{c}\text { Multiplex } \\
\text { PCR }\end{array}$ & Home brewed & Y & Y & Y & Y \\
\hline $\mathbf{4}$ & PCR & LightCycler & Y & Y & Y & Y \\
\hline $\mathbf{5}$ & RFLP1 & Home brewed & Y & Y & Y & Y \\
\hline $\mathbf{5}$ & PCR & LightCycler & Y & Y & Y & N \\
\hline $\mathbf{6}$ & RFLP2 & Home brewed & Y & Y & Y & Y \\
\hline $\mathbf{7}$ & PCR & Rotor-Gene & Y & Y & Y & Y \\
\hline
\end{tabular}

In Table 10, each $\mathrm{Y}$ or $\mathrm{N}$ represents agreement on the two samples that have been tested by the corresponding laboratory. All DNA-containing samples were genotyped correctly. Two vials without DNA were misclassified (one bold $\mathbf{N}$ representing the agreement on two blank samples). These data were reported as homozygous wildtype by laboratory number 5 using the LightCycler method. The same samples, tested with the in-house RFLP method by the same laboratory, were correctly identified as blank samples. The use of two different methods by laboratory number 5 was agreed before starting the study. In laboratory number 3, the samples were diluted 1:20 before use, but the dilution of the material did not influence the genotyping results. 
All vials containing pDNA (wildtype, mutant or heterozygous wildtype/G20210A mutant) were genotyped correctly and unambiguously demonstrating that the material is fit for its intended purpose.

\subsection{Methods}

Roche LightCycler: LightCycler technology from Roche, using the Factor II (prothrombin) G20210A kit and LightCycler 1.5 instrument [5].

Multiplex mutagenic separated PCR: derived from Endler et al. [11]

Thermal PCR program: $35 \times$ cycles of $95{ }^{\circ} \mathrm{C}$ for $1 \mathrm{~min}$; $54{ }^{\circ} \mathrm{C}$ for $2 \mathrm{~min} ; 72{ }^{\circ} \mathrm{C}$ for 1 min; then hold at $4{ }^{\circ} \mathrm{C}$. Primers used are described hereafter:

\begin{tabular}{|l|l|}
\hline Forward (wildtype), F2G & 5'-CAC TGG GAG CAT TGA GGC GC -3' \\
\hline Forward (mutant), F2A & 5'-ATG AAT AGT AAT GGG AGC ATT GAG GAT T -3' \\
\hline Reverse & 5'- ATG TGT TCC GCC TGA AGA AGT GGA -3' \\
\hline
\end{tabular}

PCR products $(6 \mu \mathrm{L})$ were loaded on Spreadex EL 600 gels, run at $120 \mathrm{~V}$ for $2 \mathrm{~h}$, then for $20 \mathrm{~min}$ at $40{ }^{\circ} \mathrm{C}$ to $50^{\circ} \mathrm{C}$. Staining of the gels performed with $1: 20000$ diluted SYBRGreen for $30 \mathrm{~min}$ at room temperature.

RFLP1: Derived from Poort et al. [12]

Thermal PCR program: $95^{\circ} \mathrm{C}$ for $4 \mathrm{~min}, 32 \times$ cycles of $94{ }^{\circ} \mathrm{C}$ for $1 \mathrm{~min}$; $55^{\circ} \mathrm{C}$ for 1 $\min ; 67^{\circ} \mathrm{C}$ for $1 \mathrm{~min}$; then hold at $4{ }^{\circ} \mathrm{C}$. Primers used are described hereafter:

\begin{tabular}{|l|l|}
\hline Forward & 5'-TCT AGA AAC AGT TGC CTG GC-3' \\
\hline Reverse & 5'-ATA GCA CTG GGA GCA TTG AAG C -3' \\
\hline
\end{tabular}

PCR products are digested with HindIII restriction enzyme and loaded on agarose gel for electrophoresis.

RFLP2: Derived from Danneberg et al. [13]

Thermal PCR program: $94{ }^{\circ} \mathrm{C}$ for $5 \mathrm{~min}, 35 \times$ cycles of $93^{\circ} \mathrm{C}$ for $1 \mathrm{~min}$; $58^{\circ} \mathrm{C}$ for 1 min; $72{ }^{\circ} \mathrm{C}$ for $1 \mathrm{~min} ; 72{ }^{\circ} \mathrm{C}$ for $3 \mathrm{~min}$, then hold at $4{ }^{\circ} \mathrm{C}$. Primers used are described hereafter:

\begin{tabular}{|l|l|}
\hline Forward & 5'-GCA CAG ACG GTG TTC TCT T -3' \\
\hline Reverse & 5'-ATA GCA CTG GGA GCA TTG AAG C -3' \\
\hline
\end{tabular}


PCR products are digested with HindIII restriction enzyme and loaded on agarose gel for electrophoresis.

Rotor-Gene real-time PCR analysis software 6.0: Factor II kit from Nuclear Laser Medicine, Settala, IT. 


\section{Traceability of gene fragment}

The sequence of the $609 \mathrm{bp}$ human prothrombin gene fragment inserted in the pUC18 vector to generate the IRMM/IFCC-490 and the IRMM/IFCC-491 materials was assessed by the CEQ Dye Terminator Cycle Sequencing (including a pUC18 control plasmid) and confirmed by external sequencing by Eurogentec (Liège, Belgium) using the U-20 universal primer and the ABI Prism 377 instrument. The sequence identity is traceable to PCR-based sequencing methods with very low probability of misreadings $\left(p<3 \times 10^{-6}\right)$. 


\section{Value assignment}

There is no numerical value assignment for this reference material. The sequence at position 20210 is either an A or a G, and is certified by using 2 different sequencing procedures. The uncertainty statement can be expressed as a probability that the two procedures, independently performed in different laboratories, generated identical results by forward and backward sequencing $\left(\mathrm{p}<3 \times 10^{-6}\right)$. The misreading probability was calculated as to be inferior to $1 / 609$ for the forward sequencing reaction, and inferior to 1/609 when backward sequencing was performed. Therefore, the total misreadings probability was calculated as inferior to $1 / 609 \times 1 / 609$.

Uncertainties related to homogeneity and stability studies did not jeopardize the presence of the target sequence, and it could be demonstrated that even after 8 weeks at $18{ }^{\circ} \mathrm{C}$ and at $40{ }^{\circ} \mathrm{C}$ the material could still be used in genotyping experiments without impact on the performance of the qualitative methods applied for nucleotide identification. 


\section{Instructions for use}

\subsection{Dispatch}

Dispatch to the customer should be done with cooling elements. Upon receipt by the customer, the material should be kept at $-20{ }^{\circ} \mathrm{C}$ for long-term storage or at $4{ }^{\circ} \mathrm{C}$ for use within eight weeks.

\subsection{Intended use}

This certified reference material (CRM) is intended to support the validation and the harmonisation of PCR-based methods used for detection of the G20210A mutation in the human prothrombin gene. In particular, this CRM is intended to be used as heterozygous control material in diagnostic tests for the identification and detection of the G20210A mutation in the human prothrombin gene.

Use 0.5 to $5 \mu \mathrm{L}$ of pDNA solution per 20 to $50 \mu \mathrm{L}$ total volume of the PCR reaction (depending on the method and the instrument). Alternatively, the material can be diluted 10 times prior to use, in that case $5 \mu \mathrm{L}$ of the diluted sample should be used, and no stability can be guaranteed anymore.

The plasmid DNA as provided can be kept at $4{ }^{\circ} \mathrm{C}$ for up to eight weeks or at $-20{ }^{\circ} \mathrm{C}$ for long-term storage. 


\section{References}

[1] Linsinger, T.P.J., Pauwels, J, van der Veen, A.M.H., Schimmel, H., Lamberty, A. (2001) Homogeneity and stability of reference materials. Accred Qual Assur 6, 20-25. [2] van der Veen, A.M.H., Linsinger, T.P.J., Pauwels, J. (2001) Uncertainty calculations in the certification of reference materials. 2. Homogeneity study. Accred Qual Assur 6, 26-30.

[3] van der Veen, A.M.H., Linsinger, T.P.J., Lamberty, A., Pauwels, J. (2001) Uncertainty calculations in the certification of reference materials 3. Stability study. Accred Qual Assur 6, 257-263.

[4] van der Veen, A.M.H, Linsinger, T.P.J., Schimmel, H., Lamberty, A., Pauwels, J. (2001) Uncertainty calculations in the certification of reference materials 4. Characterisation and certification. Accred Qual Assur 6, 290-294.

[5] http://www.roche-diagnostics.com/ba rmd/rmd products genomics 02.html

[6] Klein, C.L., Marki-Zay, J., Corbisier, P., Gancberg, D., Cooper, S., et al. (2005) Reference materials (RMs) for analysis of the human factor II (prothrombin) gene G20210A mutation. Clin Chem Lab Med 43(8), 862-868.

[7] Handbook of Fluorescent Probes and Research Products, Section 8.3. http://probes.invitrogen.com/handbook, Molecular Probes (2005).

[8] Corbisier, P. Validation report Working Instruction RM WI/0090, Fluorescent quantitative determination of ds DNA by PicoGreen, Internal report IRMM (2004).

[9] CEQ 8000 Series - DTCS Chemistry Protocol. http://www.beckman.com/ Literature/BioResearch/390003ac.pdf. Beckman Coulter, Inc. (2003).

[10] Endler, G., Kyrle, P.A., Eichinger, S., Exner, M., Mannhalter, C. (2001) Multiplexed mutagenically separated PCR: simultaneous single-tube detection of the factor V R506Q (G1691A), the prothrombin G20210A, and the methylenetetrahydrofolate reductase A223V (C677T) variants. Clin Chem 47, 333335.

[11] Poort, S.R., Rosendaal, F.R., Reitsma, P.H., Bertina, R.M. (1996) A common genetic variation in the 39-untranslated region of the prothrombin gene is associated with elevated plasma prothrombin levels and increase in venous thrombosis. Blood 88, 3698-3703.

[12] Danneberg, J., Abbes, A.P., Bruggeman, B.J., Engel, H., Gerrits, J., Martens, A. (1998) Reliable genotyping of the G20210A mutation of coagulation factor II (prothrombin). Clin Chem 44, 349-351. 
EUR 22167 EN - DG Joint Research Centre, Institute for Reference Materials and Measurements Certification of a Reference Material consisting of purified plasmid DNA containing a fragment from the human prothrombin gene (heterozygous G20210 wildtype/G20210A mutant)

Authors: D. Gancberg, J. Marki-Zay, P. Corbisier, C. Klein, H. Schimmel, H. Emons

Luxembourg: Office for Official Publications of the European Communities

$2006-33$ pp. $-21.0 \times 29.7 \mathrm{~cm}$

Scientific and Technical Research series

ISBN 92-79-01632-6

\begin{abstract}
This report describes the production and certification of IRMM/IFCC-492, a reference material consisting of a mix of equal volumes of identical mass concentrations of two plasmid DNA (pDNA) containing either the wildtype or the G20210A mutant sequence of a 609 base pairs fragment for the human Factor II (prothrombin) gene (IRMM/IFCC-490 and IRMM/IFCC-491). This certified reference material (CRM) is intended to support the validation and the harmonisation of polymerase chain reaction (PCR)-based methods used for detection of the G20210A mutation in the prothrombin gene. The homogeneity and short-term stability of the batch was assessed by monitoring the melting temperature $\left(T_{m}\right)$ of the product and the crossing point threshold (Cp) value obtained after PCR amplification using the LightCycler Factor II (prothrombin) G20210A kit and a LightCycler 1.5 instrument. The long-term stability was assessed using the cycle threshold value (Ct) obtained after real-time PCR amplification using specific primers and probe in combination with $\mathrm{ABI}$ Prism instruments.

The material is homogeneous and stable at $-20{ }^{\circ} \mathrm{C}$. The sequence of the insert in both plasmids (containing the wildtype sequence or the G20210A mutation) was confirmed by DNA sequence analysis. Although a second mutation (A->G) was found at position 20054 in IRMM/IFCC-491, its location did not influence the G20210A diagnostic protocol using the Factor II (prothrombin) G20210A kit. Each vial contains an indicative volume of $50 \mu \mathrm{L}$ corresponding to approximately $1 \mathrm{ng}$ of pDNA.
\end{abstract}


The mission of the Joint Research Centre is to provide customer-driven scientific and technical support for the conception, development, implementation and monitoring of European Union policies. As a service of the European Commission, the JRC functions as a reference centre of science and technology for the Community. Close to the policy-making process, it serves the common interest of the Member States, while being independent of special interests, whether private or national.

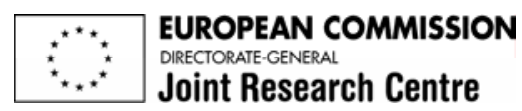


V. 


\section{Certification of reference materials for detection of the human prothrombin gene G20210A sequence variant}

\author{
David Gancberg ${ }^{1, *}$, Philippe Corbisier ${ }^{1}$, \\ Nele Meeus ${ }^{1}$, Janos Marki-Zay ${ }^{1}$, Christine \\ Mannhalter $^{2}$ and Heinz Schimmel ${ }^{1}$ \\ ${ }^{1}$ European Commission, Joint Research Centre, \\ Institute for Reference Materials and Measurements \\ (IRMM), Geel, Belgium \\ ${ }^{2}$ Medical University Vienna, Clinical Institute of \\ Medical and Chemical Laboratory Diagnostics, \\ Vienna, Austria
}

\begin{abstract}
Background: There is a need for reference materials (RMs) in the field of genetic testing for verification of test results obtained in patients and probands. For the frequent genetic variation G20210A in the prothrombin gene, it has been shown that purified plasmids containing the gene fragment harbouring the mutation constitute good candidate RMs.

Methods: Plasmid-type RMs were characterised for homogeneity, stability, sequence identity and fitness for purpose. Their certification required the use of different real-time PCR methods for genotyping and quantification of the plasmid copy number.

Results: Homogeneity, stability and fitness for the purpose of the plasmids could be demonstrated. The long-term stability (up to 24 months) of the materials was confirmed by highly sensitive and specific quantitative real-time PCR methods.

Conclusions: New types of certified RMs (CRMs) for genetic testing of the human prothrombin gene G20210A sequence variant are available. Their fitness for purpose was demonstrated and no evidence was found that they would not work with other methods as long as these are targeting the whole or parts of the prothrombin gene fragment inserted into the plasmids. The described CRMs support the efforts of the international community in development, validation and harmonisation of tests for molecular genetic testing.

Clin Chem Lab Med 2008;46:463-9.
\end{abstract}

Keywords: molecular diagnostic testing; prothrombin; real-time polymerase chain reaction (PCR); reference material; sequence variant.

\footnotetext{
*Corresponding author: David Gancberg, European Commission, Joint Research Centre, Institute for Reference Materials and Measurements (IRMM), Retieseweg 111, 2440 Geel, Belgium Phone: +32-14-571912, Fax: +32-14-571548,

E-mail: david.gancberg@ec.europa.eu Received October 10, 2007; accepted December 3, 2007; previously published online February 26, 2008
}

\section{Introduction}

Molecular genetic tests are used to identify sequence variations in patient's DNA sequence for diagnosis or prediction of inherited genetic defects. The test results may have far-reaching consequences on counselling and treatment of patients. Therefore, the use of an appropriate reference material (RM) to validate test equipment and testing methods is recommended and constitutes the basis of an adequate quality control system. Currently, the number of RMs available for genetic testing is small, presumably because such materials require by definition extensive studies to guarantee their homogeneity, stability and fitness for purpose (1). The production of a certified RM (CRM) includes the design of a RM depending on its intended use, the selection of an appropriate starting material, a feasibility study for processing and characterising the material, the preparation of the candidate RM, a homogeneity study, short- and long-term stability studies, the characterisation of the candidate RM with respect to the target properties, the assignment of the certified values and their uncertainties, and finally, the issuing of a certificate (2). While shortterm stability tests aim at determining the optimum transport conditions of the materials, longterm stability is a crucial parameter for estimating the shelf-life of a CRM under certain storage conditions. Ideally, the stability studies should follow an isochronous scheme so that all samples are measured under repeatability conditions (3). A CRM has to be accompanied by a certificate that states the value of the specified property, its associated uncertainty and a statement of metrological traceability $(1,4)$. The content of the certificate is defined in the ISO Guide $31(5)$.

The prothrombin/factor II G20210A sequence variant is a frequent genetic risk factor for venous thrombosis events (6), besides coagulation factor $\mathrm{V}$ Leiden (7).

We have previously described the use of two plasmids containing a 609-bp fragment of the human prothrombin gene (GenBank accession number M17262, nt 26302-26910) lyophilised in bovine serum albumin as candidate RMs (8). The RMs contained either the wild-type sequence (pIRMM-0001) or the G20210A mutation (pIRMM-0002) at position 26784 in the plasmid. The fitness for purpose of such plasmids as candidate RMs was demonstrated in a study involving 13 expert laboratories using nine different PCR-based methods (8). However, the stability on the long-term of the materials could not be confirmed. Now, we describe the characterisation and validation of a new 
batch of three plasmid-based RMs for the human prothrombin gene (a plasmid containing only 20210G, a plasmid containing 20210A and a mixture of the two plasmids in equal quantities). After having confirmed the identity of the DNA sequences in the plasmids (8), the homogeneity and stability on the long-term and fitness for purpose of the new batch of materials were assessed using real-time PCR on various platforms (LightCycler ${ }^{\circledR}$ or $\mathrm{ABI}$ Prism ${ }^{\circledR}$ ). Of note, an original allelic discrimination assay using real-time PCR and an ABI Prism ${ }^{\circledR}$ platform was developed in order to genotype the prothrombin fragment inserted in the plasmids. The quantitative aspects related to calibration (using prothrombin-specific assay) and stability (using vector-specific assay) of the plasmid materials were also studied.

\section{Materials and methods}

\section{Plasmid preparation}

The identity of the gene fragments inserted in the plasmids was assessed by internal and external bi-directional sequencing with a probability of misreadings estimated to be $p<(1 / 609)^{2}$. The estimate of the latter is based on the agreement between forward and reverse sequencing of the 609 -bp fragments inserted in the pUC18 vectors. The homogeneity and stability of the materials were assessed according to the requirements of the ISO Guides 34 and $35(2,9)$. The plasmids were purified using the Qiagen Plasmid Gigakit (Qiagen, Westburg, Leusden, The Netherlands) and the concentration of the stocks was assessed by the PicoGreen ${ }^{\circledR}$ double-stranded DNA quantitation method (Molecular Probes, VWR International, Leuven, Belgium). The stock solutions, with DNA concentrations of $1390 \pm 29 \mu \mathrm{g} / \mathrm{mL}$ for pIRMM-0001 and $1823 \pm 29 \mu \mathrm{g} / \mathrm{mL}$ for pIRMM-0002, were diluted to reach a final concentration of $20 \mathrm{pg} / \mu \mathrm{L}$ and aliquoted in polypropylene vials. Additionally, a mix of equal volumes of both plasmids at equal concentration was produced to mimic the heterozygous situation (the final concentration of the mix was $10 \mathrm{pg} / \mu \mathrm{L}$ for each plasmid).

\section{DNA quantitation by PicoGreen ${ }^{\circledR}$}

DNA concentrations were determined using the PicoGreen ${ }^{\circledR}$ double-stranded DNA quantitation kit (Molecular Probes), following the manufacturer's instructions. The plasmid copy number in the stock solutions was calculated on the basis of the DNA concentration and the size of the plasmids (3311 $\mathrm{bp})$, assuming that $1 \mathrm{pg}$ of double-stranded DNA corresponds to $979 \mathrm{Mbp}$.

\section{Real-time quantitative PCR (TaqMan ${ }^{\circledR}$ )}

Fluorescence labelled primers and probes for quantitative real-time PCR were designed using the Primer Express Oligo Design Software version 1.5 (Applera, Nieuwekerk aan den ljssel, The Netherlands). The primers for calibration assays (prothrombin-specific) were as follows: sense, $5^{\prime}$-CCTTTGTCATGAAGGTAAGCTTCTC-3'; antisense, 5'-TCCCCACCCCCAGAAGA-3' (300 nM final concentration); and probe, 5'-FAM-AAAGCCCAGGGCCTGGTGAACAC-TAMRA-3' (200 nM final concentration). The primers for stability assessment (vector-specific) were as follows: sense, $5^{\prime}$-GCTCACCGGCTCCAGATTT-3'; antisense, 5'-TTCTGCGCTCGGCCC-3' (300 nM final concentration); and probe, 5'-FAM-TCAGCAATAAAC-
CAGCCAGCCGGA-TAMRA-3' (200 nM final concentration). A total of $5 \mu \mathrm{L}$ of DNA sample (concentration range from ng to fg of DNA) was added to $45 \mu \mathrm{L}$ of the ready-to-use 2-times concentrated TaqMan ${ }^{\circledR}$ Universal Master Mix (Applera) containing the specific primers and probe. All samples were run in triplicate. Three blanks (no DNA added) were systematically included in each 96-well optical reaction plate. PCR was carried out in a real-time thermocycler (ABI Prism ${ }^{\circledR} 7700$ Sequence Detection System, Applera). After 1 cycle at $50^{\circ} \mathrm{C}$ for $2 \mathrm{~min}$ and $1 \mathrm{cycle}$ at $95^{\circ} \mathrm{C}$ for $10 \mathrm{~min}, 30-45$ cycles were performed each with denaturation at $95^{\circ} \mathrm{C}$ for $15 \mathrm{~s}$, annealing and elongation at $60^{\circ} \mathrm{C}$ for $1 \mathrm{~min}$. The fluorescence signal generated during PCR was recorded on-line during the amplification process and was assumed to be proportional to the number of target DNA sequences amplified. The limit of detection was calculated as $3.3 \times s \times$ lowest value in plasmid copy number in the calibration curve. The limit of quantification was calculated as $10 \times s \times$ lowest value in plasmid copy number in the calibration curve. For the 24 months long-term stability study, the samples were tested using an ABI Prism ${ }^{\circledR} 7900$ HT instrument (Applera) with the same amplification program and testing protocol.

\section{Allelic discrimination assay using minor groove binding (MGB) probes}

In the minor groove binding (MGB) assay, two probes complementary for the wild-type and the mutant allele were added simultaneously to one PCR well. Both probes consisted of an oligonucleotide containing a fluorescent reporter dye and a non-fluorescent quencher (NFQ), as well as an MGB group. TET and FAM reporter dyes (Applera) were used for the wild-type and mutated probes, respectively. The following primers and probes were used: sense, 5'-CTAAAACTATGGTTCCCAATAAAAGTGA-3'; antisense, 5'-CCATGAATAGCACTGGGAGCAT-3'; probe MGBmut, 5'-FAM-CTCAGCAAGCCTC-NFQ-MGB-3'; and probe MGBwt, 5'-TETCTCTCAGCGAGCCT-NFQ-MGB-3'. The reaction was carried out in a final volume of $25 \mu \mathrm{L}$ with $900 \mathrm{nM}$ of forward and reverse primers, $200 \mathrm{nM}$ of probes labelled with TET and FAM, $12.5 \mu \mathrm{L}$ of TaqMan ${ }^{\circledR}$ 2-times concentrated Universal Master Mix (Applera) and $5 \mu \mathrm{L}$ (range of $\mathrm{fg}$ ) of DNA template. A total of eight blanks (no DNA added) were systematically included in each 96-well optical reaction plate. PCR was carried out in a real-time thermocycler (ABI Prism ${ }^{\circledR}$ 7700 Sequence Detection System, Applera). After enzyme activation for $10 \mathrm{~min}$ at $95^{\circ} \mathrm{C}, 35$ cycles were performed each with denaturation at $95^{\circ} \mathrm{C}$ for $15 \mathrm{~s}$, annealing and elongation at $60^{\circ} \mathrm{C}$ for $1 \mathrm{~min}$. Allelic discrimination was determined by measuring fluorescence intensity at the endpoint. The results of the measurement were analysed using the SDS software version 1.9.1 (Applied Biosystems, Foster City, USA). The RMs were analysed in parallel in the same 96-well plate, using the same programme. During PCR, Rn (normalised signal) increases as amplicon copy number increases until the reaction approaches a plateau. In the read plate mode, the fluorescence signal is read at a single point in time after the completion of the PCR rather than continuously during the course of the PCR.

\section{Genotyping using the LightCycler ${ }^{\circledR}$}

The LightCycler ${ }^{\circledR}$ Factor II (Prothrombin) G20210A kit (Roche Diagnostics Belgium, Vilvoorde, Belgium, cat\# 03610195001) was used on the LightCycler ${ }^{\circledR}$ instrument (version 1.5, Roche Diagnostics), according to the manufacturer's instructions. Each reaction was prepared on ice, and $5 \mu \mathrm{L}$ of the plasmid solution $(20 \mathrm{pg})$ was added to a mix of $\mathrm{H}_{2} \mathrm{O}(11 \mu \mathrm{L})$, the 
LightCycler ${ }^{\circledR}$ detection mix $(2 \mu L)$ and reaction mix $(2 \mu L)$. For determination of the genotype after amplification [cycling programme $95^{\circ} \mathrm{C}$ for $30 \mathrm{~s} ; 45 \times\left(95^{\circ} \mathrm{C}, 55^{\circ} \mathrm{C}\right.$ for $10 \mathrm{~s}$, $72^{\circ} \mathrm{C}$ for $5 \mathrm{~s}$ )], a melting point analysis of the PCR product is performed as recommended by the manufacturer. According to the kit package insert, the acceptable values \pm intervals for the melting temperatures $\left(T_{m}\right)$ of the PCR products are $59 \pm 2.5^{\circ} \mathrm{C}$ for the wild-type allele and $49 \pm 2.5^{\circ} \mathrm{C}$ for the G20210A variant allele. The number of cycles required to pass a fluorescence threshold determines a crossing point (Cp) value which correlates with the amount of target DNA in the original sample. The $\mathrm{Cp}$ values were used to evaluate the homogeneity of the quantity of the material in each vial.

The 24 months stability data were assessed using a LightCycler ${ }^{\circledR}$ v. 2.0 instrument in combination with the software v. 4.05 and kits for in vitro diagnostic of G20210A factor II (prothrombin) mutation (Roche Diagnostics).

\section{Homogeneity study}

Although heterogeneity is not very likely for a DNA solution at the concentration level produced, the homogeneity of the RMs was assessed on the basis of the $C p$ values (Table 1) using the software MS Exce ${ }^{\circledR}$ Single Factor ANOVA (Microsoft Corporation, Silicon Valley Campus, CA, USA). After comparison of the between-bottle and within-bottle homogeneity of the samples, the relative standard uncertainty related to homogeneity was determined for each CRM. The materials homogeneity could be deduced from the samples stored at $-70^{\circ} \mathrm{C}(\mathrm{n}=6)$ and at $-20^{\circ} \mathrm{C}(\mathrm{n}=18)$ in the short-term stability study using the LightCycler ${ }^{\circledR}$ platform and kit for the G20210A variant detection. In addition, the average $T_{m}$ of the samples replicates was used for genotyping of the samples.

\section{Stability}

An isochronous scheme (3) was set up for the stability studies with $-70^{\circ} \mathrm{C}$ chosen as reference storage temperature. At least three time points were investigated at different temperatures using at least two replicates per unit in order to reduce the uncertainty related to the measurement results. After analysis, the data were screened for suspicious values (data exclusion cannot be based on statistical reasons only) and a regression line was calculated for evaluation of statistically and technically significant trends. The estimation of the uncertainty related to the stability of the material was performed at least for the planned shelf-life (10).

For the stability study on the short-term, storage at temperatures of $-20^{\circ} \mathrm{C}, 4^{\circ} \mathrm{C}, 18^{\circ} \mathrm{C}$ and $40^{\circ} \mathrm{C}$ was investigated during 2, 4 and 8 weeks ( $n=6$ at each time and temperature). For the stability study on the long-term, the storage temperatures were $-20^{\circ} \mathrm{C}$ and $4^{\circ} \mathrm{C}$ for 4,8 and 12 months, and $-20^{\circ} \mathrm{C}$ for 8,16 and 24 months ( $n=6$ at each time and temperature).

The stability of the plasmids with respect to storage time and temperature was assessed by monitoring the melting temperature values $\left(T_{m}\right.$, Table 2$)$ and measuring the cycle threshold $(\mathrm{Ct}$ ) values using real-time PCR (Tables 3 and 4).

\section{Field study}

The performance of the material was tested in a field study by seven diagnostic laboratories using four different methods routinely for the G20210A prothrombin polymorphism detection (Table 5). A total of eight vials labelled from A to $\mathrm{H}$ were sent to each participant laboratory. Each set of vials contained two vials of each genotype: IRMM/IFCC-490 (20210G sequence), IRMM/IFCC-491 (20210A sequence) or IRMM/IFCC-492 (heterozygous G20210A sequence generated by mixing of IRMM/IFCC-490 and IRMM/IFCC-491) and two vials without DNA (blank). The testing laboratories were provided with the study protocol, the terms to be used for reporting and the results reporting form, but had no information on the expected results or identity of the individual samples which had been randomised between the different laboratories. All laboratories were asked to describe in detail the method used.

Table 1 Homogeneity data of reference materials ( $C p$ and $T_{m}$ values).

\begin{tabular}{llll}
\hline Plasmid & Average $\mathrm{Cp} \pm s$ & Average $\mathrm{T}_{\mathrm{m}} \pm s,{ }^{\circ} \mathrm{C}$ & $\begin{array}{l}\text { Uncertainty contribution } \\
\text { to homogeneity, } \%\end{array}$ \\
\hline pIRMM-0001 (wild-type) & $14.64 \pm 0.18$ & $59.49 \pm 0.09$ & 0.68 \\
pIRMM-0002 (G20210A mutant) & $15.15 \pm 0.37$ & $49.97 \pm 0.08$ & 8.88 \\
plRMM-0001/pIRMM-0002 (1:1) & $15.16 \pm 0.32$ & $9.47 \pm 0.09^{*}$ & 4.73 \\
\hline
\end{tabular}

The Cp (crossing point) and $T_{m}$ (melting temperature) values $\pm s$ (standard deviation) were determined using the LightCycler $^{\circledR}$ platform and the LightCycler ${ }^{\circledR}$ Factor II (Prothrombin) G20210A kit (Roche Diagnostics). Each value represents the average of data obtained from six vials stored at $-70^{\circ} \mathrm{C}$, six vials stored at $-20^{\circ} \mathrm{C}$ for 2 weeks, six vials stored at $-20^{\circ} \mathrm{C}$ for 4 weeks and six vials stored at $-20^{\circ} \mathrm{C}$ for 8 weeks. ${ }^{*}$ This value corresponds to the difference between the melting temperature of the wildtype and the mutant sequences $\left(\Delta \mathrm{T}_{\mathrm{m}}\right)$.

Table 2 Stability study on the long-term (12 months): melting temperature $\left(T_{m}\right)$ values.

\begin{tabular}{llll}
\hline $\begin{array}{l}\text { Time (month)/ } \\
\text { temperature, }{ }^{\circ} \mathrm{C}\end{array}$ & $\begin{array}{l}\text { Homozygous wild-type } \\
\text { average } \mathrm{T}_{\mathrm{m}} \pm s,{ }^{\circ} \mathrm{C}\end{array}$ & $\begin{array}{l}\text { Homozygous mutant } \\
\text { average } \mathrm{T}_{\mathrm{m}} \pm s,{ }^{\circ} \mathrm{C}\end{array}$ & $\begin{array}{l}\text { Heterozygous } \\
\text { average } \Delta \mathrm{T}_{\mathrm{m}} \pm s,{ }^{\circ} \mathrm{C}\end{array}$ \\
\hline $0 /-70$ & $59.05 \pm 0.04$ & $49.52 \pm 0.06$ & $9.48 \pm 0.04^{*}$ \\
$4 /-20$ & $59.01 \pm 0.05$ & $49.50 \pm 0.08$ & $9.52 \pm 0.04^{*}$ \\
$8 /-20$ & $59.04 \pm 0.06$ & $49.48 \pm 0.04$ & $9.42 \pm 0.06^{*}$ \\
$12 /-20$ & $59.07 \pm 0.07$ & $49.01 \pm 0.06$ & $9.44 \pm 0.08^{*}$ \\
$4 / 4$ & $59.05 \pm 0.04$ & $49.42 \pm 0.16$ & $9.48 \pm 0.05^{*}$ \\
$8 / 4$ & $59.25 \pm 0.10$ & $49.47 \pm 0.05$ & $9.48 \pm 0.02^{*}$ \\
$12 / 4$ & $59.12 \pm 0.16$ & $49.43 \pm 0.13$ & $9.51 \pm 0.04^{*}$ \\
Combined average & $59.09 \pm 0.08$ & $49.48 \pm 0.04$ & $9.48 \pm 0.04^{*}$ \\
\hline
\end{tabular}

Each value represents the melting temperature average $\left(T_{m}\right) \pm s$ of six individual samples tested in duplicate using the LightCycler 1.5 platform and the LightCycler ${ }^{\circledR}$ Factor II (Prothrombin) G20210A kit. *These values correspond to the difference between the melting temperature of the wild-type and the mutant sequences $\left(\Delta T_{m}\right)$. 
Table 3 Stability study on the long-term (12 months): cycle threshold (Ct) values.

\begin{tabular}{llll}
\hline $\begin{array}{l}\text { Time (month)/ } \\
\text { temperature, }{ }^{\circ} \mathrm{C}\end{array}$ & $\begin{array}{l}\text { Homozygous wild-type } \\
\text { average } \mathrm{Ct} \pm s\end{array}$ & $\begin{array}{l}\text { Homozygous mutant } \\
\text { average } \mathrm{Ct} \pm s\end{array}$ & $\begin{array}{l}\text { Heterozygous } \\
\text { average } \mathrm{Ct} \pm s\end{array}$ \\
\hline $0 /-70$ & $16.08 \pm 0.07$ & $16.05 \pm 0.11$ & $16.34 \pm 0.12$ \\
$4 /-20$ & $16.07 \pm 0.08$ & $16.24 \pm 0.42$ & $16.44 \pm 0.13$ \\
$8 /-20$ & $16.12 \pm 0.07$ & $15.99 \pm 0.09$ & $16.25 \pm 0.20$ \\
$12 /-20$ & $16.09 \pm 0.09$ & $15.82 \pm 0.20$ & $16.36 \pm 0.12$ \\
$4 / 4$ & $15.89 \pm 0.07$ & $16.18 \pm 0.19$ & $16.25 \pm 0.26$ \\
$8 / 4$ & $15.21 \pm 0.15$ & $15.91 \pm 0.17$ & $15.79 \pm 0.10$ \\
$12 / 4$ & $14.90 \pm 0.32$ & $15.67 \pm 0.14$ & $15.57 \pm 0.14$ \\
\hline
\end{tabular}

Each value represents the cycle threshold average $(C t) \pm s$ of six individual samples tested in triplicate using the $A B I$ Prism $7700^{\circledR}$ and specific primers and probes.

Table 4 Stability study on the long-term (24 months): cycle threshold (Ct) values.

\begin{tabular}{llll}
\hline $\begin{array}{l}\text { Time (month)/ } \\
\text { temperature, }{ }^{\circ} \mathrm{C}\end{array}$ & $\begin{array}{l}\text { Homozygous wild-type } \\
\text { average } \mathrm{Ct} \pm s\end{array}$ & $\begin{array}{l}\text { Homozygous mutant } \\
\text { average } \mathrm{Ct} \pm s\end{array}$ & $\begin{array}{l}\text { Heterozygous } \\
\text { average } \mathrm{Ct} \pm s\end{array}$ \\
\hline $0 /-70$ & $16.26 \pm 0.14$ & $16.39 \pm 0.07$ & $16.39 \pm 0.05$ \\
$8 /-20$ & $16.25 \pm 0.09$ & $16.35 \pm 0.13$ & $16.36 \pm 0.07$ \\
$16 /-20$ & $16.28 \pm 0.16$ & $16.30 \pm 0.13$ & $16.32 \pm 0.24$ \\
$24 /-20$ & $16.09 \pm 0.12$ & $16.16 \pm 0.17$ & $16.33 \pm 0.58$ \\
\hline
\end{tabular}

Each value represents the cycle threshold average $(\mathrm{Ct}) \pm s$ of six individual samples tested in triplicate using the $A B I$ Prism ${ }^{\circledR}$ $7900 \mathrm{HT}$ and specific primers and probes.

Table 5 Field study of IRMM/IFCC-490, -491 and -492 materials.

\begin{tabular}{|c|c|c|c|c|c|c|}
\hline \multirow[t]{2}{*}{ Lab code } & \multirow[t]{2}{*}{ Method } & \multirow[t]{2}{*}{ Type } & \multicolumn{4}{|c|}{ Correctness of genotyping } \\
\hline & & & $\begin{array}{l}\text { Homozygous } \\
\text { wild-type }\end{array}$ & $\begin{array}{l}\text { Homozygous } \\
\text { mutant }\end{array}$ & $\begin{array}{l}\text { Heterozygous } \\
\text { wild-type/G20210A mutant }\end{array}$ & Blank \\
\hline 1 & PCR & LightCycler & $\mathrm{Y}$ & $\mathrm{Y}$ & $\mathrm{Y}$ & $\mathrm{Y}$ \\
\hline 2 & Multiplex PCR & Home brewed & $\mathrm{Y}$ & $\mathrm{Y}$ & $\mathrm{Y}$ & $\mathrm{Y}$ \\
\hline 3 & Multiplex PCR & Home brewed & $\mathrm{Y}$ & $\mathrm{Y}$ & $\mathrm{Y}$ & $\mathrm{Y}$ \\
\hline 4 & PCR & LightCycler & $\mathrm{Y}$ & $\mathrm{Y}$ & $\mathrm{Y}$ & $\mathrm{Y}$ \\
\hline 5 & RFLP & Home brewed & $\mathrm{Y}$ & $Y$ & $\mathrm{Y}$ & $\mathrm{Y}$ \\
\hline 5 & PCR & LightCycler & $\mathrm{Y}$ & $\mathrm{Y}$ & $\mathrm{Y}$ & $\mathrm{N}$ \\
\hline 6 & RFLP & Home brewed & $\mathrm{Y}$ & $\mathrm{Y}$ & $\mathrm{Y}$ & $\mathrm{Y}$ \\
\hline 7 & PCR & Rotor-gene & $\mathrm{Y}$ & $\mathrm{Y}$ & Y & Y \\
\hline
\end{tabular}

Two vials of each genotype and two vials without DNA (blank) were to each laboratory where a routine method was applied (of which principle and type are specified) for analysis. Each $\mathrm{Y}$ (yes) or $\mathrm{N}$ (no) symbol represents the agreement found on the two samples that have been tested by the corresponding laboratory. In laboratory number 3 , the samples were diluted 1:20 before use.

\section{Results}

Procedures for construction, sequencing and assessment of fitness for purpose of a first batch of plasmids pIRMM-0001 and pIRMM-0002 have been published previously (8). At that time the stability of these materials could not be confirmed. Now, a new approach was followed by producing plasmids in solution, stored frozen in polypropylene vials, as three CRMs (20210G, 20210A, 20210GA). The data on the findings related to homogeneity using the LightCycler ${ }^{\circledR}$ platform are summarised in Table 1. The combined average of the $T_{m}$ values obtained for each plasmid resulted in categorising pIRMM-0001 as homozygous $20210 \mathrm{G}$ variant, pIRMM-0002 as homozygous 20210A variant and their mixture as heterozygous 20210GA, according to the LightCycler ${ }^{\circledR}$ kit instructions. A plot of the $\mathrm{Cp}$ values of the PCR products vs. sample number did not reveal a trend in the filling sequence of the vials (data not shown). The slope of the regression line was not significantly different from zero (t-test at 95\% confidence interval) and the data followed a normal and unimodal distribution (data not shown), which indicated that the CRMs were homogeneous. The homogeneity-related uncertainty on the DNA quantity per vial throughout the batches was less than $10 \%$ for all three materials.

The stability of the plasmids after 12 and 24 months storage at $-20^{\circ} \mathrm{C}$ and after 12 months at $4^{\circ} \mathrm{C}$ was assessed using the LightCycler ${ }^{\circledR}$ Factor II (Prothrombin) G20210A kit for genotyping purpose and a quantitative real-time PCR method developed in-house. Using the LightCycler ${ }^{\circledR}$ platform (Table 2), the combined average of the melting temperature \pm standard deviation $\left(\mathrm{T}_{\mathrm{m}} \pm s\right)$ of our RMs obtained experimentally for six vials tested in duplicate stored at the different temperatures was $59.09 \pm 0.08^{\circ} \mathrm{C}$ for the wild-type material and $49.48 \pm 0.04^{\circ} \mathrm{C}$ for the mutant material. Concerning the mixture of both plasmids mimicking a heterozygous situation, the combined average of 
the difference in melting temperature between the wild-type and the mutant alleles $\Delta \mathrm{T}_{\mathrm{m}} \pm s$ was $9.48 \pm 0.04^{\circ} \mathrm{C}$. All plasmids preparations could be genotyped correctly after 12 months storage at $-20^{\circ} \mathrm{C}$ and $4^{\circ} \mathrm{C}$. After 24 months storage at $-20^{\circ} \mathrm{C}$, the combined average of $\mathrm{T}_{\mathrm{m}} \pm s$ of our RMs obtained experimentally for six vials tested in duplicate was $60.07 \pm 0.26^{\circ} \mathrm{C}$ for the wild-type material, $49.92 \pm$ $0.11^{\circ} \mathrm{C}$ for the mutant material and $\Delta \mathrm{T}_{\mathrm{m}} \pm s$ was found to be $10.28 \pm 0.06^{\circ} \mathrm{C}$ for the mixture of both plasmids.

In addition, the stability of the RMs was investigated after 12 and 24 months storage in a quantitative manner by recording $\mathrm{Ct}$ values obtained by real-time PCR using an $A B I$ Prism ${ }^{\circledR}$ instrument (Tables 3 and 4). For the three materials, the storage at $-20^{\circ} \mathrm{C}$ generated comparable data to those obtained after storage at the reference temperature $\left(-70^{\circ} \mathrm{C}\right)$. At $4^{\circ} \mathrm{C}$ the average $\mathrm{Ct}$ values were decreasing slightly but significantly with longer storage time. However, if the plasmid concentration would drop, the $\mathrm{Ct}$ value would increase. Thus, no loss of the plasmids occurred even after long-term storage at $4^{\circ} \mathrm{C}$. For example, the regression lines obtained after 12 months storage at $-20^{\circ} \mathrm{C}$ and at $4^{\circ} \mathrm{C}$ for plasmid pIRMM-0001 are displayed in Figure 1. Stability of the three materials for 12 and 24 months at the chosen temperatures could be demonstrated.

Calibration curves for both plasmids could be established by dilution of the stock solutions and the use of a prothrombin fragment-specific assay (Figure
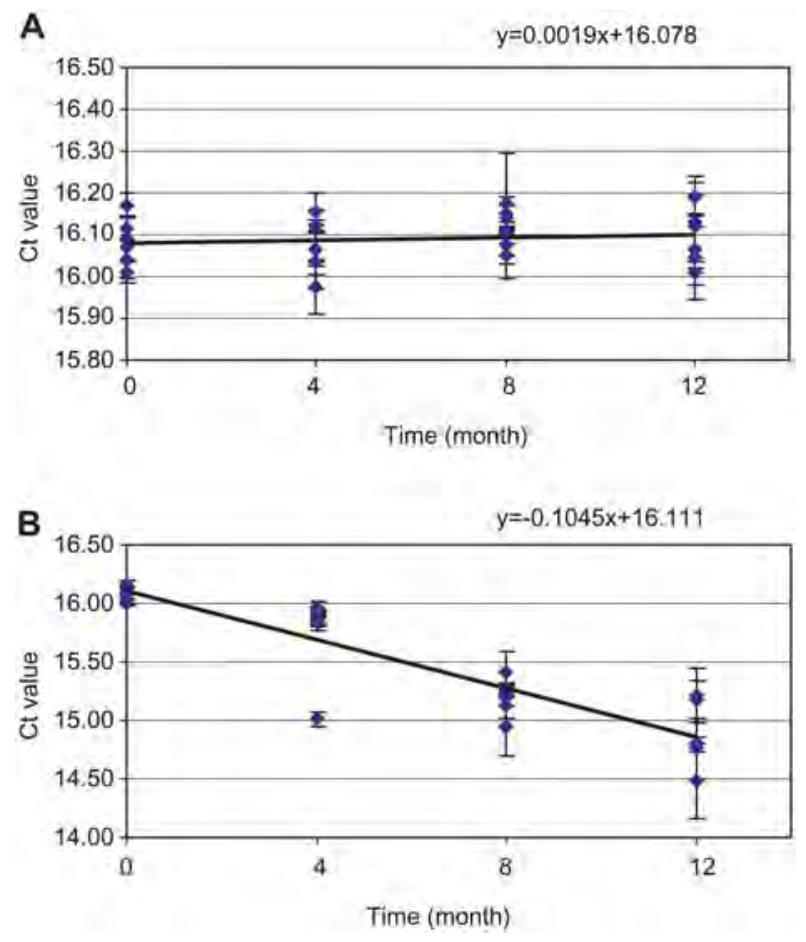

Figure 1 Stability of plasmid pIRMM-0001 stored for 12 months at $-20^{\circ} \mathrm{C}(\mathrm{A})$ or at $4^{\circ} \mathrm{C}(\mathrm{B})$.

Plasmid pIRMM-0001 stored at $-20^{\circ} \mathrm{C}(\mathrm{A})$ or at $4^{\circ} \mathrm{C}$ (B) for 12 months assessed for stability using real-time quantitative PCR. The quantitative PCR reaction was run for 30 cycles in a final volume of $25 \mu \mathrm{L}$. Each point represents the average of three measurements $\pm s$.
2). The latter showed a semi-logarithmic linear regression $\left[R^{2}=0.9884\right.$ for pIRMM-0001 (Figure 2A) and $\mathrm{R}^{2}=0.9964$ for pIRMM-0002 (Figure 2B)] over a range of plasmid copy number between 20 (corresponding to $0.07 \mathrm{fg}$ of DNA) and $10^{6}$ (corresponding to $3.4 \mathrm{pg}$ ). For example, the estimated limits of detection and quantification of the assay for the plasmid pIRMM0002 were 16 and 48 plasmid copies, respectively. The slopes for the calibration curves of the two plasmids were comparable. The allelic discrimination assay (using plasmid DNA copy number from $5 \times 10^{4}$ down to 10$)$ revealed that 50 plasmid copies could be detected and classified correctly (Figure 3 ). A clear differentiation of the three genotypes was achieved, even at low concentration, demonstrating the assay efficiency for genotyping and the suitability of the plasmids as test controls.

To test the performance of the three RMs, they were sent to diagnostic laboratories that used routinely different genotyping methods for the G20210A prothrombin polymorphism detection (Table 5). All DNAcontaining samples were genotyped correctly, unambiguously demonstrating that the materials are fit for their intended purpose. The two vials without DNA were misclassified by one laboratory using the
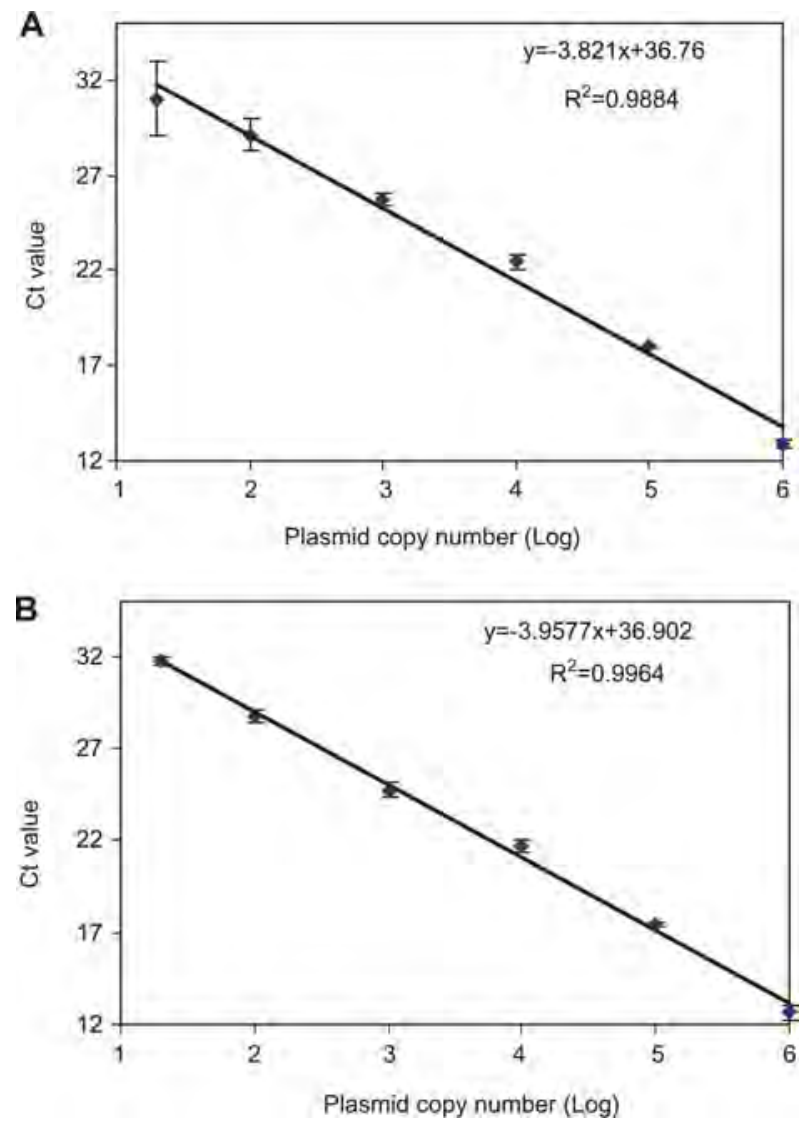

Figure 2 Calibration curve of plasmids pIRMM-0001 (A) and pIRMM-0002 (B).

Plasmids pIRMM-0001 (A) and pIRMM-0002 (B) were diluted from stock solutions and analysed in triplicate ranging from $10^{6}$ plasmid copies to 20 plasmid copies. The quantitative PCR reaction was run for 45 cycles in a final volume of $50 \mu \mathrm{L}$. Each point represents the average of three measurements $\pm s$. 


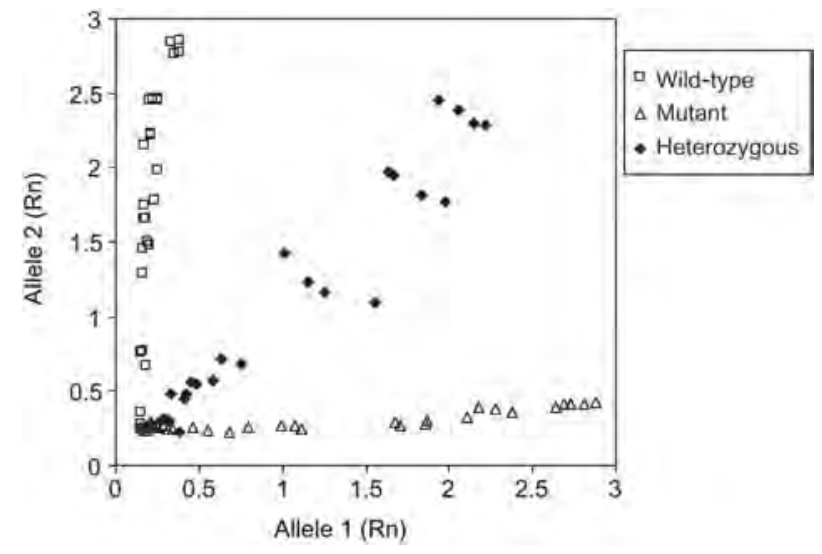

Figure 3 Allelic discrimination of plasmids pIRMM-0001 and pIRMM-0002 and their mixture in equal quantities.

Result of automatic assignment of the prothrombin genotype by real-time PCR using MGB probes and allelic discrimination programme (Applera). All plasmid dilutions $\left(5 \times 10^{4}\right.$, $5 \times 10^{3}, 5 \times 10^{2}, 50$ and 10 plasmid copies) were analysed in quadruplicates, with eight blanks and eight alleles 1 (G20210A mutant) and eight alleles 2 (20210GG wild-type) as positive controls. The axes values represent $\mathrm{Rn}$, the normalised fluorescent signal, for each allele. The fluorescence signal (proportional to the starting quantity of the material) is read at a single time point after the completion of the PCR. $\square$, homozygous wild-type; $\Delta$, homozygous mutant; $\diamond$, heterozygous wild-type/mutant.

LightCycler $^{\circledR}$ method ( $\mathrm{N}$ representing the agreement on the two blank samples). Using an in-house RFLP method, the samples were correctly identified as blank samples by this laboratory. The use of two different methods by laboratory number 5 was agreed before starting the study. The prior dilution of the material $(1: 20)$ by laboratory number 3 did not influence the genotyping results.

\section{Discussion}

Two plasmids containing a 609-bp fragment of either the wild-type 20210GG (named pIRMM-0001) or the 20210AA (named pIRMM-0002) sequence variant of the human factor II/prothrombin gene were used to generate three frozen RMs corresponding to the wildtype, mutant, and heterozygous genotypes. They were certified after assessment of their homogeneity, stability, sequence identity and fitness for purpose in a field trial.

Using a LightCycler ${ }^{\circledR}$ platform, it could be demonstrated that the materials were homogeneous and stable for up to 24 months when stored at $-20^{\circ} \mathrm{C}$ and up to 12 months at $4^{\circ} \mathrm{C}$. In other studies, the LightCycler ${ }^{\circledR}$ technology has been shown to be reliable, reproducible, sensitive and time effective for genotyping purposes, allowing the use of diluted samples (pg of genomic DNA) (11). It has been compared to standard PCR (11), the Nanochip System (12) and a real-time chemistry system using MGB-NFO probes in combination with an $\mathrm{ABI}$ Prism ${ }^{\circledR} 7700$ instrument (13). Although the performance of this method has been recognised by the scientific community, the challenge for the assessment of material stability is the use of quantitative methods, such as real-time PCR, and not only melting curve or allelic discrimination experiments.

The vector-specific real-time quantitative PCR method used for the stability assessment of the materials (Tables 3 and 4, Figure 1) showed that the concentration of the target sequence $(20 \mathrm{pg} / \mu \mathrm{L})$ did not decrease upon storage at $-20^{\circ} \mathrm{C}$ and $4^{\circ} \mathrm{C}$. On the contrary, the slight lowering of the $\mathrm{Ct}$ values during storage at $4^{\circ} \mathrm{C}$ suggested an enhancement in amplifiability or an increase in concentration of the stored materials due to evaporation of the buffer from the screw-cap polypropylene vials - possibly, structural changes occur at $4^{\circ} \mathrm{C}$. Visual inspection prior to testing did not reveal notable losses of the initial volume that would explain a decrease of $1 \mathrm{Ct}$ value after 12 months, as shown in Figure 1B. From the stability study results obtained by real-time PCR it was confirmed, for the time and temperatures investigated, that the plasmids were not degraded or lost. Of note, such assays not only allow the monitoring of measurand quantities but also the quantification of unknown samples by establishment of calibration curves. To this end, a prothrombin-specific assay was designed and the data suggested that the plasmids could be reliably used for calibration (Figure 2), provided that the plasmid copy number of the calibrant has been certified and that similar behaviour of the plasmid calibrant and the target DNA has been demonstrated. A limit of quantification between 48 and 100 plasmid copies was found for both plasmids, representing DNA quantities below the range of the femtogram.

Real-time PCR assays for allelic discrimination have been developed and proven well suited to test patients' samples (14). In the present study, we designed a prothrombin-specific TaqMan ${ }^{\circledR}$-based allelic discrimination assay using our purified plasmid RMs and were able to detect as little as $0.17 \mathrm{fg}$ plasmid DNA (corresponding to 50 plasmid copies) while retaining specificity according to the diagram set up with the homozygous alleles controls (Figure 3 ). This method combines the advantages of the LightCycler ${ }^{\circledR}$ method (genotyping) with those of the real-time PCR (i.e., quantitation).

Finally, the fitness for purpose of the CRMs was investigated and the materials performed with excellence. However, a contamination was reported by one laboratory in the blank samples (Table 5), therefore care should be taken to avoid contaminations and carry over between the CRMs and the patients' samples. To this end, storage and manipulation of the CRMs in different areas than that used for patient DNA analysis are recommended.

Even though genetic testing assays are predominantly qualitative tests, it is useful to have access to CRMs of known concentration. They can contribute to the development of primary/reference methods, ensure the traceability of measurements and support the guidelines for quality assurance in molecular genetic testing recently released (15) by international 
organisations, such as the Organisation for Economic Co-operation and Development (OECD), and the work of experts groups, such as the EuroGentest Network of Excellence (16).

In Europe, the market for in vitro medical devices (IVD) is regulated by the IVD Directive 98/79/EC, which requires that the device is fit for its intended purpose as specified by the manufacturer and traceable to higher order RMs or methods, if available. In that respect, the CRMs can be considered as the most suitable candidates to ensure this traceability requirement.

At present, besides the CRMs described above, two quality control materials are available for the detection of the $\mathrm{G} 20210 \mathrm{~A}$ variant in the prothrombin gene. The first one consists of genomic DNA that has been produced by the National Institute for Biological Standards and Control (UK) and constitutes the World Health Organization International Standard. The other one is a synthetic sequence segment of the human prothrombin gene (developed by the Maine Molecular Quality Control Inc.) recommended for monitoring of the analytical performance of the DNA extraction, amplification and detection of test systems used in qualitative measurements. Unfortunately, the characterisation data available for these two materials do not demonstrate clearly their homogeneity and stability on the long-term (there is no monitoring of the stability along sales period), but the relevant international standards for RMs, namely the ISO Guides 34 (2) and 35 (9) demand such studies. Consequently, RMs have to be assessed for their homogeneity and stability on the long-term, even if they are to be used for qualitative analysis. Indeed, the stability might become an issue if the DNA concentration drops under the detection limit of the method applied.

In conclusion, three RMs were certified for genetic testing of the G20210A point mutation in the human prothrombin gene and are now available from our Institute. They are intended to be used for validation and harmonisation of PCR-based methods used for detection of the G20210A sequence variation in the human prothrombin gene. Our results show that the strategy described in this paper seems well suited for development and production of CRMs in the field of genetic testing. Therefore, we would recommend its use for other DNA-based RMs certification.

\section{Acknowledgements}

The authors would like to thank E. Hannes (JRC-IRMM) for excellent technical assistance. We are thankful for their cooperation and useful comments to the participants of the field study: Donato Gemmati (Centre Study Haemostasis and Thrombosis, University of Ferrara, Ferrara, Italy); WalterMichael Halbmayer (Central Laboratory, Municipal Hospital Vienna-Lainz, Vienna, Austria); Steve Kitchen (Department of Coagulation, Sheffield Haemophilia and Thrombosis Centre, Royal Hallamshire Hospital, Sheffield, United Kingdom); Béla Melegh (Department of Medical Genetics and Child Development, University of Pécs, Pécs, Hungary); Michael Span- nagl (Haemostasis Division, University Hospital Munich, Germany); Renata Zadro (Clinical Institute of Laboratory Diagnosis, University School of Medicine and Clinical Hospital Centre Zagreb, Zagreb, Croatia). The project has been supported by the International Federation of Clinical Chemistry and Laboratory Medicine (IFCC) and complementary financed by the European Commission-JRC-Institute for Reference Materials and Measurements.

\section{References}

1. Emons H, Fajgelj A, van der Veen AM, Watters R. New definitions on reference materials. Accred Qual Assur 2006;10:576-8.

2. International Organization for Standardization. General requirements for the competence of reference material producers. ISO Guide 34, 2nd ed. Geneva: International Organization for Standardization, 2000 incl. Corr. 1:2003.

3. Lamberty A, Schimmel H, Pauwels J. The study of the stability of reference materials by isochronous measurements. Fresenius J Anal Chem 1998;360:359-61.

4. Emons H. The "RM family" - identification of all its members. Accred Qual Assur 2006;10:690-1.

5. International Organization for Standardization. ISO Guide 31, 2nd ed. Geneva: International Organization for Standardization, 2000.

6. De Stefano V, Chiusolo P, Paciaroni K, Casorelli I, Rossi E, Molinari E, et al. Prothrombin G20210A mutant genotype is a risk factor for cerebrovascular ischemic disease in young patients. Blood 1998;91:3562-5.

7. Rintelen C, Pabinger I, Bettelheim P, Leehner K, Kyrle PA, Knoebl P, et al. Impact of the factor II: G20210A variant on the risk of venous thrombosis in relatives from families with the factor V: R5060 mutation. Eur J Haematol 2001;67:165-9.

8. Klein CL, Marki-Zay J, Corbisier P, Gancberg D, Cooper S, Gemmati D, et al. Reference materials (RMs) for analysis of the human factor II (prothrombin) gene G20210A mutation. Clin Chem Lab Med 2005;43:862-8.

9. International Organization for Standardization. Reference materials - general and statistical principles for certification. ISO Guide 35, 3rd ed. Geneva: International Organization for Standardization, 2006.

10. Pauwels J, Lamberty A, Schimmel H. Quantification of the expected shelf-life of certified reference materials. Fresenius J Anal Chem 1998;361:395-9.

11. Schroell-Metzger B, Dicato M, Bosseler M, Berchem G. Comparison of standard PCR and the LightCycler ${ }^{\circledR}$ technique to determine the thrombophilic mutations: an efficiency and cost study. Clin Chem Lab Med 2003;41: 482-5.

12. Erali M, Schmidt B, Lyon E, Wittmer C. Evaluation of electronic microarrays for genotyping factor $\mathrm{V}$, factor II, and MTHFR. Clin Chem 2003;49:732-9.

13. Castley A, Higgins M, Ivey J, Mamotte C, Sayer DC, Christiansen FT. Clinical applications of whole-blood PCR with real-time instrumentation. Clin Chem 2005;51: 2025-30.

14. Louis M, Dekairelle AF, Gala J-L. Rapid combined genotyping of factor $\mathrm{V}$, prothrombin and methylenetetrahydrofolate reductase single nucleotide polymorphisms using minor groove binding DNA oligonucleotides (MGB probes) and real-time polymerase chain reaction. Clin Chem Lab Med 2004;42:1364-9.

15. OECD guidelines for quality assurance in molecular genetic testing. http://www.oecd.org/document/24/ 0,2340,en_2649_34537_1885208_1_1_1_1,00.html.

16. EuroGentest Network of Excellence. http://www. eurogentest.org. 
VI. 


\title{
European External Quality Control Study on the Competence of Laboratories to Recognize Rare Sequence Variants Resulting in Unusual Genotyping Results
}

\author{
János Márki-Zay, ${ }^{1,2}$ Christoph L. Klein, ${ }^{1,3}$ David Gancberg, ${ }^{1,4}$ Heinz G. Schimmel, ${ }^{1 *}$ and László Dux ${ }^{2,5}$
}

BACKGROUND: Depending on the method used, rare sequence variants adjacent to the single nucleotide polymorphism (SNP) of interest may cause unusual or erroneous genotyping results. Because such rare variants are known for many genes commonly tested in diagnostic laboratories, we organized a proficiency study to assess their influence on the accuracy of reported laboratory results.

METHODS: Four external quality control materials were processed and sent to 283 laboratories through $3 \mathrm{EQA}$ organizers for analysis of the prothrombin 20210G $>$ A mutation. Two of these quality control materials contained sequence variants introduced by site-directed mutagenesis.

RESULTS: One hundred eighty-nine laboratories participated in the study. When samples gave a usual result with the method applied, the error rate was $5.1 \%$. Detailed analysis showed that more than $70 \%$ of the failures were reported from only 9 laboratories. Allele-specific amplification-based PCR had a much higher error rate than other methods $(18.3 \%$ vs $2.9 \%$ ). The variants $20209 \mathrm{C}>\mathrm{T}$ and $[20175 \mathrm{~T}>\mathrm{G}$; 20179_20180delAC] resulted in unusual genotyping results in 67 and 85 laboratories, respectively. Eighty-three (54.6\%) of these unusual results were not recognized, $32(21.1 \%)$ were attributed to technical issues, and only 37 (24.3\%) were recognized as another sequence variant.

Conclusions: Our findings revealed that some of the participating laboratories were not able to recognize and correctly interpret unusual genotyping results caused by rare SNPs. Our study indicates that the majority of the failures could be avoided by improved training and careful selection and validation of the methods applied.

(C) 2009 American Association for Clinical Chemistry

Common functional single nucleotide polymorphisms $(\mathrm{SNPs})^{6}$ are associated with predisposition to highincidence multifactorial disorders or altered drug metabolism and play an essential role in the diagnostic process, risk stratification, and treatment selection. These genetic alterations are usually tested using DNAbased genotyping techniques, which are designed for accurate and rapid detection of the SNP of interest and not intended for mutation screening. Although these assays are highly specific, rare variants adjacent to the nucleotide of interest may cause unusual or erroneous genotyping results (1-5). Such rare variants are known, for example, in genes coding factor $\mathrm{V}(6-8)$, hemochromatosis $(9,10)$, factor II (prothrombin) $(5,11-14)$, and cholesterol ester transfer protein (CETP) (1). However, their influence on the accuracy of reported laboratory results has not yet been investigated.

The Institute for Reference Materials and Measurements (IRMM) and the Department of Biochemistry of the University of Szeged agreed on a proficiency testing (PT) study in cooperation with 3 European external quality assessment (EQA) organizations to assess how clinical laboratories interpret unusual genotyping results and how many of these laboratories recognize interfering mutations and report them correctly. Additional aims of the study were to identify weaknesses of genetic testing services, to develop QC materials for rare variants, and to gather more information about the requirements for DNA-based candidate reference materials (RMs).

\footnotetext{
${ }^{1}$ European Commission, Joint Research Centre, Institute for Reference Materials and Measurements, Geel, Belgium; ${ }^{2}$ Department of Biochemistry, Faculty of Medicine, University of Szeged, Szeged, Hungary; ${ }^{3}$ European Commission, Joint Research Centre, Institute for Health and Consumer Protection, Ispra, Italy; ${ }^{4}$ European Commission, Directorate-General for Research, Health Biotechnology, Brussels, Belgium; ${ }^{5}$ QualiCont Kht., Szeged, Hungary.

* Address correspondence to this author at: European Commission, Joint Research Centre, Institute for Reference Materials and Measurements, Retieseweg 111, B-2440 Geel, Belgium. Fax +32 14 571548; e-mail Heinz.Schimmel@
}

ec.europa.eu.

Received July 8, 2008; accepted January 27, 2009.

Previously published online at DOI: 10.1373/clinchem.2008.112102

${ }^{6}$ Nonstandard abbreviations: SNP, single nucleotide polymorphism; CETP, cholesteryl ester transfer protein; IRMM, Institute for Reference Materials and Measurements; PT, proficiency testing; EQA, external quality assessment; RM, reference material; CRM, certified reference material; LDT, laboratory-developed test; FRET, fluorescence resonance energy transfer; $A D$, allelic discrimination; dHPLC, denaturing HPLC. 
Recently, in a collaborative effort, the Scientific Committee of Molecular Biology Techniques in Clinical Chemistry of the IFCC and the IRMM have developed a set of 3 plasmid-type certified reference materials (CRMs) for the analysis of the 20210G >A (G20210A) mutation in F2, the coagulation factor II (prothrombin) gene (15-19). These plasmids contain a wild-type or G20210A mutant prothrombin gene fragment that spans all primer annealing sites published to date.

The G20210A mutation in the 3' untranslated region of the prothrombin gene is associated with higher mean plasma prothrombin concentrations and increased risk for thrombotic diseases (20). The clinical significance of the presence of this SNP, indications for testing, and therapeutic consequences of the results have been reviewed (21). Because no specific functional (e.g., clotting) tests exist, the analysis of this SNP in the course of initial evaluation of suspected inherited thrombophilia is one of the most frequently performed genetic tests in clinical laboratories; all the EQA organizations contacted offer surveys for this test in their molecular diagnostic schemes.

Other variants of the prothrombin gene, such as $20209 \mathrm{C}>\mathrm{T}(22,23)$ (C20209T), 20209C $>\mathrm{A}(24)$, $20207 \mathrm{~A}>\mathrm{C}(25), 20218 \mathrm{~A}>\mathrm{G}(14)$, and $20221 \mathrm{C}>\mathrm{T}$ (12), are relatively rare (detected in 1 in 1660 samples). The C20209T mutation accounts for nearly $85 \%$ of these variants. These and similar mutations can result in unusual genotyping results, and the correct reporting and interpretation of these cases could be an important indicator of the competence of testing laboratories.

\section{Materials and Methods}

\section{PRODUCTION OF THE EXTERNAL QC SAMPLES}

Development and processing of the wild-type and G20210A mutant plasmid CRMs has been reported (15). These plasmids include a well-characterized fragment of the human prothrombin gene (GenBank M17262; nucleotide position 26 302-26 910) comprising the SNP of interest and spanning all published primer annealing sites.

We introduced the C20209T mutation into the wild-type sequence using the QuikChange II SiteDirected Mutagenesis Kit (Stratagene) according to the manufacturer's recommendations, with 2 complementary oligonucleotides as mutagenesis primers. The sense oligo was $5^{\prime}$-CCCAATAAAAGTGACTCTCAG TGAGCCTCAATGCTCCCAGTGC-3C' (mutagenic nucleotide is underlined) (Proligo Biochemie $\mathrm{GmbH}$ ). Plasmids were transfected and cultured in XL1-Blue supercompetent cells provided in the kit.

We screened plasmid preparations purified from the cultured and harvested bacteria using the Qiagen
QIAfilter Plasmid Maxi Kit (Qiagen Benelux B.V.) for the presence of mutations resulting in unusual melting curves on the LightCycler ${ }^{\circledast}$ with the Prothrombin (G20210A) Mutation Detection Kit (Roche Diagnostics) according to the kit insert. Two plasmids containing newly introduced mutations were sequenced using suitable primers (Profw, 5'-GCACAGACGGCTGT TCTCTT-3', and Prorev, 5' -CCCGAGTGCTCGGAC TACCA-3'; synthesized by VBC Genomics, Vienna, Austria; HPLC-purified) and CEQ Dye Terminator Cycle Sequencing Kit with the Beckman CEQ8000 Sequencer (Analis) according to the manufacturer's instructions. Purity of plasmid preparations was confirmed by the OD 260/280 ratio, and DNA concentration was determined using an Eppendorf Biophotometer (VWR International). We mixed plasmids containing the newly introduced mutations with equal amounts of the G20210A mutant or wild-type plasmids. These plasmid preparations were diluted in Tris-EDTA buffer $(10 \mathrm{mmol} / \mathrm{L}, 1 \mathrm{mmol} / \mathrm{L}, \mathrm{pH} 8.0)$ to a final concentration of approximately $10 \mathrm{pg} / \mu \mathrm{L}$ DNA. Before aliquoting these diluted stocks, we tested them again on the LightCycler system as described above.

\section{DESIGN OF THE RING TRIAL}

Four external quality control materials were aliquoted (30 $\mu \mathrm{L} /$ vial) into sterile, self-standing, high-recovery $1.5-\mathrm{mL}$ polypropylene screw-cap vials and distributed to each of the 283 laboratories participating in 3 European EQA schemes (DGKL Referenzinstitut für Bioanalytik, Bonn, Germany; Instand e.V., Düsseldorf, Germany; and QualiCont Kht., Szeged, Hungary). The actual number of laboratories invited for this exercise was likely lower due to overlapping coverage of the same laboratories by multiple schemes.

Two of the 4 materials contained mutations giving unusual genotyping results when using some methods: sample A, 20210A/[20175T>G; 20179_20180delAC], heterozygous for the G20210A mutation; sample B, G20210/C20209T, homozygous wild-type for the G20210A mutation; sample C, 20210A/20210A, homozygous for the G20210A mutation; and sample D, G20210/G20210, homozygous wild-type for the G20210A mutation.

Participants were informed that the external QC samples contained plasmid-type materials but were given no information on the expected results. Using a standard form, testing laboratories were asked to give information on the type of the center activity, spectrum of the tests offered, accreditation status of the laboratory, number of genetic tests performed yearly (factor II G20210A and in general); dates of arrival and testing; storage conditions; and incidental comments/observations on the samples. The laboratories were asked to describe in detail the methods applied (including the sample processing protocol 


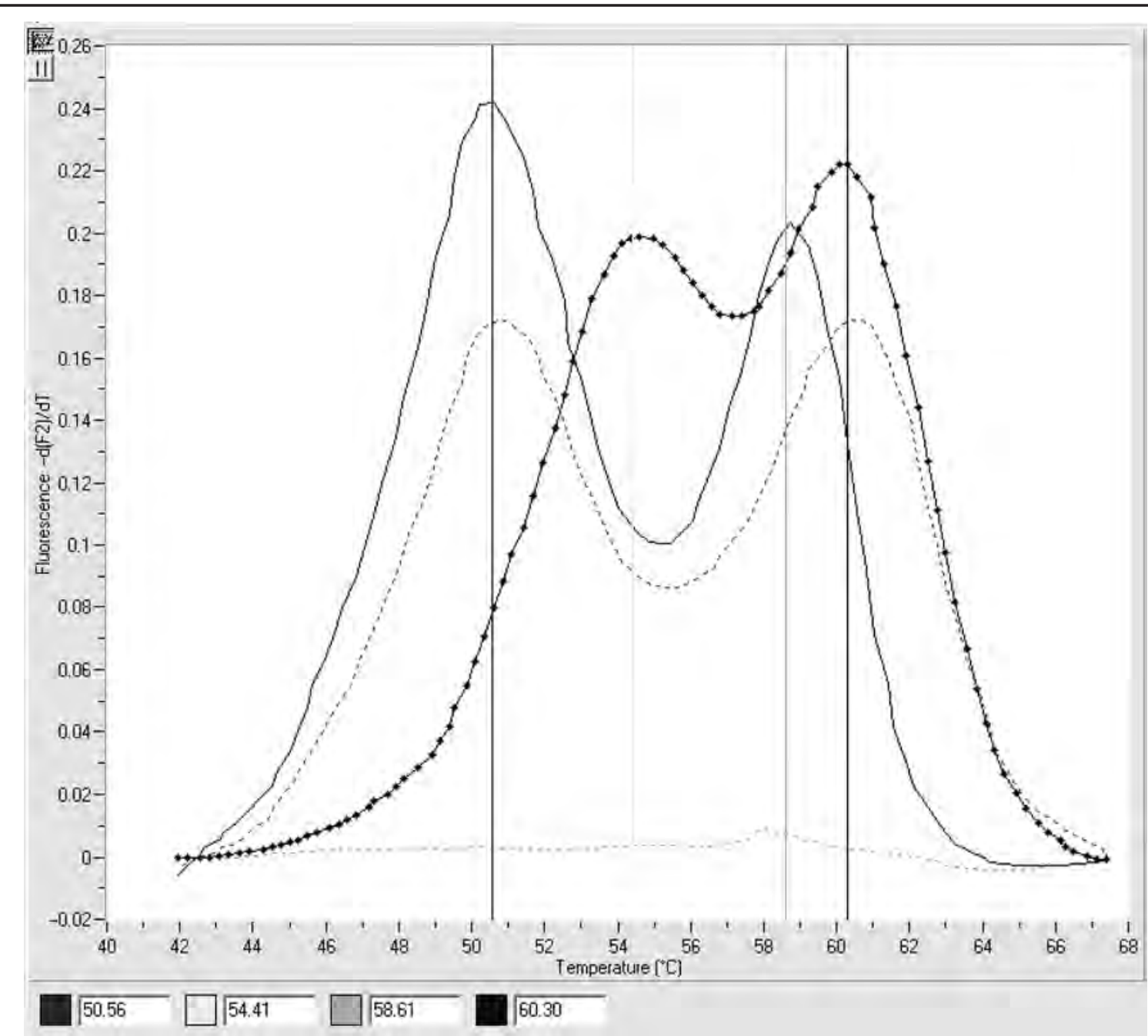

Fig. 1. Melting curves on samples A and B using LightCycler and factor II (prothrombin) G20210A mutation detection kit.

—, sample A (20210A/[T20175G;20179_20180delAC]); -O-, sample B (C20209T/wild-type); --. , no template; - - -, heterozygous control. Vertical lines indicate melting temperatures.

and genotype and origin of the controls used) and submit their genotyping results including the raw data (e.g., copy of the gels, melting curves). No standardized terms for the reporting were defined. Further comments on the results were welcomed.

Statistical analysis of the study results was performed by $\chi^{2}$-test, and the significance limit was set to $P<0.05$.

\section{Results}

PRODUCTION OF THE NEW QC MATERIALS

Screening of plasmid preparations detected 2 new variants displaying unusual melting peaks at approximately $54{ }^{\circ} \mathrm{C}$ and $58{ }^{\circ} \mathrm{C}$. Sequencing of these plasmids identified the newly introduced mutations as C20209T and [20175T > G; 20179_20180delAC] mutations, respectively. Of note, the second mutation was not introduced by site-directed mutagenesis, but occurred accidentally. This quite complex variation leads to a decrease of the melting temperature of the anchor probe, resulting in a relatively sharp melting peak. Melting curves obtained using LightCycler on the ring trial samples A (20210A/[20175G; 20179_20180delAC]) and B (G20210/20209T) are shown in Fig. 1.

\section{PROFICIENCY TESTING STUDY}

After removal of duplications resulting from the overlap of laboratories participating in more than 1 EQA 
Table 1. Details on participating laboratories.

\begin{tabular}{|lr|}
\hline & $\mathbf{n}$ \\
\hline Type of center activity (of 188 answering) & 48 \\
University & 3 \\
Research & 67 \\
Hospital & 67 \\
Independent laboratory & 3 \\
Manufacturer & \\
Spectrum of clinical tests offered (of 180 answering) & 129 \\
General & 36 \\
Only genetic tests & 15 \\
Other (e.g. Hemostasis) & \\
Conditions tested (of 115 answering) & 61 \\
Only risk factors & 54 \\
Monogenic diseases and risk factors & \\
Accreditation status (of 179 answering) & 26 \\
ISO 17025 & 43 \\
ISO 15189 & 41 \\
Other (e.g. ISO 9001) & 104 \\
Accredited & 75 \\
Not accredited & \\
\hline
\end{tabular}

scheme, 189 laboratories from 21 countries participated in the survey. Some features of the participants are shown in Table 1.
These laboratories applied a large variety of genotyping techniques, representing 50 different analytical procedures. All of the assays could amplify the target sequence from the plasmids, proving that the samples were suitable for the interlaboratory comparison exercise.

Genotyping results are summarized in Table 2 and can be split into usual and unusual results after analysis. Usual results have typical genotyping outcomes (e.g., in terms of sequence) or test system responses (e.g., melting curve or bands on the electropherogram), which correspond to one of the expected genotypes, namely homozygous wild-type (G20210/G20210), heterozygous (G20210/20210A), or homozygous G20210A mutant (20210A/20210A) with the surrounding sequence being conserved and being present in the vast majority of individuals. Results are called unusual when the presence of a sequence variant or the test system response did not correspond to expectations for the majority of individuals, such as abnormal melting curves, unexpected nucleotide sequences, or a band of unanticipated size in the electropherogram.

More detailed analysis of the results showed that the performance of the individual laboratories did not depend on the type of the laboratory, the spectrum of tests offered, or the accreditation status of the laboratory (data not shown). Table 3 displays the use of different genotype controls in the participant laboratories.

Table 2. Overview of genotyping results and error rates.

\begin{tabular}{|c|c|c|c|c|c|}
\hline & Sample $A^{a}$ & Sample B & Sample C & Sample D & Total \\
\hline Usual results, $\mathrm{n}^{\mathrm{b}}$ & 112 & 104 & 189 & 189 & 596 \\
\hline Correct, $\mathrm{n}$ & 102 & 100 & 177 & 180 & 559 \\
\hline False, $n$ & 7 & 3 & 12 & 8 & 30 \\
\hline Not reported, n & 3 & 1 & 0 & 1 & 5 \\
\hline Error rate, \% & 6.4 & 2.9 & 6.3 & 4.3 & 5.1 \\
\hline Unusual results, $\mathrm{n}^{\mathrm{c}}$ & 67 & 85 & $N A^{d}$ & NA & 152 \\
\hline Not described (false), $\mathrm{n}(\%)^{\mathrm{e}}$ & $50(74.6)$ & $33(38.8)$ & NA & NA & $83(54.6)$ \\
\hline Technical issue, $\mathrm{n}(\%)^{\mathrm{f}}$ & $10(14.9)$ & $22(25.9)$ & NA & NA & $32(21.1)$ \\
\hline Recognized variant, $\mathrm{n}(\%)$ & $7(10.5)$ & $30(35.3)$ & NA & NA & $37(24.3)$ \\
\hline \multicolumn{6}{|c|}{$\begin{array}{l}\text { a Without allelic discrimination. } \\
\text { b Results where the test system response (e.g., melting curve or electropherogram) were as expected and corresponding to homozygous wild-type (G20210/G20210), } \\
\text { heterozygous (G20210/20210A), or homozygous G20210A mutant (20210A/20210A) genotype. These results were not influenced by the sequence variants in } \\
\text { samples A and B. } \\
\text { c In } 67 \text { and } 85 \text { laboratories for samples A and B, respectively, the sequence variants affected the methods, and unusual genotyping results (e.g. abnormal melting } \\
\text { curves or electropherogram, unexpected nucleotide changes in sequencing data) were obtained that should have been detected and reported accordingly. Some } \\
\text { of these unusual results were detected and reported either as "technical issues" or "recognized variants"; however, many participants did not describe these } \\
\text { atypical observations and reported the results as one of the expected genotypes without additional remarks. } \\
\text { d NA, not applicable. } \\
\text { e Reported as one of the expected genotypes, but the sequence variant resulted in an atypical genotyping pattern using the technique applied. } \\
\text { f Genotypes not reported but observations described such as unusual results of presumed technical origin. }\end{array}$} \\
\hline
\end{tabular}




\begin{tabular}{|lc|}
\hline \multicolumn{2}{|c|}{$\begin{array}{c}\text { Table 3. Genotype controls used in the } \\
\text { participating laboratories. }\end{array}$} \\
\hline \multicolumn{2}{|c|}{ Positive control } \\
Kit control & 106 \\
G20210/20210A patient DNA & 35 \\
$\begin{array}{l}\text { G20210/G20210 and G20210/20210A } \\
\text { patient DNA }\end{array}$ & 18 \\
$\begin{array}{l}\text { G20210/G20210 and 20210A/20210A } \\
\text { patient DNA }\end{array}$ & 1 \\
\hline $\begin{array}{l}\text { G20210/G20210, G20210/20210A, } \\
\text { and 20210A/20210A patient DNA }\end{array}$ & 14 \\
\hline $\begin{array}{l}\text { G20210/20210A patient DNA and 5 } \\
\text { plasmids for rare SNPs }\end{array}$ & 1 \\
\hline $\begin{array}{l}\text { Ring trial samples } \\
\text { NIBSC-WHO reference panel }\end{array}$ & 3 \\
\hline $\begin{array}{l}\text { No data } \\
\text { a NIBSC, National Institute for Biological Standards and Control. }\end{array}$ \\
\hline
\end{tabular}

USUAL RESULTS

Genotyping assays for detection of the G20210A mutation in the prothrombin gene usually result in the genotypes homozygous wild-type (G20210/G20210), heterozygous (G20210/20210A), or homozygous G20210A mutant (20210A/20210A). All the methods provided such results on samples $C$ and $D$, and the majority of the techniques also on samples $\mathrm{A}$ and $\mathrm{B}$. Among these results, the error rate was $5.1 \%$, which corresponds to previous observations in the literature (26-29). Interestingly, the error rates were higher on samples $\mathrm{A}$ and $\mathrm{C}$ containing the G20210A mutation (6.4\% and 6.3\%, respectively) than on samples B and D without the G20210A mutation $(2.9 \%$ and $4.3 \%$, respectively); however, this difference was not statistically significant.

Currently, commercial kits as well as several laboratory developed tests (LDTs) are used for identification of the prothrombin G20210A mutation. Table 4 presents the error rates on usual results using the different techniques. The most frequently applied methods were LightCycler [fluorescence resonance energy transfer (FRET)], reverse hybridization, and PCR-RFLP. Reverse hybridization assays were carried out using commercial kits. Sixty of the 78 LightCycler users applied the Roche Factor II (Prothrombin) G20210A mutation detection kit. Among PCR-RFLP methods, most of the laboratories applied the original method published by Poort et al. (20) or its slightly modified versions, whereas only 4 laboratories used an improved method (30), which contains an additional restriction site for digestion control. Although more laboratories used commercial kits for PCR-RFLP and allele-specific PCR than LDTs, approximately $63 \%$ of the prothrombin G20210A tests were performed in laboratories employing LDTs. It should be
Table 4. Methods used and error rates.

\begin{tabular}{|c|c|c|}
\hline Method groups & $\mathrm{n}$ & Error rate \\
\hline \multicolumn{3}{|l|}{$\begin{array}{c}\text { Commercial/laboratory } \\
\text { developed tests }\end{array}$} \\
\hline Commercial & 111 & $16 / 320(5.0 \%)$ \\
\hline LDT & 78 & $14 / 270(5.2 \%)$ \\
\hline \multicolumn{3}{|l|}{ Method principle } \\
\hline PCR-RFLP & 34 & $2 / 134(1.5 \%)^{a}$ \\
\hline LightCycler & 78 & $7 / 155(4.5 \%)$ \\
\hline Allele-specific PCR & 15 & $11 / 60(18.3 \%)$ \\
\hline Reverse Hybridization & 43 & $5 / 169(3.0 \%)$ \\
\hline Allelic Discrimination & 10 & $1 / 30(3.3 \%)^{b}$ \\
\hline Sequencing & 7 & $0 / 19$ \\
\hline Denaturing HPLC & 1 & $0 / 2$ \\
\hline Molecular beacon & 1 & $0 / 4$ \\
\hline \multicolumn{3}{|c|}{$\begin{array}{l}{ }^{a} \text { Without the } 4 \text { false results reported incorrectly due to inadequat } \\
\text { nomenclature. } \\
\text { b Only the results on the Sample B. }\end{array}$} \\
\hline
\end{tabular}

emphasized that the difference in error rates for commercial and laboratory-developed tests was not found to be statistically significant.

Allele-specific amplification PCR showed a much higher error rate than other methods ( $18.3 \%$ vs $2.9 \%)$, and 6 of 15 laboratories (40\%) using this technique reported false results. No statistically significant differences in performance were seen between commercial kits and LDTs. Although these findings cannot be explained only by shortcomings of the technique, the method appears to be less reliable.

Altogether, 30 incorrect genotypes were reported by 19 participants for results with a usual genotyping pattern (Table 4). Further analysis of these results showed that $21(70 \%)$ of these failures were obtained from only 9 laboratories.

Two of these laboratories reported the results using inappropriate nomenclature indicating only the presence or absence of the G20210A mutation and did not distinguish the heterozygous from the homozygous G20210A mutation. Such results were not considered to be adequately and correctly reported (31) because the different risk levels associated with the heterozygous or homozygous genotypes may influence clinical decisions.

Careful comparison of available raw data and genotypes reported revealed that 2 participants mixed up findings postanalytically. In 2 other cases, the genotypes were assigned incorrectly, but the raw data showed technically correct results. One laboratory employing an allele-specific PCR kit submitted results despite having incorrect findings on the control samples. 
One participant using an inappropriately designed LightCycler assay mistyped 3 samples.

\section{UNUSUAL RESULTS}

Detailed analysis of the results obtained for sample A revealed that the [20175T>G; 20179_20180 del AC] mutation interfered with the assay applied in 67 laboratories, leading to unusual genotyping results. In addition, most results from the 10 laboratories using the TaqMan allelic discrimination (AD) assays indicated an impaired amplification of the wild-type sequence due to the [20175T>G; 20179_20180delAC] mutation.

Sixty laboratories employed the LightCycler instrument with the LightCycler Prothrombin G20210A Mutation Detection Kit (Roche), and 1 more participant used a laboratory-developed method similar to that applied in this kit. Using these methods, the [20175T>G; 20179_20180delAC] mutation led to a decrease of the melting temperature of the anchor probe, which resulted in a melting peak at approximately $1.7^{\circ} \mathrm{C}$ lower than expected for the wild-type allele (Fig. 1). Although this peak was inside the range of $59(2.5)^{\circ} \mathrm{C}$ indicated by the manufacturer, the difference between the wild-type and the mutant melting peaks did not fall into the range specified in the kit instructions $\left[10(1.5)^{\circ} \mathrm{C}\right]$. Probably because of the small deviation from the expected values, only $23 \%$ of the laboratories reported unusual melting peaks on this sample. Approximately half of these laboratories noted that such unusual melting peaks could indicate a variant in the probe region.

Three laboratories used sequencing as their routine procedure. In addition, one of the LightCycler users, observing the unusual melting curves, sequenced the region to identify the variant. All 4 laboratories could identify the mutation, although in 1 case it was not correctly described.

One laboratory applied a denaturing HPLC (dHPLC) assay. This participant detected the presence of the variant, but mistyped the 20210A mutant allele as wild-type in sample A.

Two laboratories using PCR-RFLP methods with high-resolution electrophoresis (PAGE or Agilent Labon-chip) found a double band instead of the expected single band on the wild-type allele. This second band probably corresponded to the undigested heteroduplex of the 2 alleles, which had a moiety with lower electrophoretic mobility due to the loop arising from the mismatches. Both laboratories noted this unusual finding.

Ten of the participating laboratories used different TaqMan AD assays. Seven of these laboratories reported a homozygous mutant (20210A) genotype on sample A, 1 participant genotyped the sample as heterozygous (G20210/20210A), and 2 laboratories, upon finding unusual $\mathrm{AD}$ plots, genotyped the sample by PCR-RFLP. The few available raw data (for 2 of the 10 laboratories) revealed that the sequence alteration in the sample A led to a shift in the AD plot depending on the primer sequences. In cases where $\mathrm{AD}$ plots were affected, amplification of the wild-type sequence was probably inefficient because of the impaired primer binding due to the [20175T>G; 20179_20180delAC] mutation. The lack of raw data did not allow further evaluation of the results from $\mathrm{AD}$ assays on sample $\mathrm{A}$.

The C20209T mutation in sample B gave unusual genotyping results in 85 laboratories. Seventy-eight of these laboratories applied the LightCycler technique with 6 different methods. Owing to the C20209T mutation, the melting peak was located at approximately $54^{\circ} \mathrm{C}$ using the Roche Factor II (Prothrombin) G20210A mutation detection kit. In 3 published LightCycler-based assays, the melting peak is at approximately $60^{\circ} \mathrm{C}$ for the wild-type allele, whereas for the G20210A mutation, it is located close to $53^{\circ} \mathrm{C}$. Using these methods, the C20209T variant appeared as a peak at approximately $50.5^{\circ} \mathrm{C}$; however, this peak was smaller than the usual ones, which might render the evaluation of these results slightly more difficult.

Seven participants tested the samples by sequencing as standard procedure. Four of these assays were minisequencing techniques (e.g., pyrosequencing) determining only a few bases adjacent to the mutation of interest. One of the pyrosequencing methods was not able to detect the C20209T mutation. Only 1 of 6 other laboratories performing sequencing reported the C20209T mutation, although it could clearly be identified on the basis of the raw data provided. Two participants sequenced this sample because of the unusual melting curves they obtained using the LightCycler. The returned sequences identified the C20209T mutation.

One laboratory used PCR-dHPLC technique without and with addition of wild-type sequences. This participant reported sample B as heterozygous for the G20210A mutation (wild-type/20210A) because this sample gave a chromatographic pattern that could not be distinguished from the heterozygous G20210A samples.

Although a majority of laboratories did not report when they experienced unusual results on samples $A$ and $\mathrm{B}$, other participants recognized the presence of the sequence variants or attributed the unusual behavior of the assay to technical issues, including poor quality or low concentration of the DNA. The other techniques used for genotyping in 93 of 179 laboratories $(52.0 \%)$ were not influenced by the additional mutations in samples A and B.

\section{Discussion}

Both method validation and internal/external quality control require samples of known genotypes. However, access to samples carrying rare genotypes (such as the 
homozygous 20210A mutation in the prothrombin gene) or harboring rare SNPs (such as the $20209 \mathrm{~T}$ allele) is limited (32). The restricted availability may contribute to the relatively high number of failures in the identification of G20210A mutant genotypes (both heterozygous and homozygous forms). Therefore, the availability of CRMs or QC samples is of prime importance. Three plasmid-based CRMs whose suitability has been carefully assessed are now available from IRMM (http://www.irmm.jrc.be) for testing of the G20210A mutation (16-18). The plasmids are designed in a way that they can be used for all methods published up to 2006. Based on these plasmid-type RMs, the desired mutations could be introduced using the site-directed mutagenesis technique, allowing the design of test samples with rare genotypes/mutations.

Quality issues in human genetic testing are particularly important because these tests are carried out in principle only once in a lifetime and false results can lead to inadequate treatment or preventive measures. EQA is the key mechanism to assess the performance of diagnostic medical laboratories and the efficiency of their testing methods. Ring trials also serve as educational tools and training for the participants to sustain improvements in the quality of services. Despite these efforts, relatively high error rates for clinical genetic testing of thrombophilic mutations have been reported, which persist at approximately $5 \%$ without any notable improvement in recent years (26-29).

Overall, the results of this interlaboratory comparison showed expected error rates on the wild-type and G20210A mutation samples without additional mutations. This error rate on the detection of SNPs may result in thousands of misclassifications yearly in the participant laboratories, which emphasizes the need for more detailed scrutiny to identify sources of errors and to eliminate weaknesses.

In this study, the vast majority of the false results arose from inadvertent errors by laboratory personnel and not from technical failure of the method as such. The allele-specific PCR assays proved to be less robust than other techniques, however, and the increased error rate of certain LDTs indicates that they, as a group, have to be more carefully validated.

Although the incidence of rare sequence variants interfering with some genotyping techniques is relatively low, the results of samples having such variants can be easily misinterpreted and lead to an even higher error rate. Most of these variants have been discovered because of the unusual melting temperatures observed using hybridization probes with the LightCycler instrument, which is widely used in clinical and research laboratories. Furthermore, all PCR-based methods can be compromised when mutations occur in the primer annealing region, resulting in insufficient amplifica- tion, unusual observations, and eventually inconclusive results.

In previous studies, proficiency testing was carried out on the most common SNPs, and those studies did not challenge the competence of testing laboratories to recognize and correctly report sequence variants adjacent to the mutation of interest. The present exercise revealed that where additional sequence variants resulted in unusual genotyping data and were therefore in principle detectable, only a fraction of the laboratories recognized and adequately reported unexpected SNPs.

Although the G20210A mutation is a wellcharacterized risk factor for venous thromboembolism, the consequences of these adjacent variants on the analysis results have been investigated infrequently. Such rare polymorphisms should be reported as variants of unknown clinical significance clearly distinct from the wild-type or G20210A mutation alleles (33). In proficiency testing, most participants reporting the unusual results as technical issues are unlikely to apply another technique to check for the presence of rare sequence variants, as they would usually do for real patient samples.

Interestingly, most of the participants applying sequencing as routine procedure for the detection of the G20210A mutation did not report the C20209T variant, although it could be detected from their raw data. Other laboratories sequenced the fragment to understand the reason for the unusual melting curves and were able to identify the additional mutation. Although the number of participants using sequencing techniques was relatively low in this ring trial, the results correlate with the findings of the recent EQUALseq studies (methodologic European external quality assurance for DNA sequencing) (34), where single-base changes also were often unnoticed. Moreover, participants who claimed to apply screening methods for previously unknown mutations in monogenic disorders did not recognize the rare variants at a higher rate than laboratories predominantly testing for well-defined SNPs.

These observations underline the pivotal importance of the laboratory personnel skills and suggested that training may be an efficient tool to improve the quality of genotyping services (35).

\section{Conclusion}

This study revealed that DNA analysis for the detection of the prothrombin G20210A mutation is reliable in a majority of laboratories. However, a fraction of the participants were not able to recognize and adequately report the unusual genotyping results caused by rare SNPs. The majority of failures could be avoided by improved training and careful selection and validation of the methods applied. 
Author Contributions: All authors confirmed they have contributed to the intellectual content of this paper and have met the following 3 requirements: (a) significant contributions to the conception and design, acquisition of data, or analysis and interpretation of data; (b) drafting or revising the article for intellectual content; and (c) final approval of the published article.

Authors' Disclosures of Potential Conflicts of Interest: No authors declared any potential conflicts of interest.
Role of Sponsor: The funding organizations played no role in the design of study, choice of enrolled patients, review and interpretation of data, or preparation or approval of manuscript.

Acknowledgments: We thank Prof. Hans Reinauer and Ralf Flörke (Instand e.V., Düsseldorf, Germany), Prof. Michael Neumaier and Rolf Kruse (DGKL-Referenzinstitut für Bioanalytik, Bonn, Germany), and Erika Sárkány (QualiCont Kht., Szeged, Hungary) for their support and contribution to the ring trial. We gratefully acknowledge the participation of all of the laboratories.

\section{References}

1. Teupser D, Rupprecht W, Lohse P, Thiery J. Fluorescence-based detection of the CETP TaqIB polymorphism: false positives with the TaqManbased exonuclease assay attributable to a previously unknown gene variant. Clin Chem 2001;47: 852-7.

2. Lay MJ, Wittwer CT. Real-time fluorescence genotyping of factor V Leiden during rapid-cycle PCR. Clin Chem 1997;43:2262-7.

3. Jeffrey GP, Chakrabarti S, Hegele RA, Adams PC. Polymorphism in intron 4 of HFE may cause overestimation of C282Y homozygote prevalence in haemochromatosis. Nat Genet 1999;22:325-6.

4. Lyon E. Discovering rare variants by use of melting temperature shifts seen in melting curve analysis [Editorial]. Clin Chem 2005;51:1331-2.

5. Graham R, Liew M, Meadows C, Lyon E, Wittwer CT. Distinguishing different DNA heterozygotes by high-resolution melting. Clin Chem 2005;51: 1295-8.

6. Mahadevan MS, Benson PV. Factor V null mutation affecting the Roche LightCycler factor V Leiden assay. Clin Chem 2005;51:1533-5.

7. Liew M, Pryor R, Palais R, Meadows C, Erali M, Lyon $E$, et al. Genotyping of single nucleotide polymorphisms by high-resolution melting of small amplicons. Clin Chem 2004;50:1156-64.

8. Lyon E, Millson A, Phan T, Wittwer CT. Detection and identification of base alterations within the region of factor $\mathrm{V}$ Leiden by fluorescent melting curves. Mol Diagn 1998;3:203-10.

9. Phillips $M$, Meadows CA, Huang MY, Millson A, Lyon E. Simultaneous detection of $\mathrm{C} 282 \mathrm{Y}$ and H63D hemochromatosis mutations by dual-color probes. Mol Diagn 2000;5:107-16.

10. Tag CG, Gressner AM, Weiskirchen R. An unusual melting curve profile in LightCycler multiplex genotyping of the hemochromatosis H63D/C282Y gene mutations. Clin Biochem 2001;34:511-5.

11. Warshawsky I, Hren C, Sercia L, Shadrach B,

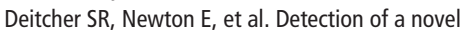
point mutation of the prothrombin gene at position 20209. Diagn Mol Pathol 2002;11:152-6.

12. Wylenzek M, Geisen $C$, Stapenhorst $L$, Wielckens $\mathrm{K}$, Klingler KR. A novel point mutation in the $3^{\prime}$ region of the prothrombin gene at position 20221 in a Lebanese/Syrian family [Letter]. Thromb Haemost 2001;85:943-4.

13. Schrijver I, Lenzi $T J$, Jones $C D$, Lay MJ, Druzin $M L$, Zehnder JL. Prothrombin gene variants in nonCaucasians with fetal loss and intrauterine growth retardation. J Mol Diagn 2003;5:250-3.

14. Tag CG, Schifflers M-C, Mohnen M, Gressner AM, Weiskirchen R. Atypical melting curve resulting from genetic variation in the $3^{\prime}$-untranslated region at position 20218 in the prothrombin gene analyzed with the LightCycler factor II (prothrombin) G20210A assay [Letter]. Clin Chem 2005;51: 1560-1.

15. Klein $C L$, Márki-Zay J, Corbisier $P$, Gancberg $D$, Cooper S, Gemmati D, et al. Reference materials (RMs) for analysis of the human factor II (prothrombin) gene G20210A mutation. Clin Chem Lab Med 2005;43:862-8.

16. Gancberg D, Márki-Zay J, Corbisier P, Klein C, Schimmel H, Emons H. Certification of a reference material consisting of purified plasmid DNA containing a fragment from the human prothrombin gene (wildtype): certified reference material IRMM/IFCC-490. Geel (Belgium): European Commission, Directorate-General Joint Research Centre, Institute for Reference Materials and Measurements; 2006. 35 p. Report EUR 22169 EN.

17. Gancberg D, Márki-Zay J, Corbisier P, Klein C, Schimmel H, Emons H. Certification of a reference material consisting of purified plasmid DNA containing a fragment from the human prothrombin gene (G20210A mutant): certified reference material IRMM/IFCC-491. Geel (Belgium): European Commission, Directorate-General Joint Research Centre, Institute for Reference Materials and Measurements; 2006. 35 p. Report EUR 22170 EN.

18. Gancberg D, Márki-Zay J, Corbisier P, Klein C, Schimmel H, Emons H. Certification of a reference material consisting of purified plasmid DNA containing a fragment from the human prothrombin gene (heterozygous G20210 wildtype/G20210A mutant): certified reference material IRMM/IFCC492. Geel (Belgium): European Commission, Directorate-General Joint Research Centre, Institute for Reference Materials and Measurements; 2006. 33 p. Report EUR 22167 EN.

19. Gancberg D, Corbisier P, Meeus N, Marki-Zay J, Mannhalter C, Schimmel H. Certification of reference materials for detection of the human prothrombin gene G20210A sequence variant. Clin Chem Lab Med 2008;46:463-9.

20. Poort SR, Rosendaal FR, Reitsma PH, Bertina RM. A common genetic variation in the $3^{\prime}$ untranslated region of the prothrombin gene is associated with elevated plasma prothrombin levels and an increase in venous thrombosis. Blood 1996;88:3698-703.

21. McGlennen RC, Key NS. Clinical and laboratory management of the prothrombin G20210A mutation. Arch Pathol Lab Med 2002;126:1319-25.

22. Wylenzek C, Trubenbach J, Gohl P, Wildhardt G, Alkins $S$, Fausett $M B$, et al. Mutation screening for the prothrombin variant G20210A by melting point analysis with the LightCycler system: atyp- ical results, detection of the variant C20209T and possible clinical implications. Clin Lab Haematol 2005:27:343-6.

23. Clench $T$, Standen GR, Ryan E, Chilcott JL, Mumford $A D$. Rapid detection of the prothrombin C20209T transition by LightCycler analysis. Thromb Haemost 2005;94:1114-5.

24. van der Putten HH, Spaargaren-van Riel CC, Bertina RM, Vos HL. Functional analysis of two prothrombin 3 '-untranslated region variants: the C20209T variant, mainly found among AfricanAmericans, and the C20209A variant. J Thromb Haemost 2006;4:2285-7.

25. Meadows CA, Warner D, Page S, Lyon E. Detection of novel mutation using fluorescent hybridization probes and melting temperature analysis [Abstract]. J Mol Diagn 2001;3:195.

26. Jennings I, Kitchen $S$, Woods TA, Preston FE. Multilaboratory testing in thrombophilia through the United Kingdom National External Quality Assessment Scheme (Blood Coagulation) Quality Assurance Program. Semin Thromb Hemost 2005; 31:66-72.

27. Tripodi A, Chantarangkul V, Menegatti M, Tagliabue L, Peyvandi F. Performance of clinical laboratories for DNA analyses to detect thrombophilia mutations. Clin Chem 2005;51:1310-1.

28. Neumaier M, Braun A, Gessner R, Funke H. Experiences with external quality assessment (EQA) in molecular diagnostics in clinical laboratories in Germany. Working Group of the German Societies for Clinical Chemistry (DGKC) and Laboratory Medicine. Clin Chem Lab Med 2000:38:161-3.

29. Tripodi A, Peyvandi F, Chantarangkul V, Menegatti M, Mannucci PM. Relatively poor performance of clinical laboratories for DNA analyses in the detection of two thrombophilic mutations: a cause for concern. Thromb Haemost 2002;88: 690-1.

30. Danneberg J, Abbes AP, Bruggeman BJ, Engel H, Gerrits J, Martens A. Reliable genotyping of the G-20210-A mutation of coagulation factor II (prothrombin). Clin Chem 1998;44:349-51.

31. Spector EB, Grody WW, Matteson CJ, Palomaki GE, Bellissimo DB, Wolff DJ, et al. Technical standards and guidelines: venous thromboembolism (factor V Leiden and prothrombin 20210G $>$ A testing): a disease-specific supplement to the standards and guidelines for clinical genetics laboratories. Genet Med 2005;7:444-53.

32. Chen B, O'Connell CD, Boone DJ, Amos JA, Beck JC, Chan MM, et al. Developing a sustainable process to provide quality control materials for genetic testing. Genet Med 2005;7:534-49.

33. Richards CS, Bale $S$, Bellissimo DB, Das $S$, Grody WW, Hegde MR, et al., Molecular Subcommittee 
Proficiency Testing Study on Rare Sequence Variants

of the ACMG Laboratory Quality Assurance Committee. ACMG recommendations for standards for interpretation and reporting of sequence variations: revisions 2007. Genet Med 2008;10: 294-300.
34. Ahmad-Nejad $P$, Dorn-Beineke $A$, Pfeiffer $U$, Brade J, Geilenkeuser WJ, Ramsden S, et al. Methodologic European external quality assurance for DNA sequencing: the EQUALseq program. Clin Chem 2006;52:716-27.
35. Dorn-Beineke A, Ahmad-Nejad P, Pfeiffer U, Ramsden S, Pazzagli M, Neumaier M. Improvement of technical and analytical performance in DNA sequencing by external quality assessment-based molecular training. Clin Chem 2006;52:2072-8. 